\title{
Impact of iron-deficiency upon behaviour and protein expression in the male mouse with implications for Restless Legs Syndrome
}

\author{
Doctoral Thesis
}

\author{
In fulfillment of the requirements \\ for the degree "Doctor of Philosophy (Ph.D.)" \\ in Neuroscience \\ Georg-August University Göttingen, \\ Faculty of Biology
}

submitted by

\author{
Pascal. H. Dowling \\ BScPharm (Hons), MPhil \\ From the United Kingdom \\ Place of birth Muscat, Oman
}

Göttingen, March 2009 
D 7

Referent: Prof. Dr. Michael Kessel

Korreferent: Prof. Dr. med. Walter Paulus

Tag der mündlichen Prüfung: 31.03.2009 


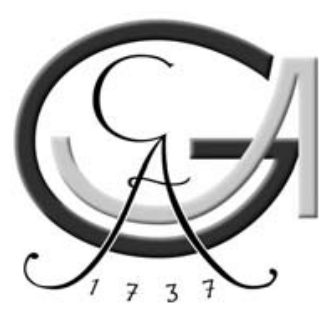

This research commenced in August 2006 and was completed in March 2009. Research was performed at the Department of Clinical Neurophysiology, University Medical Centre Göttingen, Germany. Department director is Professor. Dr. med. Walter Paulus. 
This thesis is dedicated to my parents for all the advice and support they have given me over the years 


\section{DECLARATION}

This work has not previously been accepted in substance for any degree and is not being concurrently submitted in candidature for any degree.

Signed

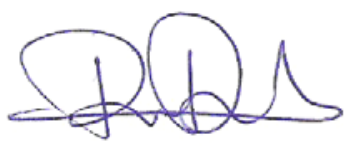

Date

(candidate)

\section{STATEMENT}

This thesis is the result of my own investigations, except where otherwise stated. Other sources are acknowledged by footnotes giving explicit references. A bibliography is appended.

Signed

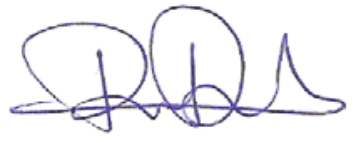

Date

(candidate)

16.03.2009 


\section{Contents}

Acknowledgements I

Summary II

Figures IV

Tables $\quad$ V

$\begin{array}{ll}\text { Abbreviations } & \text { VI }\end{array}$

\section{Chapter 1. Introduction 1}

1.1. $\quad$ Restless Legs Syndrome 1

1.1.1. History of RLS 1

1.1.2. Epidemiology of RLS 1

1.1.3. Symptoms of RLS 2

1.1.4. Pathophysiology of RLS 3

1.1.5. Involvement of iron 4

1.1.5.1. Iron in RLS 4

1.2. Importance of iron 4

1.2.1. The consequences of inducing iron-deficiency 5

1.2.1.1. $\quad$ Animal behaviour and physiology 5

1.2.1.2. Development 5

1.2.2. Importance of iron in homeostasis of the dopaminergic system 6

1.2.2.1. Tyrosine hydroxylase $\quad 6$

1.2.2.2. Dopamine transporter and receptors $\quad 6$

1.2.3. Involvement of other neurotransmitters 7

1.2.3.1. Choline acetyltransferase $\quad 7$

1.2.4. Function of myelin proteins during iron-deficiency 8

1.2.4.1. Implications of the sirtuin 2 protein in myelin 9

1.3. Central nervous involvement in RLS 9

1.3.1. Supraspinal regions $\quad 10$

1.3.2. Dorsal horn region of the spinal cord 11

1.3.3. Ventral horn region of the spinal cord 13

1.4. Animal models of RLS $\quad 15$

1.4.1. Current animal models of RLS 15

1.4.2. Approach in designing an animal model of RLS $\quad 18$

1.4.2.1. The sensory component 18

1.4.2.2. The motor component and identifying the circadian onset 19

1.4.3. Direction of future animal models 20

1.4.4. Importance for research 21

1.5. Aims and objectives of this thesis 21 
2.1. Controlling iron consumption in the mouse's environment 23

2.2. Evaluating the effect of ID diet upon the general physiology 23

2.3. Assessing sensory behavioural differences 26

2.3.1. The hot-plate test 26

2.3.1.1. $\quad$ Iron-deficiency reduces hot-plate reaction time in WT mice 26

2.3.1.2. $\quad$ Reduced hot-plate reaction time in $D 3^{-/-}$mice 26

2.3.2. The formalin-test 27

2.3.2.1. Iron-deficiency elevates inflammatory pain response in WT mice 27

2.3.2.2. Iron-deficiency synergistically elevates the inflammatory

2.3.3. Heightened central cellular activity detected by ipsilateral

2.4. Assessing motor behavioural differences 32

2.4.1. The voluntary wheel-running test 32

2.4.1.1. Wheel-running performance in WT mice $\quad 32$

2.4.1.2. Iron-deficiency increases wheel-running activity in WT mice 33

2.4.1.3. Wheel-running performance in D $3^{-/-}$mice 37

2.4.1.4. Iron-deficiency increases wheel-running activity in D3 $3^{-/-}$mice 38

2.4.1.5. Differences in wheel-running activity between WT and D $3^{-/}$mice 42

2.4.2. Increased ChAT immunoreactivity upon motoneurons at spinal T13-L2 48

2.5. Assessing protein expression differences in the CNS $\quad 51$

2.5.1. 2-D DIGE Gel electrophoresis of mouse midbrain $\quad 51$

2.5.1.1. $\quad$ Altered protein expression in ID WT mice at 15 weeks diet duration $\quad 51$

2.5.2. Identification of 2-D DIGE protein samples from WT mouse midbrain by using MALDI-TOF-MS $\quad 53$

2.5.2.1. MASCOT score analysis $\quad 53$

$\begin{array}{lll}\text { 2.5.2.2. } & \text { Protein identification } & 53\end{array}$

2.5.3. Quantitative Western blotting 55

2.5.3.1. $\quad$ Mouse midbrain $\quad 55$

2.5.3.2. $\quad$ Mouse spinal cord $\quad 57$

2.5.4. Quantitative assessment of SIRT2 v2 variant in midbrain tissue from

2-D IEF/SDS-PAGE blots 58

\section{Chapter 3. Discussion}

3.1. Evaluating the effects an ID diet has upon the general physiology of WT mice $\quad \mathbf{6 0}$

3.1.1. Influence of ID diet upon weight and muscle strength in WT mice 61

3.1.2. Influence of ID diet upon haematology in WT mice 61

3.1.2.1. ID diet reduces the plasma-iron concentration 61

3.1.2.2. ID diet alters haemoglobin and haematocrit 62 
3.2. The effect of iron-deficiency upon sensory behaviour in WT and $\mathrm{D3}^{-/-}$mice

3.2.1. ID WT mice show elevated acute and inflammatory pain responses assessed by the hot-plate and formalin tests

3.2.2. Increase in acute pain response in $\mathrm{D}^{-/-}$mice and a synergistic increase in prolonged inflammatory pain induced by iron-deficiency $\quad 65$

3.2.2.1. Involvement of dopamine and possibly glutamate receptors in enhancing acute and persistent pain responses in $\mathrm{D}^{--}$mice.

3.3. The effect of iron-deficiency upon the pattern of circadian motor activity

3.3.1. Iron-deficiency elevates voluntary wheel-running activity in WT and $\mathrm{D}^{-/-}$mice

3.3.1.1. Involvement of iron-deficiency and the dopaminergic system 70

3.3.2. Chronic administration of ID diet has little suppressive effect upon wheel-running performance in $\mathrm{WT}$ and $\mathrm{D} 3^{-/-}$mice

3.3.3. Iron-deficiency increases innervation of ChAT-IR terminals upon ventral motoneurons

3.4. Influence iron-deficiency has upon protein expression in the CNS $\quad 74$

3.4.1. Identified proteins $\quad 74$

$\begin{array}{ll}\text { 3.4.2. } & \text { SIRT2 and myelin markers } \\ 3.4 .3 . & 76\end{array}$

$\begin{array}{lll}\text { 3.4.3. } & \text { Transporter and enzymes } & 76\end{array}$

$\begin{array}{lll}\text { 3.5. } & \text { Conclusions } & 77\end{array}$

$\begin{array}{lll}\text { 3.6. } & \text { Current and future research } & 78\end{array}$

Chapter 4. Materials and Methods $\quad 80$

$\begin{array}{llr}\text { 4.1. } & \text { Animals } & 80\end{array}$

4.1.1. Breeding 80

4.1.2. Housing conditions $\quad 81$

$\begin{array}{lll}\text { 4.1.3. Diets } & 81\end{array}$

$\begin{array}{lll}\text { 4.2. } & \text { Assessing sources for iron-content } & 81\end{array}$

$\begin{array}{llr}\text { 4.3. } & \text { Behavioural experiments } & 82\end{array}$

$\begin{array}{lll}\text { 4.3.1. } & \text { Forelimb grip-strength test } & 82\end{array}$

4.3.2. Hot-Plate test $\quad 82$

4.3.3. Formalin-test $\quad 83$

4.3.4. Voluntary wheel-running experiments $\quad 84$

4.4. $\quad$ Programming for voluntary wheel-running experiments $\quad 84$

4.5. Anaesthesia and blood extraction $\quad 85$

4.5.1. Anaesthesia 85

4.5.2. $\quad$ Extracting blood from the mouse $\quad 85$

4.5.3. $\quad$ Preparing blood for analysis 86

4.5.3.1. $\quad$ Diluting plasma samples prior to analysis 86

4.5.4. Plasma-iron $\quad 86$

$\begin{array}{llr}\text { 4.5.5. } & \text { Haematocrit } & 87\end{array}$

$\begin{array}{lll}\text { 4.5.6. } & \text { Haemoglobin } & 87\end{array}$ 
4.6.

Tissue extraction and immunohistochemistry

4.6.1.

Tissue preparation, sectioning and storage

4.6.2. Immunohistochemistry for c-fos at L4-L6

4.7.

4.7.1.

Tissue and protein preparation 93

4.7.2.

Stereotaxic positions of the midbrain

4.7.3.

Tissue preparation 93

4.7.4.

Tissue homogenisation 94

4.7.4.1. Bicinchoninic acid protein colourimetric assay for 2-D Gel electrophoresis

4.8.

1-D/2-D gel electrophoresis 97

4.8.1. 1-D DIGE gel electrophoresis

4.10.

4.13. 


\section{Acknowledgements}

I particularly wish to thank my mentor Professor Walter Paulus for his time, dedication, guidance and inspiring ideas he has given throughout my research in Göttingen. I would also like to thank Dr David Liebetanz for his helpful supervision, time and patience throughout my PhD. In addition, I am grateful to Professor Michael Kessel from the Max Planck Institute for Biophysical Chemistry in Göttingen, for his advice, patience and constructive comments concerning my thesis. Moreover, I would like to thank Dr Michael Nitsche from the University Medical Centre in Göttingen for his guidance and advice concerning the statistical methods employed in this thesis and to Dr Florian Klinker for designing the wheel-running software. Furthermore, I wish to thank Professor Christine Stadelmann and Dr Doron Merkler from the the University Medical Centre in Göttingen, department of Neuropathology for their helpful comments regarding the experimental procedures of immunohistochemistry. Additionally, I am grateful to Professor Markus Otto and Dr Stefan Lehnert from the Centre for Clinical Research in Ulm, for their assistance and guidance in the techniques of 2-D Gel electrophoresis and 2-D IEF/SDS-PAGE. Also I wish to thank Dr Olaf Jahn from the Proteomics Group of the Max Planck Institute for Experimental Medicine in Göttingen for performing MALDI-TOF-MS and providing useful advice concerning the protein work. In addition, I thank Dr Hauke Werner from the department of Neurogenetics of the Max Planck Institute for Experimental Medicine for his constructive advice during Western blotting and related protein research. I would also like to thank Dr Dieter Scheller from UCB Biopharma for his time in reading my thesis and giving useful advice. Last but not least, I wish to thank Professor Edgar Brunner from the department of medical statistics for his useful comments and ongoing statistical help regarding the protein data.

I wish to achknowledge Marion Kurze for her continuous help with all the paper work and together with Christine Crozier for giving much needed advice to me when first arriving in Göttingen.

Finally, I want to thank all my family particularly my parents to whom this thesis is dedicated to. Also, I would like thank my sister Aileen for providing her never ending experience and advice concerning my $\mathrm{PhD}$ and to my other sister Sara for her much needed support and strong words. Furthermore, I wish to express my gratitude to my brother Patrick for his continuous encouragement. Lastly, I would like to acknowledge Katharina Tretter for always supporting me and showing patience and understanding. 


\section{Summary}

Restless legs syndrome (RLS) comprises of both sensory and motor symptoms, observed as an increased pain and periodic jerking movement of the legs. There are two forms of RLS; one being the genetic (primary) form whereby 4 gene variants have been identified, the second form is non-genetically characterised (secondary), where iron-deficiency is of major importance. Dopaminergic therapy has a central role in treating both forms of RLS suggesting an important function of the dopaminergic system, which in turn is known to be dysregulated by iron-deficiency via iron dependence of tyrosine hydroxylase and the dopamine transporter. The aim of this thesis was to devise an animal model of secondary RLS and to correlate whether iron-deficiency is the inducing factor of the behavioural sensory and motor symptoms in-vivo.

A nutritional deprivation of iron in C57B1/6 wildtype (WT) and dopamine receptor 3 null (D3${ }^{\prime}$ ) male mice was induced by an iron-deficient (ID) diet starting from post-natal day 28 (P28) and continuing for 1, 4, 8, 15 or 30 weeks duration. By using the hot-plate and formalin tests after 1, 4 and/or 15 weeks of ID diet, acute and persistent sensory responses were tested upon activation of primarily afferent $\mathrm{A} \delta$ - and C-fibres, respectively, where these fibres have been shown in clinical studies to be altered in both primary and secondary RLS patients. c-Fos immunoreactive (c-Fos-IR) cells at the ipsilateral horn were quantified accordingly.

A voluntary wheel-running system measured changes in circadian running activity after 4 and 15 weeks of ID diet. Protein analysis was performed on the entire midbrain region from the 15 week ID and control WT mouse groups. Screening for proteins that show changes during iron-deficiency was performed by two dimension fluorescence difference in gel electrophoresis (2-D DIGE). Afterwards, proteins were identified using matrix-assisted laser desorption/ionisation time-of-flight mass spectrometry (MALDI-TOF-MS) followed by quantitative Western blotting and 2-D isoelectic focussing followed by SDS-PAGE (2-D IEF/SDS-PAGE).

The main results are:

1) The acute pain response was elevated in ID WT mice after 4 and 15 weeks. After 15 weeks of diet, $\mathrm{D}^{-/-}$mice exhibit an increased acute pain response compared to WT mice. Irondeficiency did not further heighten the acute pain response in $\mathrm{D} 3^{-/-}$mice.

2) The persistent pain response was increased in ID WT mice after all durations of ID diet (1, 4 and/or 15 weeks). After 15 weeks of diet, $\mathrm{D}^{-/-}$mice showed an elevated pain response which was further increased by iron-deficiency. 
3) After 15 weeks of ID diet, an increased running activity (number of runs and activity time) was shown in ID WT mice specifically 2 hours before the end of the active dark phase (06:00hr-07:00hr). ID D3 ${ }^{-/-}$mice showed a heightened running activity at an earlier time period (03:00hr-04:00hr).

4) In total 14 protein targets were dysregulated, 5 of which were identified by MALDI-TOFMS. One of these proteins was sirtuin 2 (SIRT2), where its expression was elevated by 20\% in ID WT mice. Quantitative Western blotting and 2-D IEF/SDS-PAGE identified non-significant changes, however all techniques confirm a similar increase in SIRT2 protein expression at the short SIRT2 variant 2 (v2) localised predominantly in myelin. Isoform 2 of SIRT v2 which has an unknown function shows this increase. Proteolipid protein/DM20 (PLP/DM20) and dopamine transporter (DAT) show similar trends to that previously described in other ID rodent studies.

The work reported in this thesis has identified iron-deficiency as a factor in increasing both the sensory perception and modifying the circadian onset of increased motor activity. The pain tests show increased acute and persistent inflammatory pain responses, in addition to an elevated motor activity and activity time prior to the sleep phase in mice, corresponding to a period showing increased RLS complaints in humans. In the absence of D3 receptors, irondeficiency has a synergistic role in shifting the onset of heightened activity to an earlier onset by at least 2 hours, which is characteristic of augmentation described in human RLS under dopaminergic therapy. These behavioural tests have proved effective in describing both sensory and motor changes which are comparable to those in RLS patients. Moreover, this is the first animal model which shows the closest match to RLS symptoms. In addition to the behavioural studies, the identification of SIRT2 in relation to iron-deficiency by using MALDI-TOF-MS is a novel finding.

To date there is no animal model based on the genetic variants identified. Therefore, investigating changes caused by iron-deficiency in the mouse compared to the rat due to its fully identified genome, offers the most promising approach. Findings from these mouse studies will prove important in the future by contributing to the design of a genetic mouse model of RLS. 


\section{Figures}

Figure 1.1. Monoaminergic pathways originating from supraspinal regions and innervating the dorsal horn

Figure 1.2. Overview of neurotransmitters, modulators and receptors involved in relaying nociceptive information at the dorsal horn

Figure 1.3. Distribution of interneuron groups at T13-L2 spinal segments $\quad \mathbf{1 4}$

Figure 2.1. Effect of ID diet upon general physiology $\quad \mathbf{2 5}$

Figure 2.2. Effect of ID diet upon hot-plate reaction time $\quad 27$

Figure 2.3. The effect of ID diet on formalin-induced pain $\quad 28$

Figure 2.4. The effect of different durations of ID diet in WT mice upon formalin-induced c-Fos expression

Figure 2.5. Influence of ID diet and duration upon voluntary wheel-running performance in WT mice

Figure 2.6. ID diet alters the activity shown by number of runs during the late phase of the active cycle in WT mice

Figure 2.7. ID diet alters the activity time of wheel-running during the late phase of the active cycle in WT mice

Figure 2.8. Influence of ID diet and duration upon voluntary wheel-running performance in $\mathrm{D} 3^{-/-}$mice

Figure 2.9. ID diet alters the activity shown by number of runs during the dark phase in $\mathrm{D}^{-/-}$mice

Figure 2.10. ID diet alters the activity time of wheel-running during the dark phase in $\mathrm{D} 3^{-/-}$mice

Figure 2.11. Double plotted actograms of $\mathrm{WT}$ and $\mathrm{D} 3^{-/-}$mice

Figure 2.12. Combined plots showing differences in activity by number of runs between $\mathrm{WT}$ and $\mathrm{D} 3^{-/-}$mice

Figure 2.13. Combined plots showing differences in wheel-running activity time between $\mathrm{WT}$ and $\mathrm{D} 3^{-/-}$mice

Figure 2.14. The effect of ID diet on ChAT-IR expression upon motoneurons at the $\mathrm{CPG}$ region in WT mice

Figure 2.15. 2-D DIGE gel showing protein expression differences of the midbrain from control WT and ID WT mice at 15 weeks diet duration

Figure 2.16. Quantitative Western blotting of control WT and ID WT midbrain for SIRT2, myelin and candidate markers

Figure 2.17. Quantitative Western blotting of control WT and ID WT spinal cord for SIRT2, myelin and candidate markers

Figure 2.18. 2-D IEF/SDS-PAGE blots of control and ID WT midbrain for SIRT2 v1 and v2 variants

Figure 4.1. Dissected midbrain region for protein identification 


\section{Tables}

Table 1.1. Existing animal models relating to RLS

Table 2.1. Iron concentration of different components in the mouse's environment

Table 2.2. Results of the ANOVAs 31

Table 2.3. Results of the ANOVAs - Voluntary wheel-running performance $\quad 46$

Table 2.4. Results of the ANOVAs - Voluntary wheel-running activity_ 47

Table 2.5. Results of the ANOVAs $\quad \mathbf{5 0}$

Table 2.6. Differences in protein expression between control WT and ID WT mice $\quad \mathbf{5 2}$

Table 2.7. MALDI-TOF-MS identified proteins $\quad 54$ 


\section{Abbreviations}

1-D First-dimension

2-D

Second-dimension

2-D DIGE

Two dimension fluorescence difference in gel electrophoresis

2-D IEF/SDS-PAGE Two dimension isoelectric focussing/sodium dodecyl sulphate polyacrylamide gel electrophoresis

5-HT 6-OHDA

A

A11

Acetyl-CoA

$\mathrm{ACh}$

aCINs

adCINs

AMPA

ANS

APS

ATP

A $\delta$

BCA

BSA

BVA

c-Fos-IR

ChAT

ChAT-IR

CINs

CNS

CPG

Cy2

Cy3

Cy5

D1-D5

D3 ${ }^{-/-}$

DA

DAB

DAPI

DAPI

DAT

dCINs

$\mathrm{ddH}_{2} \mathrm{O}$

Distance/ $\mathrm{N}_{\text {run }}$

DMF

EDTA

EEG

ELISA

EMG

EPSP

FCS

$\mathrm{Fe}^{2+}$

FITC

$\mathrm{g} / \mathrm{dL}$

GABA

GAPDH

GDNF
Serotonin

6-hydroxydopamine

Ampere

Diencephalic-spinal A11 nuclei

Acetyl-coenzyme A

Acetylcholine

Ascending commissural interneurons

Ascending/descending commissural interneurons

$\alpha$-amino-3-hydroxyl-5-methyl-4-isoxazole-propionate

Autonomic nervous system

Ammonium persulfate

adenosine 5'-triphosphate

A delta fibre

Bicinchoninic acid

Bovine serum albumin

Biological variation analysis

c-Fos immunoreactive

Choline acetyltransferase

Choline acetyltransferase immunoreactive

Commissural interneurons

Central nervous system

Central pattern generator

Indocarbocyanine 2

Indocarbocyanine 3

Indocarbocyanine 5

Dopamine receptors 1-5

Dopamine receptor 3 homozygous knock-out

Dopamine

3,3'-Diaminobenzidine tetra-hydrochloride

4',6-diamidino-2-phenylindole

4',6-diamidino-2-phenylindole

Dopamine transporter

Descending commissural interneurons

Double distilled water

Distance per run (metres/run)

Dimethylformamide

Ethylenediaminetetraacetic acid

Electroencephalographic

Enzyme-linked immunosorbent assay

Electromyographic

Excitatory postsynaptic potential

Fetal calf serum

Ferrous iron

Fluorescein isothiocyanate

Grams per deciliter

Gamma-aminobutyric acid

Glyceraldehyde 3-phosphate dehydrogenase

glial cell line-derived neurotrophic factor 


\begin{tabular}{|c|c|}
\hline GFAP & Glial fibrillary acidic protein \\
\hline $\mathrm{HCl}$ & Hydrochloric acid \\
\hline $\mathrm{hr}$ & Hour \\
\hline HRP & Horseradish peroxidase \\
\hline IAA & Iodoacetamide \\
\hline Ia-INs & Ia interneurons \\
\hline ID & Iron-deficient \\
\hline IEF & Isolectric focussing \\
\hline IML & Intermediolateral nucleus \\
\hline $\mathrm{k} / \mathrm{o}$ & Knock-out \\
\hline $\mathrm{kDa}$ & Kilodaltons \\
\hline KVh & Kilovolts per hour \\
\hline L1-L2 & Lumbar segments $1-2$ \\
\hline L-DOPA & Levodopa (3,4-dihydroxy-L-phenylalanine) \\
\hline LTP & Long term potentiation \\
\hline $\mathrm{m}$ & metres \\
\hline $\mathrm{m} / \mathrm{run}$ & metres/run \\
\hline $\mathrm{m} / \mathrm{s}$ & metres/second \\
\hline M3 & Muscarinic receptor type 3 \\
\hline MALDI-TOF-MS & $\begin{array}{l}\text { Matrix-assisted laser desorption/ionization time-of-flight mass } \\
\text { spectrometry }\end{array}$ \\
\hline $\mathrm{MeOH}$ & Methanol \\
\hline MOG & Myelin oligodendrocyte glycoprotein \\
\hline mRNA & Messenger ribonucleic acid \\
\hline MS & Mass spectrometry \\
\hline NAcc & Nucleus accumbens \\
\hline NADH & Nicotinamide adenine dinucleotide \\
\hline NDS & Normal donkey serum \\
\hline $\mathrm{NE}$ & Norepinephrine \\
\hline NET & Norepinephrine transporter \\
\hline NGF & Nerve growth factor \\
\hline NMDA & N-methyl-D-aspartic acid \\
\hline $\mathrm{N}_{\text {run }}$ & Number of runs \\
\hline NSE & Neuron-specific enolase \\
\hline $\mathrm{P}$ & Post-natal \\
\hline $\mathrm{P} 28$ & Post-natal day 28 \\
\hline PAG & Periaqueductal grey \\
\hline PAM & Periodic arm movements \\
\hline PBS & Phosphate-buffered saline \\
\hline PCR & Polymerase chain reaction \\
\hline PFA & Paraformaldehyde \\
\hline $\mathrm{pI}$ & Isoelectric point \\
\hline PLMs & Periodic limb movements \\
\hline PLMS & Periodic limb movements of sleep \\
\hline PLMWA & Periodic limb movements while awake \\
\hline PLP/DM20 & Proteolipid protein/DM20 \\
\hline PMF & Peptide mass fingerprint \\
\hline PVDF & Polyvinylidene difluoride \\
\hline $\mathrm{RBCs}$ & Red blood cells \\
\hline $\mathrm{RCs}$ & Renshaw cells \\
\hline RLS & Restless legs syndrome \\
\hline RVMM & Rostral ventromedial medulla \\
\hline $\mathrm{SCN}$ & Suprachiasmatic nuclei \\
\hline SDS & Sodium dodecyl sulfate \\
\hline SDS-PAGE & Sodium dodecyl sulfate polyacrylamide gel electrophoresis \\
\hline
\end{tabular}




$\begin{array}{ll}\text { SERT } & \text { Serotonin transporter } \\ \text { Sir2p } & \text { Silent information regulator 2 protein } \\ \text { SIRT2 } & \text { Sirtuin } 2 \\ \text { SN } & \text { Substantia nigra } \\ \text { SP } & \text { Substance P } \\ \text { T13 } & \text { Thoracic segment } 13 \\ \text { TBS } & \text { Tris-buffer saline } \\ \text { TBST } & \text { Tris-buffer saline tween } \\ \text { TEMED } & N, N, N^{\prime}, N^{\prime} \text { tetramethylethylenediamine } \\ \text { Tf } & \text { Transferrin } \\ \text { Tf-TfR } & \text { Transferrin-transferrin receptor complex } \\ \text { TH } & \text { Tyrosine hydroxylase } \\ \mathrm{T}_{\text {run }} & \text { Total activity time (minutes) } \\ \text { V } & \text { Volts } \\ \text { v1 } & \text { Variant } 1 \text { of sirtuin 2 } \\ \text { v2 } & \text { Variant } 2 \text { of sirtuin } 2 \\ \text { VAChT } & \text { vesicular acetylcholine transporter } \\ \mathrm{V}_{\text {max }} & \text { Maximum running speed (metres/second) } \\ \text { VMPJ } & \text { Ventral mesopontine junction } \\ \text { v/v } & \text { Volume/volume } \\ \text { W } & \text { Watt } \\ \text { w/v } & \text { Weight/volume } \\ \text { WT } & \text { Wild-type (C57Bl/6 strain) } \\ \lambda & \text { Wavelength } \\ \mu \mathrm{g} & \text { Microgram } \\ \mu 1 & \text { Microlitre } \\ \mu \mathrm{M} / \mathrm{L} & \text { Micromoles per litre } \\ & \end{array}$


Chapter 1

Introduction 


\section{Introduction}

Restless legs syndrome (RLS) is grouped into two categories. Firstly, idiopathic (primary) RLS describes onset of symptoms primarily before 40 years of age and is correlated to a familial history. Comparatively, the second form termed symptomatic (secondary) RLS occurs in later years, between 40-60 years of age and is associated with many other clinical conditions, the most prevalent and important of them to be discussed is iron-deficiency. Both forms of RLS exhibit common symptoms which will be described at a later stage.

This introduction will initially describe the basic clinical features occurring in RLS and will then progress onto introducing the importance of iron and describe the effects irondeficiency has upon animal behaviour, physiology, development and in addition neurotransmitters and myelin. Neuroanatomical structures which are involved or participate in the onset of symptoms will be introduced. In addition, the existing animal models of RLS will be highlighted. Finally, the correct approach for designing a suitable animal model based on clinical findings will be described as will the importance of designing an animal model for investigating secondary RLS in-vivo.

\subsection{Restless Legs Syndrome}

\subsubsection{History of RLS}

Thomas Willis in 1685 initially described symptoms of an unknown disorder that we characterise today as RLS (Willis, 1685). Karl Ekbom in 1944 began to analyse the syndrome in great detail and in 1945 conceived the syndrome's name as "Restless legs Syndrome", a name indicative of the main predisposing symptom (Ekbom, 1944; Ekbom, 1945).

Periodic limb movements (PLMs) are a clinical symptom commonly associated in RLS and is observed as a periodic rhythmic movement of the legs. This symptom was previously known as nocturnal myoclonus (Symonds, 1953), however it was later evaluated in the 1960s by Lugaresi and colleagues (1965) and termed PLMs (Lugaresi, 1965).

\subsubsection{Epidemiology of RLS}

Commonly under diagnosed and often prevailing from middle to later life, RLS is now increasing in commonality with $9-14 \%$ prevalence in Caucasian females and $5.4-9.4 \%$ in Caucasian males, in Europe and North America (Allen et al., 2005; Hogl et al., 2005; Pichler et al., 2008).

Ethnic variation accounts for the vast difference in RLS prevalence between Western countries and that observed in Asian populations. Only a $0.6 \%$ and $0.1 \%$ prevalence was observed in Asian populations aged 55 years or older and 21 years or older, respectively (Tan 
et al., 2001). RLS epidemiology in Indian and Japanese populations were last recorded at $0.8 \%$ and 1.06\%, respectively (Krishnan et al., 2003; Barriere et al., 2005; Mizuno et al., 2005b).

\subsubsection{Symptoms of RLS}

The clinical symptoms of RLS were outlined in an International Restless Legs Syndrome Study Group, which have now become the worldwide accepted criteria for clinical diagnosis of RLS (Allen et al., 2003).

Certain standards published by the Academy of Sleep Medicine list three diagnostic markers: 1) Night time occurrence, 2) unpleasant sensory sensations, and 3) relief of these sensations by movement.

Additional criteria were later implemented, which comprise the standard diagnostic criteria (Allen and Earley, 2001):

1) A sensation of an urge to move the limbs (usually legs).

2) Motor restlessness to reduce sensations.

3) Quiescegenic: onset or worsening of symptoms when at rest.

4) Marked circadian variation in occurrence or severity of symptoms (Walters, 1995).

A fifth supportive set of criteria to the accepted main points above includes, a response to dopaminergic agents and a familial history (Allen et al., 2003).

Symptoms often begin during the evening (after 6pm) or at night time, however, in severe cases the symptoms may prevail earlier, or all day (Walters, 1995; Allen and Earley, 2001; Bonati et al., 2003). Usually the unpleasant urge to move the legs occurs prior to sleep, however an onset of symptoms during sleep may occur causing interruption of sleep due to the involuntary and frequent movement of the legs awaking the individual. This is termed periodic limb movements of sleep (PLMS). When RLS arises early in life the symptoms often begin sporadically featuring a mild discomfort of the legs when at rest. After the age of 50 years, the symptoms often progress to the extent where a daily occurrence of sleep disruption becomes normal (Allen and Earley, 2001).

Shifting of symptoms from evening to an earlier daytime prevalence is most commonly associated with augmentation. The clinical features of augmentation (Allen et al., 2003) include: a) positive feedback between an increase in symptom severity and an increase in medication, thus furthering the prolongation of symptoms. b) Secondly, when symptoms worsen a change in therapy is implemented. c) Earlier daytime symptom onset (2-4 hours), resulting from treatment with certain dopamine agonists. d) Treatment induced shortened 
symptom delay during rest. f) Spreading of symptoms to other body regions (Allen and Earley, 1996; Collado-Seidel et al., 1999).

\subsubsection{Pathophysiology of RLS}

The dopamine rich bilateral dorsomedial hypothalamic nucleus, or more commonly known as the diencephalic-spinal (A11) area serves as the only source of dopamine innervation for the spinal cord (Skagerberg and Lindvall, 1985). These cells have also proved a possible new target for understanding the pathophysiological abnormalities occurring in RLS. The reason for the possible involvement of the dopaminergic A11 region, stems from successful treatment of both primary and secondary RLS using dopamine agonists, inferring dopamine as a dysfunctional mediator of RLS symptoms (Trenkwalder et al., 2005). Next to the A11 neurons and within the hypothalamus are nuclei important in sleep function, therefore supporting the possible role A11 nuclei may play in RLS. Currently, no studies so far have illustrated the changing function of the A11 cell group, partly due to limitations in neuroimaging techniques (Paulus et al., 2007a).

The substantia nigra (SN) is altered in RLS (Godau et al., 2007), however, the exact function of its role in RLS and how it affects the spinal cord remains largely unknown. The involvement of the SN in RLS has been shown by observing RLS in Parkinson's disease patients. RLS has a relatively high prevalence in Parkinson's disease, $20.8 \%$ in one study (Ondo et al., 2002). In addition, RLS symptomatology prevails in Parkinson's disease patients in later stages of life, where they present with low serum ferritin and no correlation with familial RLS (Tan et al., 2002). Furthermore, 15\% of treated Parkinson's disease patients show PLMS, a higher proportion compared to untreated patients. Currently, no evidence suggests an earlier onset of RLS predisposes an individual to Parkinson's disease (Ondo et al., 2002).

Functional magnetic resonance imaging studies have demonstrated that during sensory RLS symptoms there are contralateral and bilateral activations of the thalamus and cerebellum, respectively. In addition, the pons and red nucleus were also shown to be activated during PLM with sensory symptoms, (Bucher et al., 1997). These subcortical generators are involved in RLS symptom aetiology (Barriere et al., 2005). Despite identifying supraspinal structures involved during RLS symptom onset, no gross anatomical differences and deficiencies at dopamine rich brain regions were identified after neuropathological examination between RLS and control brains, for example no alpha-synuclein Lewy cell bodies were observed (Allen and Earley, 2001). However, other changes have been found including a decrease in the endogenous opioids met-enkephalin and beta-endorphin in the thalamus of post-mortem brains from RLS patients (Walters et al., 2009). 


\subsubsection{Involvement of iron}

According to the world health organisation (WHO) 2002 figures, nutritional iron-deficiency is the most common nutrition problem worldwide. Current estimates are up to 2 billion people (Salomon, 2000). Iron-deficiency is known to have many detrimental effects upon the general physiology. This includes impaired thermoregulation, immune response and reduced work capacity (Dobbing, 1990).

\subsubsection{Iron in RLS}

Ekbom first reported that $25 \%$ of patients with severe RLS showed a reduced serum iron level (Ekbom, 1960). Many other studies present varying results, where normal ferritin $(50 \mu \mathrm{g} / \mathrm{l})$, transferrin (Tf) and iron concentrations have also been shown in RLS patients (O'Keeffe et al., 1994; Sun et al., 1998; Allen and Earley, 2000). Confirmation from several RLS clinical studies as described below, identify a reduced ferritin and iron level in serum (O'Keeffe et al., 1993; O'Keeffe et al., 1994; Sun et al., 1998), where often an inverse relationship exists between the level of ferritin and severity of RLS symptoms (O'Keeffe et al., 1994; Sun et al., 1998). One study showed a correlation between low iron and RLS during iron-deficiency anaemia and pregnancy (Mizuno et al., 2005a). Additional studies show low ferritin and iron levels in akathisic patients who present similar symptoms to RLS (Gold and Lenox, 1995; Sachdev, 1995). Other symptoms in RLS, for example sensory neuropathy have not been associated with a reduced iron status (Salvi et al., 1990; Ondo and Jankovic, 1996; Allen and Earley, 2001). The participating role of iron is considered secondary or symptomatic in RLS (Berger et al., 2002).

The localisation of iron implicates it has an important role in RLS symptomatology. A reason for this, is that certain brain regions associated with motor function display the highest iron concentrations (Koeppen, 1995). The striatum, red nucleus, part of the hippocampus and deep cerebellar nucleus show a high density of iron, particularly the association of iron bound to neuromelanin in SN dopaminergic neurons. Moreover, during age iron accumulates in the brain, particularly in the SN (Barriere et al., 2005). The involvement of other central structures will be introduced later in section 1.3.

\subsection{Importance of iron}

Iron is an important metal ion which when dysregulated may produce changes in several behavioural and cellular processes, muscle function (Dallman, 1986) and neurotransmitter synthesis (Rang, 1999; Nestler, 2001). This section is concerned with illustrating the effects a 
reduced physiological iron content has upon mammalian physiology and development, the dopaminergic system and finally neurotransmitters and myelin.

Iron-deficiency results in altered brain function through hypomyelination, delayed neurodevelopment and dysfunction of the dopaminergic system (Yu et al., 1986; Beard et al., 2003b). Subsequently, iron-deficiency in humans and animal models changes behaviour and cognition (Bourque et al., 2008). In addition, other changes are observed for example an altered neurotransmitter function and a reduced locomotor activity and learning, which are suppressed as a result of reduced brain iron content (Youdim et al., 1981; Yehuda et al., 1986).

\subsubsection{The consequences of inducing iron-deficiency}

\subsubsection{Animal behaviour and physiology}

Commonly, the effects of an iron-deficient (ID) diet causes a reduced body weight in rodents (Beard et al., 1995; Kwik-Uribe et al., 1999), changes in haemoglobin, haematocrit (Beard et al., 1995), serum iron (Qu et al., 2007) and brain/spinal iron concentrations (Qu et al., 2007; Zhao et al., 2007). Behavioural abnormalities induced by iron-deficiency in rodents have been previously described as: increased anxiety, reduced exploratory behaviour, inconsistent grooming pattern and delayed forelimb placing reflex (Ward et al., 2007). Other behavioural differences include changes in learning/motor performance and diminished grip strength (Kwik-Uribe et al., 1999; Kwik-Uribe et al., 2000b; Garcia et al., 2007). These animal behavioural observations are the result of inducing iron-deficiency during embryonic development, lactation and postweaning periods.

\subsubsection{Development}

Differences in iron distribution are observed when inducing iron-deficiency either neonatally or at postweaning stages during rodent development (Beard and Connor, 2003). During lactation (Pinero et al., 2000) and weaning (Erikson et al., 2000) a greater reduction in brain iron $(30-50 \%)$ is observed when compared to an early-age onset of iron-deficiency. The level of brain iron concentration after inducing iron-deficiency by an ID diet, depends upon the start point of dietary intervention during development and the brain region in question. For example, how the nucleus accumbens (NAcc) compared to the striatum expresses insensitivity to iron-deficiency during P0-P10 (Pinero et al., 2000). Therefore, the onset of iron-deficiency during development is dependent upon the developmental stage and the brain region of interest (Pinero et al., 2000; Beard et al., 2003a). In addition, the stage at which iron-deficiency was induced in the mother (dam) is of great importance and can have dramatic consequences upon the offspring. For example, it has been shown that a reduced neonatal size occurs when irondeficiency was induced prior to and throughout pregnancy in rat dams, when compared to 
dams maintained on a control diet (Gambling et al., 2004). Furthermore, every stage of ID diet induction elicits a different effect, for example once iron-deficiency occurs at a neonatal stage the dopaminergic system cannot be fully restored (Beard and Connor, 2003). This effect can be shown by inducing iron deficiency prior to or during P8-P15, a critical stage when intersynaptic dopamine connections are made. The result of this iron-deficiency is an altered synaptic maturation within dopaminergic rich brain areas (Murrin and Zeng, 1990; Tarazi and Baldessarini, 2000).

\subsubsection{Importance of iron in homeostasis of the dopaminergic system}

Iron is of importance in dopamine synthesis as it serves as a co-factor for tyrosine hydroxylase (TH) during the rate limiting conversion step of L-Tyrosine to Levodopa (L-DOPA) in the norepinephrine (NE) synthetic pathway, in presynaptic dopamine neuron terminals. This conversion step requires enzymatic activity of $\mathrm{TH}$, which is dependent on ferrous iron $\left(\mathrm{Fe}^{2+}\right)$ as a cofactor. Subsequently, L-DOPA undergoes decarboxylation to produce dopamine (Nestler, 2001; Berger et al., 2002).

\subsubsection{Tyrosine hydroxylase}

Immunohistochemistry on RLS brains reveal a normal quantity of $\mathrm{TH}$ in the $\mathrm{SN}$, compared to controls (Connor et al., 2003). Similarly, in a rat model of iron-deficiency showing a 40\% reduction in brain iron concentration, no change in iron-dependent enzymes was noted. These enzymes were tryptophan hydroxylase, monoamine oxidase, cytochrome c oxidase, succinate dehydrogenase and TH (Youdim et al., 1983; Beard and Connor, 2003). In addition, another study revealed the circadian TH levels to closely match the level of dopamine in the mouse brain, which peaks at midnight and reaches a minimum level by 04:00hr (Huie et al., 1989). The messenger ribonucleic acid (mRNA) level of TH in rat SN pars compacta shows a decrease at 1 hour prior to the dark period and a steady increase from 04:00hr-16:00hr (Weber et al., 2004).

\subsubsection{Dopamine transporter and receptors}

Iron certainly induces a dynamic change in proteins involved in dopamine mediated function (Beard et al., 2003a), hence subsequently altering the dopaminergic system. The dopamine transporter (DAT) is a member of a group called the $\mathrm{Na}^{+} / \mathrm{Cl}^{-}$-dependent transporters (Rang, 1999). The $\mathrm{Na}^{+}$cotransporter is involved in reuptake of approximately $80 \%$ extracellular dopamine (Ciliax et al., 1995; Erikson et al., 2000). Upon increased dopamine receptor 2 (D2) agonist occupancy, the activity of DAT is increased and so is the reuptake clearance of synaptic dopamine (Meiergerd et al., 1993; Dickinson et al., 1999). Therefore, subsequent 
downregulation of the presynaptic D2 receptor may reduce DAT activity (Erikson et al., 2001), as the D2 receptor functions in a feedback loop to regulate DAT expression (Meiergerd et al., 1993; Dickinson et al., 1999). In addition, iron-deficiency can cause a dysfunction of the dopaminergic system by decreasing D2 receptor expression resulting in an increased dopamine concentration, as observed in the caudate-putamen (Beard et al., 1994; Nelson et al., 1997), and a reduced expression of DAT (Erikson et al., 2000). This function was confirmed in D2 knock-out $(\mathrm{k} / \mathrm{o})$ mice, which showed increased extracelluar dopamine as a result of reduced dopamine clearance due to the reduced function of DAT (Dickinson et al., 1999). ID male rats showed a $20-40 \%$ reduction in DAT residing in the caudate putamen, SN and NAcc (Burhans et al., 2005). Iron-deficiency without accompanying anaemia (Nelson et al., 1997), shows a reduced dopamine receptor 1 (D1), D2 and DAT expression in the caudate-putamen (Ashkenazi et al., 1982; Youdim et al., 1984; Erikson et al., 2000), where at the NAcc only a reduced expression of D2 and DAT was observed (Beard et al., 2002). Some of these transporter changes were predominantly shown in male as opposed to female rats and are reversible upon iron supplementation (Burhans et al., 2005).

\subsubsection{Involvement of other neurotransmitters}

Other than dopamine, several other catecholamines are predicted to be altered in-vivo during a state of iron-deficiency and therefore may participate in human RLS. These are epinephrine and NE, which along with dopamine simultaneously show a reduced peripheral concentration during the night time period (Sowers and Vlachakis, 1984). In addition, it has been suggested that the circadian levels of these catecholamines vary according to sleep/wake cycles (Sowers and Vlachakis, 1984).

The PLMS symptoms associated with RLS which cause sleep arousal are believed to involve the autonomic nervous system (ANS) (Winkelman, 1999), suggesting other than norepinephrine, the autonomic neurotransmitter acetylcholine (Rang, 1999) may play an important function.

\subsubsection{Choline acetyltransferase}

Acetyl-coenzyme A (Acetyl-CoA) and choline are converted into acetylcholine (ACh) through the enzyme activity of choline acetyltransferase (ChAT) (Wu and Hersh, 1994) and uptaken into synaptic vesicles by the aid of the vesicular acetylcholine transporter (VAChT) (Eiden, 1998). Choline availability rather than ChAT activity, is believed to be the rate-determining step in ACh synthesis (Blusztajn and Wurtman, 1983; Ferguson et al., 2003). Acetyl-CoA and choline availability influence the activity of this enzyme. Similarly to VAChT, ChAT is a marker of cholinergic neurons. This cytosolic enzyme is localised exclusively in cholinergic 
nerve terminals and is involved in acetylation of free choline by transferring the acetyl groups from acetyl-CoA, producing ACh. Following synthesis, ACh is then stored in vesicles prior to release from presynaptic terminals by means of presynaptic membrane vesicle docking and fusion, followed by exocytosis (Rang, 1999). Spinal motoneurons are strongly immunoreactive for ChAT and therefore this enzyme is of importance in ACh synthesis at spinal motor regions. In addition, the presence of a functioning cholinergic system determines the development of locomotor circuitry at the spinal level (Myers et al., 2005). Furthermore, as $\mathrm{ACh}$ is observed in spinal motoneurons, suggests the possible involvement that ChAT activity may have upon spontaneous locomotor activity, which is observed during early stages of embryonic spinal cord development (Myers et al., 2005).

\subsubsection{Function of myelin proteins during iron-deficiency}

Investigating the effects iron-deficiency has upon myelin formation and structure has been extensively studied in rodents (Connor and Menzies, 1990). Iron acts as a co-factor in cholesterol and lipid synthesis, where brain lipid composition may become altered in irondeficiency, therefore, the fatty acid composition of the brain becomes disrupted (Oloyede et al., 1992) causing hypomyelination (Yu et al., 1986). Oligodendrocytes contain high levels of iron, Tf and ferritin (Connor, 1994) which are important for myelination (Kwik-Uribe et al., 2000a). Suppressing oligodendrocytic maturation is accompanied by brain accumulation of iron, Tf and its receptor. Therefore, acquiring brain iron through the transferrin-receptor (TfTfR) complex is important in maturation of oligodendrocytes and subsequent myelination. In addition, oligodendrocytes are important in maintaining brain iron at a cellular level (Connor, 1994).

Iron has been shown in clinical and animal rodent studies to have an important role in myelination (Yu et al., 1986; Connor and Menzies, 1990; Ortiz et al., 2004). This was proven by observing a parallel increase in myelinogenesis during the time-point coinciding with heightened brain iron uptake (Ward et al., 2007). Moreover, inducing iron-deficiency in rats during development, showed a prolonged reduction of myelin levels at 6 months of age and formation of immature myelin (Erikson et al., 1997). A decrease in phospholipids and proteins associated with myelin compaction were also observed, despite the presence of normal iron content in the brain and myelin. This suggests the importance in the stage of iron-deficiency onset during development (Lozoff et al., 2006), where an overall decreased brain iron content has been connected to hypomyelination ( $\mathrm{Yu}$ et al., 1986), a dysfunctional dopaminergic system (Nelson et al., 1997) and impaired neuromaturation (Erikson et al., 2001). 


\subsubsection{Implications of the sirtuin 2 protein in myelin}

The origin of the mammalian sirtuin family is derived from yeast Saccharomyces cervisiae as the silent information regulator 2 protein (Sir2p), which has been characterised in humans (Frye, 1999; Frye, 2000). Sirtuin 2 (SIRT2) is expressed as two variants. Firstly, variant 2 (v2) has prevalence in myelin and variant 1 (v1) has widespread distribution throughout the brain and is not restricted to myelin (Werner et al., 2007). Generally a higher sirtuin protein expression is observable in the brain, despite the broad distribution of the seven family members throughout all human tissue (Frye, 1999; Michishita et al., 2005). In particular, SIRT2 shows a higher expression level in the developing foetal brain (Yamamoto et al., 2007), where the pattern of SIRT2 is restricted to oligodendrocytes in the mammalian central nervous system (CNS), embedded in the outer non-compact layer of the myelin sheath (Bifulco et al., 2002; Lappe-Siefke et al., 2003; Li et al., 2007; Werner et al., 2007). SIRT2 overexpression is associated with diminished maturation of oligodendrocytes, notably tubulin acetylation and arbourisation (Fulco et al., 2003; Li et al., 2007).

Werner and colleagues (2007) showed the importance of proteolipid protein/DM20 (PLP/DM20) co-expression with SIRT2 (v2) in allowing transport into the myelin (Werner et al., 2007). During oligodendrocytic development, SIRT2 participates in regulating microtubule dynamics (Li et al., 2007) and therefore may be implicated in hindering axon degeneration and normalising axon-glia communication (Werner et al., 2007). Particular excitement eludes the SIRT2 protein as a potential target for the treatment of alpha-synuclein ( $\alpha$-Syn) induced toxicity in Parkinson's, Alzheimer's and Huntington's diseases, where SIRT2 expression was shown to be elevated in a Parkinson's disease model (Outeiro et al., 2007).

\subsection{Central nervous involvement in RLS}

Several structures are believed to be involved in producing RLS symptoms. As the dopaminergic system acts as a central cause of these symptoms, has meant that many supraspinal regions have been studied, including the SN, red nucleus and A10-A14 nuclei. From these nuclei, the diencephalic A11 nucleus is known to be an important pathway and source of descending dopaminergic innervation into the spinal cord (Skagerberg and Lindvall, 1985). These dopaminergic fibres extent through the entire spinal cord and are known to innervate the intermediolateral nucleus (IML) and all rexed laminae of both dorsal and ventral horns of the spinal cord (Lindvall et al., 1983; Skagerberg and Lindvall, 1985). Recent lesioning studies of the A11 nucleus, describe this structure to be central in producing classic RLS symptomatology (Qu et al., 2007; Zhao et al., 2007). Therefore, an adequate dopaminergic system appears important in order to maintain sensorimotor integrity. 


\subsubsection{Supraspinal regions}

The nervous system is under a strong circadian influence by the suprachiasmatic nuclei (SCN) (Moore, 1996). This structure is known to form a connection with the diencephalic A11 nuclei (Reuss, 1996). In addition, three different regions are believed to increase hindlimb movements and restlessness during sleep. These include the rostrolateral, rostromedial and caudal regions of the ventral mesopontine junction (VMPJ) (Lai et al., 2008). Furthermore, the NAcc may also be involved as it has been shown to change during iron-deficiency (Yehuda and Youdim, 1989; Erikson et al., 2001; Beard and Connor, 2003).

Supraspinal structures which alter the sensory system of the dorsal spinal cord are illustrated in Fig. 1.1. Spinothalamic tracts project from dorsal spinal laminae I and V to the thalamus. This projectory pathway also goes through spinobulbar pathways onwards to subcortical regions. These regions include the parabrachial nucleus which relays nociceptive information onto the amygdala and hypothalamus, the rostral ventromedial medulla (RVMM) and the periaqueductal grey (PAG). Other brain regions which show a bidirectional influence on pain sensation include the amygdala; PAG; RVMM; prefrontal, anterior cingulate, and insular cortices; periventricular and posterolateral hypothalamus; and dorsolateral pons (Basbaum and Fields, 1984; Millan, 2002). These supraspinal structures are known to facilitate or inhibit the transmission of nociceptive information at the dorsal horn (Basbaum and Fields, 1984; Gebhart, 2004). 


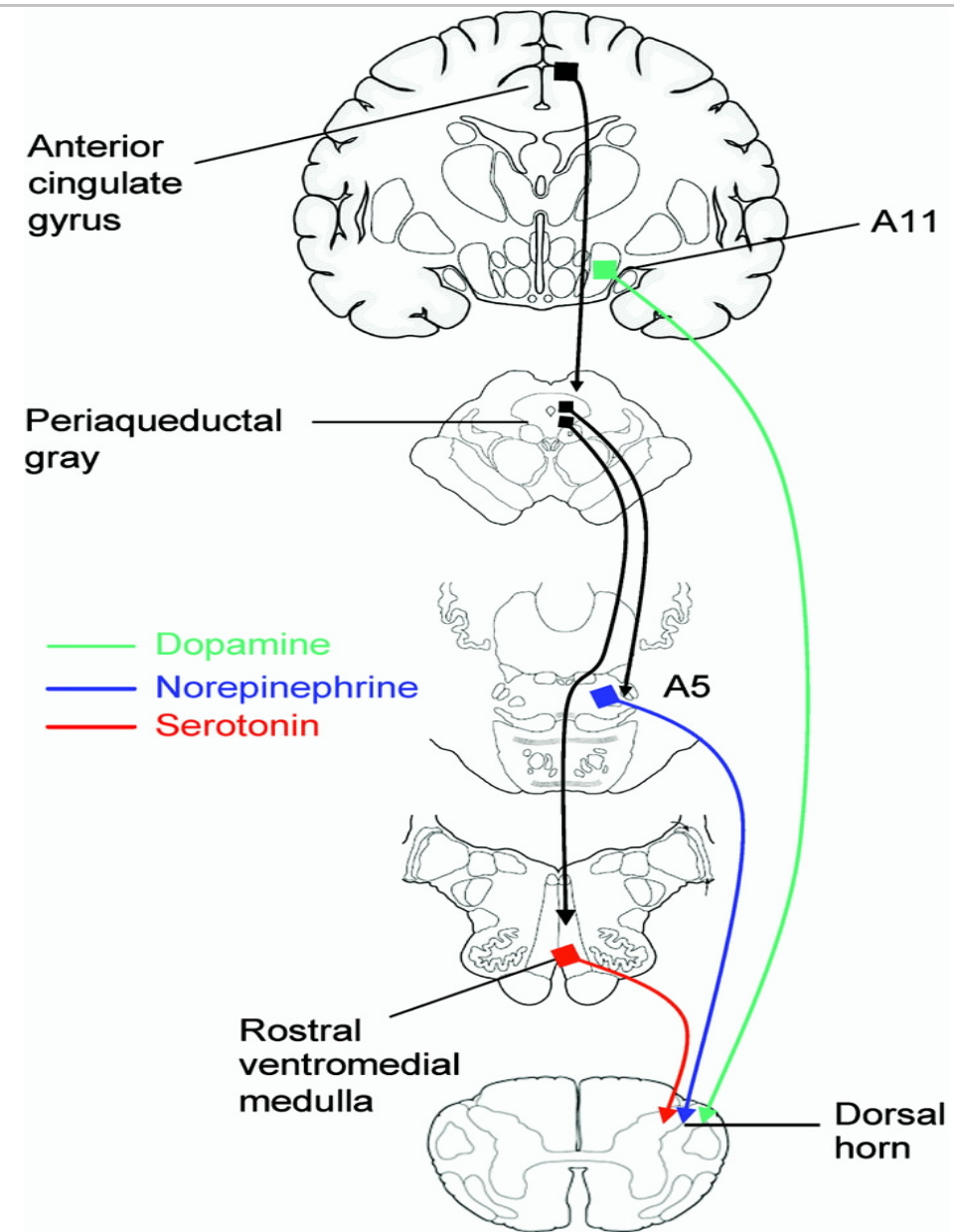

Fig. 1.1. Monoaminergic pathways originating from supraspinal regions and innervating the dorsal horn. This figure illustrates monoaminergic pathways innervating the dorsal horn of the spinal cord from the following supraspinal structures; cingulated gyrus, periaqueductal gray, dorsolateral pontine tegmentum, and ventromedial medulla. The result of transmission through these monoaminergic pathways is either a pronociceptive or antinociceptive effect. Taken from a review by (Benarroch, 2008).

The three monoamines depicted in the figure above (Fig. 1.1) are involved in modulating nociceptive information at the dorsal horn and arise from different supraspinal structures. Firstly, dopamine originates from the A11 nuclei, norepinephrine from several cell groups (A5, A6 (locus ceruleus), and A7 groups) in the pontine tegmentum (Millan, 2002) and finally the serotonin pathway arising from the RVMM. All three monoaminergic pathways have important functions in modulating pain and express numerous receptor subtypes at the dorsal horn region (Benarroch, 2008).

\subsubsection{Dorsal horn region of the spinal cord}

The A11 projects downwards through the spinal cord and innervates all rexed laminae, however it shows a greater innervation upon superficial laminae of the dorsal horn, namely superficial lamina I and the substantia gelatinosa lamina II regions (Lindvall et al., 1983; Skagerberg and Lindvall, 1985; Millan, 2002).

The dorsal horn is made up of different laminae. The laminae of particular importance when relaying nociceptive to supraspinal structures are I and II. Different primary afferent 
fibres innervate these two laminae, these are the A delta $(\mathrm{A} \delta)$ and the C-fibres. They both release various neurotransmitters and modulators which act upon corresponding receptors (see Fig. 1.2), for example glutamate acts upon N-methyl-D-aspartic acid (NMDA) receptors (Nestler, 2001). Originating from supraspinal structures (see Fig. 1.1), monoamine pathways relaying dopamine (DA), NE and serotonin (5HT) all innervate the superficial dorsal laminae, as illustrated below (see Fig. 1.2, "FROM BRAIN"). Upon release, these monoamines act upon various receptor subtypes for DA (D1, D2, D3), NE ( $\alpha 2 \mathrm{~A})$ and 5HT (5HT1A, 5HT2, 5HT3) (Peterfreund et al., 1995; Xie et al., 1998; Nestler, 2001; Millan, 2002). Extracellular concentrations of the monoamines are regulated by presynaptic transporters, DAT, norepinephrine transporter (NET) and serotonin transporter (SERT) (Nestler, 2001). In addition, other neurotransmitters including; glycine, ACh and gamma-aminobutyric acid (GABA) are also present, where ACh has been shown to act upon the muscarinic receptor 3 (M3) at the spinal cord which is known to be involved in nociception during the second phase of the formalin-induced pain test (Honda et al., 2000). Interestingly, this pain test is known to involve C-fibres (Dickenson and Sullivan, 1987) which are important primary afferents for relaying peripheral nociception information to the spinal level.

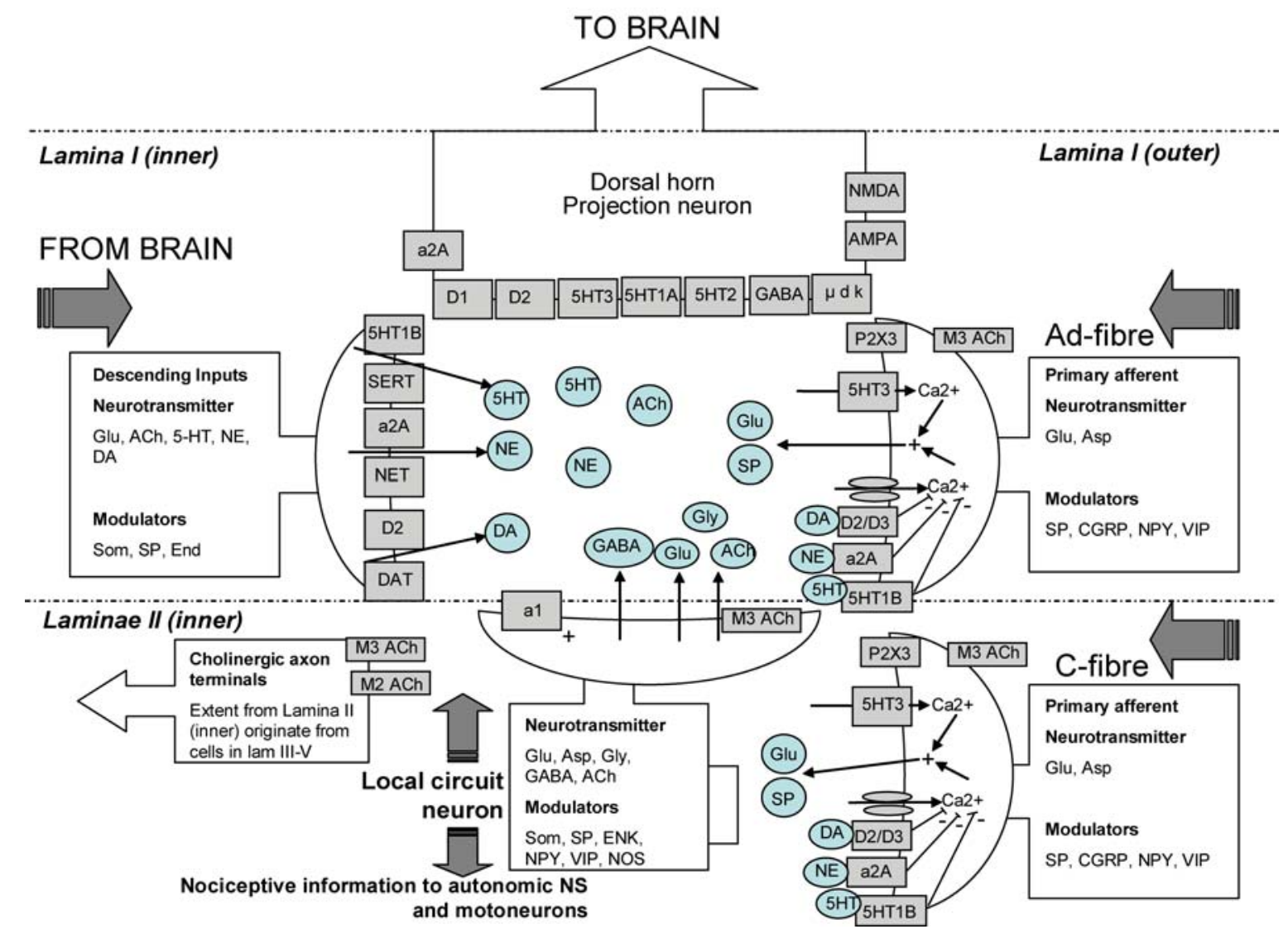

Fig. 1.2. Overview of neurotransmitters, modulators and receptors involved in relaying nociceptive information at the dorsal horn. This figure represents the neuroanatomical structure of $\mathrm{A} \delta$ and $\mathrm{C}$-fibres in relation to either lamina I or II. Moreover, the involvement of the descending inputs which originate from supraspinal structures as previously shown (Fig. 1.1) innervate the superficial region of the sensory dorsal horn (FROM BRAIN). Various receptors are illustrated, and neurotransmitters which directly interact with receptors residing upon the dorsal horn projection neurons, which subsequently relay nociceptive information supraspinally (TO BRAIN). 


\subsubsection{Ventral horn region of the spinal cord}

In addition to the high density innervation of A11 projections at the dorsal horn, a substantial innervation is observed at the ventral horn and the IML, where the latter acts as the spinal origin for sympathetic preganglionic pathways (Lindvall et al., 1983; Skagerberg and Lindvall, 1985). Furthermore, between the lower thoracic and upper lumbar regions of the spinal cord lies an important centre which has been known to have a profound role in generating locomotor activity (Wilson, 1961). This region is known as the central pattern generator (CPG). Once a movement is initiated, the CPG is activated where midbrain and brainstem descending locomotor inputs maintain its activity (Lafreniere-Roula and McCrea, 2005). Locomotor CPG neurons function in generating timing, rhythm and coordination of muscles, therefore controlling motor output at a spinal level. All vertebrates have this network of spinal interneurons which makes up the structure of the CPG (Kiehn, 2006).

Studies in neonate rat spinal cord, show that the CPG is associated with hindlimb movements and is located at lumbar segments 1 and 2 (L1-L2) and that only through these segments can cross-excitatory and inhibitory pathways pass from side to side (Cazalets et al., 1995; Kremer and Lev-Tov, 1997). The CPG present in the rostral region of the lumbar cord are more greatly excited and are suggested to be involved in evaluating the initiation and cycle time of locomotion (Kremer and Lev-Tov, 1997). Interestingly, muscle activity in the isolated neonate rat spinal cord recorded by ventral root activity, has been shown to be activated upon application of 5-HT, ACh, DA (Cazalets et al., 1992; Cowley and Schmidt, 1994; Kiehn and Kjaerulff, 1996) and modulated by NE (Kiehn and Kjaerulff, 1998). With regards to ACh, the cholinergic system and ChAT have previously been demonstrated in producing spontaneous activity (Misgeld et al., 2002; Brandon et al., 2003; Myers et al., 2005), as structurally the cholinergic system is prominent in this region as shown by large cholinergic C-terminals contacting motoneurons (Ferguson et al., 2003). This shows the importance of certain neurotransmitters upon CPG motor output (Butt et al., 2002). Further studies on the rat CPG show the presence of rhythmic activity extending rostrally through the lumbar region into the caudal thoracic segment (Kjaerulff and Kiehn, 1996; Cowley and Schmidt, 1997; Kremer and Lev-Tov, 1997; Cazalets and Bertrand, 2000). The rostral-caudal gradient representative of the lumbothoracic cord region which are involved in generating rhythmicity, are concentrated specifically at L1 and L2 segments (Butt et al., 2002) and at thoracic segment 13 (T13), where the lower lumbar segments have no function in rhythmicity (Kiehn, 2006).

Colocalised at the spinal cord are motoneurons and interneurons, which are important in motor control (Shepherd, 1994). Depending upon the area of the spinal cord, different interneuron 
populations are present and reside in various laminae of the spinal cord, as illustrated below (Fig. 1.3). Renshaw cells (RCs) and Ia interneurons (Ia-INs) which are spinal inhibitory interneurons project to motoneurons and are believed to be important in flexor-extensor coordination (McCrea et al., 1980; Pratt and Jordan, 1987). Additionally, ipsilateral inhibitory pathways have also been shown to be of importance (Kjaerulff and Kiehn, 1997; Bonnot et al., 2002). The involvement of RCs in the CPG is mostly associated with fine tuning of motoneuronal firing and burst termination, where the Ia-INs function in inhibiting rhythmic activity of motoneurons initiated in locomotion, therefore, these interneurons serve in flexorextensor coordination (McCrea et al., 1980; Pratt and Jordan, 1987; Kiehn, 2006). Loss of rhythmogenicity in group I-activated extensor interneurons results in tonic activation of flexor motoneurons and decreased locomotor rhythm, thus a decrease flexor CPG activity. This implies these interneurons are imperative during locomotion driven extensor motoneuron activity (Kiehn, 2006).
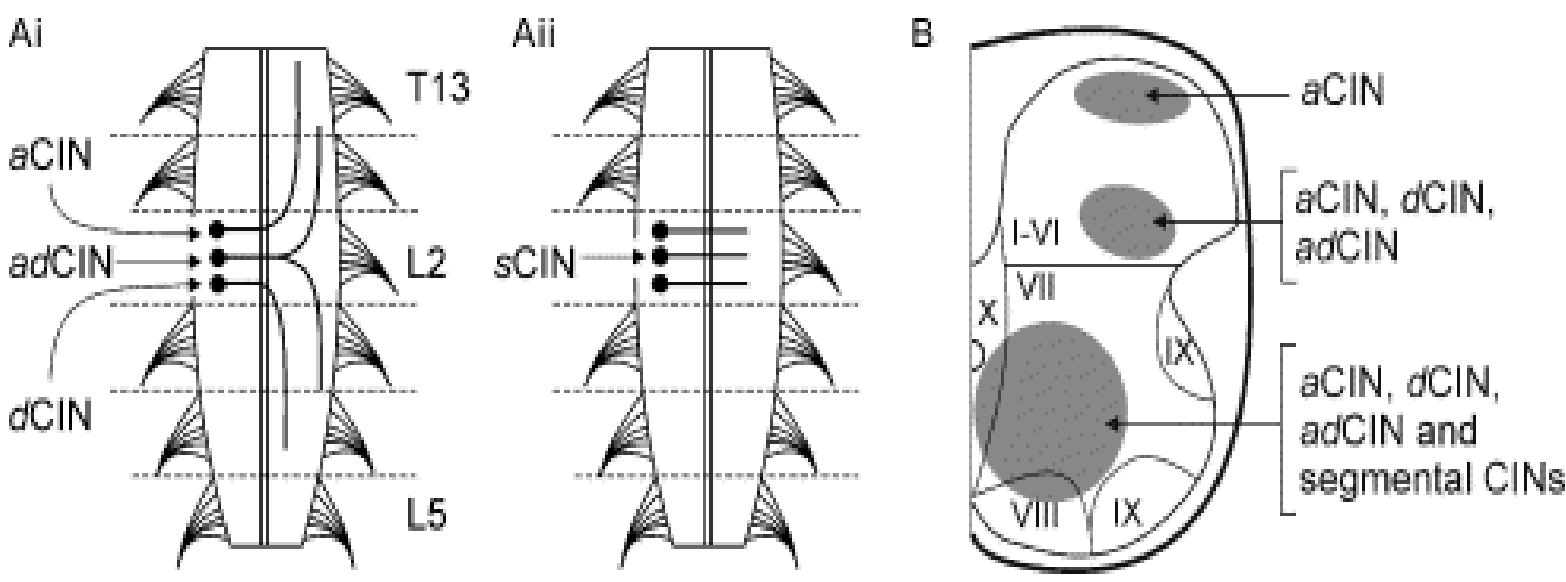

Fig. 1.3. Distribution of interneuron groups at T13-L2 spinal segements. This figure illustrates the anatomical distribution of rat lumbar spinal cord commissural interneurons (CINs). Divided into two main groups, firstly long-range as shown in (Ai), this includes 1) pure descending CINs ( $d \mathrm{CINs}), 2)$ pure ascending CINs ( $a \mathrm{CINs})$, 3) both ascending/descending CINs ( $a d C I N s)$. Secondly, short-range as shown in (Aii) project only in one spinal segment. (B) shows the broad distribution of the different CINs in both dorsal and ventral cord, where the dark grey regions represent locomotor-generating ventromedial areas. Taken from a review by (Kiehn and Butt, 2003).

The involvement of the CPG in RLS has been postulated, based on the occurrence of both RLS and PLMs in paraplegic patients (de Mello et al., 1996; Lee et al., 1996). A patient with a transected cervical region of the spinal cord still produced rhythmic movements at the legs stimulated by flexor reflex afferents (Bussel et al., 1988; Barriere et al., 2005). Similarly in leg restlessness, arm restlessness can occur along with periodic arm movements (PAM), where the spinal pattern generator at the cervical cord region is central to this effect (Zehr et al., 2004). Activation of cutaneous afferents is shown to initiate both arm and leg spinal networks during walking, suggesting a coupling between upper and lower limbs (Barriere et al., 2005). 


\subsection{Animal models of RLS}

\subsubsection{Current animal models of RLS}

Several different animal models have been proposed (see Table 1.1) with the aim in reproducing RLS symptoms. The complexity of producing a valid animal model comes from trying to recreate more than one symptom, i.e. both a sensory and motor component, as described in humans. The added difficulty is ensuring symptom onset occurs in a circadian manner, therefore at specific time-points and not outbursts of symptoms which occur all the time, throughout daytime and night time. Certain studies have elucidated a circadian onset of symptoms relating to humans, by using electromyographic (EMG) and/or electroencephalographic (EEG) recordings, showing increased wakefulness prior to the start of the light cycle, therefore corresponding to the evening, i.e. prior to sleep in humans (Dean et al., 2006). This model, in addition to several others as summarised in Table 1.1, commonly show the same trend and that is an increase in hindlimb movements and wakefulness during sleep. Other studies also make use of activity chambers and observe the nocturnal animal's activity only for a short period from the total $24 \mathrm{hr}$ circadian cycle. In two separate experiments, changes in motor activity were identified at a circadian time-point noncomparable to the onset of symptoms in humans, 09:00hr-12:00hr light phase, or 09:00hr12:00hr dark phase, as previously described in a review (Paulus et al., 2007b). The most common type of method for inducing RLS-like symptoms in an animal is lesioning by 6hydroxydopamine (6-OHDA) or neurotoxic NMDA, either at the diencephalic A11 or the VMPJ region, respectively. Lesioning at these sites indicate the importance of the dopaminergic system and these regions in inducing changes in motor activity, however inadequate testing (both sensory and motor), and for certain experiments inappropriate periods of testing (Qu et al., 2007; Zhao et al., 2007) mask the true effects of reducing the A11 dopaminergic output and the ramifications it may have upon sensorimotor function.

In the future, one would expect more emphasis on a genetic approach in producing a suitable animal model of RLS based from recent genetic findings in humans, which identified 4 genetic variants; MEIS1, BTBD9, MAP2K5/LBXCOR1 and PTPRD (Stefansson et al., 2007; Winkelmann et al., 2007; Schormair et al., 2008). Importantly, future models should also focus on identifying changes in sensory symptoms in addition to changes in motor symptoms, as there remains a lack of behavioural studies showing such changes (see Table 1.1) which can be compared to those in humans. However, current unpublished studies do illustrate both sensory/motor changes, in addition to identifying a circadian onset of heightened motor activity. Furthermore, these changes were also examined in dopamime receptor 3 (D3) k/o mice (Dowling et al., unpublished data). 


\begin{tabular}{|c|c|c|c|c|c|c|c|}
\hline Model & $\frac{\text { Genus / Strain / }}{\underline{\text { Sex }}}$ & Approach & $\frac{\text { Sensory }}{\underline{\text { Tests }}}$ & $\frac{\text { Motor }}{\underline{\text { Tests }}}$ & $\frac{\text { Circadian }}{\text { Onset }}$ & Other Observations & Authors \\
\hline \multirow[t]{2}{*}{ Genetic } & $\overline{M u s / B l / 6}$ & $\begin{array}{l}\text { Genetic knockout of } \\
\text { dopamine } 3 \text { receptor } \\
\left(\mathrm{D}^{-/}\right)\end{array}$ & - & $\begin{array}{l}\text { Open field. } \\
\text { Light phase- Hyperactive } \\
\text { (13:00hr-18:00hr) }\end{array}$ & - & $\begin{array}{l}\text { 1) No gross developmental defects. } \\
\text { Normal gait and coordination. }\end{array}$ & (Accili et al., 1996) \\
\hline & Mus / Bl/6 & $\mathrm{D}^{-/-}$in-vitro studies & - & $\begin{array}{l}\text { Dopamine decreases spinal } \\
\text { excitability in WT and } \\
\text { increases in } \mathrm{D} 3^{-/ /} \text {mice }\end{array}$ & - & - & $\begin{array}{l}\text { (Clemens and } \\
\text { Hochman, 2004) }\end{array}$ \\
\hline \multirow[t]{6}{*}{ Lesioning } & Mus / Bl/6 / male & $\begin{array}{l}\text { 6-OHDA lesion of } \\
\text { A11 neurons }\end{array}$ & - & $\begin{array}{l}\text { Activity chamber } \\
\text { Dark phase-Increase } \\
\text { activity (09:00hr-12:00hr) }\end{array}$ & - & $\begin{array}{l}\text { 1) After } 8 \text { weeks diet spinal iron- } \\
\text { level content reduced. } \\
\text { 2) Dopamine level is reduced in } \\
\text { lumbar spinal cord. } \\
\text { 3) Lumbar spinal cord-Increase D1 } \\
\text { mRNA, but lesioning causes } \\
\text { decrease in D2 and D3 mRNA. }\end{array}$ & (Zhao et al., 2007) \\
\hline & Mus / Bl/6 / male & $\begin{array}{l}\text { 6-OHDA lesion of } \\
\text { A11 neurons }\end{array}$ & - & $\begin{array}{l}\text { Activity chamber } \\
\text { Light phase-Increase } \\
\text { activity (09:00hr-12:00hr) }\end{array}$ & - & $\begin{array}{l}\text { 1) After } 4 \text { weeks diet serum iron- } \\
\text { level reduced in non-lesioned mice, } \\
\text { brain and spinal cord after } 8 \text { weeks } \\
\text { diet. }\end{array}$ & (Qu et al., 2007) \\
\hline & Felus / female & $\begin{array}{l}\text { NMDA lesions of } \\
\text { the VMPJ }\end{array}$ & - & $\begin{array}{l}\text { EMG } \\
\text { Increased motor } \\
\text { hyperactivity }\end{array}$ & $\begin{array}{l}\text { EEG } \\
\text { Onset of hindlimb } \\
\text { movements during } \\
\text { sleep. Increased } \\
\text { wakefulness } \\
\end{array}$ & $\begin{array}{l}\text { 1) No correlation with loss of } \\
\text { dopamine neurons from lesion site } \\
\text { and sleep pattern. }\end{array}$ & (Lai et al., 2008) \\
\hline & $\begin{array}{l}\text { Rattus / Sprague- } \\
\text { Dawley / female }\end{array}$ & $\begin{array}{l}\text { 6-OHDA lesion of } \\
\text { A11 neurons }\end{array}$ & - & $\begin{array}{l}\text { Activity chamber } \\
\text { Light phase-Increase } \\
\text { activity, reduced sleep } \\
\text { time }(08: 00 \mathrm{hr}, 10: 00 \mathrm{hr} \text {, } \\
\text { 14:00hr) }\end{array}$ & - & $\begin{array}{l}\text { 1) } \mathrm{TH} \text { neurons reduced in } \mathrm{A} 11 \\
\text { region, however unchanged in } \mathrm{SN} \\
\text { and ventral tegmental area. }\end{array}$ & (Ondo et al., 2000) \\
\hline & $\begin{array}{l}\begin{array}{l}\text { Rattus / Wistar / } \\
\text { male }\end{array} \\
\end{array}$ & $\begin{array}{l}\text { Spinal cord injury } \\
\text { T9 segment }\end{array}$ & - & $\begin{array}{l}\text { EMG } \\
\text { Increased hindlimb } \\
\text { movements and cervical } \\
\text { musculature }\end{array}$ & $\begin{array}{l}\text { EEG } \\
\text { Onset of hindlimb } \\
\text { movements during } \\
\text { sleep }\end{array}$ & $\begin{array}{l}\text { 1) Histology showed morphologic } \\
\text { changes of spinal cord after surgical } \\
\text { injury at T9 }\end{array}$ & (Esteves et al., 2004) \\
\hline & $\begin{array}{l}\text { Rattus / Wistar / } \\
\text { male }\end{array}$ & $\begin{array}{l}\text { 6-OHDA lesion of } \\
\text { A10 neurons }\end{array}$ & - & $\begin{array}{l}\text { Voluntary wheel-running } \\
\text { activity reduced in dark } \\
\text { phase but longer activity } \\
\text { (early light phase) }\end{array}$ & - & - & $\begin{array}{l}\text { (Isobe and Nishino, } \\
\text { 2001) }\end{array}$ \\
\hline
\end{tabular}

Table 1.1. Existing animal models relating to RLS. Shows all the current animal models and the strategies used to induce symptoms, and the tests for measuring the sensory, motor and circadian facits which are commonly associated in human RLS. The model type gives an idea of the different existing animal models performed in different species, indicated by their genus and strain. Sensory and motor symptoms were identified in the model by certain tests, similarly for the circadian involvement, i.e. whether they show there to be a specific circadian onset characteristically during later stages of dark cycle in nocturnal animals, which corresponds to late stages of the light cycle in humans. The column entitled "other observations" describes other changes that were observed. Finally, the author that created the model is listed. The minus sign (-) represents that this was not performed. 


\begin{tabular}{|c|c|c|c|c|c|c|c|}
\hline Model & $\frac{\text { Genus / Strain / }}{\underline{\text { Sex }}}$ & Approach & $\frac{\text { Sensory }}{\underline{\text { Tests }}}$ & $\frac{\text { Motor }}{\underline{\text { Tests }}}$ & $\frac{\text { Circadian }}{\text { Onset }}$ & Other Observations & Authors \\
\hline \multirow[t]{4}{*}{$\begin{array}{c}\text { Dietary } \\
\text { Intervention }\end{array}$} & Mus / Bl/6 / male & ID diet (4-8 weeks) & - & $\begin{array}{l}\text { Activity chamber } \\
\text { Dark phase-Increase } \\
\text { activity }(09: 00 \mathrm{hr}-12: 00 \mathrm{hr})\end{array}$ & - & $\begin{array}{l}\text { 1) After } 8 \text { weeks diet haemoglobin } \\
\text { levels reduced and spinal iron-level } \\
\text { content reduced. } \\
\text { 2) TH neurons unchanged in A11- } \\
\text { A14 and SN. } \\
\text { 3) No change in dopamine and } \\
\text { serotonin level in lumbar spinal } \\
\text { cord. } \\
\text { 4) Lumbar spinal cord-Increase D1 } \\
\text { mRNA and no change in D2 and } \\
\text { D3. }\end{array}$ & (Zhao et al., 2007) \\
\hline & Mus / Bl/6 / male & ID diet (4-8 weeks) & - & $\begin{array}{l}\text { Activity chamber } \\
\text { Light phase-Increase } \\
\text { activity }(09: 00 \mathrm{hr}-12: 00 \mathrm{hr})\end{array}$ & - & $\begin{array}{l}\text { 1) After } 4 \text { weeks diet serum iron- } \\
\text { level reduced in non-lesioned mice, } \\
\text { brain and spinal cord after } 8 \text { weeks } \\
\text { diet } \\
\text { 2) TH neurons unchanged in A11- } \\
\text { A14 and SN. }\end{array}$ & (Qu et al., 2007) \\
\hline & Mus / Bl/6 / male & ID diet (3-5 weeks) & - & - & $\begin{array}{l}\text { EMG/EEG } \\
\text { Reduced sleep } \\
\text { and increased } \\
\text { wakefulness at } 4 \\
\text { hours prior to } \\
\text { light phase (sleep } \\
\text { stage) }\end{array}$ & - & (Dean et al., 2006) \\
\hline & $\begin{array}{l}\text { Rattus / Sprague- } \\
\text { Dawley / male }\end{array}$ & ID diet (4-8 weeks) & - & $\begin{array}{l}\text { Wheel-running activity } \\
\text { shows reversed activity } \\
\text { cycle for ID rats }\end{array}$ & $\begin{array}{l}\text { Onset of elevated } \\
\text { activity during } \\
\text { light phase } \\
\text { compared to dark } \\
\text { phase in ID rats }\end{array}$ & - & (Youdim et al., 1981) \\
\hline Aged effect & $\begin{array}{l}\text { Rattus / Wistar / } \\
\text { male }\end{array}$ & $\begin{array}{l}\text { Ageing effect upon } \\
\text { hindlimb activity } \\
\text { and sleep }\end{array}$ & - & $\begin{array}{l}\text { EMG } \\
\text { Increased spontaneous } \\
\text { periodic hindlimb } \\
\text { movements in aged rats }\end{array}$ & $\begin{array}{l}\text { EEG } \\
\text { Onset of hindlimb } \\
\text { movements } \\
\text { during sleep }\end{array}$ & - & (Baier et al., 2002) \\
\hline
\end{tabular}

Table 1.1. continued. Existing animal models relating to RLS. This table is a continuation of the previous table with the added exception of showing different models e.g. dietary intervention and ageing to induce RLS-like symptoms. The minus sign (-) represents that this was not performed. 


\subsubsection{Approach in designing an animal model of RLS}

After becoming familiar with the existing animal models of RLS (see Table 1.1), it is easy to identify the lack of progress in identifying certain symptoms, for example sensory symptoms. However, trying to recreate the symptoms of a disorder in an animal is no easy task and often results in partially achieving the initial aims. The main aim when creating or using animal tests for accessing certain phenotypes should involve a minimum level of interference and handling of the animal during the experiment, otherwise any chance of a change between groups may become masked by stress of the animal or the complex nature of the test. Before starting in designing a novel animal model, the true nature of the disorder one wishes to recreate in an animal must be understood.

RLS is characterised by an unpleasant and painful sensation of the legs, which occurs prior to or during rest periods in the evening (Walters, 1995; Allen and Earley, 2001). Many causative and risk factors are associated with RLS, one of which is iron-deficiency (Ekbom, 1960; Sun et al., 1998). This is assumed to be a contributing factor in RLS, as intravenous iron alleviates RLS symptoms in patients (Earley et al., 2004; Wang et al., 2009). Therefore, inducing iron-deficiency by an ID diet represents a promising approach in establishing an animal model, which may show at least some clinical features of human RLS (Baier et al., 2007). Interestingly, iron-deficiency induces altered sleep-wake behaviour and increased wakefulness in mice subjected to ID diet (Dean et al., 2006). Other symptoms of RLS include impairment of temperature perception and $\mathrm{C}$-fibre function, the latter occurring predominantly in secondary RLS patients (Schattschneider et al., 2004). Furthermore, RLS patients possess an increased static hyperalgesia in their legs which is believed to result from A $\delta$-fibre sensitisation (Stiasny-Kolster et al., 2004b).

\subsubsection{The sensory component}

In light of these clinical findings, the formalin-test may prove useful in investigating the effects of ID diet on C-fibre function in mice. Initially established by Dubuisson and Dennis (1977), the formalin-test has proved an important analytical tool in assessing the effectiveness of analgesic drugs in a model of acute and prolonged inflammatory pain. This test is characterised by an inflammatory mechanism caused by the formalin injection (Dubuisson and Dennis, 1977; Dickenson and Sullivan, 1987), which induces an initial acute pain stimulus (Phase I) followed by chronic inflammatory pain lasting up to one hour (Phase II) (Dubuisson and Dennis, 1977; Malmberg and Yaksh, 1992). The initial acute phase represents the activation of both A $\delta$ - and C-fibre nerve endings (Heapy, 1987; Ishizaki et al., 1999) relaying a sharp and dull pain, respectively (Shepherd, 1994). The second phase represents the 
activation of $\mathrm{C}$-fibre nerve endings alone and is correlated with a central sensitisation (Yamamoto and Yaksh, 1992; Dorazil-Dudzik et al., 2004), where superficial dorsal horn neurons are prone to a lasting over-stimulation (Mendell, 1966). This central facilitation is paralleled by the level of c-Fos activation within specific laminae I/II of the dorsal horn, which is expressed alongside dynorphin upon intense pain inputs (Shepherd, 1994). Furthermore, an extensive literature describes the use of c-Fos immunohistochemistry after the formalin test, where during this pain test its expression has been suggested to promote formalin-induced pain (Hunter et al., 1995). The unique thing about the formalin-test is that upon injection of this chemical a quantifiable behavioural response is induced, which can be monitored and quantified without any handling or interference of the animal for upto 1 hour (Tjolsen et al., 1992). The final score is indicative of the amount of agrivation this chemical causes.

The hot-plate test is a measure of pain perception at the supraspinal level. Eddy and Leimbach., (1953) were the first to describe its use (Eddy and Leimbach, 1953). The response time to this thermal stimulus is measured from the time the mouse is placed upon the hot-plate surface until one of the following signs of behaviour are observed: 1) vocalization, 2) licking of hindpaw and 3) escape response (Gabra and Sirois, 2005). In contrast to the formalin-test, the hot-plate test induces an initial acute response to a thermal stimulus (Eddy and Leimbach, 1953 ) and is believed to involve myelinated $\mathrm{A} \delta$ - and to a lesser extent unmyelinated C-fibres (Shepherd, 1994; Frolich et al., 2005), which depends upon the temperature of the thermal stimulus (Kandel, 2000).

\subsubsection{The motor component and identifying the circadian onset}

The motor component which is descriptive of the syndrome's nomenclature, causes a repetitive leg movement termed PLMs and usually prevails prior to or during sleep, where it is termed periodic limb movements while awake (PLMWA) and PLMS, respectively (Walters, 1995; Allen and Earley, 2001).

The stereotypic activity of voluntary wheel-running is a measure of motor performance influenced by a circadian rhythm and was originally established by Stewart in 1898 (Stewart, 1898) who performed this test on rats. Wheel-running represents the animal's general activity, exploration, escape, play, body weight, hormonal status, adrenal activity and level of deprivation. The voluntary wheel-running detects increased activity caused by numerous factors, for example food deprivation (Treichler and Hall, 1962), environmental alterations (Strong, 1957; Treichler and Hall, 1962; Cornish and Mrosovsky, 1965) and hormonal influences (oestrous) (Finger, 1969). Compared to other forms of locomotor measuring devices, the wheel-running usage and subsequent capacity for activity is far greater than mazes, or activity chambers. Currently, no causative reason explains why running-activity 
occurs. A generally accepted notion is that wheel-running pattern is dependent upon the external circadian pacemaker activity. Moreover, all scenarios describe an internal causative factor, where the animal is motivated to use the wheel in a self-reward seeking manner (Sherwin, 1998). Voluntary wheel-running is a good model for observing exercise performance in animals (Shiga et al., 2003), therefore it can be used to determine the extent a special formulated diet has upon the animal's exercise capacity. For example exercise performance is hindered during severe iron deficiency (iron deficiency anaemia), due to reduced oxygen transport from lungs to the skeletal muscle (Ohira et al., 1981; Koziol et al., 1982), where in severe anaemia, endurance measured by the running time on a treadmill is drastically reduced (Koziol et al., 1982; Davies et al., 1984; Shiga et al., 2003).

The parameters obtained when using a voluntary wheel-running system are extensive and describe not only the animal's condition monitored by performance parameters (distance and velocity) but more importantly the level of activity (activity time and number of runs). This level of activity can be paralleled to the increased activity occurring in RLS patients prior to sleep. In addition, increased activity may also describe the effect of PLMs which often results in the person getting up and walking until the symptoms subside (Walters et al., 1991). A similar effect may also be elucidated by using the voluntary wheel-running system and subsequently observing any change in voluntary activity over the continuous $24 \mathrm{hr}$ recording period and more specifically at a time-point when symptoms occur in humans, therefore before the start of the light cycle in rodents.

\subsubsection{Direction of future animal models}

Currently, many models have been developed aimed at mimicking similar symptoms to that of human RLS. These include lesioning, genetic, pharmacological (Ondo et al., 2000; Baier et al., 2002; Clemens and Hochman, 2004; Esteves et al., 2004; Clemens et al., 2005) and nutritional deprivation (Youdim et al., 1981). One of the main criteria of RLS is an urge to move (Murray et al., 1988; Ondo et al., 2007). This symptom is significantly more difficult to simulate in an animal model due to no subjective feedback. More importantly, a greater focus should be employed in obtaining sensory data from potential animal models of RLS (see Table 1.1) and establishing novel methods for testing these sensory differences in-vivo.

Currently, in light of the different symptomatology and criteria, the pursuit of an ideal model is still far. When a potential unified model displaying all symptoms has been produced, a series of different tests will need to be implemented to validate whether these symptoms match those described in human RLS. Obviously, efforts must be focused on generating a unified model, however particular attention must be made on deciding the group of tests best suited for use when showing the desired phenotype in a particular animal. Animal choice is 
also important, a majority of experiments have been performed using rats, however the whole genome is undetermined for these animals and thus a lack of genetic models are available. Although, the lower level of intelligence of mice in comparison to rats is a counterbalancing argument. In addition, inducing iron-deficiency in animals is well established for over thirty years (Dallman et al., 1975) and the pathophysiological effects are well defined, therefore, using animal models of iron-deficiency to research the importance of iron as a causative factor of RLS seems logical due to the current wealth of knowledge in this field.

\subsubsection{Importance for research}

Due to the increasing prevalence, the associated unpleasant symptoms and the nature of RLS in affecting people of a wide-age range, suggests the importance of continuing research in this field. In addition, research in iron-deficiency will be of great medical use due to the high prevalence of iron-deficiency worldwide and its implications in many neurological conditions, namely Alzheimer's and Huntington's diseases (Youdim et al., 1993; Gerlach et al., 1994; Faucheux et al., 1995; Loeffler et al., 1995; Ponting, 2001; Bishop et al., 2002). Currently, a large wealth of clinical knowledge exists for RLS, however there is a shortage of detailed scientific studies in this field. For example the lack of understanding in how certain causative factors induces symptoms. Therefore, a solution to this problem is to design a suitable mammalian animal model with either genetic or phenotypic similarities to human RLS which will help investigate the causative factors of RLS and allow a more detailed observation of them in-vivo. However, this task has still not been fully achieved and remains a necessity. Furthermore, such a model will aid pharmaceutical companies in trialing the benefits of novel compounds prior to clinical phase I.

\subsection{Aims and objectives of this thesis}

The initial aim is to establish the effects of acute and chronic administered iron deprived diet upon the general physiology of C57B1/6 wildtype (WT) male mice. In particular observations will be made into whether the diet is producing a controlled level of iron-deficiency and not inducing any unwanted anaemic effect. The three main criteria for designing a suitable animal model; sensory, motor activity and circadian onset of motor activity, will be investigated in WT and dopamine receptor 3 null (D3 ${ }^{--}$) male mice by means of using different behavioural tests to evaluate each criterion. Differences in sensory behaviour will be measured by both hot-plate and formalin tests, whereby the effects of iron-deficiency upon motor activity and its circadian onset, will be assessed by means of the voluntary wheel-running test. In parallel to the behavioural analysis, immunohistochemical assessment of c-Fos and ChAT will be used to 
identify any sensory and motor induced cellular changes occurring at the lumbar dorsal horn and thoracolumbar ventral motoneurons of the CPG region, respectively. Finally, screening for dysregulated proteins in the midbrain region from ID WT compared to control WT mice will utilise two dimension fluorescence difference in gel electrophoresis (2-D DIGE), where matrix-assisted laser desorption/ionisation time-of-flight mass spectrometry (MALDI-TOFMS) will be essential in determining potential novel protein targets altered in-vivo during irondeficiency. 
Chapter 2 Results 


\section{Results}

\subsection{Controlling iron consumption in the mouse's environment}

Before any experiments were performed, the environment in which mice were maintained in was controlled. This meant every component in mouse's cage was analysed for iron content. Furthermore, this ensured that the low concentration $(<8 \mathrm{mg} \mathrm{Fe} / \mathrm{kg}$ feed $)$ of iron within the specially formulated ID diet for mice was the only source from which iron could be obtained.

Initially, the level of iron within the water supply was tested from three independent samples $(n=3)$ in addition to the bedding and all other components of the cage. Based on the detected iron concentration within the different water samples (see below Table 2.1), meant all mice were maintained on tap water, due to the similar iron concentrations between tap, deionised and double distilled water $\left(\mathrm{ddH}_{2} \mathrm{O}\right)$ sources. Other components of the cage, including bedding and the metal bar which was used to separate the bedding from the running wheel contained negligible concentrations of iron.

\begin{tabular}{|c|c|}
\hline Source & $\begin{array}{c}\text { Iron-concentration }(\boldsymbol{\mu M} / \mathbf{L}) \\
( \pm \text { SEM) }\end{array}$ \\
\hline $\mathrm{ddH}_{2} \mathrm{O}$ & $0.03( \pm 0.03)$ \\
\hline de-ionised water & $0.03( \pm 0.03)$ \\
\hline tap water in animal house & $0.03( \pm 0.03)$ \\
\hline automatic water source & $0.10( \pm 0.06)$ \\
\hline wood cuttings (bedding) & $0.06( \pm 0.03)$ \\
\hline metal bar & $0.03( \pm 0.03)$ \\
\hline
\end{tabular}

Table 2.1. Iron concentration of different components in the mouse's environment. The iron concentration was measured from three independent samples $(n=3)$ of water, bedding and the metal bar used only for wheelrunning experiments to separate the bedding from the wheel. Values given in $\mu \mathrm{M} / \mathrm{L}( \pm \mathrm{SEM})$.

\subsection{Evaluating the effect of ID diet upon the general physiology}

Monitoring the mouse's weight proved important for determining the general health of the animal. Furthermore, the weight helped determine the earliest duration of ID diet having an effect in the mouse. The mice which were used for weight and blood analysis were "static" within the cage, i.e. no wheel-running activity. These mice had their weights' monitored every week, up to a maximum duration of 30 weeks after commencing either the control or ID diets at post-natal day 28 (P28). Described in the methods section 4.1, all animals were grouped at P28, where the final group weight average was identical between control and ID animals.

After 1 week of ID diet, a decrease in weight was observed between control $(17.9 \mathrm{~g}$ $\pm 0.614)$ and ID $(16.5 \mathrm{~g} \pm 0.641)$ mice (see Fig. 2.1a), however a post hoc test showed the groups to be not significantly different $(P>0.05)$. Moreover, 2, 3 and 4 weeks of ID diet resulted in the largest early differences between control and ID animals, where post hoc tests 
revealed the average weight values to be significantly reduced $(P<0.05)$ in WT ID compared to control mice, as shown in Fig. 2.1a. The only time points which showed a lack of significance $(P>0.05)$ were at $1,8,10,11,17,26-30$ weeks (see Fig. 2.1a). An ANOVA test for repeated measures showed main effects of diet and the interaction of duration (weeks) and diet upon the average group weight $(P<0.05$; Table 2.2).

Haematology analysis involved measuring the plasma-iron concentration, haemoglobin and haematocrit from the blood of both control and ID WT mice at varying stages of diet administration, starting from P28 (0 weeks) and continuing up to 30 weeks. Haemoglobin is an iron-containing metalloprotein found in erythrocytes and is involved in oxygen transport within the blood, where haematocrit is a measure of the packed cell volume of erythrocytes. Both these parameters in addition to plasma-iron are standardised and established measurements when assessing the effects an ID diet has upon the iron content/transporting capacity in mouse blood (Beard et al., 2005).

Measuring these parameters proved important when assessing if the ID diet compared to the normal diet for controls, was having an effect upon the iron levels in mice. Moreover, these experiments provide the basic knowledge from which subsequent behavioural/cellular/protein experiments will derive from. Interestingly, the initial change observed in all three blood parameters was observed after 1 week of diet (Fig. 2.1b-d). Post hoc tests confirm this by showing plasma-iron concentration, haematocrit and haemoglobin to be significantly reduced $(P<0.05)$ compared to control mice. The ANOVA showed a main effect of duration as well as diet upon plasma-iron, haemoglobin and haematocrit $(P<0.05$; Table 2.2). Moreover, the interaction of diet and duration was significant $(P<0.05$; Table 2.2) for haemoglobin and haematocrit, although not significant for plasma-iron $(P<0.05$; Table 2.2). Also, an initial decrease (27\%) in the plasma-iron concentration was found after 1 week of ID diet, where at every time-point, except 0 weeks, the post hoc tests revealed a significant $(P<0.05)$ decrease in plasma-iron concentration between control and ID mice. After this initial change in plasma-iron after 1 week of ID diet, a steady concentration of low iron in the plasma was reached after 4 weeks $(15.3 \mu \mathrm{M} / \mathrm{L} \pm 2.0)$ continuing through to 30 weeks (16.3 $\mu \mathrm{M} / \mathrm{L} \pm 2.6)$ (Fig. 2.1b), where no significant difference $(P>0.05)$ in the plasma-iron level between 4 and 30 weeks was shown for ID mice. In addition, the plasma-iron level of control/ID $\mathrm{D}^{-/}$mice $(32.6 \mu \mathrm{M} / \mathrm{L} \pm 2.1 / 20.4 \mu \mathrm{M} / \mathrm{L} \pm 1.7)$ after 15 weeks ID diet was significantly reduced $(P<0.05)$, where the level of decrease was equivalent $(P>0.05)$ to that seen for WT mice after 15 weeks ID diet. The grey area contained within the red lines as shown in Fig. 2.1c-d, represent the normal range (upper and lower limits) of haemoglobin and haematocrit. This data was acquired from Zentralen tierexperimentellen Einrichtungen (ZTE) 
Göttingen and represents the range corresponding to the WT mouse strain. In addition, the haematocrit levels are in the normal range as previously described (Dean et al., 2006).

At 15 weeks of diet P28, forelimb grip strength was measured to detect any change in neuromuscular integrity and muscle strength which may occur after prolonged iron-deficiency. In previous studies grip strength was investigated in offspring from ID mothers (dams) administered an iron reduced diet (12.5-14 $\mu \mathrm{g}$ Fe/g diet) (Kwik-Uribe et al., 1999; Kwik-Uribe et al., 2000b), however this has not been investigated in mice from control dams which are administered an ID diet at post-natal stages. Therefore, this would act as a good indicator in determining whether the ID diet is inducing any change in muscle strength in these mice, and if the case may later influence further behavioural testing, for example the wheel-running test. Post hoc tests showed no significant difference $(P>0.05)$ in the grip force between control $(n=15)$ and ID mice $(n=15)$ at 15 weeks of diet administration P28. A similar result showing no significant difference $(P>0.05)$ in forelimb grip strength was identified between ID and control mice after 15 weeks of wheel-running activity.
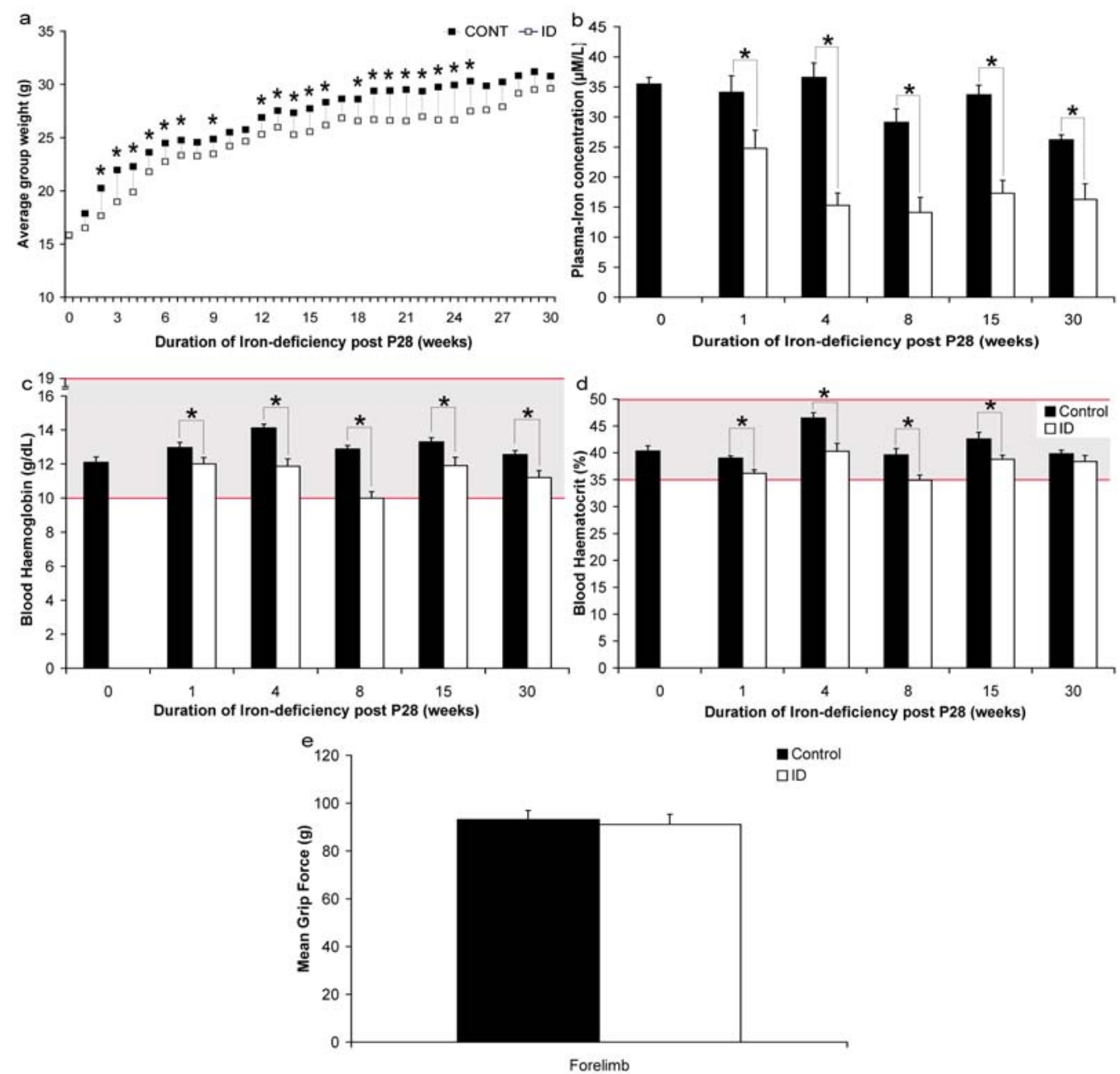

Fig. 2.1. Effect of ID diet upon general physiology. a. Weight was recorded continuously over a 30 week period for control ( $\mathbf{\square})$ and ID ( $\square$ ) mice. Three blood parameters were measured for control ( \pm SEM, $n=7)$ and ID $(\square \pm$ SEM, $n=7)$ mice maintained on different durations of control or ID diet post P28. These were, b. Plasma-iron $(\mu \mathrm{M} / \mathrm{L})$, c. Haemoglobin $(\mathrm{g} / \mathrm{dL})$ and d. Haematocrit (\%). e. Forelimb grip strength, mean grip force (g) was also measured for both control $(\square \pm$ SEM, $n=15)$ and ID ( $\square \pm$ SEM, $n=15)$ mice. Significant differences $(P<0.05)$ are marked by an asterisk. 


\subsection{Assessing sensory behavioural differences}

\subsubsection{The hot-plate test}

\subsubsection{Iron-deficiency reduces hot-plate reaction time in WT mice}

This study evaluated whether iron-deficiency modifies the acute pain threshold, and whether the duration of iron-deficiency is a participating factor.

The hot-plate behavioural test was used to determine the acute sensory response differences between control and ID mice. Under controlled hot-plate temperature settings $\left(50^{\circ} \mathrm{C} \pm 0.5\right)$, the elapsed time from placement upon the hot-plate until the mouse jumped or the first hindpaw licking, was denoted as the reaction time in seconds. The ANOVA showed a main effect of duration as well of diet upon the hot-plate response score (Time) $(P<0.05$; Table 2.2). Post hoc tests revealed that the reaction time for the ID group was significantly reduced $(P<0.05)$ in both the 4 and 15 week groups as compared to the controls (see Fig. 2.2a). However, no significant difference was observed after 1 week of ID diet. Overall, it was found that ID diet reduces the hot-plate reaction time in mice only at 4 and 15 week durations of diet P28.

\subsubsection{Reduced hot-plate reaction time in $D 3^{-/-}$mice}

The second experiment showed whether the presence of the D3 receptor altered the reaction time and whether iron-deficiency was an influencing factor. This was performed on $\mathrm{D}^{-/-}$mice at 15 weeks diet duration P28.

The hot-plate behavioural test was used to determine the acute sensory response differences between control and ID mice for the WT and D3 $3^{-/}$strains. A main effect of strain as well as diet upon the hot-plate response score (Time) $(P<0.05$; Table 2.2$)$ was shown by the ANOVA. However, the interaction of diet and strain was not significant $(P>0.05$; Table 2.2). Under controlled hot-plate temperature settings $(P>0.05)$, post hoc tests revealed that the reaction time for the ID group was significantly reduced $(P<0.05)$ compared to the controls (see Fig. 2.2b), however for the $\mathrm{D} 3^{-/-}$strain there was no significant difference $(P>$ $0.05)$ in reaction time between ID and control groups. Despite this, post hoc tests revealed D3-

/- ID and control mice to have a significantly quicker $(P<0.05)$ hot-plate reaction time compared to the corresponding WT diet groups. The results of the current hot-plate study have further confirmed our previous findings that ID diet significantly reduces the reaction time at 15 weeks diet P28 in WT mice (see Fig. 2.2). In addition, no synergistic decrease was observed between control and ID D3 ${ }^{-/-}$groups. 

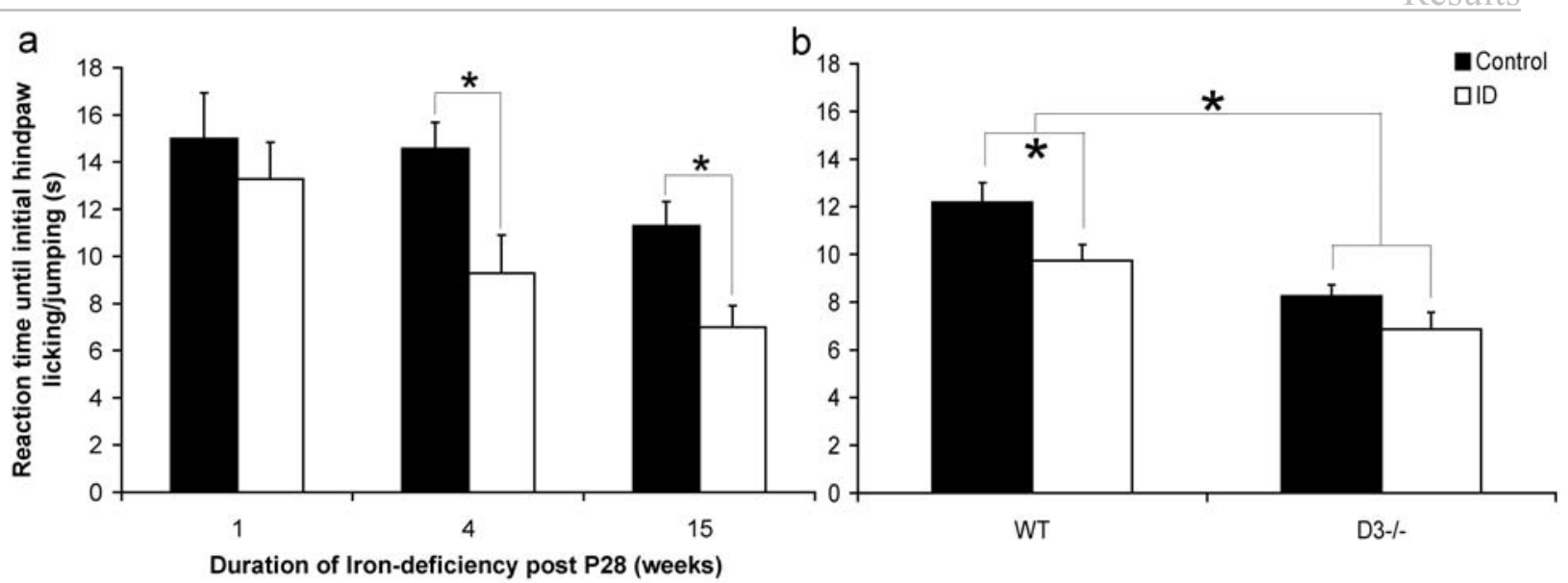

Fig. 2.2. Effect of ID diet upon hot-plate reaction time. a. Different hot-plate reaction times (seconds) of both control ( $\square$ SEM, $n=7)$ and ID ( $\square \pm$ SEM, $n=7)$ WT mice at 1,4 and 15 week diet durations P28. b. A different experiment shows the influence a lack of D3 receptors (D3-/-) has upon the acute pain response time, which is shown between WT and D3-/- mice at a 15 week diet duration P28, administered either a control $(\boldsymbol{\square} \pm \mathrm{SEM}, n=15)$ or ID ( $\square \pm \mathrm{SEM}, n=15)$ diet. Significant differences $(P<0.05)$ are marked by an asterisk.

\subsubsection{The formalin-test}

\subsubsection{Iron-deficiency elevates inflammatory pain response in WT mice}

The formalin-test was assessed in both control and ID WT mice for the different groups, 1 week (Fig. 2.3b), 4 weeks (Fig. 2.3c) and 15 weeks (Fig. 2.3d). This test was used to determine the pain response difference induced by a prolonged inflammatory mediated pain via C-fibres, which was exhibited during the second phase (phase II) of the test. Therefore, compared to the hot-plate test a different primary afferent fibre was assessed.

ANOVA for repeated measures showed main effects of duration, diet and time upon the response score of the formalin-test $(P<0.05$; Table 2.2). All the interactions were significant apart from duration and diet, as well as duration, diet and time $(P>0.05$; Table 2.2). Post hoc tests showed the ID group to have an increased pain response $(P<0.05)$ compared to controls during phase II of the formalin-test at all tested durations of diet $(1,4$ and 15 weeks). An external measure was implemented ensuring the pain inducing stimulus from the formalin injection was controlled between groups. No difference $(P>0.05)$ in the level of inflammation (inflammation score) was observed between control and ID groups at weeks 1, 4 and 15 (Fig. 2.3a).

\subsubsection{Iron-deficiency synergistically elevates the inflammatory pain response in $D 3^{-/}$mice}

The D3 receptor has a suggested action in RLS. Therefore, investigating the pain response of $\mathrm{D}^{-/-}$mice may give insight into its function in modulating the pain response and its interaction with iron-deficiency.

The formalin-test was assessed in both control and ID mice for the different strains, WT and $\mathrm{D}^{-/-}$(Fig. 2.3f). ANOVA for repeated measures showed main effects of strain, diet 
and time upon the response score of the formalin-test $(P<0.05$; Table 2.2). Also the interactions time and diet, time and strain were significant $(P<0.05$; Table 2.2), however the following interactions were not; strain and diet, as well as strain, diet and time $(P>0.05$; Table 2.2). Post hoc tests revealed WT and $\mathrm{D}^{-/-}$ID groups to have an increased pain response $(P<0.05)$ compared to controls during phase II of the formalin-test $(30-45$ minutes $)$, where a synergistic increase in formalin-induced pain prevails in $\mathrm{D} 3^{-/-}$ID mice. Moreover, a similar pain profile between WT ID and D3 $3^{-/}$control mice was shown during phase II. An external measure was implemented ensuring the pain inducing stimulus was controlled. No difference $(P>0.05)$ in the level of inflammation (inflammation score) was observed between control and ID groups for the WT and D3 ${ }^{-/-}$strains (Fig. 2.3e).
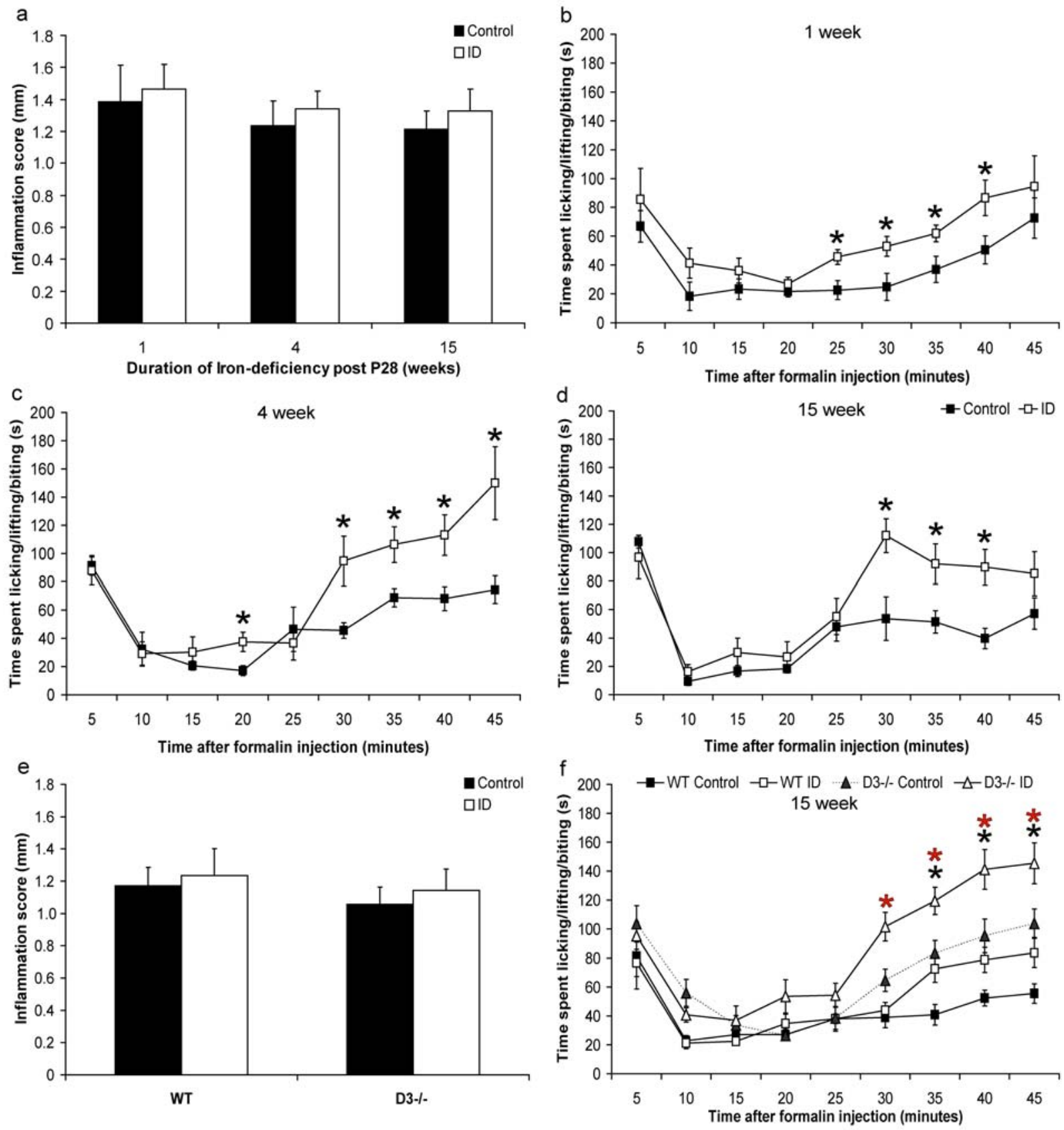
Fig. 2.3. The effect of ID diet on formalin-induced pain. a, e. The degree of inflammation after injecting formalin into the hindpaw was scored by using callipers. The difference (ipsilateral hindpaw thickness contralateral hindpaw thickness) was measured (mm) between control $(\boldsymbol{\square} \pm \mathrm{SEM}, n=7)$ and ID mice ( $\square \pm \mathrm{SEM}, n=7)$ at the different test stages which correspond to the different durations of iron-deficiency P28. After formalin injection, WT control ( $\square$ SEM, $n=7)$ and WT ID mice ( $\square \pm \mathrm{SEM}, n=7)$ were monitored for 45 minutes, where after every 5 minutes the total time (seconds) spent licking/lifting/biting the injected hindpaw was recorded. The formalin-tests were completed for the varying durations (weeks) of iron-deficiency P28, b. 1 week, c. 4 week and d. 15 week. f. The formalin test on $\mathrm{WT}$ and $\mathrm{D} 3^{-/}$mice at the diet duration of 15 week. Time-points showing significant differences $(P<0.05)$ between control and ID WT mice are marked by a black asterisk, where a red asterisk shows differences between control and ID D3 $3^{-/-}$mice.

\subsubsection{Heightened central cellular activity detected by ipsilateral c-Fos expression in ID WT mice}

c-Fos expression in laminae I/II of the ipsilateral and contralateral dorsal horns were quantified in control and ID mice from the 1, 4 and 15 week groups. The ANOVA showed main effects of duration, diet as well as dorsal horn side (ipsilateral or contralateral) upon the number of c-Fos immunoreactive (c-Fos-IR) cells present in the ipsilateral dorsal horn $(P<$ 0.05; Table 2.2). In addition, the interactions of duration and dorsal horn side as well as diet and dorsal horn side were significant $(P<0.05$; Table 2.2$)$. Generally, as a response to the formalin injection a strong expression of c-Fos-IR cell nuclei were clearly observed at the superficial laminae of the ipsilateral dorsal horn in all mice, as shown at the immunofluorescence (Fig. 2.4a) and immunoperoxidase (Fig. 2.4b-e) levels. In contrast, the contralateral horn shows relatively low levels of c-Fos expression (Fig. 2.4a and Fig. 2.4g). Post hoc tests between ID and control groups at 1 and 4 weeks showed a significant increase $(P<0.05)$ in number of c-Fos-IR cells at the ipsilateral horn from ID WT mice. 

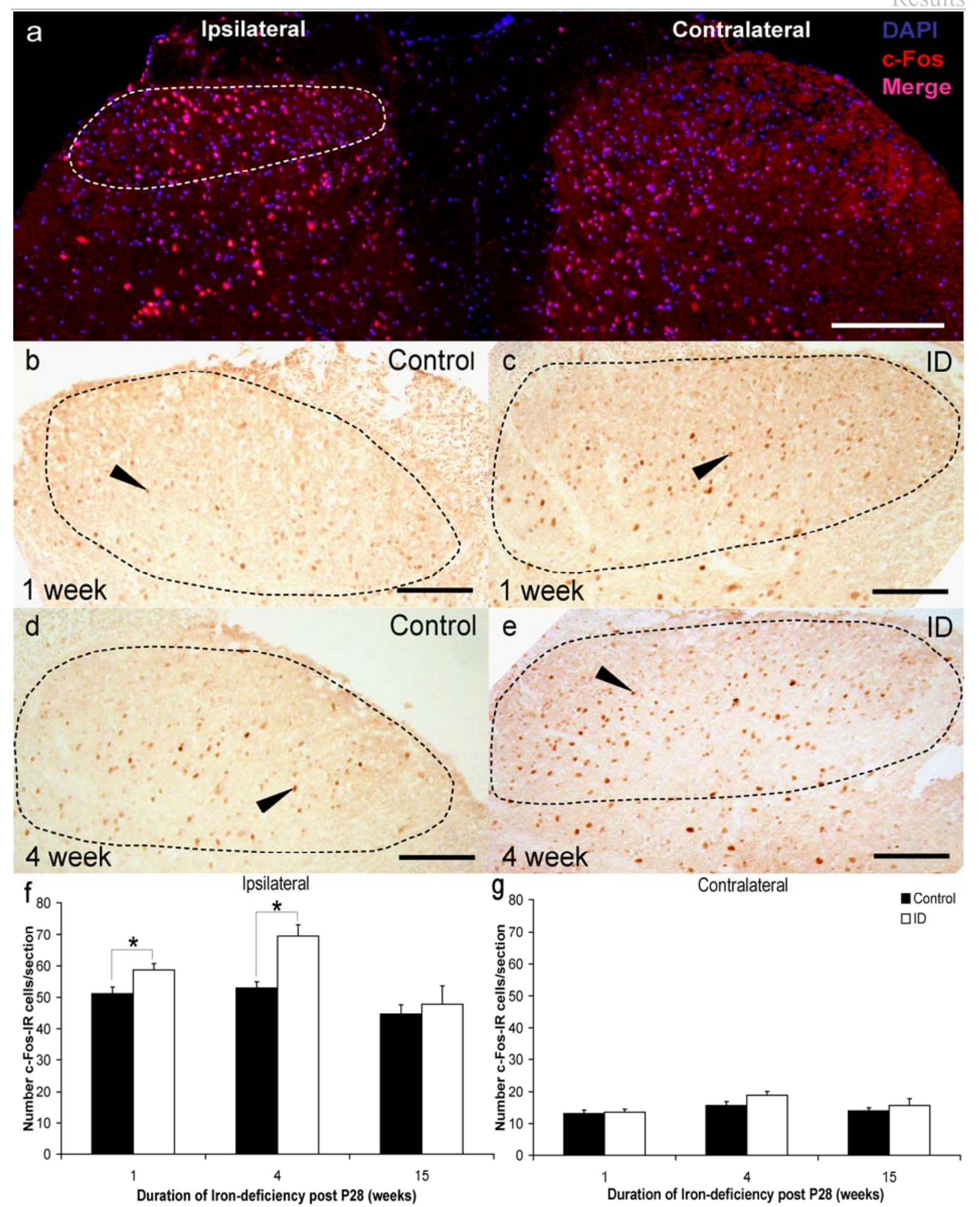

Fig. 2.4. The effect of different durations of ID diet in WT mice upon formalin-induced c-Fos expression. a. After formalin injection into the left hindpaw, elevated c-Fos immunoreactivity results, as shown at laminae I/II of the ipsilateral dorsal horn (enclosed within a dashed line) compared to the contralateral horn, in an ID mouse after 1 week iron-deficiency P28. The c-Fos expression labelled by indocarbocyanine 3 (Cy3) (red)-conjugated secondary antibody, in conjugation with 4',6-diamidino-2-phenylindole (DAPI) (blue) shows co-localisation producing a merged pink colour easily identifiable at superficial laminae I/II. Scale bar represents $200 \mu \mathrm{m}$. b-e. cFos immunoreactivity in the ipsilateral horn labelled by an immunoperoxidase method, as indicated by the black arrow heads, corresponds to laminae I/II, as shown in a. Differences between control and ID mice were seen at both a 1 week (b and $\mathbf{c}$ ) and 4 week (d and e) of iron-deficiency P28. Scale bar represents 100 $\mu \mathrm{m}$. The number of c-Fos-IR cells found within laminae I/II of the $\mathbf{f}$. ipsilateral and $\mathbf{g}$. contralateral horns, were quantified for all three durations of iron-deficiency P28, where differences in the average number of c-Fos-IR cells/section are illustrated for both control $(\square \pm \mathrm{SEM}, n=7)$ and ID mice ( $\square \pm \mathrm{SEM}, n=7)$. Significant differences $(P<0.05)$ are marked by an asterisk. 
Table 2.2

Results of the ANOVAs

\section{Forelimb Grip Strength}

Diet

Weight

Diet

Duration $\times$ Diet

Duration $\times$ Diet

Plasma-iron

\section{Duration}

Diet

Duration $\times$ Diet

\section{Hot-plate test BI/6}

\section{Duration}

Diet

Duration $\times$ Diet

\section{Hot-plate test D3-\%-}

\section{Diet}

Strain

Strain $\times$ Diet

${ }^{*} P<0.05$.

\begin{tabular}{|c|c|c|c|c|c|c|}
\hline \multirow{3}{*}{$\begin{array}{c}\text { Degrees of freedom } \\
1\end{array}$} & & & Formalin-test BI/6 & \multirow[b]{2}{*}{ Degrees of freedom } & \multirow[b]{2}{*}{$F$ values } & \multirow[b]{2}{*}{$P$ values } \\
\hline & $F$ values & $P$ values & & & & \\
\hline & 0.115 & 0.737 & Time & 8 & 41.349 & $<0.001^{*}$ \\
\hline & & & Duration & 2 & 4.341 & $0.020^{*}$ \\
\hline & & & Diet & 1 & 28.346 & $<0.001^{*}$ \\
\hline 1 & 5.974 & $0.023^{*}$ & Time $\times$ Duration & 16 & 3.160 & $<0.001^{*}$ \\
\hline \multirow[t]{2}{*}{30} & 2.145 & $<0.001 *$ & Duration $\times$ Diet & 2 & 0.041 & 0.960 \\
\hline & & & Time $\times$ Duration $\times$ Diet & 16 & 1.137 & 0.320 \\
\hline 5 & 21.066 & $<0.001^{*}$ & & Degrees of freedom & $F$ values & $P$ values \\
\hline 1 & 36.843 & $<0.001^{*}$ & Time & 8 & 54.456 & $<0.001^{*}$ \\
\hline \multirow[t]{3}{*}{4} & 5.546 & $0.001^{*}$ & Diet & 1 & 7.052 & $0.014^{*}$ \\
\hline & & & Strain & 1 & 27.839 & $<0.001^{*}$ \\
\hline & & & Time $\times$ Diet & 8 & 5.766 & $<0.001^{*}$ \\
\hline Degrees of freedom & $F$ values & $P$ values & Time $\times$ Strain & 8 & 6.572 & $<0.001^{*}$ \\
\hline 5 & 10.240 & $<0.001^{*}$ & Strain $\times$ Diet & 1 & 0.863 & 0.362 \\
\hline Degrees of freedom & $F$ values & $P$ values & Duration & 2 & 13.437 & $<0.001^{*}$ \\
\hline 5 & 3.829 & $0.004^{*}$ & Diet & 1 & 16.446 & $<0.001^{*}$ \\
\hline 1 & 93.699 & $<0.001^{*}$ & Dorsal Horn Side & 1 & 840.382 & $<0.001^{*}$ \\
\hline \multirow[t]{3}{*}{4} & 2.286 & 0.068 & Duration $\times$ Diet & 2 & 2.837 & 0.059 \\
\hline & & & Duration $\times$ Dorsal Horn Side & 2 & 7.421 & $0.001^{*}$ \\
\hline & & & Diet x Dorsal Horn Side & 1 & 7.506 & $0.006^{*}$ \\
\hline Degrees of freedom & $F$ values & $P$ values & Duration $\times$ Diet $\times$ Dorsal Horn Side & 2 & 1.538 & 0.216 \\
\hline 2 & 6.327 & $0.004^{*}$ & & & & \\
\hline 1 & 10.699 & $0.002^{*}$ & & & & \\
\hline 2 & 0.855 & 0.434 & & & & \\
\hline Degrees of freedom & $F$ values & $P$ values & & & & \\
\hline
\end{tabular}




\subsection{Assessing motor behavioural differences}

\subsubsection{The voluntary wheel-running test}

Two distinct activity periods are observed when studying the 24 hour circadian behaviour of mice. Firstly, mice are nocturnal therefore during the 12 hour dark phase (19:00hr-07:00hr) an elevated activity is observed compared to the quiescent activity during the 12 hour light phase (07:00hr-19:00hr). This result section will primarily focus on highlighting the wheel-running behaviour during the active dark phase and at periods comparable to when RLS symptoms prevail in humans. The parameters obtained from using a voluntary wheel-running system are each very distinct and individually important. Certain parameters will be termed performance and others activity.

\subsubsection{Wheel-running performance in WT mice}

The voluntary wheel-running behaviour was assessed in both control and ID WT mice (Fig. 2.5) at two different durations of ID diet, 4 weeks (Fig. 2.5a,c,e) and 15 weeks (Fig. 2.5b,d,f). The parameters used to assess the mouse's performance on the wheel and whether it was influenced by an ID diet were, distance ran in metres (Distance (m)), maximum running speed in metres/second $\left(\mathrm{V}_{\max }(\mathrm{m} / \mathrm{s})\right)$ and finally the average distance per run (Distance $/ \mathrm{N}_{\text {run }}(\mathrm{m} / \mathrm{run})$ ). At both 4 and 15 week durations, no influence of diet upon the performance was observed between ID and control mice (Fig. 2.5) during the dark phase. This was demonstrated by an ANOVA where no effect of diet, duration and time upon Distance, $\mathrm{V}_{\max }$, and Distance $/ \mathrm{N}_{\text {run }}(P$ $>0.05$; Table 2.3) was observed. Therefore, after the 15 week duration of diet no observable variation in the performance was seen when compared to the 4 week diet duration, with the exception of Distance during the beginning period of the dark phase, where a slight yet significant decrease $(P<0.05)$ in this parameter was shown in ID WT mice after 15 weeks of diet (Fig. 2.5b). Despite this, a lack of significance was shown in the interaction between diet and duration for Distance, $\mathrm{V}_{\max }$, and Distance $/ \mathrm{N}_{\mathrm{run}}(P>0.05$; Table 2.3). 

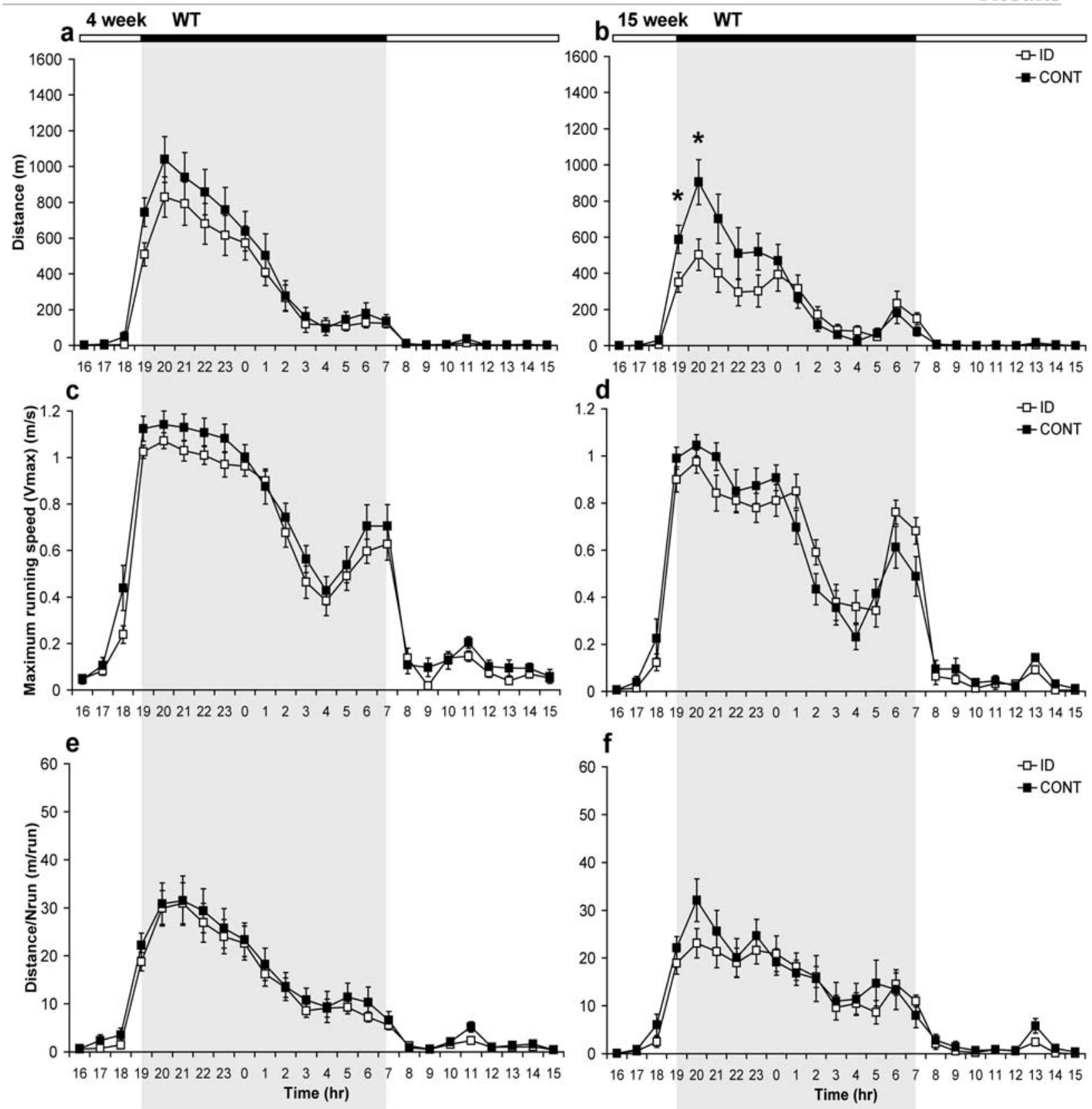

Fig. 2.5. Influence of ID diet and duration upon voluntary wheel-running performance in WT mice. a,c,e. Wheel-running activity is presented for ID ( $\square \pm \mathrm{SEM}, n=15)$ and control $(\boldsymbol{\square} \pm \mathrm{SEM}, n=12)$ mice at a diet duration of 4 weeks P28, and $\mathbf{b}$,d,f. for ID ( $\square \pm$ SEM, $n=14)$ and control $(\square \pm$ SEM, $n=11)$ mice at a diet duration of 15 weeks P28. Three different parameters were recorded at the two diet durations to assess the effect ID diet has upon the wheel-running performance over a 24 hour time period (hr), as shown by the $\mathrm{x}$-axis, these were a,b. Total Distance ran (metres) for every hour, c,d. Maximum running speed (metres/second) and e,f. Average distance per run (metres/run). Compared to the light phase (07:00hr-19:00hr), the nocturnal nature of mice show elevated running performance during the dark phase $(19: 00 \mathrm{hr}-07: 00 \mathrm{hr})$, represented by the grey region projected from the black portion of the overhanging bar $(\mathbf{a}, \mathbf{b})$.

\subsubsection{Iron-deficiency increases wheel-running activity in WT mice}

The remaining four parameters are concerned with the mouse's activity rather than performance, as previously shown (Fig. 2.5). The first two parameters show the number of runs $\left(\mathrm{N}_{\mathrm{run}}\right)$ (Fig. 2.6a,b) and percentage of the maximum number of runs at each time-point (Percentage of $\max \mathrm{N}_{\text {run }}$ (\%)) (Fig. 2.6c,d), for both 4 (Fig. 2.6a,c) and 15 (Fig. 2.6b,d) week diet durations. The second set of parameters are the total activity time spent running $\left(\mathrm{T}_{\text {run }}\right.$ 
(minutes)) (Fig. 2.7a,b) and the percentage of the maximum activity time spent running (Percentage of max $\mathrm{T}_{\text {run }}(\%)$ ) (Fig. 2.7c,d), similarly at both 4 (Fig. 2.6a,c) and 15 (Fig. 2.6b,d) week diet durations. The percentage parameters represent relative values based from the former absolute values $\left(\mathrm{N}_{\text {run }}\right.$ and $\left.\mathrm{T}_{\text {run }}\right)$. The reason for creating these relative values becomes apparent when noticing the group mean $\mathrm{N}_{\text {run }}$ and $\mathrm{T}_{\text {run }}$ values for ID WT mice during the 2 hour period (06:00hr-07:00hr) prior to lights on and how the group values are virtually identical to the maximum $\mathrm{N}_{\text {run }}$ or $\mathrm{T}_{\text {run }}$ values seen at the start of the dark phase (19:00hr-21:00hr), infact there is no significant difference $(P>0.05)$ between these two periods. Therefore, the reasons why ID WT mice sustain this heightened level of activity at this time-point are of particular interest and will be discussed later.

By observing the running activity of ID mice at 15 weeks diet duration, a unique running profile can be seen at the 06:00hr-07:00hr time period, i.e. 2 hours prior to the start of the light phase. This period is highlighted within the red box as shown in Fig. 2.6b,d and $2.8 \mathrm{~b}$, d. Interestingly, for all activity parameters during the dark phase, the interaction of time and diet is significantly different at only the 15 week diet period $(P<0.05$; Table 2.4), therefore no difference was noted at the 4 week diet period $(P>0.05$; Table 2.4). Post hoc tests revealed the following parameters, Percentage of max $\mathrm{N}_{\text {run }}$ (Fig. 2.6f) and Percentage of $\max \mathrm{T}_{\text {run }}$ (Fig. 2.7f), at this time-period $(06: 00 \mathrm{hr}-07: 00 \mathrm{hr}$ ) to be significantly increased by approximately 2 fold in ID WT mice $(P<0.05)$ compared to control WT mice, where this significant difference was not present $(P>0.05)$ during the 4 week diet duration (Fig. 2.6e and 2.8e). In addition, further post hoc analysis of $\mathrm{T}_{\text {run }}$ for the 15 week diet group, revealed a significant $(P<0.05)$ increase in activity time for only ID WT mice compared to control WT at $07: 00 \mathrm{hr}$ (Fig. 2.7b). Overall, the main effect of an elevated activity observed prior to the start of the light phase, occurs after 15 weeks of ID diet. Moreover, this period prior to the start of the light phase for mice is equivalent to the late evening and prior to sleep in humans. Furthermore, duration had an effect on all activity parameters during the dark phase, $\mathrm{N}_{\text {run }}$, Percentage of max $\mathrm{N}_{\text {run }}, \mathrm{T}_{\text {run }}$ and Percentage of $\max \mathrm{T}_{\text {run }}(P<0.05$; Table 2.4).

Despite ID WT mice showing this heightened level of activity at 06:00hr-07:00hr, they appear to show a reduced activity compared to control WT mice during the initial part of the dark phase as shown by $\mathrm{N}_{\text {run }}$ (Fig. 2.6a,b) at both 4 and 15 weeks of diet. However, the presence of any change during these initial stages of the dark phase was not observed for Percentage of max $\mathrm{N}_{\text {run }}$ at both 4 and 15 weeks diet (Fig. 2.6c,d). Regarding $\mathrm{T}_{\text {run }}$, a significant decrease $(P<0.05)$ in activity time of ID WT mice was also shown at the beginning of the dark phase after 4 and 15 weeks of diet (Fig. 2.7a,b). This was further indicated by the relative value Percentage of max $T_{\text {run }}$ (Fig. 2.7c,d). 

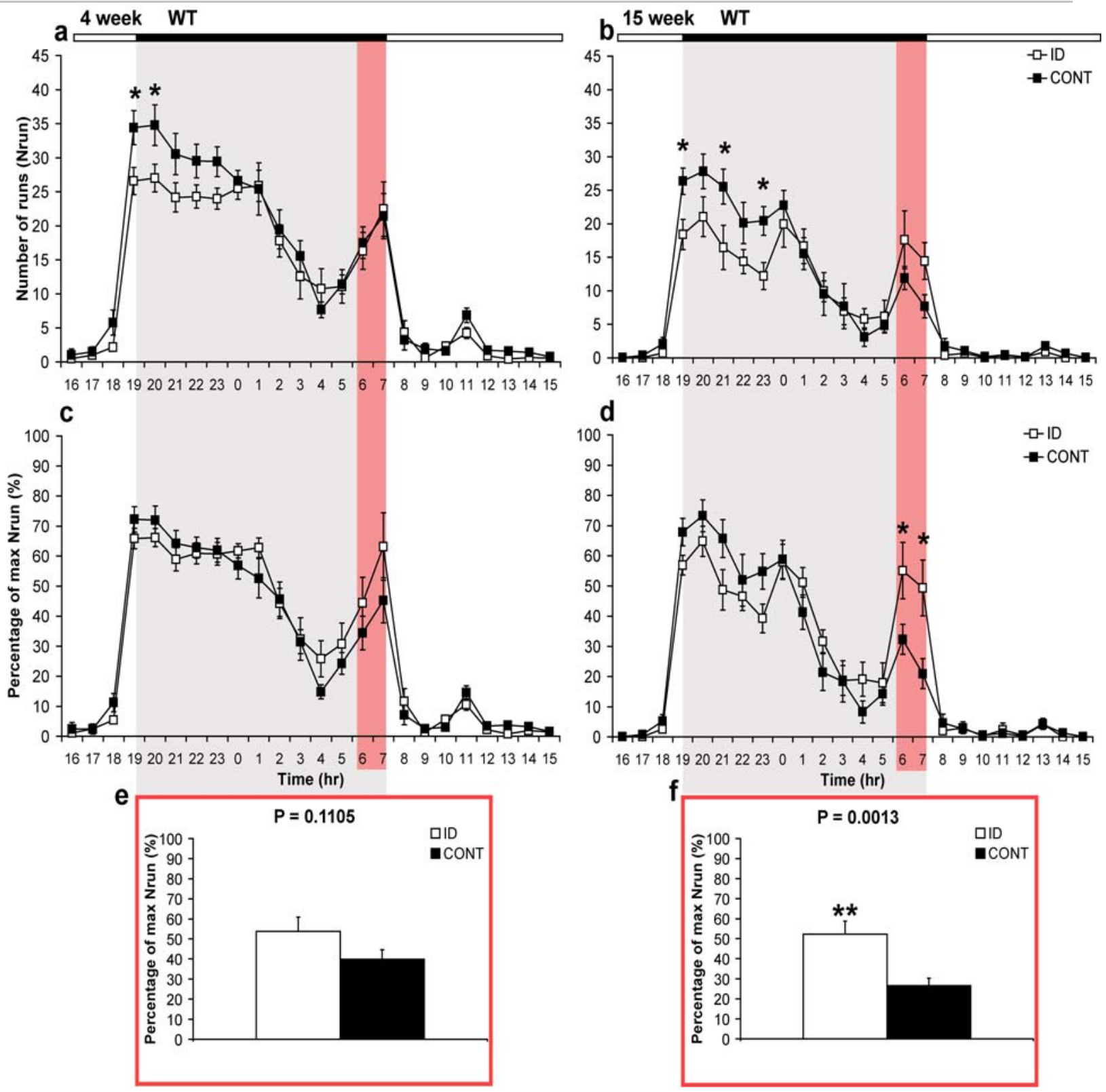

Fig. 2.6. ID diet alters the activity shown by number of runs during the late phase of the active cycle in WT mice. a,c,e. Wheel-running activity is presented for ID ( $\square \pm \mathrm{SEM}, n=15)$ and control $(\mathbf{\square} \pm \mathrm{SEM}, n=12)$ mice at a diet duration of 4 weeks P28, and $\mathbf{b , d , f}$. for ID ( $\square \pm \mathrm{SEM}, n=14)$ and control $(\mathbf{\square} \pm \mathrm{SEM}, n=11)$ mice at a diet duration of 15 weeks P28. The parameter shown, $\mathbf{a}, \mathbf{b}$. describes the number of times the mice voluntarily ran $\left(\mathrm{N}_{\text {run }}\right)$ on the running wheel at the two diet durations over a 24 hour time period (hr), as shown by the x-axis. From these absolute values (a,b.) relative values (c,d.) were calculated for every mouse independently c,d. showing the percentage of runs, which is the number of runs the mouse ran in the hour when compared to the maximum recorded number of runs it achieved during the period 19:00hr-03:00hr. Compared to the light phase (07:00hr-19:00hr), the nocturnal nature of mice show elevated running activity during the dark phase (19:00hr07:00hr), represented by the grey region projected from the black portion of the overhanging bar (a,b). The red highlighted portion of the grey region represents the final 2 hour period prior to the start of the light cycle (07:00) i.e. before mice rest. Values highlighted red, originating from c,d. are represented in the graphs within the corresponding red boxes, e,f. showing the difference $(P<0.05)$ ID diet has upon activity during the final 2 hour period prior to lights on. Significant differences $(P<0.05)$ are marked by an asterisk. 

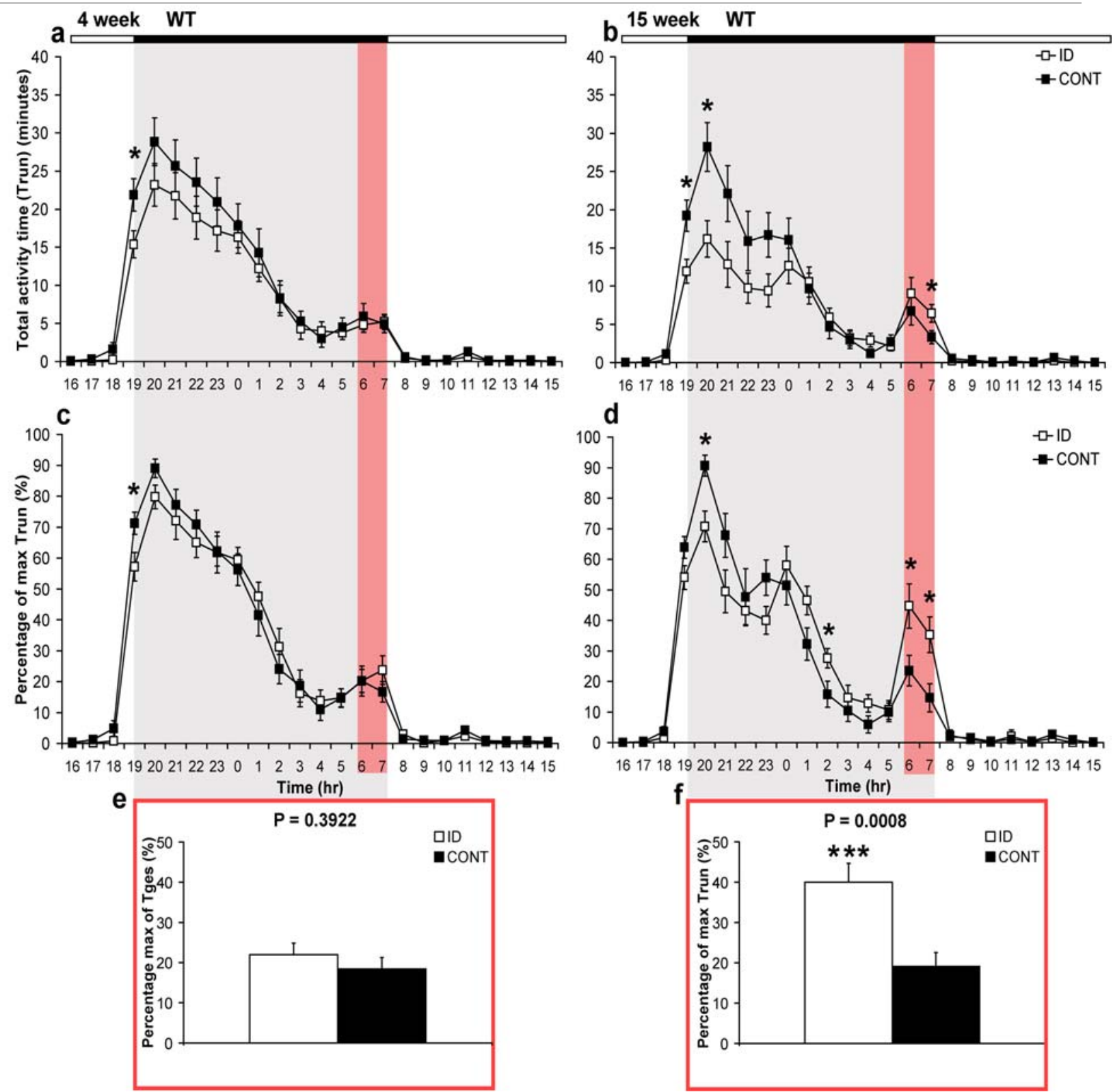

Fig. 2.7. ID diet alters the activity time of wheel-running during the late phase of the active cycle in WT mice. a,c,e. Wheel-running activity is presented for ID $(\square \pm \mathrm{SEM}, n=15)$ and control $(\square \pm \mathrm{SEM}, n=12)$ mice at a diet duration of 4 weeks P28, and b,d,f. for ID ( $\square \pm$ SEM, $n=14)$ and control $(\square \pm$ SEM, $n=11)$ mice at a diet duration of 15 weeks P28. The parameter shown, a,b. describes the total activity time $\left(\mathrm{T}_{\text {run }}\right)$ of mice on the running wheel at the two diet durations over a 24 hour time period (hr), as shown by the x-axis. From these absolute values (a,b.) relative values (c,d.) were calculated for every mouse independently c,d. showing the percentage of activity time, which is the time the mouse spent running in the hour when compared to the maximum recorded activity time it achieved during the period 19:00hr-03:00hr. Compared to the light phase (07:00hr-19:00hr), the nocturnal nature of mice show elevated running activity during the dark phase (19:00hr07:00hr), represented by the grey region projected from the black portion of the overhanging bar $(\mathbf{a}, \mathbf{b})$. The red highlighted portion of the grey region represents the final 2 hour period prior to the start of the light cycle (07:00) i.e. before mice rest. Values highlighted red, originating from c,d. are represented in the graphs within the corresponding red boxes, e,f. showing the difference $(P<0.05)$ ID diet has upon activity during the final 2 hour period prior to lights on. Significant differences $(P<0.05)$ are marked by an asterisk. 


\subsubsection{Wheel-running performance in $D 3^{-/-}$mice}

Similarly to WT mice, specific time periods will be focussed upon, especially those corresponding to the latter third $(03: 00 \mathrm{hr}-07: 00 \mathrm{hr})$ of the dark phase and when human RLS symptoms prevail. Furthermore, it may give insight into the importance of this receptor in RLS symptomatology and its interaction with iron-deficiency.

The voluntary wheel-running behaviour was assessed in both control and ID D3 $3^{-/-}$mice (Fig. 2.8) at two different durations of ID diet, 4 weeks (Fig. 2.8a,c,e) and 15 weeks (Fig. 2.8b,d,f). The parameters which were used to assess the moues's performance on the running wheel and whether it was influenced by an ID diet were, distance ran (Distance $(\mathrm{m})$ ), maximum running speed $\left(\mathrm{V}_{\max }(\mathrm{m} / \mathrm{s})\right)$ and finally the average distance per run (Distance $\left./ \mathrm{N}_{\text {run }}(\mathrm{m} / \mathrm{run})\right)$. At both 4 and 15 week durations, an influence of diet upon the performance was observed during the dark phase between ID and control mice (Fig. 2.8). Similarly to that observed in WT mice, the beginning period of the dark phase also showed a significant decrease in the Distance of ID $\mathrm{D}^{-/-}$mice, however this was found at both a 4 and 15 week duration of diet (Fig. 2.8a,b). Despite this change in Distance, $\mathrm{V}_{\max }$ remains very stable with the exception of ID mice showing a significant increase $(P<0.05)$ at both $03: 00 \mathrm{hr}$ and $04: 00 \mathrm{hr}$ (Fig. 2.8c,d). This increase during this period of the dark phase is closely correlated to the significant increase $(P$ $<0.05$ ) in Distance observed in ID D3 ${ }^{-/-}$mice at both 4 and 15 weeks diet durations (Fig. $2.8 \mathrm{a}, \mathrm{b})$.

In comparison to the stable Distance/ $\mathrm{N}_{\text {run }}$ observed between ID WT and control WT mice, ID D3 ${ }^{-/-}$compared to control D3 $3^{-/-}$mice show a significant decrease $(P<0.05)$ in this parameter, predominantly during the initial stage of the dark phase (Fig. 2.8e,f). Further differences in the wheel-running performance were observed between $\mathrm{WT}$ and $\mathrm{D}^{-/-}$mice. These differences were illustrated by means of an ANOVA. This showed no effect of time, diet and strain upon Distance $(P>0.05$; Table 2.3), although, such an interaction was shown for $\mathrm{V}_{\max }$, and Distance/ $\mathrm{N}_{\text {run }}(P<0.05$; Table 2.3). Therefore, a greater influence of ID diet upon the wheel-running performance was observed in $\mathrm{D}^{-/-}$compared to WT mice. In addition, the duration of diet had no further effect upon this difference as shown by the interaction of time, diet, strain and duration $(P>0.05$; Table 2.3$)$. 

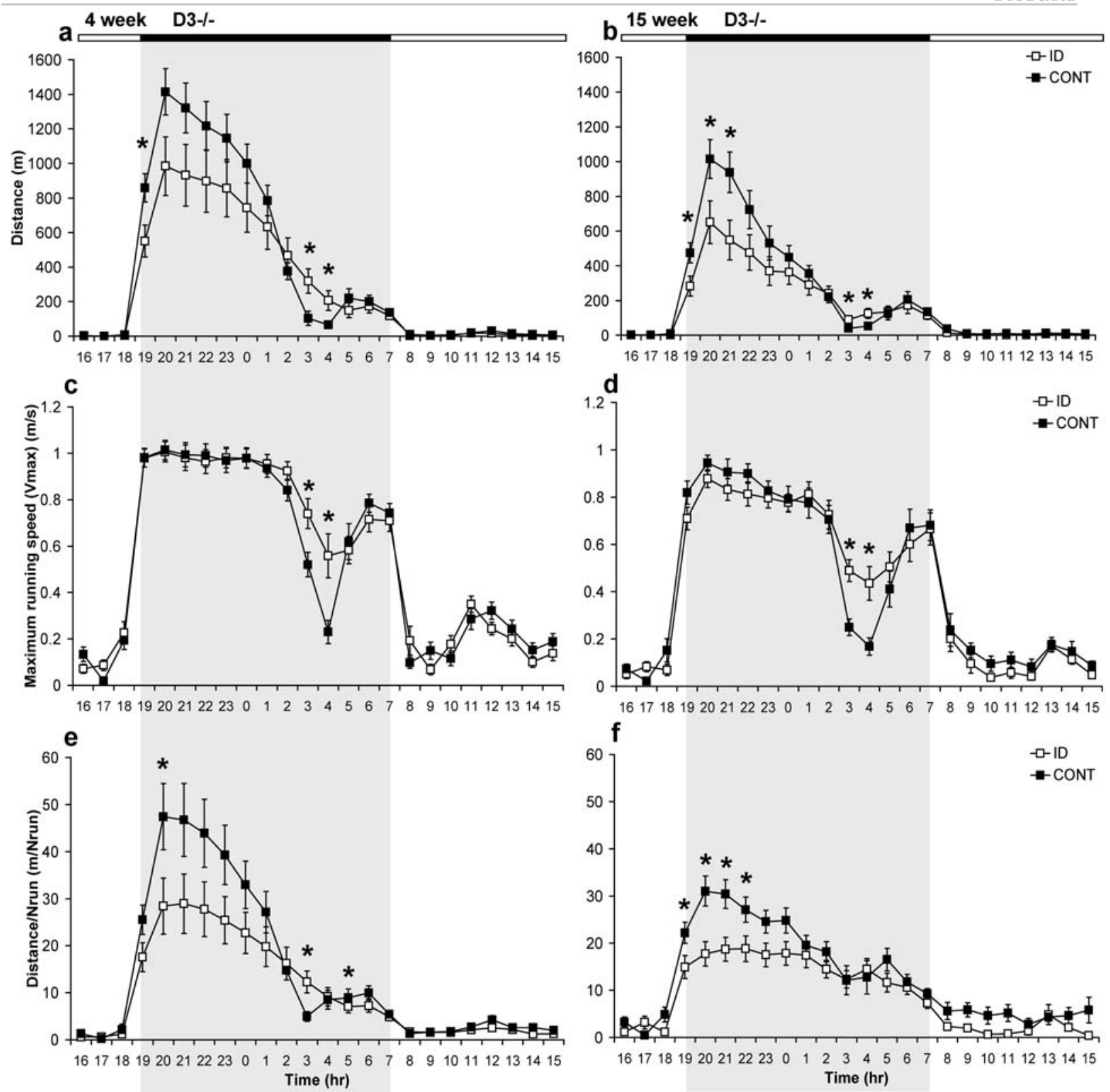

Fig. 2.8. Influence of ID diet and duration upon voluntary wheel-running performance in $\mathrm{D3}^{-/-}$mice. a,c,e. Wheel-running activity is presented for ID ( $\square \pm \mathrm{SEM}, n=15)$ and control $(\square \pm \mathrm{SEM}, n=13)$ mice at a diet duration of 4 weeks P28, and $\mathbf{b}, \mathbf{d}, \mathbf{f}$. for ID ( $\square \pm \mathrm{SEM}, n=14)$ and control $(\square \pm$ SEM, $n=13)$ mice at a diet duration of 15 weeks P28. Three different parameters were recorded at the two diet durations to assess the effect ID diet has upon the wheel-running performance over a 24 hour time period ( $\mathrm{hr}$ ), as shown by the x-axis, these were a,b. Total Distance ran (metres) for every hour, c,d. Maximum running speed (metres/second) and e,f. Average distance per run (metres/run). Compared to the light phase (07:00hr-19:00hr), the nocturnal nature of mice show elevated running performance during the dark phase $(19: 00 \mathrm{hr}-07: 00 \mathrm{hr})$, represented by the grey region projected from the black portion of the overhanging bar $(\mathbf{a}, \mathbf{b})$.

\subsubsection{Iron-deficiency increases wheel-running activity in $D 3^{-/-}$mice}

The activity behaviour of both control and ID D3 ${ }^{-/-}$mice (Fig. 2.9 and 2.11) was investigated at two different durations of ID diet, 4 weeks (Fig. 2.9a,c and 2.11a,c) and 15 weeks (Fig. 2.9b,d and $2.11 \mathrm{~b}, \mathrm{~d})$. The parameters used to show activity were; the number of runs $\left(\mathrm{N}_{\text {run }}\right)$ (Fig. 2.9a,b) and percentage of the maximum number of runs at each time-point (Percentage of max $\mathrm{N}_{\text {run }}(\%)$ ) (Fig. 2.9c,d). Moreover, the second set of parameters are the total activity time $\left(\mathrm{T}_{\text {run }}\right)$ spent running (Fig. 2.10a,b) and the percentage of the total active time at each time-point 
(Percentage of max $\mathrm{T}_{\text {run }}(\%)$ ) (Fig. 2.10c,d). The percentage parameters represent relative values derived from the absolute values $\left(\mathrm{N}_{\text {run }}\right.$ and $\left.\mathrm{T}_{\text {run }}\right)$. The purpose for creating these relative values for $\mathrm{D}^{-/-}$mice are for the same reasons as described for the WT mice (section 2.5.1.2.).

The running activity of ID D3 $3^{-/-}$mice at both 4 and 15 weeks diet shows a unique running profile at the 03:00hr-04:00hr time period, i.e. within the latter third of the dark phase. This period is highlighted within the red box as shown in Fig. 2.9a-d and 2.11a-d. The change in activity between ID and control D3 $3^{-/-}$mice was confirmed at both 4 and 15 week diet durations by an ANOVA, which illustrated an effect of the interaction between diet and time upon; $\mathrm{N}_{\text {run }}$, Percentage of $\max \mathrm{N}_{\text {run }}, \mathrm{T}_{\text {run }}$ and Percentage of $\max \mathrm{T}_{\text {run }}(P<0.05$; Table 2.4). Post $h o c$ tests revealed the activity of both ID and control groups during this 2 hour period, to be significantly reduced $(P<0.05)$ at 15 weeks diet duration when compared to 4 weeks diet duration. This was further verified by the interaction of time, strain and duration, whereby all parameters with the exception of $\mathrm{T}_{\text {run }}$ showed a significant decrease $(P<0.05$; Table 2.4). Also, the percentage of max $\mathrm{N}_{\text {run }}$ was significantly increased $(P<0.05)$ by 2 and 3 fold in ID $\mathrm{D}^{-/-}$compared to control D3 ${ }^{-/-}$mice for the 4 and 15 week diet durations, respectively (Fig. $2.9 \mathrm{e}, \mathrm{f})$. However, the significantly elevated $(P<0.05)$ Percentage of max $\mathrm{T}_{\text {run }}$ activity in ID $\mathrm{D}^{-/-}$compared to control $\mathrm{D}^{-/-}$mice remained similar between the 4 and 15 week diet durations during the period 03:00hr-04:00hr (Fig. 2.10e,f). This level of increased Percentage of max $\mathrm{T}_{\text {run }}$ activity of ID D3 ${ }^{-/-}$mice at both 4 (Fig. 2.10e) and 15 weeks (Fig. 2.10f) was approximately 4 fold greater than control $\mathrm{D} 3^{-/-}$mice. Interestingly, the Percentage of max $\mathrm{N}_{\text {run }}$ was shown to be elevated in ID D3 ${ }^{-/}$mice at 04:00hr after 4 weeks (Fig. 2.9c), however, after 15 weeks the significantly $(P<0.05)$ elevated activity in ID D3 ${ }^{-/-}$mice occurred 1 hour earlier, therefore at $03: 00 \mathrm{hr}$ (Fig. 2.9d). The Percentage of max $\mathrm{T}_{\text {run }}$ showed an elevated activity which started at 02:00hr and continued until 04:00hr. This was observed after both 4 and 15 weeks of diet.

In addition, ID D3 $3^{-/-}$mice also show a reduced $\mathrm{T}_{\text {run }}$ activity compared to control $\mathrm{D}^{-/-}$ mice during the initial part of the dark phase at both 4 (Fig. 2.10a) and 15 weeks (Fig. 2.10b) of diet. However, the relative value Percentage of $\max \mathrm{T}_{\text {run }}$, did not show ID D3 ${ }^{-/-}$mice to have a decreased activity during the initial stages of the dark phase (Fig. $2.10 \mathrm{c}, \mathrm{d}$ ). 

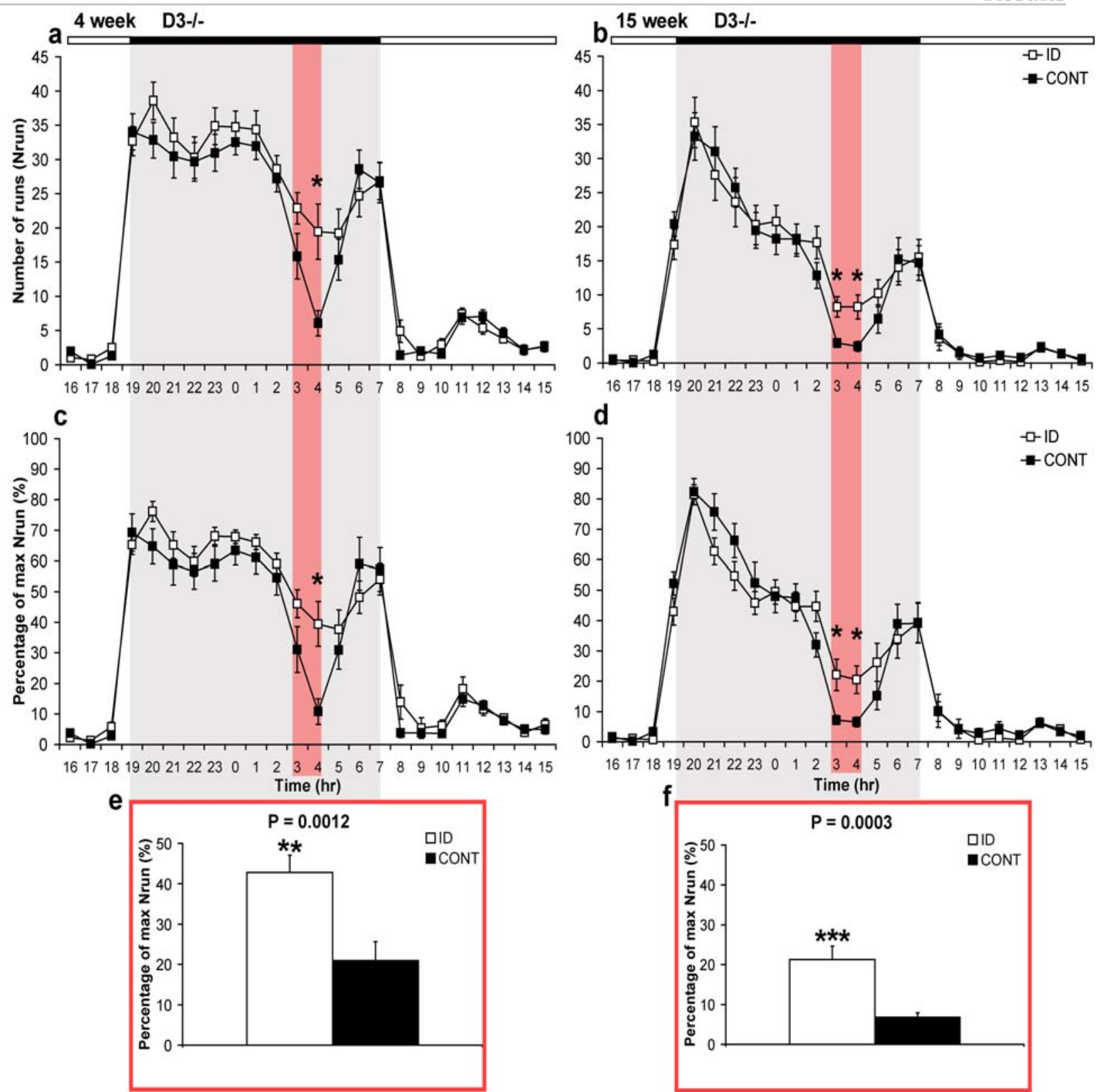

Fig. 2.9. ID diet alters the activity shown by number of runs during the dark phase in $\mathrm{D3}^{-/-}$mice. a,c,e. Wheel-running activity is presented for ID ( $\square \pm \mathrm{SEM}, n=15)$ and control $(\square \pm$ SEM, $n=13)$ mice at a diet duration of 4 weeks P28, and $\mathbf{b}, \mathbf{d , f}$. for ID ( $\square \pm \mathrm{SEM}, n=14)$ and control $(\square \pm$ SEM, $n=13)$ mice at a diet duration of 15 weeks P28. The parameter shown, a,b. describes the number of times the mice voluntarily ran $\left(\mathrm{N}_{\text {run }}\right)$ on the running wheel at the two diet durations over a 24 hour time period (hr), as shown by the x-axis. From these absolute values $(\mathbf{a}, \mathbf{b}$.) relative values $(\mathbf{c}, \mathbf{d}$.) were calculated for every mouse independently $\mathbf{c}, \mathbf{d}$. showing the percentage of runs, which is the number of runs the mouse ran in the hour when compared to the maximum recorded number of runs it achieved during the period 19:00hr-03:00hr. Compared to the light phase (07:00hr-19:00hr), the nocturnal nature of mice show elevated running activity during the dark phase (19:00hr-07:00hr), represented by the grey region projected from the black portion of the overhanging bar $(\mathbf{a}, \mathbf{b})$. The red highlighted portion of the grey region represents a 2 hour period during the active dark cycle. c,d. The values bound within the red highlighted region are represented in the graphs within the corresponding red boxes, e,f. showing the difference $(P<0.05)$ ID diet has upon activity during this specific 2 hour period during the dark cycle, which is 3 hours prior to the start of the light cycle $(07: 00 \mathrm{hr})$, when mice rest. Significant differences $(P<0.05)$ are marked by an asterisk. 

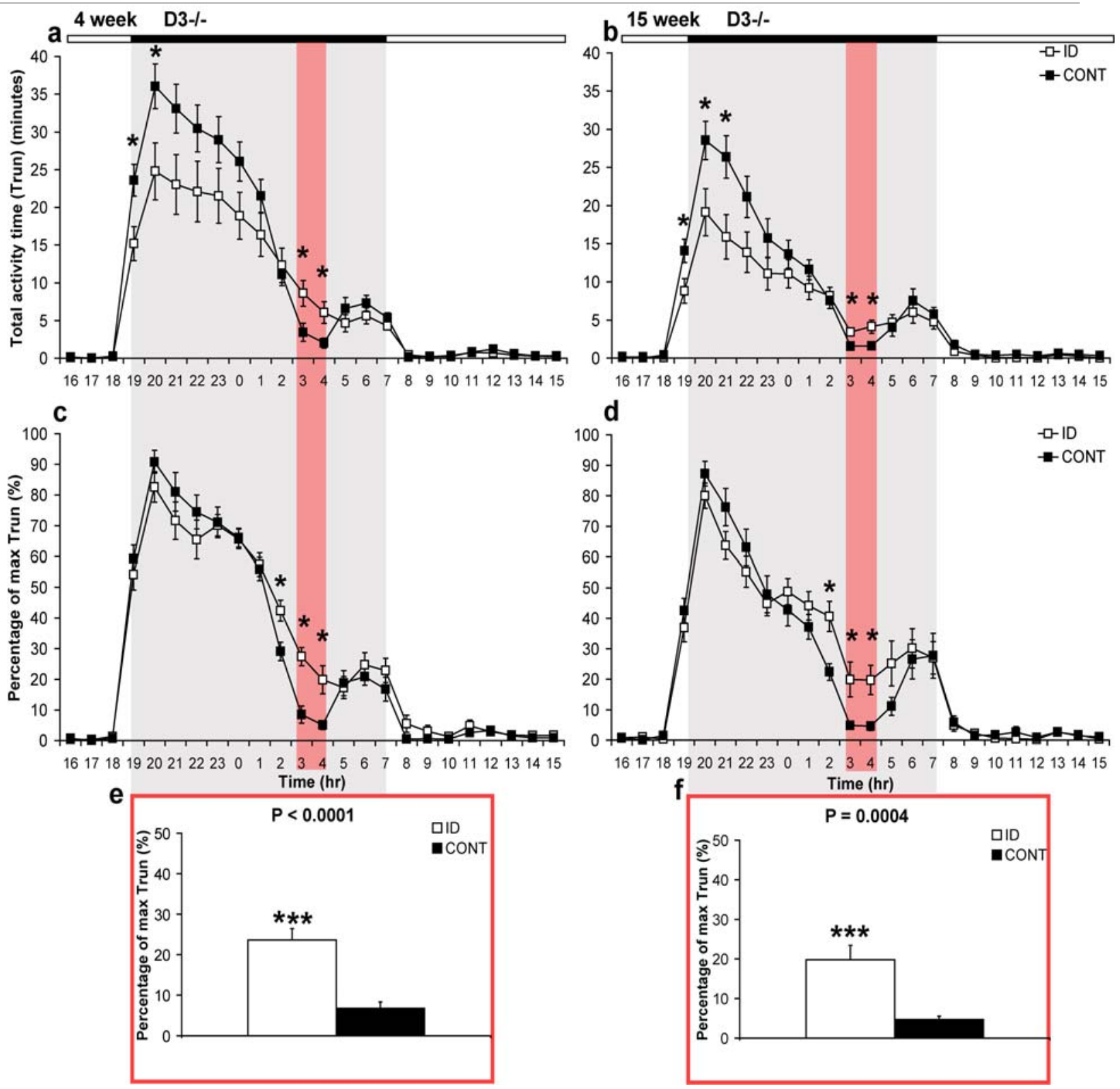

Fig. 2.10. ID diet alters the activity time of wheel-running during the dark phase in $\mathrm{D3}^{-/-}$mice. a,c,e. Wheelrunning activity is presented for ID ( $\square \pm \mathrm{SEM}, n=15)$ and control $(\square \pm \mathrm{SEM}, n=13)$ mice at a diet duration of 4 weeks P28, and b,d,f. for ID ( $\square \pm$ SEM, $n=14)$ and control $(\square \pm$ SEM, $n=13)$ mice at a diet duration of 15 weeks P28. The parameter shown, a,b. describes the total activity time $\left(\mathrm{T}_{\mathrm{run}}\right)$ of mice on the running wheel at the two diet durations over a 24 hour time period (hr), as shown by the x-axis. From these absolute values (a,b.) relative values (c,d.) were calculated for every mouse independently c,d. showing the percentage of activity time, which is the time the mouse spent running every hour when compared to the maximum recorded activity time it achieved during the period 19:00hr-03:00hr. Compared to the light phase $(07: 00 \mathrm{hr}-19: 00 \mathrm{hr})$, the nocturnal nature of mice show elevated running activity during the dark phase (19:00hr-07:00hr), represented by the grey region projected from the black portion of the overhanging bar $(\mathbf{a}, \mathbf{b})$. The red highlighted portion of the grey region represents a 2 hour period during the active dark cycle. c,d. The values bound within the red highlighted region are represented in the graphs within the corresponding red boxes, e,f. showing the difference $(P<0.05)$ ID diet has upon activity during this specific 2 hour period during the dark cycle, which is 3 hours prior to the start of the light cycle $(07: 00 \mathrm{hr})$, when mice rest. Significant differences $(P<0.05)$ are marked by an asterisk. 


\subsubsection{Differences in wheel-running activity between WT and D3 $3^{-/-}$mice}

The pattern (onset and offset) of running activity for WT and $\mathrm{D} 3^{-/-}$mice represented by a double plotted actogram (Fig. 2.11), appears relatively stable regardless of the diet duration or strain (WT or $\mathrm{D}^{-/-}$). No shift in activity onset and offset was observed prior to or after the dark phase, respectively. However, a noticeable increase in light phase activity for ID D3 $3^{-/-}$and control D3 ${ }^{-/-}$mice compared to ID WT and control WT mice was observed at 4 weeks ID diet duration P28. Also, this effect can be clearly seen in Fig. 2.9a,c for D3 $3^{-/-}$mice and Fig. 2.6a,c for WT mice.
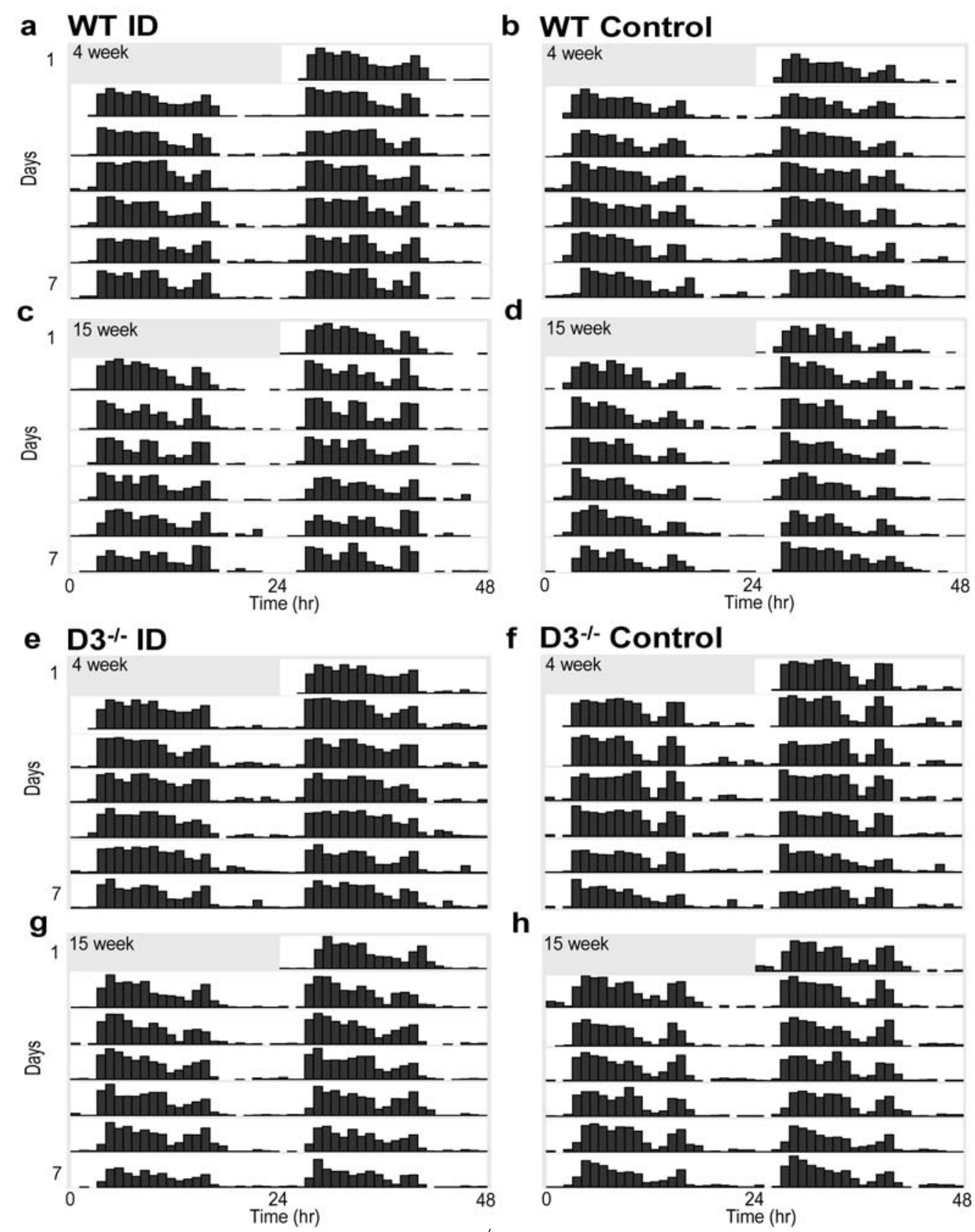

Fig. 2.11. Double plotted actograms of $\mathrm{WT}$ and $\mathrm{D}^{-/-}$mice. Heightened activity starts during the dark phase (19:00hr) as shown by large bars, where the start of the light phase $(07: 00 \mathrm{hr})$ characteristically shows a decreasing level of activity. The nocturnal activity cycle of mice is governed by a $12 \mathrm{hr} / 12 \mathrm{hr}$ light $/$ dark cycle. The differences in circadian running activity from 7 consecutive days corresponding to, a-d. WT and e-h. D ${ }^{-/-}$mice. Each day is plotted twice so that the activity of each day can be compared with the previous day; therefore activity is plotted over a $48 \mathrm{hr}$ period. The two different durations (4 weeks and 15 weeks) of diet P28 are shown. 
At a 4 week ID diet duration both WT and $\mathrm{D} 3^{-/-}$mice show strong running activity as shown by a greater level of activity during the dark phase (Fig. 2.11a,b,e,f). However, after a 15 week ID diet duration both strains appear to lose this heightened activity during the entire dark phase (Fig. 2.11c,d,g,h), where a change in the pattern of circadian wheel-running activity emerges, particularly at the latter third of the dark phase (03:00hr-07:00hr). This effect of a reduced activity over a longer diet duration (15 weeks) and the influence of diet, strain and duration is clearly shown in Fig. 2.12. Furthermore an ANOVA showed the interaction of time, strain and duration to have an effect upon $\mathrm{N}_{\text {run }}$, Percentage of $\max \mathrm{N}_{\text {run }}$ and Percentage of $\max \mathrm{T}_{\text {run }}(P<0.05$; Table 2.4). However, when diet is included in this interaction, no effect is observed for all activity parameters $(P>0.05$; Table 2.4$)$. Interestingly, in comparison to the other groups at the $06: 00 \mathrm{hr}-07: 00 \mathrm{hr}$ time period, ID WT mice appear to maintain a constant level of elevated activity (Percentage of $\max \mathrm{N}_{\text {run}}$ ) between 4 (Fig. 2.12e) and 15 week (Fig. 2.12f) ID diet durations. The ID WT activity is elevated above all other groups and in particular is observed at the 2 hour period prior to lights on $(07: 00 \mathrm{hr})$, which marks the start of the rest phase in nocturnal mice.

When comparing between groups, it appears that the ID D3 $3^{-/-}$mice have a unique voluntary wheel-running activity which is increased compared to control D3 ${ }^{-/-}$mice at the period 03:00hr-04:00hr (Fig. 2.9e,f). Post hoc tests revealed that ID D3 ${ }^{-/-}$mice show a significant $(P<0.05)$ increase in activity compared to ID WT mice at 4 weeks ID diet duration (Fig. 2.12e), where this effect is not present at 15 weeks of diet (Fig. 2.12f). This was further shown by the interaction effect of time, diet and strain which was significantly different for the parameters $\mathrm{N}_{\text {run }}$ and Percentage of $\max \mathrm{N}_{\text {run }}(P<0.05$; Table 2.4). Also, this effect was shown by compiling activity data into 2 hour time epochs and creating a factor called group, which includes strain and diet as a unified factor. The second time period (06:00hr-07:00hr) describes a significant increase $(P<0.05)$ in activity (Percentage of max $\mathrm{N}_{\text {run }}$ ) of control D3 $3^{-/-}$ compared to control WT mice (Fig. 2.12e,f). Therefore, the level of activity changes according to strain and ID or control diets.

The second set of activity parameters $T_{\text {run }}$ and Percentage of $\max T_{\text {run }}$ (Fig. 2.13) show a unique activity profile at 03:00hr-04:00hr, which is increased in ID D3 ${ }^{-/-}$mice compared to control D3 $3^{-/-}$mice. Post hoc tests show at this time-point that both ID D3 $3^{-/-}$and control D3 $3^{-/-}$ mice have a significantly $(P<0.05)$ greater activity compared to ID WT and control WT groups. This difference was only present at 4 weeks diet (Fig. 2.13e). This was further shown by the interaction effect of time, diet and strain which proved significantly different for Percentage of max $\mathrm{T}_{\text {run }}\left(P<0.05\right.$; Table 2.4), however not for $\mathrm{T}_{\text {run }}(P>0.05$; Table 2.4). Observing the ID WT mouse group during the 06:00hr-07:00hr time-point shows, that after 15 weeks of diet (Fig. 2.13f) Percentage of max $\mathrm{T}_{\text {run }}$ is significantly elevated $(P<0.05)$ by 
approximately 2 fold when compared to the value at 4 weeks (Fig. 2.13e). This increase in activity time is observed when Percentage of max $\mathrm{N}_{\text {run }}$ remains stable between the different diet durations (Fig. 2.12e,f).
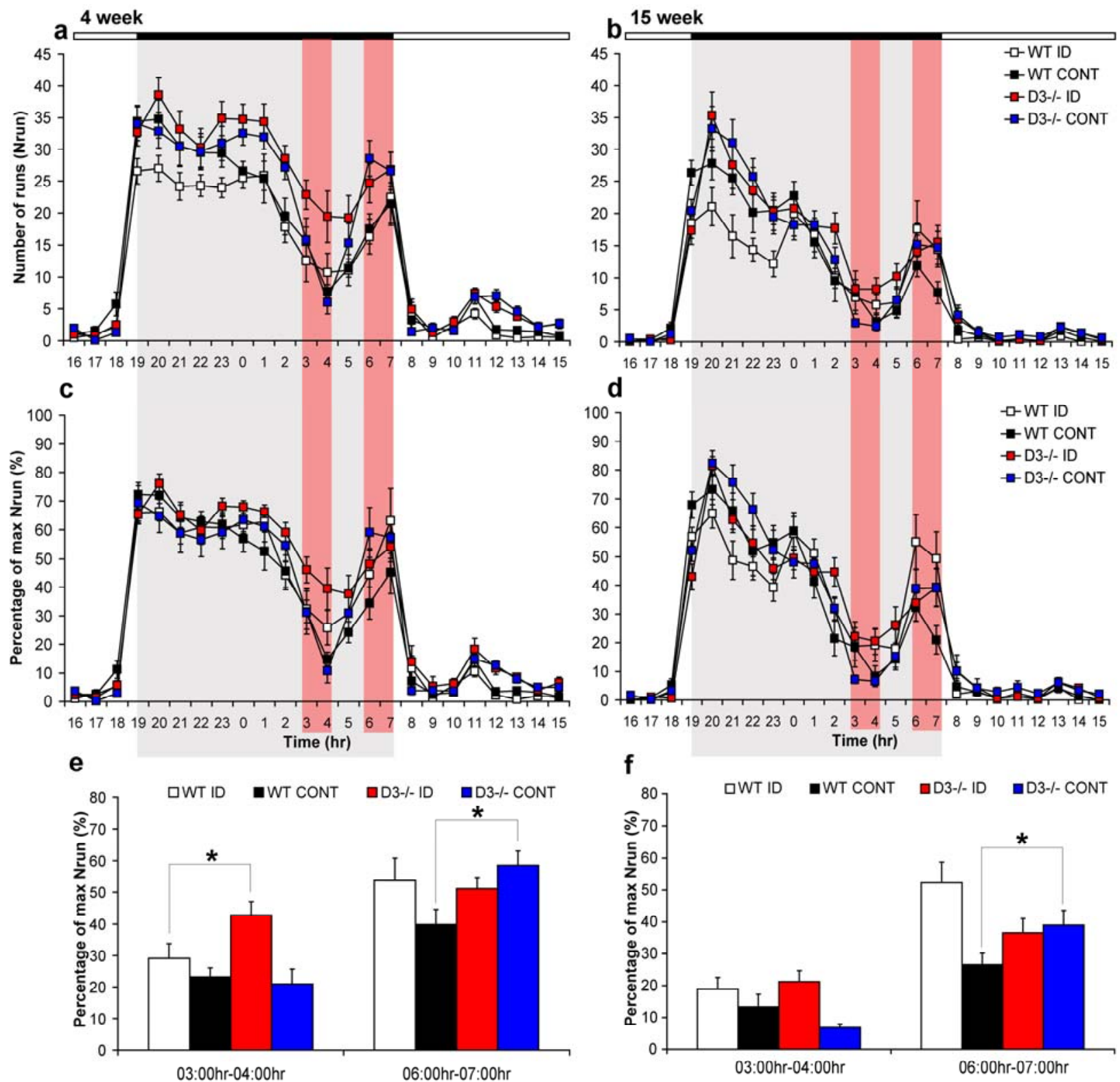

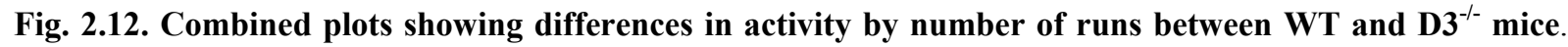

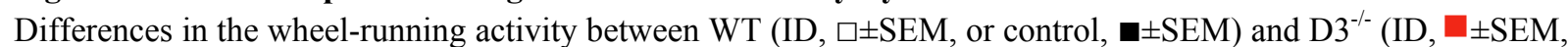

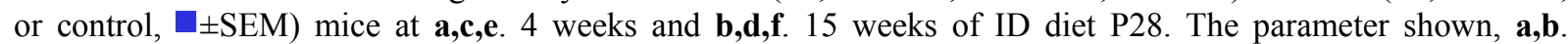
describes the number of times mice voluntarily ran $\left(\mathrm{N}_{\mathrm{run}}\right)$ on the running wheel at the two diet durations over a 24 hour time period (hr), as shown by the x-axis. From these absolute values (a,b.) relative values (c,d.) were calculated for every mouse independently c,d. showing the percentage of runs, which is the number of runs the mouse ran in the hour when compared to the maximum recorded number of runs it achieved during the period 19:00hr-03:00hr. Compared to the light phase (07:00hr-19:00hr), the nocturnal nature of mice show elevated running activity during the dark phase $(19: 00 \mathrm{hr}-07: 00 \mathrm{hr})$, represented by the grey region projected from the black portion of the overhanging bar (a,b). The two red highlighted portions of the grey region each represents a 2 hour period during the dark cycle. c,d. The values bound within the red highlighted region are represented in the corresponding graphs below e,f. showing the running activity differences (\%) between the strain of mice (WT or $\left.\mathrm{D}^{-/-}\right)$at two time-points $(03: 00 \mathrm{hr}-04: 00 \mathrm{hr}$ and $06: 00 \mathrm{hr}-07: 00 \mathrm{hr})$ during the dark phase prior to the start of the light cycle $(07: 00 \mathrm{hr})$, which is when mice rest. Significant differences $(P<0.05)$ are marked by an asterisk. 

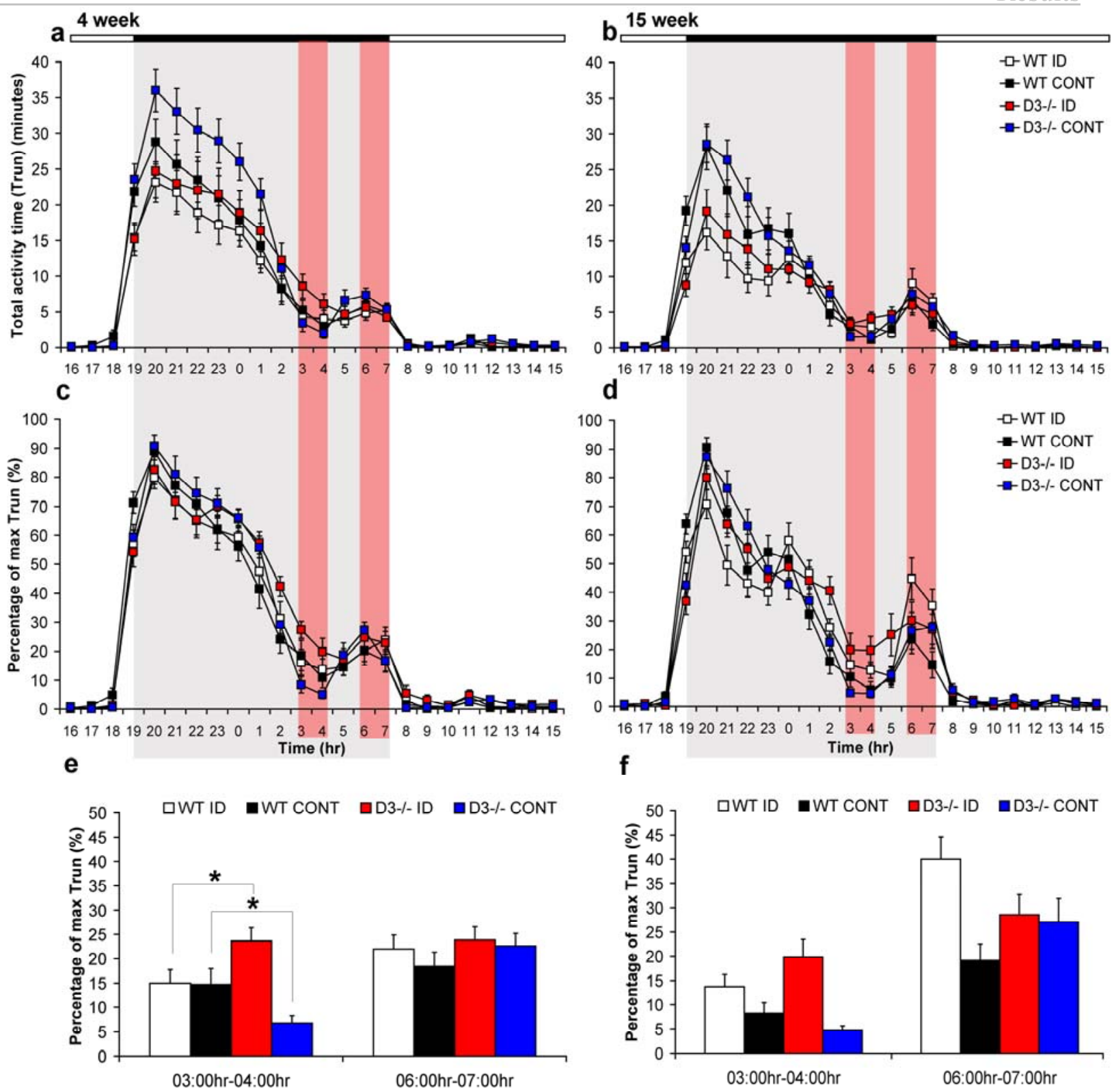

Fig. 2.13. Combined plots showing differences in wheel-running activity time between $\mathrm{WT}$ and $\mathrm{D3}^{-/-}$mice. Differences in the wheel-running activity between WT (ID, $\square \pm$ SEM, or control, $\square \pm$ SEM) and D3 ${ }^{-/-}$(ID, $\square \pm$ SEM,

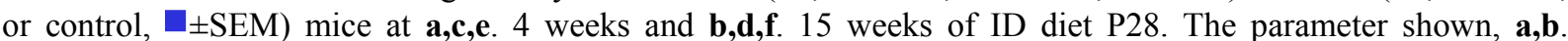
describes the total activity time $\left(\mathrm{T}_{\text {run }}\right)$ of mice on the running wheel at the two diet durations over a 24 hour time period (hr), as shown by the x-axis. From these absolute values (a,b.) relative values (c,d.) were calculated for every mouse independently c,d. showing the percentage activity time, which is the time the mouse spent running in the hour when compared to the maximum activity time it achieved during the period 19:00hr-03:00hr. Compared to the light phase (07:00hr-19:00hr), the nocturnal nature of mice show elevated running activity during the dark phase (19:00hr-07:00hr), represented by the grey region projected from the black portion of the overhanging bar $(\mathbf{a}, \mathbf{b})$. The two red highlighted portions of the grey region each represents a 2 hour period during the dark cycle. c,d. The values bound within the red highlighted region are represented in the corresponding graphs below e,f. showing the running activity differences $(\%)$ between the strain of mice (WT or D3 ${ }^{-/}$) at two time-points $(03: 00 \mathrm{hr}-04: 00 \mathrm{hr}$ and $06: 00 \mathrm{hr}-07: 00 \mathrm{hr})$ during the dark phase prior to the start of the light cycle $(07: 00 \mathrm{hr})$, which is when mice rest. Significant differences $(P<0.05)$ are marked by an asterisk. 
Table 2.3

Results of the ANOVAs-Voluntary wheel-running performance

Voluntary wheel-running - Performance Parameters

Distance

Time

Diet

Strain

Duration

Time $x$ Diet

Time $x$ Strain

Time $x$ Duration

Diet $x$ Strain

Diet $x$ Duration

Strain $x$ Duration

Time $x$ Diet $x$ Strain

Time $x$ Diet $x$ Duration

Time $x$ Strain $x$ Duration

Diet $x$ Strain $x$ Duration

Time $\times$ Diet $x$ Strain $\times$ Duration

$\underline{\max }$

Time

Diet

Strain

Duration

Time $\times$ Diet

Time $\times$ Strain

Time $\times$ Duration

Diet $x$ Strain

Diet $x$ Duration

Strain $\times$ Duration

Time $\times$ Diet $\times$ Strain

Time $\times$ Diet $x$ Duration

Time $\times$ Strain $\times$ Duration

Diet $x$ Strain $x$ Duration

Time $\times$ Diet $x$ Strain $x$ Duration

\section{Distance/Nrun}

Time

Diet

Strain

Duration

Time $\times$ Diet

Time $\times$ Strain

Time $\times$ Duration

Diet $x$ Strain

Diet $x$ Duration

Strain $\times$ Duration

Time $\times$ Diet $x$ Strain

Time $\times$ Diet $x$ Duration

Time $\times$ Strain $\times$ Duration

Diet $x$ Strain $x$ Duration

Time $\times$ Diet $x$ Strain $x$ Duration
Degrees of freedom

11

1

1

1

11

11

11

1

1

1

11

11

11

1

11

Degrees of freedom

11

1

1

1

11

11

11

1

1

1

11

11

11

1

11

Degrees of freedom

11

1

1

1

11

11

11

1

1

1

11

11

11

1

11

$\begin{array}{cr}F \text { values } & P \text { values } \\ 191.446 & <0.001^{*} \\ 4.313 & 0.040^{*} \\ 6.038 & 0.016^{*} \\ 18.606 & <0.001^{*} \\ 11.027 & <0.001^{*} \\ 3.520 & <0.001^{*} \\ 12.826 & <0.001^{*} \\ .178 & 0.674 \\ .042 & 0.838 \\ 1.315 & 0.254 \\ .967 & 0.475 \\ .544 & 0.874 \\ 1.461 & 0.140 \\ .026 & 0.871 \\ .785 & 0.656\end{array}$

$F$ values

$P$ values

$152.204<0.001$ *

$.036 \quad 0.851$

$.435 \quad 0.511$

$30.959<0.001$ *

$4.592<0.001$ *

$4.934<0.001$ *

2.0020 .025 *

$1.320 \quad 0.253$

$.661 \quad 0.418$

$.041 \quad 0.840$

2.6600 .002 *

$.856 \quad 0.584$

$1.045 \quad 0.404$

$1.220 \quad 0.272$

.897

0.543

${ }^{*} P<0.05$.

$F$ values $\quad P$ values

$111.966<0.001$ *

$4.042 \quad 0.047$ *

$1.020 \quad 0.315$

$1.401 \quad 0.239$

$5.809<0.001$ *

$1.626 \quad 0.086$

$12.977<0.001$ *

$1.489 \quad 0.225$

$.084 \quad 0.773$

$.632 \quad 0.429$

$3.216<0.001$ *

$.674 \quad 0.764$

$2.095 \quad 0.018^{*}$

$.097 \quad 0.756$

$1.158 \quad 0.312$ 
Table 2.4

Results of the ANOVAs-Voluntary wheel-running activity Voluntary wheel-running - Activity Parameters

\begin{tabular}{|c|c|c|c|c|c|c|c|c|c|}
\hline \multicolumn{2}{|c|}{ Nrun } & \multirow{2}{*}{$\begin{array}{c}\text { Degrees of freedom } \\
11\end{array}$} & \multicolumn{2}{|c|}{$F$ values $\quad P$ values } & \multicolumn{2}{|r|}{$\% \max$ Nrun } & \multirow{2}{*}{ Degrees of freedom } & $F$ values & $P$ values \\
\hline & $\overline{\text { Time }}$ & & 84.978 & $<0.001^{*}$ & & $\overline{\text { Time }}$ & & 91.483 & $<0.001^{*}$ \\
\hline & Diet & 1 & .052 & 0.820 & & Diet & 1 & 3.350 & 0.070 \\
\hline & Strain & 1 & 17.329 & $<0.001^{*}$ & & Strain & 1 & 3.909 & 0.051 \\
\hline & Duration & 1 & 55.898 & $<0.001^{*}$ & & Duration & 1 & 23.876 & $<0.001^{*}$ \\
\hline & Time $\times$ Diet & 11 & 3.330 & $<0.001^{*}$ & & Time $\times$ Diet & 11 & 3.732 & $<0.001^{*}$ \\
\hline & Time $x$ Diet(4) & 11 & 1.076 & 0.381 & & Time $\times$ Diet(4) & 11 & 1.027 & 0.423 \\
\hline & Time $x$ Diet(15) & 11 & 2.893 & $0.001^{*}$ & & Time $x \operatorname{Diet}(15)$ & 11 & 3.122 & $0.001^{*}$ \\
\hline & Time $x$ Strain & 11 & 1.351 & 0.191 & & Time $\times$ Strain & 11 & .951 & 0.490 \\
\hline & Time $x$ Duration & 11 & 3.247 & $<0.001^{\star}$ & & Time $\mathrm{x}$ Duration & 11 & 4.989 & $<0.001$ * \\
\hline & Diet $x$ Strain & 1 & 3.191 & 0.077 & & Diet x Strain & 1 & .008 & 0.930 \\
\hline & Diet $x$ Duration & 1 & .010 & 0.921 & & Diet x Duration & 1 & .548 & 0.461 \\
\hline & Strain $\times$ Duration & 1 & 1.444 & 0.232 & & Strain $\times$ Duration & 1 & .198 & 0.657 \\
\hline & Time $\times$ Diet $\times$ Strain & 11 & 2.705 & $0.002^{*}$ & & Time $\times$ Diet $\times$ Strain & 11 & 3.661 & $<0.001^{*}$ \\
\hline & Time $\times$ Diet $\times$ Duration & 11 & 1.014 & 0.431 & & Time $\times$ Diet $\times$ Duration & 11 & 1.562 & 0.105 \\
\hline & Time $\times$ Strain $\times$ Duration & 11 & 2.840 & $0.001^{*}$ & & Time $\times$ Strain $\times$ Duration & 11 & 2.565 & $0.003^{*}$ \\
\hline & Diet $x$ Strain $\times$ Duration & 1 & .290 & 0.592 & & Diet $x$ Strain $\times$ Duration & 1 & .362 & 0.549 \\
\hline & Time $\times$ Diet $\times$ Strain $\times$ Duration & 11 & .188 & 0.998 & & Time $\times$ Diet $\times$ Strain $\times$ Duration & 11 & .197 & 0.998 \\
\hline \multirow[t]{2}{*}{$2 \mathrm{hr}$} & Time & 6 & 62.640 & $<0.001^{\star}$ & $2 \mathrm{hr}$ & Time & 6 & 70.365 & $<0.001^{\star}$ \\
\hline & Time $\times$ Group & 18 & 3.065 & $<0.001^{*}$ & & Time $\times$ Group & 18 & 2.886 & $<0.001^{*}$ \\
\hline & Trun & Degrees of freedom & $F$ values & $P$ values & & $\% \max$ Trun & Degrees of freedom & $F$ values & $P$ values \\
\hline & $\overline{\text { Time }}$ & 11 & 208.159 & $<0.001^{*}$ & & $\overline{\text { Time }}$ & 11 & 229.762 & $<0.001^{*}$ \\
\hline & Diet & 1 & 5.811 & $0.018^{*}$ & & Diet & 1 & 1.983 & 0.162 \\
\hline & Strain & 1 & 3.676 & 0.058 & & Strain & 1 & 6.075 & $0.015^{*}$ \\
\hline & Duration & 1 & 12.946 & $0.001^{*}$ & & Duration & 1 & 16.336 & $<0.001$ * \\
\hline & Time $\times$ Diet & 11 & 13.179 & $<0.001^{*}$ & & Time $\times$ Diet & 11 & 7.004 & $<0.001^{*}$ \\
\hline & Time $x$ Diet(4) & 11 & .893 & 0.548 & & Time $x$ Diet(4) & 11 & .784 & 0.656 \\
\hline & Time $x$ Diet(15) & 11 & 4.748 & $<0.001 *$ & & Time $x \operatorname{Diet}(15)$ & 11 & 3.991 & $<0.001$ * \\
\hline & Time $\times$ Strain & 11 & 1.960 & $0.029 *$ & & Time $\times$ Strain & 11 & 1.077 & 0.376 \\
\hline & Time $x$ Duration & 11 & 8.644 & $<0.001 *$ & & Time $\times$ Duration & 11 & 6.856 & $<0.001$ * \\
\hline & Diet $x$ Strain & 1 & .267 & 0.607 & & Diet $x$ Strain & 1 & .248 & 0.619 \\
\hline & Diet x Duration & 1 & .018 & 0.894 & & Diet x Duration & 1 & .514 & 0.475 \\
\hline & Strain $\times$ Duration & 1 & 1.012 & 0.317 & & Strain $\times$ Duration & 1 & .208 & 0.650 \\
\hline & Time $\times$ Diet $\times$ Strain & 11 & 1.166 & 0.306 & & Time $\times$ Diet $\times$ Strain & 11 & 1.725 & 0.063 \\
\hline & Time $x$ Diet $\times$ Duration & 11 & .746 & 0.695 & & Time $x$ Diet $x$ Duration & 11 & 1.077 & 0.376 \\
\hline & Time $\times$ Strain $\times$ Duration & 11 & 1.533 & 0.114 & & Time $\times$ Strain $\times$ Duration & 11 & 2.007 & 0.025 * \\
\hline & Diet $\times$ Strain $\times$ Duration & 1 & .125 & 0.725 & & Diet $\times$ Strain $\times$ Duration & 1 & .000 & 0.996 \\
\hline & Time $x$ Diet $\times$ Strain $\times$ Duration & 11 & .897 & 0.543 & & Time $\times$ Diet $\times$ Strain $\times$ Duration & 11 & .749 & 0.691 \\
\hline $2 \mathrm{hr}$ & Time & 6 & 85.919 & $<0.001^{\star}$ & $2 \mathrm{hr}$ & Time & 6 & 108.455 & $<0.001^{\star}$ \\
\hline & Time $\times$ Group & 18 & 4.039 & $<0.001^{*}$ & & Time $\times$ Group & 18 & 3.383 & $<0.001 *$ \\
\hline
\end{tabular}

${ }^{*} p<0.05$.

(4) -4 Week WT Group

(15) - 15 Week WT Group

$2 \mathrm{hr}$ - Data in 2 hour time epochs after 15 weeks ID diet

Group - Diet x Strain 


\subsubsection{Increased ChAT immunoreactivity upon motoneurons at spinal T13-L2}

The motoneurons of the ventral horn at the spinal T13-L2 region are known to be in close apposition to and innervated by interneurons which form the CPG network. This region as previously mentioned (Introduction 1.3.3) is important in fine-tuning and execution of locomotion. Mice that performed in voluntary wheel-running experiments for 4 or 15 week periods of diet were used for the following experiments in determining whether irondeficiency induces a change in motoneuron function. In addition, the autonomic motoneurons of the IML from lamina VII, were quantified. These IML motoneurons form part of the sympathetic preganglionic pathways, where diencephalic A11-A14 neuron groups, the former known to be involved in RLS, project to this spinal region (Kalen et al., 1988).

At the T13-L2 spinal region one can clearly observe motoneurons (Fig. 2.14e), moreover, at the ventral horn ChAT immunoreactive (ChAT-IR) boutons were shown to contact motoneurons (Fig. 2.14a-d). This study was concerned with identifying changes in motoneuron area and ChAT-IR bouton innervation upon these ventral motoneurons, for both control and ID groups at 4 and 15 weeks of diet. Concerning area 1 (IML) (see Fig. 2.14e), the ANOVA showed no effects of duration, diet as well as the interaction of duration and diet upon the motoneuron area $(P>0.05$; Table 2.5), as shown in Fig. 2.14f. The ANOVA similarly showed the motoneuron area of the second region, area 2 (see Fig. 2.14e) to be uninfluenced by duration or diet as well as the interaction of duration and diet $(P>0.05$; Table 2.5), as shown in Fig. 2.14g. However, at this same ventral region of the spinal cord, the innervation density of ChAT-IR boutons upon motoneurons (Fig. 2.14h) was shown to be influenced by duration or diet $(P<0.05$; Table 2.5$)$, however no interaction effect between duration and diet was observed $(P>0.05$; Table 2.5). A post hoc test revealed a significant increase $(P<0.05)$ in ChAT-IR bouton density for ID compared to control WT mice at the 15 weeks diet duration. 

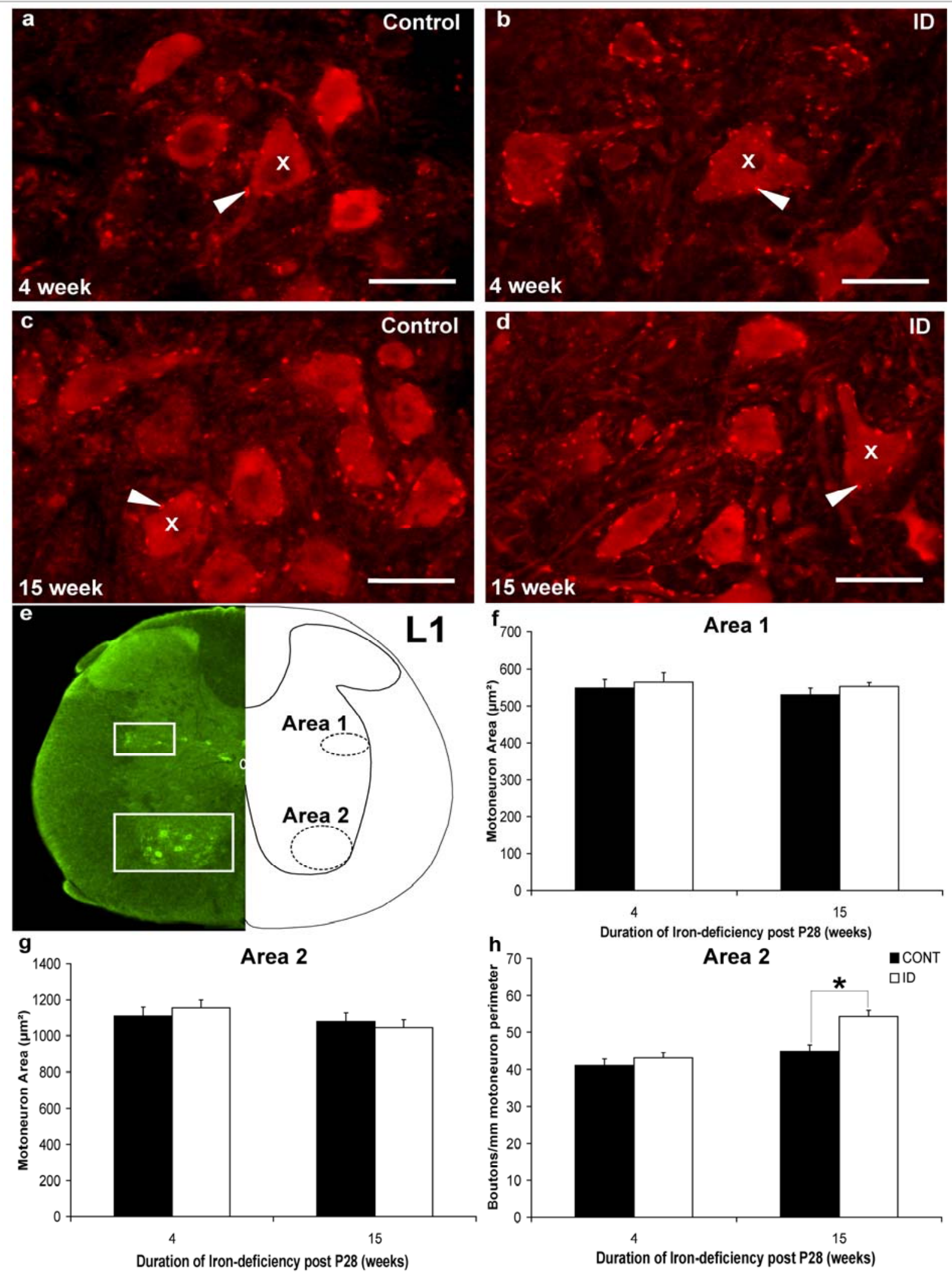

Fig. 2.14. The effect of ID diet on ChAT-IR expression upon motoneurons at the CPG region in WT mice. After wheel-running experiments, the T13-L2 spinal region from these mice were assessed for changes in motoneuron area and innervation at two regions, e. These regions are shown under fluorescence using fluorescein isothiocyanate (FITC). Firstly the motoneuron area at, f. area 1 (IML), shows differences between control ( \pm SEM, $n=7$ ) and ID mice ( $\square \pm$ SEM, $n=7$ ) at both 4 and 15 weeks of diet. The second region denoted as area 2, shows large ventral motoneurons. g. The motoneuron area at this region was also quantified between control $(\square \pm \mathrm{SEM}, n=7)$ and ID mice ( $\square \pm \mathrm{SEM}, n=7)$ at both 4 and 15 weeks of diet. a-d. Upon motoneurons (marked by $\mathrm{X}$ ) at area 2, ChAT-IR boutons (white arrow heads) can be clearly seen. $\mathbf{h}$. Also at area 2, the number of ChATIR boutons $/ \mathrm{mm}$ of motoneuron perimeter were quantified for both control $(\square \pm$ SEM, $n=7)$ and ID mice ( $\square \pm$ SEM,

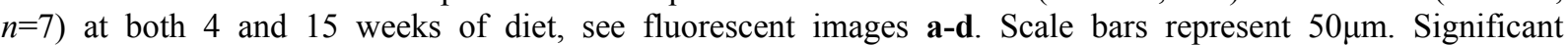
differences $(P<0.05)$ are marked by an asterisk. 
Table 2.5

Results of the ANOVAs

\section{ChAT-IR}

Motoneuron Area 1 (IML):

Duration

Diet

Duration x Diet

Motoneuron Area 2:

Duration

Diet

Duration x Diet

$\begin{array}{ccc}\text { Degrees of freedom } & F \text { values } & P \text { values } \\ 1 & 0.862 & 0.354 \\ 1 & 1.387 & 0.240 \\ 1 & 0.029 & 0.865\end{array}$

Motoneuron Area 2: Innervation density

Duration

Diet

Duration x Diet

Degrees of freedom

$\begin{array}{cc}F \text { values } & P \text { values } \\ 2.405 & 0.122 \\ 0.019 & 0.891 \\ 0.821 & 0.366\end{array}$

${ }^{*} P<0.05$.

$\begin{array}{ccc}\text { Degrees of freedom } & F \text { values } & P \text { values } \\ 1 & 10.989 & 0.001^{*} \\ 1 & 6.456 & 0.012^{*} \\ 1 & 2.624 & 0.106\end{array}$




\subsection{Assessing protein expression differences in the CNS}

\subsubsection{2-D DIGE Gel electrophoresis of mouse midbrain}

\subsubsection{Altered protein expression in ID WT mice at 15 weeks diet duration}

After completing in-silico analysis of the 2-D gels using the Decyder ${ }^{\mathrm{TM}} 5.02$ program (see section 4.8.4), a list of potential targets (spots) showing protein expression (spot intensity) differences between control and ID WT groups were displayed. In total 14 spots were selected based on the pre-set filters as described in the methods (section 4.8.4). A representative image of a gel which was used for analysis and protein spot selection is shown below (Fig. 2.15).

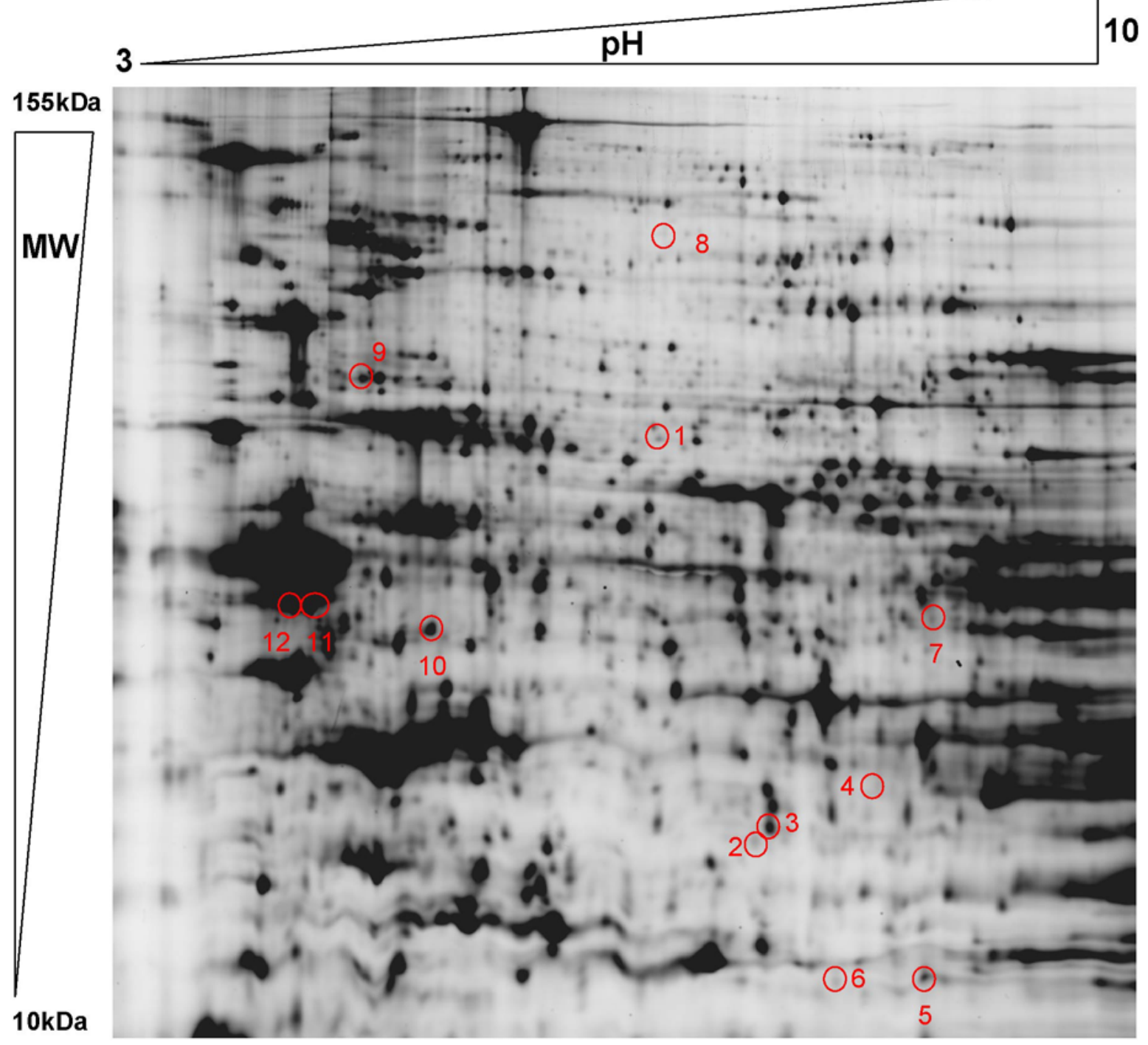

Fig. 2.15. 2-D DIGE gel showing protein expression differences of the midbrain from control WT and ID WT mice at 15 weeks diet duration. Protein content within whole midbrain lysate from a control WT and ID WT mouse was separated initially by isoelectric focussing (IEF) using first dimension (1-D) electrophoresis at a pH range from 3-10 (shown by top gradient bar) followed by a 2-D gel electrophoresis procedure, where proteins were separated at the second dimension (2-D) according to their varying molecular weight (155 kilodaltons (kDa)-10kDa, left hand side gradient bar). This figure represents a scanned 2-D gel, where all spots showing differences in protein expression between ID and control WT groups are indicated by a red circle. The red circles indicate the selected spots for picking and subsequent protein identification. 
The ID/C ratio and P-values relating to the 12 spots (shown in Fig. 2.15.) are shown below in Table 2.6. The additional 2 spots described below (Table 2.6A) represent the first findings from the initial 2-D DIGE experiment. The average ratio between ID and control (C) mice, hence (ID/C), indicates the level of expression for this protein between the groups. Therefore, spots $1,2,3,5,9,11,12$ (Table 2.6B) and 2 (Table 2.6A) show ratio values above 1.00, meaning that ID mice show a greater protein expression (upregulation) compared to controls at that particular spot. The remaining spots 4, 6, 7, 8, 10 (Table 2.6B) and 1 (Table 2.6A) show ID/C values below 1.00 meaning that the opposite effect occurs, therefore, ID mice display a reduced expression (downregulation) of this unidentified protein compared to control mice. Corresponding P-values identify whether the expression change of the unidentified protein at that spot is significant $(P<0.05)$ or not $(P>0.05)$. Moreover, not every spot showed a high level of significance as shown by the P-values below, however both spots 1 (Table 2.6A) and 10 (Table 2.6B) indicate a significant decrease $(P<0.05)$ in the expression of these two proteins in the midbrain from ID WT mice.

\begin{tabular}{|c|c|c|}
\hline Spot Number & Average Ratio (ID/C) & P-Value \\
\hline A) & 0.80 & $0.009^{*}$ \\
\hline 2 & 1.30 & 0.095 \\
\hline В) & 1.40 & 0.092 \\
\hline 2 & 1.20 & 0.097 \\
\hline 3 & 1.30 & 0.150 \\
\hline 4 & 0.72 & 0.063 \\
\hline 5 & 1.35 & 0.590 \\
\hline 6 & 0.69 & 0.079 \\
\hline 7 & 0.80 & 0.100 \\
\hline 8 & 0.60 & 0.120 \\
\hline 9 & 1.33 & 0.086 \\
\hline 10 & 0.74 & $0.021 *$ \\
\hline 11 & 1.33 & 0.150 \\
\hline 12 & 1.34 & 0.220 \\
\hline
\end{tabular}

Table 2.6. Differences in protein expression between control WT and ID WT mice. After completion of 2-D DIGE the selected spots showing protein expression differences were subjected to post-2-D gel electrophoresis in-silico analysis. This table illustrates the difference in protein expression as a ratio (ID/C) between ID and control (C) WT mice. The lower the ratio the greater the expression in control compared to ID animals and when the ID/C ratio is higher the opposite effect is present. A) The first 2-D DIGE experiment shows two identified spots, where the B) second experiment shows 12 spots as illustrated in Fig. 2.15. Significant differences $(P<$ 0.05 ) are marked by an asterisk.

After completing the in-silico analysis and identifying changes in protein expression between control and ID WT groups, protein samples were then identified using MALDI-TOF-MS. 


\subsubsection{Identification of 2-D DIGE protein samples from WT mouse midbrain by using MALDI-TOF-MS}

\subsubsection{MASCOT score analysis}

The minimal requirement for accepting a protein as identified was at least one peptide sequence match above identity threshold in coincidence with at least $20 \%$ sequence coverage in the peptide mass fingerprint (Jahn et al., 2006).

After completing the 2-D DIGE experiments, MALDI-TOF-MS and subsequent insilico analysis identified the proteins within the selected spots from 2-D DIGE (see methods section 4.9). Each protein's MASCOT score, sequence coverage, mass and isoelectric point (pI) were evaluated, as illustrated in Table 2.7. The sequence coverage refers to the identified peptides of the protein and the percentage of these identified peptides in relation to the entire sequence of the protein in question.

\subsubsection{Protein identification}

The negative control revealed no protein or any peptide, suggesting the method used for protein spot picking from both experiments was controlled.

MALDI-TOF-MS revealed spot 2 from experiment 1 to be Malate dehydrogenase, which was formely shown (Table 2.6) to increase by $30 \%$ in ID animals. Furthermore, spot 10 from experiment 2 was identified as guanine deaminase, which was shown to decrease by $25 \%$ in ID animals. Furthermore, from DIGE experiment 2, adenosine 5'-triphosphate (ATP) synthase $(\mathrm{H}+$ transporting mitochondrial F1 complex, beta subunit) (spots 11 and 12), nicotinamide adenine dinucleotide (NADH) dehydrogenase (ubiquinone Fe-S protein 1) (spot 9) and sirtuin 2 (silent mating type information regulation 2, homolog) (spot 2), were identified by MALDI-TOF-MS and formely found to be increased in ID animals, by $30 \%$, $30 \%$ and $20 \%$, respectively (Table 2.6 ).

Experiment 2, spot 2 resulted in an uncertain identification of sirtuin 2 (SIRT2) by MS/MS however was fully indentified by peptide mass fingerprinting (PMF), whereby a 44\% sequence coverage of the SIRT2 protein was shown (Table 2.7). When a protein had a Mascot score greater than 64, it was classed as significant in MS/MS $(P<0.05)$. However, as SIRT2 had a Mascot score of 63 meant this hit was recognised as uncertain under MS/MS. Furthermore, as this protein has many important and interesting functions as described in the introduction (section 1.2.4.1), will mean that out of the identified proteins SIRT2 will be further evaluated using quantitative Western blotting. This will help verify whether irondeficiency increases the expression of the SIRT2 protein in the midbrain and moreover, whether this increased expression exists in other regions of the CNS, i.e. the spinal cord. 


\begin{tabular}{|c|c|c|c|c|c|}
\hline $\begin{array}{c}\text { DIGE Experiment/ } \\
\text { Spot Number }\end{array}$ & $\begin{array}{c}\text { Mascot } \\
\text { Score }\end{array}$ & $\begin{array}{c}\text { Sequence } \\
\text { Coverage } \\
(\%)\end{array}$ & $\begin{array}{c}\text { Protein } \\
\text { Mass }\end{array}$ & pI & Identified Protein \\
\hline \multicolumn{6}{|l|}{ DIGE Experiment 1} \\
\hline 1 & - & - & - & - & undefined (no unequivocal peptide) \\
\hline $2 * *$ & 117 & 28 & 36659 & 6.16 & Malate dehydrogenase, cytoplasmic (Cytosolic malate dehydrogenase) \\
\hline DIGE Experiment 2 & & & & & \\
\hline 1 & - & - & - & - & undefined (no unequivocal peptide) \\
\hline $2 *$ & 63 & 44 & 29682 & 6.00 & sirtuin 2 (silent mating type information regulation 2, homolog) 2 (S. cerevisiae) \\
\hline 3 & - & - & - & - & undefined (no unequivocal peptide) \\
\hline 4 & - & - & - & - & undefined (no unequivocal peptide) \\
\hline 5 & - & - & - & - & Protein: undefined (no unequivocal peptide and unnamed protein) \\
\hline 6 & - & - & - & - & Protein: undefined (no unequivocal peptide and hypothetical protein) \\
\hline 7 & - & - & - & - & Protein: undefined (no unequivocal peptide and hypothetical protein) \\
\hline 8 & - & - & - & - & Protein: undefined (no unequivocal peptide) \\
\hline 9* & 61 & 23 & 74783 & 5.57 & NADH dehydrogenase (ubiquinone) Fe-S protein 1, isoform CRA_b \\
\hline $10 * *$ & 286 & 58 & 51494 & 5.36 & guanine deaminase \\
\hline $11 * *$ & 289 & 65 & 56265 & 5.19 & ATP synthase, $\mathrm{H}+$ transporting mitochondrial $\mathrm{F} 1$ complex, beta subunit \\
\hline $12 * *$ & 560 & 65 & 56265 & 5.19 & ATP synthase, $\mathrm{H}+$ transporting mitochondrial $\mathrm{F} 1$ complex, beta subunit \\
\hline
\end{tabular}

Table 2.7. MALDI-TOF-MS identified proteins. This table shows the identified proteins corresponding to spots picked from gels representing two independent 2-D DIGE experiments (Table 2.6), DIGE Experiment 1 and DIGE Experiment 2. Furthermore, the number of spots identified and subsequently evaluated by MALDI-TOF-MS varied between the two DIGE experiments. The Mascot score, protein mass, pI, the identified protein and its percentage sequence coverage of the identified peptides relating to the protein, are illustrated above. The dash (-) mark indicates that the presence of a protein was undefined (no unequivocal peptide). The asterisk (*) by the spot number indicates a protein was identified. One asterisk indicates partial recognition, where two marks complete identification by mass spectrometry (MS). 


\subsubsection{Quantitative Western blotting}

As this was the first time that SIRT2 had been identified with regards to iron-deficiency, meant further investigation of this protein would be of interest. In addition, myelin proteins were investigated as a variant of SIRT2 is colocalised in myelin sheaths. Therefore, the myelin markers, myelin oligodendrocyte glycoprotein (MOG) and PLP/DM20 were investigated. PLP/DM20 represents two splice isoforms of major CNS myelin proteins encoded by the gene PLP1 (Nave et al., 1987). In addition, differences in protein expression of the candidate markers PLP/DM20, DAT and TH were examined. Moreover, ChAT was also investigated. The identified protein expression of SIRT2, PLP/DM20, MOG, TH, DAT and ChAT, were correlated to an internal loading control, either Actin or glyceraldehyde 3-phosphate dehydrogenase (GAPDH). Observations of these proteins by Western blotting were made not only at the midbrain, but also at the spinal cord. Observing changes at the spinal cord meant findings would extend beyond the 2-D DIGE/MS investigated midbrain region. Use of quantitative Western blotting helped re-evaluate differences in protein expression which were previously identified in the 2-D DIGE/MALDI-TOF-MS experiments. Band densities from the Western blots were quantified using ImageJ 1.32j (NIH software, Bethesda, USA) giving absolute values, as described in the protocol (methods section 4.11).

The purpose of this study was to verify changes in SIRT2 and other proteins as described above. Due to the low sample number used in these experiments, meant no significant differences were observed in quantitative Western blotting, however the observed trends will be shown and discussed later.

\subsubsection{Mouse midbrain}

Western blotting revealed two different variants of SIRT2, a short v2 form located primarily in myelin and a longer v1 form located in neurons throughout the CNS. As previously observed in Western blotting, the different SIRT2 variants appear as two seperate bands with different molecular weights, 49kDa (v1) and 42kDa (v2) (Werner et al., 2007).

Quantitative Western blotting of SIRT2 in the midbrain was performed (Fig. 2.16a) using the same tissue samples as previously used for the DIGE experiments. The absolute mean grey (density) values of SIRT2 were calculated in relation to the house keeping gene GAPDH, therefore v1/gapdh and/or v2/gapdh, were determined (see Fig. 2.16f). SIRT2 v2/gapdh showed approximately $13.6 \%$ increase in ID WT animals compared to controls. This further supports the previous $20 \%$ increase in SIRT2 v2/gapdh identified by using 2-D DIGE (Fig. 2.16g) (see Table 2.6). The v1 variant was shown to remain relatively stable, as only a $3.6 \%$ increase was observed in ID WT mice. 
After determining the protein expression of SIRT2, the PLP/DM20 myelin marker was investigated (Fig. 2.16b), based on the specific localisation of SIRT2 v2 with PLP/DM20 within myelin sheaths (Werner et al., 2007). The myelin marker MOG was also investigated (Fig. 2.16b). The mean grey values from each band for MOG and PLP/DM20 were determined showing a $-4 \%$ and $-9 \%$ decrease respectively, in ID WT compared to control WT mice (see Fig. 2.16f).

In addition, candidate (DAT and $\mathrm{TH}$ ) and other markers (ChAT) were investigated in our ID mouse model (Fig. 2.16c-e), where trends were compared to previous findings in the literature. These results show a decrease $(-10 \%)$ in the expression of DAT in ID mice compared to controls. Moreover, TH expression was found to increase (22\%) and similarly with ChAT (9\%) in ID WT mice (Fig. 2.16f).
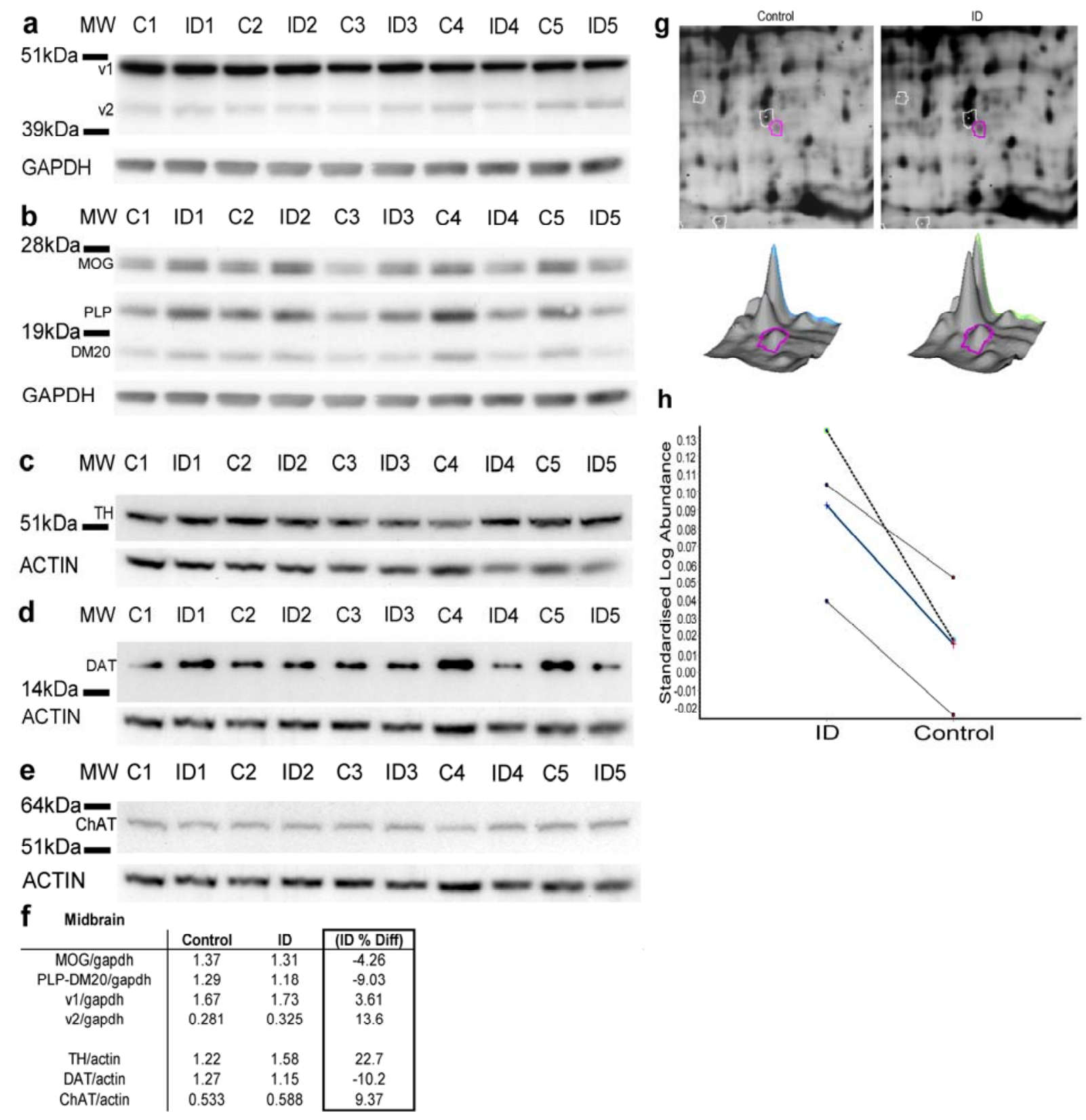

$\mathbf{h}$

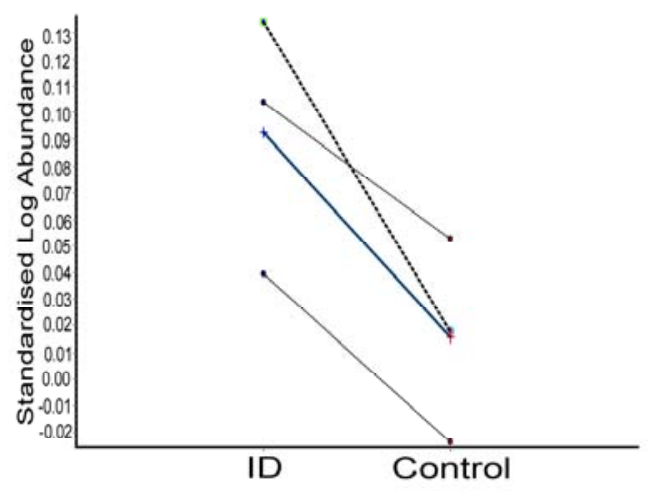


Fig. 2.16. Quantitative Western blotting of control WT and ID WT midbrain for SIRT2, myelin and candidate markers. a. SIRT2 protein was identified as two variants v1 and v2 in ID (ID1-ID5, $n=5$ ) and control $(\mathrm{C} 1-\mathrm{C} 5, n=5)$ mice. b. Myelin proteins MOG and PLP/DM20 were also evaluated in the same mouse groups, where GAPDH was used as an internal marker for both myelin and SIRT2 blots. c-e. For the proteins TH, DAT and ChAT, Actin was used as the loading control marker. MW represents the molecular weight (kDa) of the protein in question. f. Mean grey value (density) from Western blot analysis is shown in the table, where values correspond to internal markers (GAPDH, Actin). g. 2-D DIGE gel representation of the 20\% increase of SIRT2 (spot 2) identified in ID mice, highlighted by the purple line showing both two and three-dimensions of the spot. h. The standardised log abundance values (y-axis) of SIRT2 (spot 2) from the ID and control mice used in the 2D DIGE analysis. Blue line represents the ID and control mice which represent the group average where the corresponding 2-D DIGE image is shown $\mathbf{g}$.

\subsubsection{Mouse spinal cord}

Quantitative Western blotting of SIRT2 in the spinal cord was also performed, as shown in Fig. 2.17a, where absolute mean grey (density) values in relation to the house keeping gene GAPDH, therefore v1/gapdh and/or v2/gapdh were determined (see Fig. 2.17f). SIRT2 v2/gapdh showed an approximate 5\% decrease in ID WT mice compared to controls. In addition, the neuron localised SIRT2 variant, v1 showed a $15.5 \%$ decrease in ID WT mice in relation to GAPDH (v1/gapdh).

The PLP/DM20 myelin marker (Fig. 2.17b) and general myelin marker MOG were also investigated (Fig. 2.17b). The mean grey values from each MOG and PLP/DM20 band were determined. The results from the spinal cord were similar to that observed for ID WT midbrain tissue as described in the current study (Fig. 2.16f). This was a decrease in the expression of myelin specific proteins MOG (-11\%) and PLP/DM20 (-24\%) as described in Fig. 2.17f.

In addition, Western blotting was performed for the candidate markers (Fig. 2.17c-e). The results show a more stable expression of these proteins within the spinal cord where negligible changes were noted (Fig. 2.17f). These small changes in protein expression observed in ID mice compared to controls were, a decrease (-1\%) in the expression of ChAT and increases in DAT (5\%) and TH (1\%). 


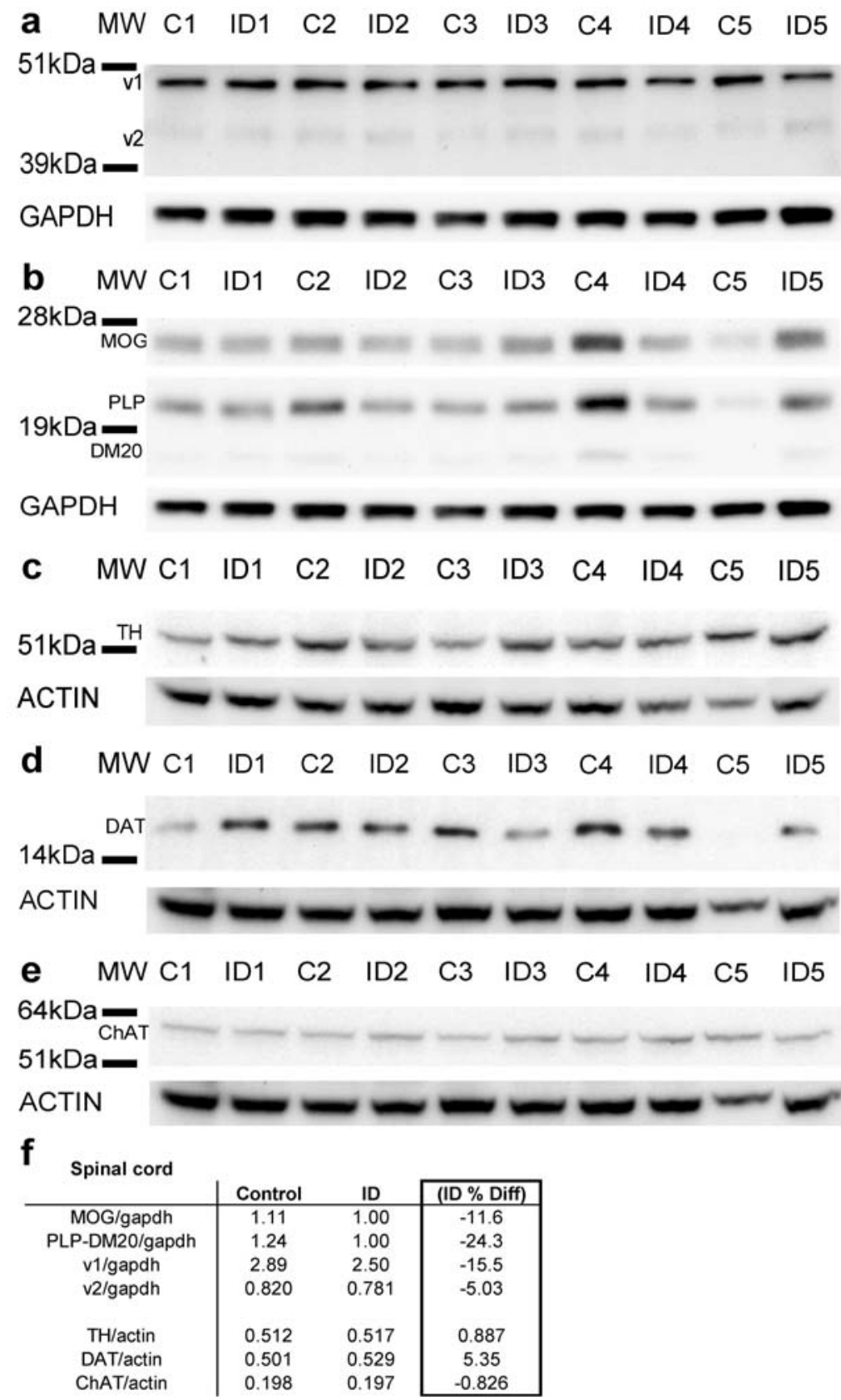

Fig. 2.17. Quantitative Western blotting of control WT and ID WT spinal cord for SIRT2, myelin and candidate markers. a. SIRT2 protein was identified as two variants v1 and v2 in ID (ID1-ID5, $n=5$ ) and control $(\mathrm{C} 1-\mathrm{C} 5, n=5)$ mice. b. Myelin proteins MOG and PLP/DM20 were also evaluated in the same mouse groups, where GAPDH was used as an internal marker for both myelin and SIRT2 blots. c-e. For the proteins TH, DAT and ChAT, Actin was used as the loading control marker. MW represents the molecular weight (kDa) of the protein in question. f. Mean grey value (density) from Western blot analysis is shown in the table, where values correspond to internal markers (GAPDH, Actin).

\subsubsection{Quantitative assessment of SIRT2 v2 variant in midbrain tissue from 2-D IEF/SDS-PAGE blots}

Using 2-D gel electrophoresis to separate proteins by isoelectric focussing (IEF) followed by a standard SDS-PAGE procedure (see methods section 4.12), proved useful in identifying the biochemical nature of both SIRT2 v1 and v2 variants. Several series of these 2-D IEF/SDSPAGE blots were prepared as an alternative to Western blotting to try and verify the increase in SIRT2 v2 observed in ID mice and to identify which isoforms making up the v2 variant 
show this increase (Fig. 2.18a). The myelin specific variant v2 forms the main focus of the analysis as myelin proteins have been previously investigated in the midbrain and spinal cord (section 2.6.3.). Due to the low sample number used in this series of experiments, the differences observed in these blots were not significant; however the trends shown will be discussed later. The same tissue samples as used for 2-D DIGE experiments were used here.

These 2-D IEF/SDS-PAGE blots confirm SIRT2 v2 to be increased in ID WT compared to control WT mice and that the majority of this increase is from isoform 2, where a $45.8 \%$ increase was observed in ID mice (Fig. 2.18c,d). The four different v2 and two v1 isoforms have previously been identified (Werner et al., 2007). The function of these isoforms is unknown, however it is believed they may possibly relate to different phosphorylation states of the variants (v1, v2). This was not further investigated in this thesis, however forms part of an ongoing study.

a

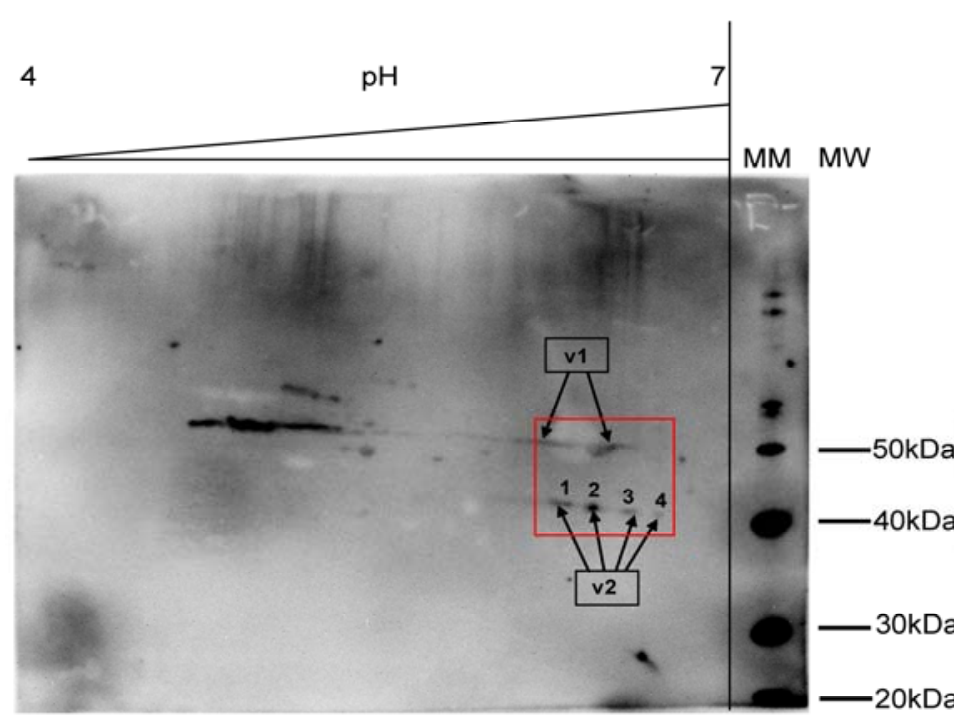

C

b

\begin{tabular}{cccccc|} 
Isoforms & $\mathbf{C 1}$ & $\mathbf{C 2}$ & $\mathbf{C 3}$ & $\mathbf{C 4}$ & \multicolumn{1}{c}{ Av } \\
\cline { 2 - 7 } $\mathbf{1}$ & 159.286 & 106.905 & 58.5 & 80.556 & 101.3118 \\
$\mathbf{2}$ & 169.175 & 165.75 & 0 & 64.333 & 99.8145 \\
$\mathbf{3}$ & 183.702 & 154.04 & 0 & 92.457 & 107.5498 \\
$\mathbf{4}$ & 192 & 170.625 & 74.5 & 0 & 109.2813 \\
\cline { 3 - 6 } Av & 176.0408 & 149.33 & 33.25 & 59.3365 & 104.4893 \\
\hline
\end{tabular}

\begin{tabular}{|c|c|c|c|c|c|}
\hline Isoforms & ID1 & ID2 & ID3 & ID4 & Av \\
\hline 1 & 45.969 & 143.333 & 132.551 & 60.883 & 95.684 \\
\hline 2 & 120.218 & 191.629 & 155.73 & 114.257 & 145.4585 \\
\hline 3 & 126.125 & 190 & 0 & 40.813 & 89.2345 \\
\hline 4 & 93.111 & 214.1 & 122.396 & 23.611 & 113.3045 \\
\hline$\underline{A v}$ & 96.35575 & 184.7655 & 102.6693 & 59.891 & 110.9204 \\
\hline
\end{tabular}
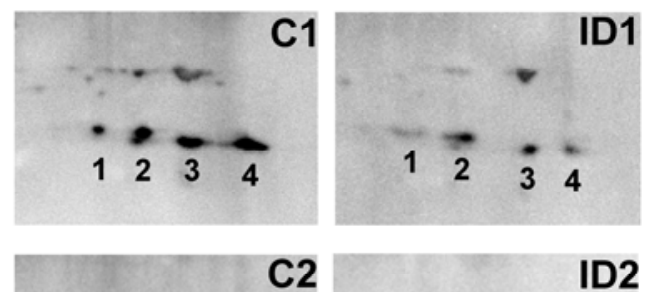

ID2

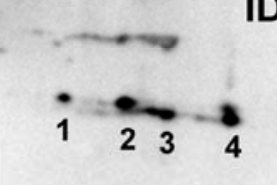

C3

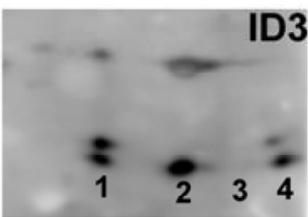

ID3
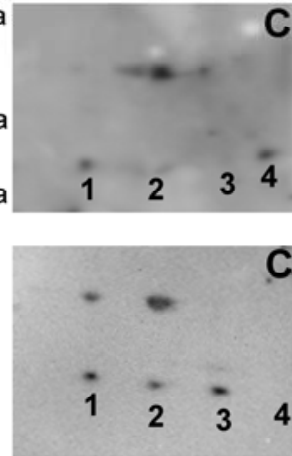

C4

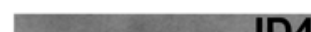

ID4

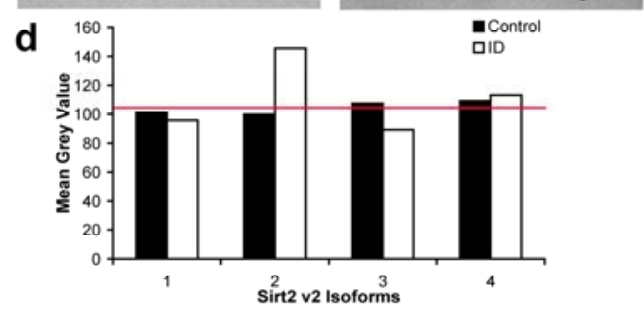

Fig. 2.18. 2-D IEF/SDS-PAGE blots of control and ID WT midbrain for SIRT2 v1 and v2 variants. a. Example of a 2-D IEF/SDS-PAGE blot, at a $\mathrm{pH}$ range from 4-7 (shown by top gradient bar) and varying molecular weight $(\mathrm{MW})(\mathrm{kDa})$ shown by the magic marker $(\mathrm{MM})$ on the right hand side gradient bar. Specific labelling of SIRT2 proteins (inside red box), identified v1 and v2 variants, where the latter is numbered 1-4. Each variant has specific states termed isoforms. b. Expanded region (red box) showing SIRT2 v1 and v2 isoforms clearly in control (C1-C4, $n=4)$ and ID (ID1-ID4, $n=4)$ mice. c-d. The Mean Grey values (density) of the 2-D IEF/SDS-PAGE blots are shown. b. The average $(\underline{\mathrm{Av}})$ mean grey value is given and plotted $\mathbf{d}$.The red line is representative of the maximum mean grey value for the majority of isoforms in both control $(\square, n=4)$ and ID ( $\square$, $n=4)$ groups. 
Chapter 3

Discussion 


\section{Discussion}

During the experimental part of this thesis 4 genetic variants involved in the pathophysiology of primary RLS were detected. Currently, 9\% of the population suffers from RLS. The most troublesome clinical cases are those with a familial background and a superimposed secondary cause. This thesis is focused on the most relevant secondary cause which is iron-deficiency. All methodological setups and concepts are easily transferable to genetic models when they are available.

The basic methodological findings of this thesis have shown the ID diet to induce a stable level of iron-deficiency with no severe anaemic effect. Essentially, this C57B1/6 mouse model of iron-deficiency exhibited several behavioural changes which include increased acute and chronic pain responses, and a heightened motor activity during late stages of the active dark phase in the nocturnal cycle of the mouse. An elevated motor activity was also observed in $\mathrm{D} 3^{-/-}$mice however at an earlier time period in the active dark phase. $\mathrm{D} 3^{-/-}$mice also showed heightened acute and persistent pain responses. At the spinal cord, ID WT mice show an increased cell activity at the dorsal horn and a possible increase in terminal innervation surrounding ventral motorneurons. A number of proteins were dysregulated at the midbrain region from ID mice. One of these identified proteins was found upregulated in the myelin. Further analysis identified a particular isoform to account for this increased expression of this myelin protein during iron-deficiency.

\subsection{Evaluating the effects an ID diet has upon the general physiology of WT mice}

The current study shows that ID diet does affect mice after varying durations of diet starting from P28, where initial changes were observed after 1 week. Establishing a mouse model of iron-deficiency was the essential prerequisite of this thesis, where subsequent testing relied on this evaluation. Therefore, it was important to identify the effects this ID diet had upon the general health of the mouse and whether long periods of ID diet administration caused severe anaemia. This was verified by measuring behavioural (grip strength, wheel-running performance) and physiological (haematology, weight) differences between ID and control mice, as will be discussed.

The aim was not to induce a strong anaemic effect in mice; rather a controlled level of iron-deficiency. Using a longer duration of ID diet would result in the ID animals becoming sick as caused by severe anaemia, however, 15 weeks was found to be an optimal duration of inducing iron-deficiency. 


\subsubsection{Influence of ID diet upon weight and muscle strength in WT mice}

In the current study, the average weight of ID mice was reduced after varying durations of ID diet. This effect has been previously identified in rats (Beard et al., 1995) and mice (KwikUribe et al., 1999).

The grip strength test was also used. The purpose of this test was to detect any change in neuromuscular integrity and muscle strength after prolonged durations of iron-deficiency. Previous studies found a decrease in grip strength when inducing iron-deficiency in offspring by administering mothers (dams) an ID diet (12.5-14 $\mu \mathrm{g}$ Fe/g diet) during gestation (KwikUribe et al., 1999; Kwik-Uribe et al., 2000b). In the current study, this test was used to check whether grip strength was altered when inducing iron-deficiency using our ID diet $(<8 \mathrm{mg}$ $\mathrm{Fe} / \mathrm{kg}$ diet) during post-natal stages. The result was no change in forelimb grip strength from wheel-running and non-wheel-running animals after 15 weeks of ID diet starting from age P28 (Burnes et al., 2008). This also applies for the hindlimbs as supported by the comparable (see Fig. 2.5c,d) wheel-running performance $\left(\mathrm{V}_{\max }\right)$ between ID and control mice during the dark phase. Therefore, ID and control groups have comparable musculoskeletal function based on the similar locomotive performance.

A separate study performed within our department (Klinker et al., in preparation) showed that, after 15 weeks ID diet administration no learning or memory differences were seen between control and ID WT mice. Memory differences were tested by using a complex wheel-running paradigm (unpublished data), where this test has previously been described as a effective method is determining memory differences in mice (Liebetanz and Merkler, 2006). A previous rodent study showed a reduced memory performance in offspring from dams administered an ID diet during gestation (Kwik-Uribe et al., 2000b). Overall, it appears that administering the ID diet during post-natal stages is a good option when wanting to induce a level of iron-deficiency which does not drastically affect the mouse. Therefore, it appears that the selected diet in combination with the $\mathrm{C} 57 \mathrm{Bl} / 6$ mouse strain results in a suitable approach for investigating the effects of iron-deficiency over prolonged durations.

\subsubsection{Influence of ID diet upon haematology in WT mice}

\subsubsection{ID diet reduces the plasma-iron concentration}

The plasma-iron concentrations after different ID diet durations indicate that once a certain decrease in the plasma-iron concentration is reached (after 4 week, see Fig. 2.1b), no further difference can be detected regardless of the duration of diet, which in the current study was at a 30 week maximum. At this time-point (30 week) both haematocrit and haemoglobin levels were decreased however within the normal range suggesting no sign of severe anaemia (Fig. 
2.1c,d). Previous studies show that ID diet reduces the plasma and/or serum iron concentrations when administering iron-deprived feed to $\mathrm{C} 57 \mathrm{Bl} / 6$ mice ( 4 and 8 weeks) (Qu et al., 2007). One possible reason for a lack of progressive decrease in plasma-iron concentration over prolonged periods of ID diet administration may be the remaining trace element of iron in the ID diet ( $<8 \mathrm{mg} \mathrm{Fe} / \mathrm{kg}$ feed).

A limitation of the present study which should be acknowledged is that iron-deficiency was determined by assessing iron levels in plasma and not by ferritin or transferrin levels within the blood plasma, cerebrospinal fluid, or brain tissue. Ferritin strongly correlates with iron and changes according to iron concentrations in the brain (Connor and Menzies, 1995). Previous studies have already shown brain and spinal cord iron concentrations to be significantly reduced in C57Bl/6 mice after 5 (Morse et al., 1999) and 8 weeks of ID diet (Qu et al., 2007; Zhao et al., 2007). Interestingly, the amount of ID diet-induced loss of brain iron has been shown to depend on mouse strain and sex, where $\mathrm{C} 57 \mathrm{Bl} / 6$ male mice appeared more sensitive to the effects of an ID diet compared to the DBA/2 male mice (Morse et al., 1999). This different sensitivity may contribute to the behavioural effects in our C57B1/6 mouse model of iron-deficiency.

Furthermore, with regards to the serum-iron levels it has been shown that after 8 weeks of ID diet in C57B1/6 mice the levels were reduced and that this reduction was correlated with a reduced brain and spinal cord iron concentration (Qu et al., 2007).

\subsubsection{ID diet alters haemoglobin and haematocrit}

Altogther the haemoglobin and haematocrit levels of ID mice are in the normal range (see Fig. 2.1c,d) as described in the results (section 2.2), however they differ on a group level (Beard et al., 2005; Dean et al., 2006).

Haematocrit is a measure of the erythrocyte volume in the blood and haemoglobin is an iron containing metalloprotein with function to transport oxygen in the blood (Cook and Skikne, 1989; Cook, 1992). Therefore, the significant decrease in haematocrit in ID mice compared to controls is caused by a decreased red blood cell volume in the blood as a result from the ID diet.

\subsection{The effect of iron-deficiency upon sensory behaviour in WT and $\mathrm{D3}^{-/-}$ mice}

Compared to the control mice, ID mice show increased pain responses after varying durations of ID diet. These increased pain responses were shown using a thermal (hot-plate test) and chemical (formalin-test) stimuli, representing acute and persistent inflammatory pain, 
respectively. At the varying durations of ID diet an increase in the persistent inflammatory induced pain was noted after 1 week of ID diet (Fig. 2.3b), however no change in the acute pain response was seen during this stage (Fig. 2.2a). In addition, ID WT mice also show increased acute and persistent inflammatory mediated pain responses after 4 and 15 weeks of ID diet (Fig. 2.2a and Fig. 2.3c,d). Moreover, eliminating the important antinociceptive D3 receptor resulted in a synergistic increase in persistent pain in ID $\mathrm{D}^{-/-}$mice compared to control D3 $3^{-/-}$mice (Fig. 2.3f). However, no such synergistic increase was found in ID D3 $3^{-/}$ mice during the acute pain response (Fig. 2.2b). Supporting immunohistochemistry work showed c-Fos expression to be upregulated in ID WT mice at superficial laminae I/II of the dorsal horn, ipsilateral to the side of formalin-injection (Fig. 2.4f).

D3 receptors are believed to play a central role in RLS. This is based from previous findings which have shown the following: (1) A specific D3 receptor antagonist (SB-277011) increases sleep latency compared to a D2/D3 receptor antagonist (sulpiride) (Austin et al., 2001). (2) Rotigotine, a preferential D3 receptor agonist (Scheller et al., 2009) is beneficial in treating RLS patients (Stiasny-Kolster et al., 2004a). (3) Preferential stimulation of D3 receptors in rodents reduces spontaneous locomotor activity (Svensson et al., 1994; Levant, 1997; Barik and de Beaurepaire, 2005), where conversely D3 receptor antagonists increase activity (Sautel et al., 1995; Clifford and Waddington, 1998). (4) D3 receptors are expressed in sensorimotor pathways (Suzuki et al., 1998; Levant and McCarson, 2001) and upon descending dopaminergic terminals of the spinal cord (Zhu et al., 2007). Also D3 receptor density is highest at the sensory dorsal horn which is also innervated by the diencephalic A11 region (Levant and McCarson, 2001), furthermore, this A11 region is believed to be a cause in generating abnormal sensations of the legs in RLS. (5) A greater activity observed in A11 lesioned mice was suppressed when using the D2/D3 agonist ropinirole (Zhao et al., 2007).

The aforementioned studies indicate the importance of the dopamine receptor system. A greater literature exists for D3 compared to D2 receptors in RLS. The latter receptor type is believed to play a role in the aetiology of RLS symptoms, however in the current study it was decided to observe the sensory and motor behavioural activity of $\mathrm{D} 3^{-/-}$mice for two main reasons. Firstly, D3 ${ }^{-/}$mice show a greater expression of D3 compared to D2 receptors at the lumbar spinal cord and are predominantly located at the sensory region (Levant and McCarson, 2001), moreover, D $2^{-/-}$mice have previously been investigated for pain responses (Mansikka et al., 2005). Secondly, $\mathrm{D}^{-/-}$mice show a level of motor activity which permits reliable assessment by the voluntary wheel-running test (see section 3.3 ), where $\mathrm{D} 2^{-/-}$mice are hypolocomotive and show an activity that is drastically reduced compared to WT mice (Baik 
et al., 1995). This suggests that knocking out the D2 receptor would result in an activity difference which would be difficult to quantify by the voluntary wheel-running test.

\subsubsection{ID WT mice show elevated acute and inflammatory pain responses assessed by the hot-plate and formalin tests}

In the current study, an increase in the acute pain response was shown after the ID diet durations of 4 and 15 weeks. A previous study (Yehuda and Youdim, 1984) found a similar non-significant trend after 4 weeks of ID diet in rats. One reason for this may be an interspecies difference to ID diet and to pain, where from personal experience mice show a greater sentitivity. Alternatively, the different hot-plate temperature used $\left(50^{\circ} \mathrm{C}\right.$ versus $\left.58^{\circ} \mathrm{C}\right)$ may account for the different results. Although a clear increase in the pain response was seen after 4 and 15 weeks in the present study (Fig. 2.2a), there was no change in the response time during the early stages of iron-deficiency (1 week). By observing the data, an increase in the acute pain response in ID WT mice seems dependent on the duration of ID diet.

Iron-deficiency results in a reduced dopaminergic output and DAT expression in the CNS, subsequently increasing synaptic pooling of dopamine (Beard et al., 1994; Nelson et al., 1997; Levenson et al., 2004). The increased acute pain response in ID mice might be caused by this decreased dopaminergic output and reuptake via DAT, causing increased synaptic dopamine. Another reason for the involvement of the dopaminergic system is that no noticeable increase in the acute pain response was observed until after 4 weeks of ID diet, suggesting a longer ID diet period may be required to induce a change in the dopaminergic system. The result of an increased synaptic dopamine may cause over stimulation of the pronociceptive D1 receptors. In addition, this receptor is expressed higher than any other dopamine receptor at the lumbar spinal cord (Zhu et al., 2007), where they are also upregulated in ID mice (Zhao et al., 2007).

In comparison to the hot-plate test which investigates the threshold for acute pain, the formalin-test is widely used to assess the prolonged pain component. The chemical formalin induces a biphasic response observed initially as an acute pain (Phase I) within the first 5 minutes of the test, which is followed by a persistent inflammatory pain (Phase II) lasting up to 60 minutes (Dubuisson and Dennis, 1977; Malmberg and Yaksh, 1992). Phase I and phase II are mediated by activation of the following primary afferent fibres, mixed $\mathrm{A} \delta$ - and C-fibres and C-fibres, respectively (Dickenson and Sullivan, 1987; Heapy, 1987; Ishizaki et al., 1999). In the present study, ID WT mice show an enhanced inflammatory pain (Phase II) compared to controls. Interestingly, this effect was present at all durations of iron-deficiency (1, 4 and 15 weeks). After recording the persistent pain response (0-45 minutes) mice were sacrificed at 50 
minutes, therefore representative of the final stages of the formalin-test whereby the active pain inducing effect of formalin was still present. Subsequently, c-Fos-IR was quantified indicating the nociceptive activity in the spinal cord after the formalin-test ( $\mathrm{Li}$ and Clark, 2001; Tao and Johns, 2002). An increased expression of c-Fos-IR cells was found at laminae I/II of the ipsilateral dorsal horn in ID WT mice only in the 1 and 4 week diet groups, where the 4 week group showed the greatest increase. This larger increase in c-Fos-IR cells after 4 weeks ID diet further supports the formalin-test pain data, as a greater pain response was seen during the final stages of the test (Fig. 2.3c). Therefore, this indicates that after 4 weeks of ID diet, an increased pain response is mediated by $\mathrm{C}$-fibre primary afferents.

D1 receptors have been shown to participate in late-phase long term potentiation (LTP) of C-fibre evoked potentials at the dorsal horn (Yang et al., 2005). Here, LTP is induced at synapses between afferent C-fibres and dorsal horn neurons (Liu and Sandkuhler, 1995) by stimulation of afferent C-fibres only and not A-fibres (Sandkuhler and Liu, 1998). The increased persistent pain response caused by iron-deficiency may involve LTP at synapses of primary afferent C-fibre terminals, which has been shown during the formalin test in rats (Sandkuhler and Liu, 1998; Ikeda et al., 2006). One possible piece of evidence linking the involvement of iron-deficiency to LTP of C-fibres is a previous study which identified an upregulation of D1 receptor mRNA and protein in the lumbar spinal cord of ID mice (Zhao et al., 2007). From this, one may speculate that increased formalin-induced pain observed in our ID mice may be a result of enhanced D1 receptor mediated LTP. Moreover, sensory symptoms in iron-deficient RLS patients may be linked to altered LTP at C-fibre terminals.

In relation to previous clinical studies which identified a change in $\mathrm{A} \delta$ - and $\mathrm{C}$-fibre function in RLS patients (Schattschneider et al., 2004; Stiasny-Kolster et al., 2004b), the hot-plate test in rodents assesses pain transmission through A $\delta$-fibres (Frolich et al., 2005) and the formalintest through mainly C-fibres (Dubuisson and Dennis, 1977; Yaksh et al., 1999). Therefore, our results suggest that iron-deficiency may cause both an alteration of $\mathrm{C}$ - and $\mathrm{A} \delta$-fibre mediated pain perception in iron-deficient RLS patients. C- and A $\delta$-fibres innervate the dorsal horn (Shepherd, 1994), a region where a higher c-Fos expression was identified (Fig. 2.4f).

\subsubsection{Increase in acute pain response in $\mathrm{D3}^{-/-}$mice and a synergistic increase in prolonged inflammatory pain induced by iron-deficiency}

The acute pain response was significantly greater for $\mathrm{D} 3^{-/-}$compared to WT mice (Fig. 2.2b). However no further increase $(P>0.05)$ in the acute pain response was observed in ID $\mathrm{D}^{-/-}$ compared to control $\mathrm{D} 3^{-/-}$mice. This suggests that $\mathrm{D}^{-/-}$mice are more sensitive to acute pain 
which is irrespective of iron-deficiency. A possible reason for this is that in order for ID to produce a reduced acute pain response the $\mathrm{D} 3$ receptor must be present.

The persistent pain response was greater in $\mathrm{D}^{-/-}$compared to WT. However, in contrast to the acute pain response, iron-deficiency was shown to synergistically increase the persistent pain response only in $\mathrm{D}^{-/-}$mice during phase II of the formalin-test. This suggests that the presence of D3 receptors is involved in regulating persistent inflammatory pain. However, when D3 receptors are absent, iron-deficiency is still shown to have a role in enhancing the pain response. This suggests that iron-deficiency must induce this pain enhancing effect through an alternative pathway to the dopaminergic receptor system, where all receptors have been reported to be expressed in the dorsal horn region of the lumbar spinal cord (Zhu et al., 2007). One possible reason may be due to the involvement of a different neurotransmitter system, for example the glutamatergic system, as discussed below (section 3.2.2.1.).

To date there are no studies which have investigated acute and persistent pain response differences in $\mathrm{D}^{-/-}$mice by using the hot-plate and formalin tests. However, one study in $\mathrm{D}^{-/-}$ mice reports an increased spinal cord excitability (Clemens and Hochman, 2004), in particular a facilitation of spinal cord reflexes in the presence of dopamine (Clemens et al., 2006). This may explain why $\mathrm{D}^{-/-}$mice show greater acute and persistent inflammatory pain responses. In addition, as D3 receptors are present upon primary afferent $\mathrm{A} \delta$ - and $\mathrm{C}$-fibres at laminae $\mathrm{I} / \mathrm{II}$ of the spinal cord (Xie et al., 1998) (see Fig. 1.1) and regulate the function of postsynaptic D1 and D2 receptors (Xu et al., 1997; Wong et al., 2003), suggests the participating role of the dopaminergic system and dopamine receptors as a cause of sensory RLS symptoms, which will be discussed below.

\subsubsection{Involvement of dopamine and possibly glutamate receptors in enhancing acute and} persistent pain responses in $D 3^{-/}$mice

The pain symptoms associated with RLS are known to involve descending monoaminergic pathways (see Fig. 1.1), as highlighted in a review (Benarroch, 2008). Current literature shows a strong connection between iron, pain perception and the dopaminergic system. Moreover, dopamine agonists have been clinically shown to have a beneficial effect when used in the treatment of both primary and secondary RLS (Akpinar, 1982; Earley and Allen, 1996) and are considered as first-line therapy. However, other neurotransmitters and their receptors may be the cause of the increased pain response in ID $\mathrm{D}^{-/-}$mice during phase II of the formalin test. This seems plausible as there are many different receptor types expressed in laminae I/II 
of the dorsal horn (see Fig. 1.2) as will be discussed below in relation to the acute and persistent pain findings of the current study.

Neuroanatomically, primary C-fibre afferents mostly innervate lamina II interneurons, which in turn mediate nociceptive information to dorsal horn projecting neurons residing in lamina I (Nestler, 2001). Primary afferent A $\delta$-fibres directly innervate dorsal horn projecting neurons (Nestler, 2001), where D3 receptors are known to reside upon these lamina I A $\delta$-fibres (Xie et al., 1998). Therefore, this may explain why mice lacking the D3 receptor show increased acute pain responses. Moreover, this increased acute and persistent pain responses in $\mathrm{D} 3^{-/-}$mice is mainly caused by eliminating the antinociceptive function of D3 receptors (Levant and McCarson, 2001). D3 receptors also regulate dopamine release at presynaptic terminals and in combination with D2 modulate the function of pronociceptive D1 receptors (Mizuo et al., 2004; Le Foll et al., 2005). Therefore, in the absence of D3 receptors, D1 receptors may go unchecked and become overactive. This may also be a reason for the enhanced C-fibre mediated activity in $\mathrm{D}^{-/-}$mice. In addition, D3 receptors work in combination with antinociceptive D2 receptors to inhibit the influx of intracellular calcium through channels upon primary afferent fibres (Levant and McCarson, 2001; Millan, 2002), where subsequently the neurotransmitter glutamate and the modulator substance P (SP) are released (Levant and McCarson, 2001; Benarroch, 2008) from laminae II primary afferent C-fibres. The release of glutamate produces a rapid response mediated by the activation of both NMDA and $\alpha$-amino3-hydroxyl-5-methyl-4-isoxazole-propionate (AMPA) receptors residing upon dorsal horn neurons (Nestler, 2001). A reason for the increased pain response in $\mathrm{D} 3^{-/-}$mice may be from primary afferent $\mathrm{C}$ - and A $\delta$-fibres causing an excitatory postsynaptic potential (EPSP) mediated by glutamate release, which subsequently activates AMPA and NMDA receptors (Nestler, 2001). Therefore, the synergistic increase in formalin-induced C-fibre mediated pain in ID D3 ${ }^{-/}$mice might be caused by a simulatenous activation of not only glutamate receptors but also increased D1 and reduced D2 receptor function at dorsal horn projection neurons (Ben-Sreti et al., 1983; Zarrindast and Moghaddampour, 1989; Liu et al., 1992; Zarrindast et al., 1999). The involvement of NMDA receptors seems plausible based on previous work which showed that once these receptors are blocked C-fibre activation is prevented (Liu and Sandkuhler, 1995).

\subsection{The effect of iron-deficiency upon the pattern of circadian motor activity}

The human phenotypes of RLS are commonly described as restlessness and periodic limb movements with an urge to move the affected limbs primarily before sleep. The voluntary 
wheel-running test was used to determine whether similar phenotypes arise in our ID mouse model.

Firstly, the voluntary wheel-running system (Liebetanz and Merkler, 2006; Liebetanz et al., 2007) helped investigate whether the ID diet was altering the mouse's activity at specific periods during the dark phase i.e. prior to the start of the light phase, which is when symptoms commonly prevail in humans. The parameters used to detect these activity changes were termed "activity parameters" and include: (1) the activity time $\left(\mathrm{T}_{\text {run }}\right)$; (2) number of runs $\left(\mathrm{N}_{\text {run }}\right)$ and; (3) the percentage of the activity time in comparison to the maximum achieved during the the 19:00hr-03:00hr period of the dark phase (Percentage max of $\mathrm{T}_{\text {run }}$ ) and; (4) percentage of number of runs (Percentage max of $\mathrm{N}_{\mathrm{run}}$ ) in comparison to the maximum achieved during the same 19:00hr-03:00hr period of the dark phase. These parameters proved important, particularly Percentage max of $\mathrm{N}_{\text {run }}$ and Percentage max of $\mathrm{T}_{\text {run }}$, as they closely resemble the human RLS symptoms of restlessness and increased activity, respectively. These parameters were shown to be increased by iron-deficiency in WT and D3 $3^{-/-}$mouse strains, as will be discussed (section 3.3.1).

The second set of parameters is a measure of endurance and was used as a control to the activity (described above) by detecting whether iron-deficiency per se was having any relevant effect in the mouse's overall ability to run on the running wheel, particularly at the important stages i.e. prior to the start of the light phase. The parameters used for detecting these endurance differences were termed "performance parameters" and included Distance, $\mathrm{V}_{\max }$ and Distance/ $\mathrm{N}_{\text {run }}$, as will be discussed (section 3.3.2). These parameters are commonly associated with the endurance and skill of an animal during wheel-running experiments (Billat et al., 2005; Liebetanz et al., 2007).

\subsubsection{Iron-deficiency elevates voluntary wheel-running activity in WT and $\mathrm{D3}^{-/-}$mice}

ID WT mice show an increased activity during the latter hours of the dark phase (Fig. 2.11), while the performance remained unchanged (Fig. 2.5b,d,f). This was shown by the following parameters which relate to the running activity of the mouse: Percentage of max $\mathrm{N}_{\text {run }}$ and Percentage of max $T_{\text {run. }}$. These activity parameters were increased after 15 weeks diet in ID WT mice at a 2 hour time period before the start of the light phase. This increased activity occurs at a time-point which corresponds to the late evening period in humans, which is when RLS symptoms commonly arise (Walters, 1995).

After both 4 and 15 weeks of diet, ID D3 ${ }^{-/-}$mice showed an increased activity during the latter third of the dark phase (03:00hr-07:00hr) (Fig. 2.11). This heightened activity in ID $\mathrm{D}^{-/-}$mice is seen at least two hours earlier when compared to ID WT mice. This shift of heightened motor activity to an earlier time might be compared to augmentation in RLS, 
which is typically shown by a 2-4 hour advance of RLS motor symptoms from late evening to an earlier time-point in the day (Allen et al., 2003; Garcia-Borreguero et al., 2007). Typically, augmentation is shown in patients being treated with dopaminergic drugs (Allen and Earley, 1996); however, it is still unknown whether the clinical deterioration of symptoms in drugnaive RLS patients by iron-deficiency can be compared to augmentation, which by definition, is a paradoxical worsening of RLS symptoms by drug overdosage. If the D1 receptor shift hypothesis of Paulus and Trenkwalder (2006) for augmentation can be generalised to iron deficiency (Paulus and Trenkwalder, 2006) which also may selectively reduce D2 receptors (Ashkenazi et al., 1982; Youdim et al., 1986; Beard et al., 1993), a comparision can be made.

In contrast, activity was significantly decreased for ID WT and ID D3 ${ }^{-/-}$mice during the early hours of the dark phase. One interesting point which must be addressed is that at specific time periods corresponding to when human RLS symptoms arise, ID WT and ID D3 $3^{-/}$ mice show greater wheel-running activity at a level comparable to that observed at the start of the dark cycle (19:00hr-22:00hr). This suggests that ID WT and ID D3/-- mice voluntarily run at this heightened level because of the effects of iron-deficiency, as will be discussed (section 3.3.1.1).

In the literature there are many behavioural studies performed on ID rodents which describe changes in activity, however they show mixed results. An early study in rats showed irondeficiency to induce a complete reversal of the nocturnal circadian cycle, resulting in a greater activity during the light phase (Youdim et al., 1981). This reversed activity was not seen in the current study in ID mice (Dowling et al., unpublished data) nor in a previous ID rodent study (Hunt et al., 1994). Another study also showed an increase in locomotor activity in ID WT mice after lesioning the diencephalospinal A11 region with 6-OHDA. However, the increased activity was observed only at the beginning of the dark phase, which is at a non-comparable time period to when symptoms arise in humans (Zhao et al., 2007). Only one study to date has shown iron-deficiency to increase restlessness at a period before the start of the rest phase in rodents, matching when symptoms occur in humans. However, what was identified was not an increase in motor activity but an elevated wakefulness, measured by EEG 4 hours before the rest phase (Dean et al., 2006). The current findings of this thesis confirm an increased motor activity and in addition showed the activity time to increase which can be compared to a state of wakefulness or restlessness in the animal. In addition, using the voluntary wheel-running behavioural test resulted in clear increases in activity time and the number of runs during important time periods which correspond to when motor symptoms arise in humans. 


\subsubsection{Involvement of iron-deficiency and the dopaminergic system}

The iron-deficiency induced increase in activity of ID WT and ID $\mathrm{D} 3^{-/-}$mice appears under circadian influence. This suggests that normal iron levels are a contributing factor in regulating activity. One reason how iron regulates this circadian onset of symptoms during the dark phase, may be due to the fluctuating nature of iron levels between light and dark phases (Unger et al., 2008). Unger and colleagues (2008) showed that compared to the light phase peripheral blood levels of iron decrease during the dark phase in male mice, where a greater decrease was found in ID compared to control mice (Unger et al., 2008). This fluctuating iron level may be a cause of the circadian onset of RLS symptoms. Furthermore, as iron-deficiency is believed to decrease dopamine production by reducing activity of the dopamine synthesising enzyme TH (Youdim and Green, 1978), one can predict a reduced dopamine drive from the diencephalospinal A11 brain region to the spinal cord in mice. This A11 region is important in controlling normal locomotor activity because when lesioned in mice an increased locomotor activity time was seen which was further increased during iron-deficiency (Zhao et al., 2007). Reducing dopaminergic output from this A11 region may result in an increased spinal excitability, as dopamine functions in decreasing spinal excitability (Clemens et al., 2005). Moreover, this explains why in the current study ID WT mice show increased motor activity time. Again this correlates to the clinical benefits of using dopamine agonists in treating RLS symptoms (Akpinar, 1982; Earley and Allen, 1996) and in restoring the increased activity in the lesion study described above (Zhao et al., 2007).

ID D $^{-/-}$mice show a unique time period of increased motor activity, which prevails 2-4 hours earlier than the increased motor activity in ID WT mice. This shifting effect of increased activity in ID $\mathrm{D}^{-/-}$mice to an earlier time period can be described by two factors. Firstly, iron is at a low concentration during the dark phase in mice which is even less in ID male mice (Unger et al., 2008). Secondly, the time period showing an elevated activity in ID D3 ${ }^{-/}$mice, approximately $03: 00 \mathrm{hr}$ corresponding to late afternoon in humans, coincides with the point of lowest dopamine concentration in mice (Huie et al., 1989; Khaldy et al., 2002). Therefore, a reason for this increased activity in ID $\mathrm{D}^{-/-}$mice at this time period is likely caused by a suppressed dopamine output which is caused by the synergism of iron-deficiency in reducing dopamine production by decreasing the function of $\mathrm{TH}$ combined with the already low dopamine levels during this time-period. From this one would expect this low circulating level of dopamine to cause increased spinal cord excitability (Clemens et al., 2005). This is supported by a previous study by Zhao and colleagues (2007) that showed an increase in locomotor activity after the A11 region was lesioned (Zhao et al., 2007). In addition, the same 
study showed a significant decrease in D2/D3 receptor protein expression at the lumbar spinal cord. Also the increased motor activity shown was normalised after administration of the D2/D3 agonist ropinirole (Zhao et al., 2007). This study by Zhao et al., (2007) shows not only the importance of dopaminergic output from the A11 region in controlling motor activity, but also the function D2 and D3 receptors may play in the spinal cord in preventing increased motor activity. This explains why an increase in motor activity is seen in ID $\mathrm{D}^{-/}$mice. Moreover, as the D3 receptor has an important function in maintaining the activity of both D1 and D2 receptors, (Carr et al., 2002; Wong et al., 2003) suggests the D1 and D2 receptors are no longer modulated in $\mathrm{D}^{-/-}$mice. In addition, the D3 receptor has been shown to inhibit spontaneous locomotion (Sautel et al., 1995; Mizuo et al., 2004), where central D1 and D2 receptors have been implicated in controlling locomotor activity (Barriere et al., 2004). This suggests that in the absence of D3 receptors a change in locomotor control may occur, which may be influenced wholly by an increase in D1 receptors which possess a known function in recruiting a locomotor pattern (Zhu et al., 2007). This seems plausible especially as an increase in D1 receptor protein was found in the lumbar spinal cord of ID WT mice (Zhao et al., 2007). Moreover, it has also been proposed that an increased activity of D1 receptors may play a role in sleep wake arousal and restlessness (Qu et al., 2008).

The increase in motor activity in ID $\mathrm{D}^{-/-}$mice is most likely caused by a preferential increase in activation of D1 receptors over D2 receptors at the lumbar spinal cord, where both these receptors are normally maintained by the D3 receptor. Also iron-deficiency further reduces the dopaminergic output via the diencephalospinal A11 region resulting in increased wakefulness and restlessness. Therefore, iron-deficiency is a key factor in inducing the increased activity seen in ID WT and ID $\mathrm{D}^{-/-}$mice. This increase in activity is likely related to the sensation of restlessness, because both wakefulness and restlessness are modulated by the dopaminergic system (Rye, 2004), which is at its lowest level at the period coinciding with the increased activity detected by the voluntary wheel-running test.

Finally, as D3 receptors are important in sensor motor integration because of their distribution throughout dorsal/ventral horns and pars centralis (Levant and McCarson, 2001), suggests why an increase in pain response was also found in addition to increased running activity in ID D3 ${ }^{-/}$mice within the current study (Dowling et al., unpublished data).

\subsubsection{Chronic administration of ID diet has little suppressive effect upon wheel-running performance in $\mathrm{WT}$ and $\mathrm{D3}^{-/-}$mice}

In addition to the activity measurements $\left(\mathrm{N}_{\text {run }}, \mathrm{T}_{\text {run }}\right.$, Percentage of max $\mathrm{N}_{\text {run }}$, Percentage of max $\mathrm{T}_{\text {run }}$ ) Distance, $\mathrm{V}_{\max }$ and Distance/ $\mathrm{N}_{\text {run }}$ were measured as a control of the activity between the different diet groups. One observation was that ID WT and ID D3 $3^{-/-}$mice show a decrease in 
wheel-running Distance during the beginning of the active dark phase. Iron-deficiency is most likely a reason for this reduced performance, because of a similar effect in both strains. Importantly, the physical running ability of mice as measured by $\mathrm{V}_{\max }$, was very stable between all groups for WT and $\mathrm{D}^{-/-}$mice. This indicates that the ID diet was having no detrimental effect upon the mouse's performance ability on the running wheel, even after prolonged durations of diet.

In comparison to ID WT mice, ID D3 ${ }^{-/-}$mice also show a further decrease in wheelrunning performance, Distance and Distance/ $\mathrm{N}_{\text {run. }}$. The cause of this effect must be mediated by iron-deficiency in synergism with $\mathrm{D} 3^{-/-}$, particularly as control $\mathrm{D} 3^{-/-}$mice were shown to have a greater running performance compared to WT mice, which is mediated by an increased basal dopamine release (Xu et al., 1997) because D3 receptors function in inhibiting dopamine release (Tang et al., 1994). Therefore, iron-deficiency has a greater effect in reducing dopamine levels in $\mathrm{D}^{-/-}$compared to WT mice. Most likely this occurs by iron-deficiency reducing the function of the iron-dependent dopamine synthesising enzyme TH (Youdim and Green, 1976), which is also known to be reduced in D3 $3^{-/-}$mice (Le Foll et al., 2005) and also how iron-deficiency decreases the expression of DAT (Erikson et al., 2000).

\subsubsection{Iron-deficiency increases innervation of ChAT-IR terminals upon ventral motoneurons}

The presence of $\mathrm{ACh}$ in spinal motoneurons is important in eliciting a spontaneous motor activity. Also choline acetyltransferase (ChAT) is important in formation of mammalian neuromuscular synapses (Brandon et al., 2003), where ChAT mutant mice demonstrate a reduced level of activity and abnormal regulation of flexor-extensor and left-right alternation of hindlimbs (Myers et al., 2005). In addition, ChAT is important in producing spontaneous activity at early stages of embryonic spinal cord development (Myers et al., 2005). These experiments were performed to identify whether ventral motoneurons at a region known to be important in movement initiation and locomotor pattern generation (Kiehn, 2006), change in WT mice after voluntary wheel-running at 4 and 15 week ID diet durations.

After completing either a 4 or 15 week voluntary wheel-running experiment in WT mice, immunohistochemistry was performed on thoracolumbar ventral motoneurons. Motoneurons at the entire ventral horn region of the thoracolumbar (T13-L2) spinal segments were investigated for changes in area and ChAT-IR terminal innervation. The result was an increased number of ChAT-IR terminals in ID WT mice only after 15 weeks of ID diet, where the motoneuron area did not change between ID WT and control WT mice after both 4 and 15 week ID diet durations. 
The increase in terminal innervation was located upon motoneurons of laminae VIII and IX (see Fig. 1.3). These motoneurons are located proximal to ascending and descending long-range and short-range interneurons which make up the CPG network required in leftright alternation and flexor-extensor coordination (Kiehn and Butt, 2003). Also, it has been shown that glutamatergic V3 neurons innervate motoneurons at laminae VIII and locomotor related interneurons particularly parvalbumin-positive Ia-INs and calbindin-positive renshaw cells (RCs) (Zhang et al., 2008). Furthermore, RCs and Ia-INs are spinal inhibitory interneurons projecting to motoneurons and function in flexor-extensor coordination and with the CPG in fine tuning motoneuronal firing and burst termination, where Ia-INs also function in inhibiting rhythmic activity of motoneurons initiated during locomotion. Therefore, the increased terminal innervation may arise from RCs and Ia-INs in an attempt to compensate for an abnormal motoneuron firing pattern. Other reasons for the increase in terminal innervation may be linked to an increase in L2 descending commissural interneurons (dCINs) attempting to inhibit contralateral flexor motoneuron activity through a glycinergic pathway (Kiehn, 2006), or via ascending commissural interneurons (aCINs), where a small subset are cholinergic and show a greater localisation between T13 and L2 segments (Juvin et al., 2005). Overall, the increase in ChAT-IR terminals upon ventral motoneurons at this region (area 2, see Fig. 2.14e) may be caused by an increased number of the aforementioned interneuron contacts which are in close proximity. Therefore, this terminal increase may relate to a change in the CPG, where at this region it is important in controlling hindlimb movements as described in rodents (Nishimaru et al., 2000; Kiehn and Butt, 2003). In addition, it will be of interest to investigate receptors for the neurotransmitters, NMDA, DA and 5-HT which may be involved, as they have all been previously shown to induce locomotion at the T11-L2 region, in particular 5-HT (Schmidt and Jordan, 2000).

The area of autonomic IML motoneurons located at lamina VII did not change between diet groups or after different ID diet durations. This region was investigated because diencephalic A11-A14 neuron groups project to this spinal region (Kalen et al., 1988), where the A11 region is believed to be involved in RLS (Clemens et al., 2006). After finding a change in ChAT-IR terminal density at laminae VIII/IX ventral motoneurons, it seemed of interest to evaluate whether ChAT-IR motoneurons at the IML, a region which may be involved in RLS symptoms, showed any sign of change under iron-deficiency.

The increased innervation of ChAT-IR terminals might be an underlying cause for the increased voluntary wheel-running activity observed in ID WT mice after 15 weeks diet, as the investigated region is known to be important in regulating movement initiation. However, further experiments are needed to confirm this. 


\subsection{Influence iron-deficiency has upon protein expression in the CNS}

\subsubsection{Identified proteins}

The purpose of this study was to perform a screen for proteins which may show dysregulation during a state of iron-deficiency. The brain region selected for protein screening contained all dopaminergic cell bodies, including the diencephalic A11 region (Paxinos, 2003; Qu et al., 2007) which is an important bilateral dopamine rich group of neurons providing the only source of dopaminergic neurotransmission into the spinal cord (Lindvall et al., 1983; Skagerberg and Lindvall, 1985). In addition, the SCN was also included in this region as it is an important structure involved in the circadian control of the nervous system (Moore, 1996) and forms connections with the A11 region (Reuss, 1996).

The following proteins were fully identified in the current study, malate dehydrogenase, guanine deaminase and ATP synthase. Finally, NADH dehydrogenase and SIRT2 were partially recognised using MS/MS, although they were fully identified using PMF. From these proteins, SIRT2 was followed up by further analysis as this protein was of interest. The increased expression of SIRT2 in ID WT mice was further verified by implementing other techniques from which these results will also be discussed.

A previous study utilised 2-D Gel electrophoresis to determine changes in the expression of midbrain proteins after inducing iron-deficiency in-vivo. After 5 weeks of inducing iron deficiency in rats starting at P21, Youdim and colleagues (1986) described 150 target proteins to be altered during iron-deficiency in the caudate nucleus and NAcc (Youdim et al., 1986). However, only 10 were significantly affected in iron-deficiency in the caudate nucleus. Those that were identified were glial fibrillary acidic protein (GFAP) and albumin, which in ID rats were significantly decreased (-38\%) and increased (46\%), respectively. In this study, iron-deficiency also altered the expression of neuron-specific enolase (NSE) which was increased by $22 \%$ in the NAcc from ID rats (Youdim et al., 1986).

A reason for the difference in the previous study compared to that of the current study is that the proteins were not fully identified by modern-day MS. Instead proteins were identified by their molecular weight from the 2-D gel. This method is now considered not as accurate and therefore very different from the current study which used MALDI-TOF-MS. Other reasons which may account for the differences are, a different rodent genus was used, the ID diet was commenced at P21 instead of P28 and finally the ID diet was continued for only 5 as opposed to 15 weeks. 


\subsubsection{SIRT2 and myelin markers}

Due to the low sample number used in these experiments meant the differences observed from the quantitative Western blots and 2-D IEF/SDS-PAGE blots were not significant. However, the observed trends from these experiments were similar and will be discussed in relation to the identified SIRT2 protein by MALDI-TOF-MS.

This study revealed the possible connection between SIRT2 and iron-deficiency. Initially, 2-D DIGE experiments found a $20 \%$ increase in SIRT2 within ID WT mice. Subsequent quantitative Western blotting identified an approximate 14\% increase in SIRT2 to come from the short variant (v2) predominantly found in myelin. Further confirmation of this increased level of SIRT2 v2 was achieved by 2-D IEF/SDS-PAGE (see Fig. 2.18), where an increase in v2 isoform number 2 was present in ID WT mice. Western blotting further revealed the myelin marker PLP/DM20 and MOG in relation to the house keeping gene gapdh, to decrease in ID WT mice. This data trend suggests that within the selected tissue region (entire midbrain) the level of SIRT2 v2 may be elevated during a state of reduced myelin. A reduced level of myelin was also found in the spinal cord, however the expression of SIRT2 v1 and v2 was decreased. The presence of reduced myelin proteins PLP/DM20 and MOG within the brain and spinal cord in ID animals is most likely as a result of iron-deficiency, as described in previous ID rodent studies (Yu et al., 1986; Kwik-Uribe et al., 2000a; Erikson et al., 2001). Furthermore, other studies also confirm that inducing iron-deficiency, whether at pre- or post-weaning stages results in a hypomyelinated state (Beard et al., 2003b). The decrease in myelin is most likely caused by the change in the levels of iron, Tf and ferritin in oligodendrocytes (Connor, 1994), which are important for myelination (Kwik-Uribe et al., 2000a).

One interesting point is how an increase in SIRT2 v2 may occur during a state of hypomyelination at the midbrain. A previous study showed the presence of PLP/DM20 is required for SIRT2 v2 to be transported into the myelin (Werner et al., 2007). Therefore, one would expect a decrease in SIRT2 v2 as PLP/DM20 was also decreased in the midbrain. However compared to the midbrain, a decrease in SIRT2 v2 and PLP/DM20 was confirmed at the spinal cord, therefore why should the midbrain be any different? One reason is that the brain compared to the spinal cord has a higher concentration of iron because it has a higher oxidative metabolism required for neuronal function (Yehuda, 1988; Cho et al., 1998; Siegel, 1999). Therefore, during iron-deficiency the spinal cord is greater affected because it does not have a higher iron reserve pool. Also as iron has an important role in myelination (Yu et al., 1986; Connor and Menzies, 1990; Ward et al., 2007), a greater reduction in PLP/DM20 and 
MOG myelin proteins was shown at the spinal cord. Therefore, a reason for the SIRT2 v2 decrease in the spinal cord is because of this greater reduction in PLP/DM20 which is colocalised with SIRT2 v2 in myelin sheaths. However, the reason for the increase in SIRT2 $\mathrm{v} 2$ in the midbrain during iron-deficiency may be explained by the greater presence of iron from reserve pools in the midbrain, resulting in a less dramatic decrease in myelin proteins and myelinogenesis, therefore a greater quantity of PLP/DM20 which will stablise SIRT2 v2, as previously suggested (Werner et al., 2007). Therefore iron regulates SIRT2 v2 through its influence upon myelin production. However, the increase may also arise from an unknown function of SIRT2, possibly as an iron-sensing protein in the myelin sheath, or SIRT2 overexpression may be associated with diminished maturation of oligodendrocytes, notably tubulin acetylation and arborisation, as previously shown (Fulco et al., 2003; Li et al., 2007).

In addition, animal models have proposed an elevated SIRT2 expression in Alzheimer's and Huntington's diseases (Green et al., 2008; Pallos et al., 2008). These diseases have also been described in connection with iron-deficiency (Youdim et al., 1993; Gerlach et al., 1994; Faucheux et al., 1995; Loeffler et al., 1995; Ponting, 2001; Bishop et al., 2002), suggesting that this protein may be altered in iron-deficiency. Therefore, this may indicate the involvement of SIRT2 in RLS as iron-deficiency has been commonly observed in RLS patients. Furthermore, as both Parkinson's disease and RLS are both treatable using dopamine agonists (Parkes et al., 1976; Calne et al., 1978; Akpinar, 1982; Earley and Allen, 1996), may further suggest a connection of SIRT2 through both iron and dopamine, particularly as an increase in SIRT2 expression has been suggested in Parkinson's disease (Outeiro et al., 2007).

\subsubsection{Transporter and enzymes}

Investigating candidate markers helped verify our findings, by comparing the identified trends of these markers to those previously described. In the current study, a $10 \%$ decrease in DAT was observed in the midbrain of ID WT mice. Previous ID rodent models also identified a $20 \%$ and $30 \%$ reduction in DAT at the NAcc and caudate putamen regions, respectively (Erikson et al., 2000). A reason for the decrease in DAT expression in iron-deficiency is because DAT requires iron as a coenzyme (Erikson et al., 2000).

The enzyme TH shows an increase in the midbrain of ID WT mice. One would expect a decrease in the expression of this enzyme particularly as $\mathrm{Fe}^{2+}$ is required as a co-factor for TH function during the rate limiting step in the NE synthetic pathway in the conversion of LTyrosine to L-DOPA in presynaptic dopamine neuron terminals (Berger et al., 2002). Previous studies show diversity in the results of this enzyme, for example rodent studies show no change in TH (Connor et al., 2003), while others confirm a 30\% decrease in TH at the caudate putamen (Beard et al., 2007). This difference may be due to the short-lived nature of this 
neurotransmitter enzyme and the quick changing nature of its expression in neural tissue (Berger et al., 1985; Iacovitti et al., 1987).

Finally, ChAT showed a trend to increase slightly in the midbrain of ID WT animals. This may be the result of an increased ACh production or presence of cholinergic terminals within the midbrain region. In addition, this effect may be mediated by neurotrophic factors, where a previous rodent study showed that both nerve growth factor (NGF) and glial cell line-derived neurotrophic factor (GDNF) increased ChAT activity in the striatum and hippocampus (Lapchak et al., 1997). Furthermore, Lapchak and colleagues (1997) showed GDNF to increase TH production within the SN of young rats. Increasing GDNF also elevates dopaminergic neurotransmission (Tomac et al., 1995; Hong et al., 2007) and turnover (Lapchak et al., 1997), which may further explain why TH expression was increased in the current study. Furthermore, a previous 2-D Gel electrophoresis study determined a 38\% decrease in GFAP at the caudate nucleus after 5 weeks of iron-deficiency in the rodent (Youdim et al., 1986). Moreover, GFAP k/o mice are shown to have elevated levels of GDNF in the striatum (Hanbury et al., 2003). This suggests that iron-deficiency in our ID mouse model may reduce GFAP and increase GDNF levels at the midbrain region, subsequently increasing the level of ChAT and TH.

\subsection{Conclusions}

Inducing a nutritional deprivation of iron in mice appears to produce a steady state of irondeficiency with no severe anaemia, as observed by normal haematocrit/haemoglobin levels and only a slight decrease in voluntary wheel-running performance at the beginning of the dark phase. The behavioural studies performed within this thesis describe iron-deficiency in increasing both acute and persistent pain responses through primary A $\delta$ - and C-fibre afferents, which have been shown in clinical studies to be altered in both primary and secondary RLS patients. In addition, the absence of D3 receptors induces hyperalgesia; moreover, a lack of D3 receptors in synergism with iron-deficiency may explain a possible worsening of symptoms, which is also described in the human RLS. The increased c-Fos cellular expression was observed alongside the increased pain sensation in ID WT mice, therefore one may conclude that the increased pain sensation in human RLS may show similar changes in celluar activity at the dorsal spinal cord.

Iron-deficiency was also shown to influence the circadian onset of increased motor activity in WT mice 2 hours before the rest phase (light phase), therefore at a time-point comparable to the onset of symptoms in human RLS. The absence of D3 receptors in 
synergism with iron-deficiency causes a 2-4 hour shift of heightened activity to an earlier time-point, meaning during earlier daytime periods in humans. This shifting effect of increased motor symptoms has been described in human RLS patients under an overdosage of dopaminergic therapy, and is called augmentation. This by definition arises from treatment with dopaminergic agonists and is suggested to be due to a shift of dopmainergic balance from inhibitory D2 towards D1 receptors. It still remains unknown whether drug naive irondeficient RLS patients can show similar effects. In addition, the increase in cholinergic inputs upon motoneurons at the CPG region may give some indication into the increased activity observed in ID WT mice after 15 weeks of diet, however further work is still required.

Certain proteins were dysregulated by iron-deficiency in the midbrain region of male ID WT mice. One protein was partially identified as SIRT2 by MS/MS, however fully identified by PMF. This protein was increased in the midbrain of ID WT mice. Western blotting and 2-D IEF/SDS-PAGE confirmed the increase in SIRT2 expression to arise from the myelin localised short variant (v2). The effect of iron-deficiency in our mouse model resulted in hypomyelination as shown by reduced PLP/DM20 and MOG proteins, also a decrease in DAT expression, confirming previous findings.

These are the first animal studies to describe changes in both sensory and motor behaviour which are comparable to human RLS. Previous animal studies have mainly focused on recreating the motor symptoms of RLS, moreover, to date there are no existing animal studies which have identified sensory changes in ID mice that are comparable to human RLS. Furthermore, this is the first study to characterise SIRT2 as a potential protein involved in iron-deficiency.

\subsection{Current and future research}

Currently, the number of TH positive neurons and their activity at the diencephalic A11 region is being investigated in ID WT and ID $\mathrm{D}^{-/-}$mice. This diencephalospinal dopaminergic region has been suggested in the literature as a structure participating in sensorimotor behaviour associated with RLS (Rye, 2004), although its function has not been clearly explored in a possible animal model. As the voluntary wheel-running, hot-plate and formalin-tests have been successful in evaluating both RLS-like motor and sensory components in mice, they will be used for investigating whether similar changes are also seen in aged ID mice, i.e. at an equivalent age of onset for secondary RLS in humans. In addition, testing pharmaceutical compounds used for treating RLS patients will prove important when validating this animal model. Recent findings from autopsy samples show altered myelin in RLS patients 
(EURLSSG Meeting 2008, Allen et al., unpublished data). In light of this, repeat experiments are currently being performed using 2-D DIGE and MALDI-TOF-MS to re-investigate proteins from myelin fractions as opposed to whole tissue lysate, and to evaluate other proteins at the midbrain and spinal cord which are altered by iron-deficiency. 


\section{Chapter 4}

Materials and Methods 


\section{Materials and Methods}

\subsection{Animals}

Concerning the weight, haematology, sensory behaviour experiments and c-Fos immunohistochemistry, one hundred and nineteen male WT mice were purchased from Harlan Winkelmann GmbH (Borchen, Germany) at the post-natal age of P28. Six groups composed of seventy-seven male WT mice were used for determining weight, plasma-iron, haemoglobin and haematocrit. The groups were $0,1,4,8,15$ and 30 weeks, where for each group 7 control and 7 ID were used. The 0 week group consisted of only 7 control mice. Forty-two male WT mice were used for the initial hot-plate and formalin behavioural studies and c-Fos assessment. Behavioural studies were performed in three different groups, 1, 4 and 15 weeks of diet duration P28, where 14 mice (control and ID) were used per group. After completion of the behavioural pain tests, these mice were sacrificed and used accordingly for c-Fos immunohistochemistry. The second set of sensory behavioural tests made use of $14 \mathrm{D}^{-/-}$mice, consisting of 7 ID and 7 control per group. In addition, a further 14 WT mice were purchased where 7 control and 7 ID mice were used per group. The mice purchased for the second set of pain tests were beforehand used for the main wheel-running experiment as described below.

For the preliminary voluntary wheel-running experiments and ChAT immunohistochemistry, thirty male WT mice were purchased from Harlan Winkelmann $\mathrm{GmbH}$ (Borchen, Germany) at P28. Two groups from the thirty animals were recorded continuously for wheel-running behaviour from 2 weeks P28 through to 15 weeks P28. Afterwards, half the animals from the existing two groups were sacrificed and prepared for 2D gel electrophoresis and MALDI-TOF-MS. The remaining 7 ID and 7 control mice were prepared for ChAT immunohistochemistry forming the 15 week ID diet group. A further 14 WT mice were purchased and grouped, 7 ID and 7 control diet starting P28. They were also placed on the running wheel at a comparable age and stage in diet duration. After completing 2 weeks wheel-running, they were sacrificed forming the 4 week ChAT immunohistochemistry group. For the main wheel-running experiment, a total of $30 \mathrm{WT}$ mice were purchased and grouped (15 ID and 15 control) accordingly. In addition, $30 \mathrm{D}^{-/-}$mice were bred and grouped (15 ID and 15 control), for the same voluntary wheel-running experiment. Afterwards these mice were tested for both hot-plate and formalin-tests.

\subsubsection{Breeding}

Thirty male WT mice were purchased from Charles River Laboratories GmbH (Sulzfeld, Germany) at P28. A total of 3 breeding pairs homozygous deficient for dopamine receptor 3 $\left(\mathrm{D}^{-/}{ }^{-}\right)$from a C57B1/6 background strain, were a kind gift from Professor Christine 
Stadelmann (Department of Neuropathology, University Medical Centre Göttingen, Göttingen). Prior to breeding of D3-/- mice, the genotyping of tail DNA was pre-determined by polymerase chain reaction (PCR). From the breeding pairs a colony of $\mathrm{D} 3^{-/-}$mice was made, where thirty $\mathrm{D}^{-/-}$mice at $\mathrm{P} 28$ were used for wheel-running experiments and pain tests at the same age and in parallel to WT mice.

\subsubsection{Housing conditions}

Mice were maintained in separate cages in the same quiet animal room within the ZTE Göttingen and under the same ambient conditions, 12 hour light/dark cycle (lights on 07:00hr, lights off 19:00hr), humidity (40-55\%) and at a set ambient temperature $\left(23^{\circ} \mathrm{C} \pm 1^{\circ} \mathrm{C}\right)$. All mice were handled and weighed every two days for the first week, thereafter weighing continued once a week. All weights were logged and acted as a reference for the general health of the animal, especially during the first two weeks. Entering the animal house was restricted to the following hours $07: 00 \mathrm{hr}-08: 30 \mathrm{hr}$ in the morning and similarly in the late afternoon/early evening 17:30hr-19:00hr.

Prior to and during every experiment mice were maintained in clear Perspex cages ( $\mathrm{L} x$ $\mathrm{W} \times \mathrm{H}, 26 \mathrm{~cm} \times 18 \mathrm{~cm} \times 14 \mathrm{~cm})$, with animal bedding from LIGNOCEL ${ }^{\circledR}$ (JRS-J.Rettenmaier \& Söhne GmbH + Co. KG, Rosenberg, Germany) enclosed by a stainless steel-barred cage top. Cages were cleaned and new bedding added weekly. Water and food were available ad libitum. All animals used for the research purposes of this thesis, were maintained at the ZTE animal facilities (Göttingen, Germany). All experiments were conducted under the German animal protection laws and protocols approved by the Government of Lower Saxony.

\subsubsection{Diets}

To test the effects of ID diet mice were given a special formulated diet with a reduced ironcontent $(<8 \mathrm{mg} \mathrm{Fe} / \mathrm{kg}$ feed), where control mice were maintained on normal diet (179 mg $\mathrm{Fe} / \mathrm{kg}$ feed). Diets were acquired (ssniff Spezialdiäten GmbH, Soest, Germany) and administered to mice from age P28 and onwards. Both food and water were available ad libitum.

\subsection{Assessing sources for iron-content}

The amount of iron-consumption by the test mice was an important factor when designing the experiments. Before starting the experiments, all components of the cage were checked for iron-content to ensure mice receiving iron-free diet were not obtaining iron from another source, thus invalidating the experiment. This was of great importance, especially when 
investigating iron-deficiency as the main theme for this thesis. Firstly, bedding and water from both tap and automatic dispenser sources were analysed for iron using spectrophotometry (Roche/Hitachi MODULAR ${ }^{\circledR}$ ANALYTICS P 800) at the department of Clinical Chemistry, UMG, Göttingen, Germany. In addition, all other components of the cage including were investigated for iron content. The water used for all animals was from the tap in the animal house, as the concentration of iron $(\mu \mathrm{M} / \mathrm{L})$ was identical to that found in de-ionised or $\mathrm{ddH}_{2} \mathrm{O}$ (see section 2.1).

\subsection{Behavioural experiments}

\subsubsection{Forelimb grip-strength test}

The grip-strength test is already established in mice for testing neuromuscular integrity and muscle strength. A standard trapezoidal grip was attached to the sensor module of a TSE GripStrength-Meter 303500 (TSE Systems GmbH, Bad Homburg, Germany) set to a range of 500. The mouse was allowed to grasp the grip with its forelimbs where it was slowly pulled away by its tail in the same plane to the force meter and horizontal to the base plate. When the mouse released the grip, the maximum force was displayed. This test was performed according to (Souayah et al., 2008). Each mouse was tested in 3 consecutive trials, where the highest score was recorded.

\subsubsection{Hot-Plate test}

The hot-plate test was performed according to (Sanoja and Cervero, 2008) and implemented experimentally to test the acute pain response in both control and ID mice after a period of 1, 4 and 15 weeks of a control or ID diet, starting from P28. The mouse naive to the heat stimulus was placed on to a $50^{\circ} \mathrm{C}\left( \pm 0.5^{\circ} \mathrm{C}\right)$ hot-plate (MEDAX Nagel hotplate, Rendsburg, Germany). At this point a countdown timer set to 30 seconds was activated. This time acted as a safety limit, whereby if no response was observed within this period the mouse was immediately removed. A second stop clock was started simultaneously to the first, where the time in seconds was recorded, starting from when the mouse was first placed upon the hot-plate to the first hind-paw licking or jumping. Once this behaviour was observed, the mouse was immediately removed from the hot-plate and placed back into its home-cage.

All recordings for this test were performed under single-blinded conditions between the hours of 07:00am-14:00pm under monitored ambient (temperature and humidity) conditions. A pre-determined order alternating between control and ID mice (i.e. control, ID, control, etc.) was implemented in a single-blinded manner prior to recordings. This order was 
unknown to the observer. Practice of the experimental procedure was implemented prior to the final recordings, ensuring the observer was experienced in the quantification method.

\subsubsection{Formalin-test}

A modified protocol performed according to (Luvisetto et al., 2006) was used. The formalintest was used to assess the prolonged inflammatory pain response of control and ID mice. Twenty-four hours after completing the hot-plate test, the same mouse groups $(1,4$ and 15 weeks) used for the hot-plate test experiments were subjected to the formalin-test. This 24 hour time period was chosen due to the transient expression of c-Fos, which would be assessed later as a marker of cellular activity. This ensured that any c-Fos expression induced during the hot-plate test would be absent and therefore not interfere with the expression level induced by the formalin-test. Reasons for this are based on a previous study describing c-Fos expression to have a maximum lifetime of around 16 hours (Presley et al., 1990).

The experiment was started by injecting $20 \mu 1$ of $4 \%$ formalin solution subcutaneous (s.c.) into the dorsal surface of the left hindpaw using a $30 \mathrm{G}$ needle (BD Micro-Fine ${ }^{\mathrm{TM}}$, Becton Dickinson, Mannheim, Germany). All mice were naive to this stimulus. After injecting the formalin, the mouse was immediately placed back into the Plexiglas activity chamber. Once the mouse was returned to the Plexiglas monitoring chamber a 5 minute pre-set countdown timer was started, where an additional timer measured the time in seconds spent licking/lifting/biting the injected left hindpaw within a set 5 minute time epoch. The total time spent licking/lifting/biting within that 5 minute time epoch was summed using a start-stop timer. Continuous recordings were made for each mouse from 0-45 minutes, therefore, nine separate 5 minute time epochs were quantified. Immediately after completing the formalintest, the difference in thickness between the formalin injected ipsilateral hindpaw and the noninjected contralateral hindpaw was measured ( $\mathrm{mm}$ ) using a calliper, to produce a value denoted as the inflammation score.

Every two days for one week prior to experiments, all mice were habituated to the room's ambient conditions and Plexiglas chamber for 10 minutes at a time similar to when the experiments would be performed. The mouse was placed into a clear Plexiglas activity chamber $(\mathrm{L} \times \mathrm{W} \times \mathrm{H}, 33 \mathrm{~cm} \times 33 \mathrm{~cm} \times 39 \mathrm{~cm})$ at a recorded room temperature $\left(22^{\circ} \mathrm{C}\right)$ and humidity $(40 \%)$, on a desktop surface $\left(21^{\circ} \mathrm{C}\right) 7$ minutes before starting the experiment.

Similarly to the hot-plate experiments, the observer was experienced in the injection and quantification methods prior to performing the final experiments. Furthermore, a similar pre- 
determined order to test the animals was made as described above for the hot-plate test. This order was single-blinded, therefore unknown to the observer.

\subsubsection{Voluntary wheel-running experiments}

After grouping and for the initial wheel-running experiment, there were 15 test $(n=15)$ and 15 control $(n=15)$ mice, where another $n=15$ per group were used for the repeat experiment. After 2 weeks, a running wheel was added with a non-iron metal bar to help separate the bedding from the wheel preventing the running wheel from becoming blocked. A running wheel was kept in each animal's cage until the end of the experiment. A 1 week training period was implemented based on previous findings in laboratory mice, which show increased running activity until a plateau phase is reached after approximately 5 days (Festing and Greenwood, 1976; Sherwin, 1998). During this 1 week training period animals became familiar to the running wheel and increased their running performance due to musculo-skeletal and cardiopulmonary training. If animals failed to run abnormally during the 1 week training period they were automatically excluded prior to the start of the experiment. Also, only those mice which showed a dark phase running performance above $10 \%$ of the group average were included in the study. The experiment started when the training period finished, where at this point mice were aged 7 weeks and had been on control or ID diets for 3 weeks. Data was collected for the 4 and 15 week diet durations, where the raw data from both diet duration periods consisted of 6.75 consecutive days. In some instances, more control and test mice stopped performing when the 15 week diet duration was reached. These mice were therefore excluded from the 15 week dataset. The number of ID/control WT mice used for 4 and 15 weeks were, $n=15 / n=12$ and $n=14 / n=11$, respectively. In addition the number of ID/control D3 $3^{-/-}$mice used for 4 and 15 weeks were, $n=15 / n=13$ and $n=14 / n=13$, respectively.

Additionally, during the training period the recording devices were activated and left active throughout the duration of the experiment. The axes of the running wheels were coated with Teflon to reduce noise made by contact between the wheel and the holder, therefore minimising noise inside the animal room.

\subsection{Programming for voluntary wheel-running experiments}

All mice had continuous access to a running wheel placed inside their cages during the entire duration of the experiment, which lasted 0-15 weeks P28 of ID diet. The axis of each running wheel was connected to a rotation sensor with a resolution of 16 counts/revolution, where each revolution corresponds to $35.5 \mathrm{~cm}$. Using a customised recording device and software (Boenig und Kallenbach oHG, Dortmund, Germany), wheel-running revolutions were recorded 
continuously at a sampling rate of $1 / 0.48$ s. From this data the following parameters were programmed; accumulative distance in metres (Distance), the maximum velocity $\left(\mathrm{V}_{\max }\right)$, the distance per run $\left(\right.$ Distance $\left./ \mathrm{N}_{\text {run }}\right)$, the number of runs $\left(\mathrm{N}_{\text {run }}\right)$, the total activity time $\left(\mathrm{T}_{\text {run }}\right)$, Percentage of max $\mathrm{N}_{\text {run }}(\%)$ and Percentage of $\max \mathrm{T}_{\text {run }}(\%)$. The last two parameters correspond to the proportion number of runs or activity time observed at every hour in relation to the maximum number of runs or activity time achieved by each individual animal between the hours 19:00hr-03:00hr. All parameters were calculated for time intervals of one hour using a custom designed MatLab program (The MathWorks, Inc., Natick, MA, USA). Data was binned by the program so that 30 minutes was included prior to and after every time-point. Therefore, data represented in the results section shows the 12 hour dark phase as 19:00hr07:00hr, meaning that at the time-points $19: 00 \mathrm{hr}$ and $07: 00 \mathrm{hr}, 30$ minutes of each time-point lies within the light phase.

We chose a continuous access to the running wheel to avoid any influence of exploring behaviour which would occur if the wheels were only temporarily placed inside the cages.

Graphical visualisations were developed using Microsoft Excel. Particular time epochs were created by programming, where the corresponding data to these epochs were later analysed in Microsoft Excel. A time epoch was made every one hour to observe the general circadian activity and every two hours to amplify a certain effect.

\subsection{Anaesthesia and blood extraction}

\subsubsection{Anaesthesia}

Mice were brought 30 minutes into the surgery room prior to being anaesthetised with 14/40 units of Rompun ${ }^{\circledR} 2 \%$ (Xylazinehydrochloride) (Bayer Vital GmbH, Leverkusen, Germany) and the remaining 26/40 units with Ketamine 100 (Ketaminehydrochloride) (Pharmanovo $\mathrm{GmbH}$, Hannover, Germany). This solution was injected through an intra-peritoneal (i.p.) route producing a humane overdose.

\subsubsection{Extracting blood from the mouse}

Once a certain level of anaesthesia was reached, which was tested by the hindpaw pinch reflex test using rat-toothed forceps, Softasept ${ }^{\circledR}$ N 100\% ethanol spray was sprayed onto the skin prior to incision. Using a sharp scissor the skin and peritoneum were perforated at the mid-line region of the abdomen. The cut was continued to the upper thorax region where the heart was located and exposed. Withdrawing the blood involved using a $1 \mathrm{ml}$ Omnifix ${ }^{\circledR}$ U-40 Insulin $1 \mathrm{ml} / 40$ I.U. syringe (B. Braun Melsungen AG, Melsungen, Germany) attached to a BD Microlance $^{\mathrm{TM}}$ 3. $22 \mathrm{G}$ x 1 ${ }^{1 / 4}$, $-\mathrm{Nr}$ 12. 0.7 x 30mm needle (Becton Dickinson GmbH, Mannheim, 
Germany). The needle was inserted $2 \mathrm{~mm}$ into the right ventricle, followed by slow withdrawal of approximately $0.75 \mathrm{ml}$ venous blood in accordance with the heart beat. This avoided sudden collapse of the right ventricle. Next $70 \mu \mathrm{l}$ of blood was inserted into a potassiumethylenediaminetetraacetic acid (EDTA) coated blood vial (SARSTEDT, Nümbrecht, Germany) and immediately shaken to allow mixture of EDTA and blood preventing coagulation. The remaining blood was placed into a normal $1.5 \mathrm{ml}$ non-EDTA/heparin coated eppendorf.

\subsubsection{Preparing blood for analysis}

Firstly the blood contained within the non-EDTA/heparin coated blood vial (SARSTEDT, Nümbrecht, Germany) was allowed to incubate at room temperature for 1-2 hours, before centrifuging at $13.8 \times 1000 \mathrm{rpm}$ at $20^{\circ} \mathrm{C}$ for 10 minutes using an eppendorf $5415 \mathrm{R}$ centrifuge.

\subsubsection{Diluting plasma samples prior to analysis}

A mouse does not have large quantities of blood available like humans, making multiple analyses more restrictive. Removing blood from the heart is the only way to ensure maximal blood collection, however the blood volume was still in the range of $200-800 \mu$. The amount of plasma obtained (range 50-350 $\mu$ ) after centrifuging the blood samples was considerably less compared to the original blood volume. The available spectrophotometric analyser at the Department of Clinical Chemistry, UMG, would only analyse a minimal sample volume of $200 \mu 1$, or optimal analysis with a volume within the range of $300 \mu 1$. Many mouse plasma samples were below the minimum volume required, therefore, beforehand an initial serial dilution was made to determine the optimal dilution and volume. Obviously, a low dilution volume proved better, however it was important to identify the ideal dilution factor with the smallest error, with relation to the original undiluted plasma sample. Overall a 1:4 dilution proved optimal, and was subsequently used when diluting all plasma samples before iron analysis.

\subsubsection{Plasma-iron}

From observing the levels of iron within the plasma, one can access the level of irondeficiency in the mouse. Ideally serum ferritin is a better representative measure of iron within the brain as the majority of iron presented in the brain is in ferritin form (Earley et al., 2000; Mizuno et al., 2005a). However, due to the larger volume requirement serum could not be used and at the time a working kit for mouse ferritin was not available, therefore it was decided to continue with measuring plasma-iron. 
The pre-aliquoted $70 \mu \mathrm{l}$ plasma samples were pre-stored at $-20^{\circ} \mathrm{C}$. Prior to analysis by spectrophotometry, plasma eppendorfs were dethawed at room temperature and then centrifuged down at $13.4 \times 1000 \mathrm{rpm}$ at $20^{\circ} \mathrm{C}$ for 5 minutes (eppendorf $5415 \mathrm{R}$ centrifuge) to remove debris after freezing. Afterwards, the clear plasma supernatant was aspirated off and placed into a new eppendorf tube, where $210 \mu 1$ of de-ionised water was added and vortexed gently, producing a 1:4 dilution. The amount of $280 \mu 1$ was the advised amount in order to produce a good iron-concentration reading by spectrophotometric analysis. In addition to the 1:4 diluted plasma samples, an internal control of the diluent was required. Therefore, $280 \mu 1$ of de-ionised water, from the same source used to make the dilutions was pipetted into 2 seperate eppendorfs. For every batch of new samples a similar dilution and internal control of diluent was prepared. A Roche/Hitachi MODULAR ${ }^{\circledR}$ ANALYTICS P 800 (Department of Clinical Chemistry, UMG, Göttingen, Germany) was used to determine plasma-iron levels.

\subsubsection{Haematocrit}

Part of the $70 \mu \mathrm{l}$ blood within the potassium-EDTA coated blood vial (SARSTEDT, Nümbrecht, Germany) was used to fill 2/3 (approximately 10 $\mu$ l) of two non-heparin coated haematocrit capillaries (Hirschmann ${ }^{\circledR}$ Laborgeräte). Two capillaries were filled, allowing a double determination to be made ensuring an accurate haematocrit level (\%). Samples were centrifuged for 3.5 minutes at 4000rpm using a Haematocrit (Hettich) centrifuge. Capillaries were removed carefully, preventing disturbance of the plasma/red blood cell (RBC) boundary. Next the percentage measure chart was used, where the capillary was moved until the top of the plasma level was at $100 \%$ and the bottom RBC sediment was at $0 \%$. The percentage (\%) value of the plasma/RBC boundary was then recorded, where the percentage value of RBCs represented the packed cell volume (PCV).

\subsubsection{Haemoglobin}

The remaining blood contained in the EDTA-coated tube was used to measure the haemoglobin level. Firstly, 5ml Haemoglobin FS solution (DiaSys) (Rolf Greiner BioChemica, Flacht, Germany) was pipetted into a plastic test tube using a Dispensette ${ }^{\circledR}$ (Brand). A Blaubrand ${ }^{\circledR}$ intraMARK glass capillary was used to uptake $20 \mu \mathrm{l}$ blood which was then added into the $5 \mathrm{ml}$ Haemoglobin FS solution. A double determination was required; therefore this was repeated twice resulting in two tubes each containing $20 \mu 1$ blood and $5 \mathrm{ml}$ Haemoglobin FS solution. The blood and Haemoglobin FS solution were mixed in the glass test tube by gently inverting. Afterwards, all samples were left for 4 minutes before analysis could start. A $2 \mathrm{ml}$ volume of Haemoglobin FS solution was pipetted into a glass cuvette, this acted as the blank for calibrating the haem-photometric reader. After the blank sample was 
measured, half of the test sample volume was pipetted into a separate glass cuvette and briefly measured at $\lambda=540 \mathrm{~nm}$ using an Eppendorf ECOM 6122 haem reader, this acted as the probe. Afterwards, the remaining half of the sample was placed into another cuvette and measured at $\lambda=546 \mathrm{~nm}$, where the final haemoglobin concentration was then recorded as grams per deciliter $(\mathrm{g} / \mathrm{dL})$.

\subsection{Tissue extraction and immunohistochemistry}

\subsubsection{Tissue preparation, sectioning and storage}

After extracting blood from the right ventricle, transcardial perfusion was performed through the left ventricle with $40 \mathrm{ml}$ Isotone Kochsalz- Lösung 0.9\% sodium chloride $(\mathrm{NaCl})$ Braun $(\mathrm{B}$. Braun Melsungen AG, Melsungen, Germany) exsanguinating intravenous blood. Afterwards $4 \%(\mathrm{w} / \mathrm{v})$ paraformaldehyde (PFA) $+100 \mathrm{mM}$ phosphate-buffered saline (PBS) was transcardially perfused through the same left ventricle catheter $21 \mathrm{G}^{3} / 4$ VACUTAINER $^{\circledR}$ (Becton Dickinson GmbH, Mannheim, Germany).

Afterwards, the spinal cord was removed by cutting away the vertebral column. Once the ventral side of the complete spinal cord was exposed, the spinal nerves exiting the vertebrae through the intervertebral foramen were examined under an Olympus SZ61 dissecting microscope (x14.5 combined magnification), with an Olympus KL 1500 LCD light source. The T13-L2 and L4-L6 regions were identified and were dissected using a GEM ${ }^{\circledR}$ stainless steel razor blade (PLANO GmbH, Wetzlar, Germany). The cut spinal cord regions were gently lifted out using a blunt-ended tweezer and placed into a separately labelled (T13L2 or L4-L6) $15 \mathrm{ml}$ falcon tube containing $4 \%$ PFA $+100 \mathrm{mM}$ PBS for $2-4$ hours at $4{ }^{\circ} \mathrm{C}$. The T13-L2 and L4-L6 spinal regions were used for ChAT-IR labelling of ventral motoneurons and c-Fos/c-Jun immunolabelling of dorsal horn cells, respectively. In addition, the diencephalic A11 region was also examined and prepared in accordance to the Paxinos Mouse Brain Atlas (Bregma -1.94mm, Dorsal 1.5mm) (Paxinos, 2003) and forms part of an ongoing study.

After post-fixation, the 4\% PFA was decanted off the spinal cord tissue, which were then briefly washed with $100 \mathrm{mM}$ PBS. Next $30 \%$ (w/v) sucrose $+100 \mathrm{mM}$ PBS was added to the tissue and left for 24 hours at $4^{\circ} \mathrm{C}$. Afterwards, the tissue was then removed and three T13L2 spinal cord regions were placed in the correct orientation upon the chuck and frozen embedded using Sakura ${ }^{\circledR}$ Tissue-Tek $^{\circledR}$ O.C.T. ${ }^{\mathrm{TM}}$ (Heppenheim, Germany) compound at $-20^{\circ}$ C. Cryosections of $10 \mu \mathrm{m}$ and $30 \mu \mathrm{m}$ were cut using a Leica CM3050 S cryostat (Leica Microsystems $\mathrm{GmbH}$, Wetzlar, Germany) and placed onto glass slides (Menzel-Glaser ${ }^{\mathbb{R}}$ SuperFrost ${ }^{\circledR}$ Plus slides $(25 \times 75 \times 1.0 \mathrm{~mm})$ (Menzel GmbH \& Co KG, Braunschweig, Germany) 
at room temperature $\left(20-25^{\circ} \mathrm{C}\right)$. Afterwards sections were allowed to dry for 1-2 hours at room temperature prior to placing into a slide box and storing at $-20^{\circ} \mathrm{C}$. A total of approximately 14 spinal cord sections per animal were kept for analysis. One additional slide was used for the negative control of the antibody. The quality of the sections was determined by examining under light level x10 and x20 magnification using an Olympus BX41 light microscope (Olympus, Hamburg, Germany).

\subsubsection{Immunohistochemistry for c-fos at L4-L6}

The level of c-Fos expression was investigated after completion of the formalin-test to determine whether different durations of ID diet (1, 4 and 15 weeks), affected the level of formalin induced central cellular activity.

A 50 minute period after the formalin injection was chosen as the number would be representative of the final stages of the formalin-test and in addition represents a time-period whereby c-Fos expression is mainly found in the ipsilateral horn region (Ji and Rupp, 1997). In addition, around 60 minutes has been shown to be the optimal time-point for maximal c-Fos expression at superficial laminae I/II at L4-L6 region (Presley et al., 1990).

Directly after the formalin injection, each mouse was moved to a separate room for the anaesthesia followed by transcardial perfusion using 4\% PFA. The spinal cord was removed and the L4-L6 region was identified and separated. The L4-L6 spinal tissue was post-fixated in $4 \%$ PFA for 3 hours at $4^{\circ} \mathrm{C}$. Afterwards, the tissue was cryoprotected in $30 \%$ sucrose + $100 \mathrm{mM}$ PBS for 24 hours at $4^{\circ} \mathrm{C}$ prior to cryosectioning at $10 \mu \mathrm{m}$ thickness.

\subsubsection{Immunoperoxidase method}

A hydrogen peroxide solution was used to block endogenous enzyme activity before immunostaining the tissue sections using a standard peroxidise method. A $10 \%$ foetal calf serum (FCS) + 1M PBS block solution was applied prior to using a 1:1000 in 10\% FCS + 1M PBS rabbit anti-c-Fos polyclonal IgG (Santa Cruz Biotechnology, Heidelberg, Germany) primary antibody for 24 hours at $4^{\circ} \mathrm{C}$. The secondary biotinylated anti-rabbit antibody (GE Healthcare Europe, Freiburg, Germany) was diluted 1:200 in 10\% FCS + 1M PBS and incubated for 1 hour at room temperature followed by a 1 hour incubation at room temperature using 1:1000 + 1M PBS POX ExtrAvidin ${ }^{\circledR}$-Peroxidase (Sigma-Aldrich, Steinheim, Germany). A 2\% 3,3'-Diaminobenzidine tetra-hydrochloride (DAB) (Sigma-Aldrich, Steinheim, Germany) solution was applied to the spinal sections for 3 minutes. Sections were subjected to dehydration followed by mounting in DePeX (Serva, Heidelberg, Germany) and then coverslipped. Slides were stored at room temperature. 
A negative control was used beforehand to determine whether non-specific binding of the secondary antibody was occurring. This process involved the same immunohistochemical technique except no primary antibody was added, instead 10\% FCS + 1M PBS was used.

\subsubsection{Immunofluorescence method}

Fluorescent staining of c-Fos involved the same procedure and dilutions as described above for the peroxidase method, with the exception of using a different secondary antibody. The indocarbocyanine 3 (Cy3) conjugated Donkey Anti-Rabbit IgG $(\mathrm{H}+\mathrm{L})$ secondary antibody (DIANOVA GmbH, Hamburg, Germany) was diluted 1:300 in 10\% FCS + 1M PBS and applied for 2 hours at room temperature. Afterwards, a wash cycle was made and then the nuclear marker 4',6-diamidino-2-phenylindole (DAPI) (Invitrogen, Karlsruhe, Germany) at a concentration of 1:10000 in 1M PBS was applied to the sections for 15 minutes, where afterwards a further wash cycle step was made. DAPI is a strong toxic and mutagenic compound which binds extensively to DNA, therefore allowing nuclei to be visualised. The choice of another fluorophore in addition to labelling with DAPI, meant the second fluorophore must possess a different emission wavelength $(\lambda)$ to DAPI, which has an emission maximum of $\lambda=461 \mathrm{~nm}$ observed at the blue/cyan end of the visible spectra. The second fluorophore of choice was Cy3 which has an emission maximum of $\lambda=570 \mathrm{~nm}$, observed at the red end of the visible spectra. This combination of DAPI and Cy3 allows both signals to be determined, therefore making double labelling possible.

A negative control for the fluorescent stained sections was used to determine whether non-specific binding of the secondary antibody was occurring. This process involved the same immunohistochemical technique, however the only difference was that $10 \%$ FCS $+1 \mathrm{M}$ PBS was applied instead of the primary antibody.

After washing the sections, they were then mounted in Vectashield (AXXORA GmbH, Lörrach, Germany) and coverslipped using a Menzel-Glaser ${ }^{\circledR}(24 \times 24 \mathrm{~mm})$ (Menzel GmbH \& Co KG, Braunschweig, Germany) coverslip. Clear nail vanish was applied around the edge of the coverslip preventing the mounting medium from leaking away from the tissue sections.

All biotinylated stained slides were maintained at room temperature, fluorescent stained sections at $4{ }^{\circ} \mathrm{C}$ and the unstained sections were kept at $-20^{\circ} \mathrm{C}$.

\subsubsection{Immunohistochemistry for ChAT at T13-L2}

Sections of $30 \mu \mathrm{m}$ thickness were removed from $-20^{\circ} \mathrm{C}$ storage and allowed to stand for 60 minutes at room temperature. Using a wax mini-PAP pen (Invitrogen, Karlsruhe, Germany) a circle was made around the sections, to help retain the reagents upon the spinal sections. 
Afterwards, 100mM PBS was applied to the sections for 5 minutes to rehydrate the tissue. Prevention of non-specific binding requires a "blocker" to be used, thus a blocking solution containing 10\% (v/v) Normal Donkey Serum (NDS) (DIANOVA GmbH, Hamburg, Germany) + 0.3\% (v/v) Triton X-100 (ICN Biomedicals GmbH, Meckenheim, Germany) + $100 \mathrm{mM}$ PBS was applied to the T13-L2 spinal cord sections for a period of 30 minutes at room temperature, afterwards a wash cycle was made. Every wash cycle throughout the immunohistochemical procedure used the same wash cycle, which consisted of 2 x 5 minute washes followed by 2 x 15 minute washes using 100mM PBS. The Goat anti-ChAT polyclonal IgG (Millipore GmbH, Schwalbach, Germany) primary antibody was diluted 1:100 in 1\% NDS $+0.03 \%$ Triton $\mathrm{X}-100$ for 24 hours at $4^{\circ} \mathrm{C}$, where afterwards a wash cycle was made. The Cy3 conjugated Donkey Anti-Goat IgG $(\mathrm{H}+\mathrm{L})$ secondary antibody (DIANOVA GmbH, Hamburg, Germany) was diluted 1:200 in 100mM PBS and applied for 2 hours at room temperature. After washing the sections they were mounted in Vectashield (AXXORA GmbH, Lörrach, Germany) and coverslipped using a Menzel-Glaser ${ }^{\circledR}(24 \times 24 \mathrm{~mm})$ (Menzel GmbH \& Co KG, Braunschweig, Germany) coverslip. Clear nail vanish was applied around the edge of the coverslip, sealing the mounting medium upon the sections.

All stained and unstained sections were kept at $-20^{\circ} \mathrm{C}$. A negative control was used to determine whether non-specific binding of the secondary antibody was occurring.

\subsubsection{Photoimaging and quantification}

Imaging of all c-Fos biotinylated DAB sections were made using the light function on the Olympus BX51 microscope (Olympus, Hamburg, Germany). Images were captured at x10 magnification with a fitted Olympus DP71 camera and saved using Olympus Soft Imaging Solutions (Soft Imaging System, Olympus, Hamburg, Germany) Cell F software. Every image was subjected to the same brightness and contrast settings using Cell F.

The total number of c-Fos-IR nuclei within laminae I and II were counted under singleblinded conditions from both the ipsilateral and corresponding contralateral dorsal horns. These two superficial laminae are known to be important in receiving primary afferents relaying information from, nociceptors, thermoreceptors and mechanoreceptors (Shepherd, 1994). All images were stored and recorded. Thirteen sections from each mouse were selected in a random manner for subsequent quantification.

c-Fos and ChAT fluorescent images were made using an Olympus BX51 fluorescence microscope (Olympus, Hamburg, Germany) with dichroic filters (DAPI, fluorescein isothiocyanate (FITC), and $\mathrm{Cy} 3$ ) fitted into the filter cube turret of the microscope. Images were captured at $\mathrm{x} 10$ and $\mathrm{x} 40$ magnifications. With regards to ChAT immunohistochemistry, a 
fixed plane of focus was made for all images by ensuring the nuclei of the motoneurons had the sharpest point of focus. The manual settings of $30.30 \mathrm{~ms}$ exposure time, a sensitivity of ISO 800 and spot size $0.1 \%$, were used for all images. All images were stored and recorded. Fourteen sections from each mouse were selected in a random manner for subsequent quantification.

The number of ChAT-IR boutons in contact with the outer surface of the motoneuron were counted, were this parameter was only implemented for area 2. In addition, the area of the motoneuron and perimeter were quantified by a draw feature in the Cell F program for all neuron groups in each image and for each of the 2 predefined spinal regions of interest. The areas of interest were, area 1 which is the IML of lamina VII, and area 2 which has two theoretical regions (lateral and medial nuclei of lamina IX and VIII), however only one region was distinguishable in the ventral horn, which has important implications in motor function. 


\subsection{Tissue and protein preparation}

\subsubsection{Stereotaxic positions of the midbrain}

The online Allen brain atlas was used to identify the stereotaxic coordinates relating to the areas of interest, for subsequent dissection and protein analysis.

The distance between the start and end of the midbrain region was required; therefore, three stereotaxic coordinates were needed. The initial cut, point 1 (P1), was made using a $\mathrm{GEM}^{\circledR}$ stainless steel razor blade (PLANO GmbH, Wetzlar, Germany) at bregma $-3.780 \mathrm{~mm}$ (see Fig. 4.1) found at Atlas-Coronal-166 (position: 3630). Rostral from Point 1, the next cut termed point 2 (P2), was made at bregma $-0.755 \mathrm{~mm}$ Atlas-Coronal-283 (Position: 6204). The region between (P2-P1) included the entire midbrain region (see Fig. 4.1). The third and final cut, point 3 (P3) was made at bregma $+2.745 \mathrm{~mm}$ Atlas-Coronal-423 (Position: 9284).

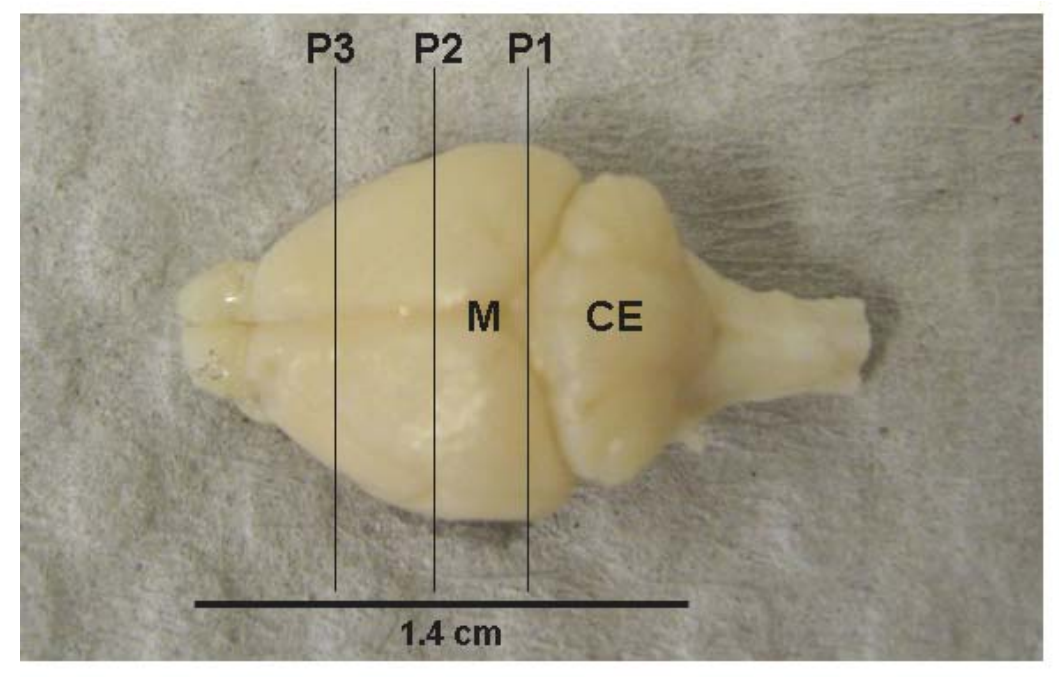

Fig. 4.1. Dissected midbrain region for protein identification. Image of a mouse brain, with lines representating the three points of incision (P1, P2, P3) made using a razor blade. Between P1 and P2 lies the midbrain (M) region. Also the cerebellum (CE) is illustrated here. The coloured stereotaxic images from the Allen brain atlas, shows the rostral perspective of inner structures in relation to the different blade positions.

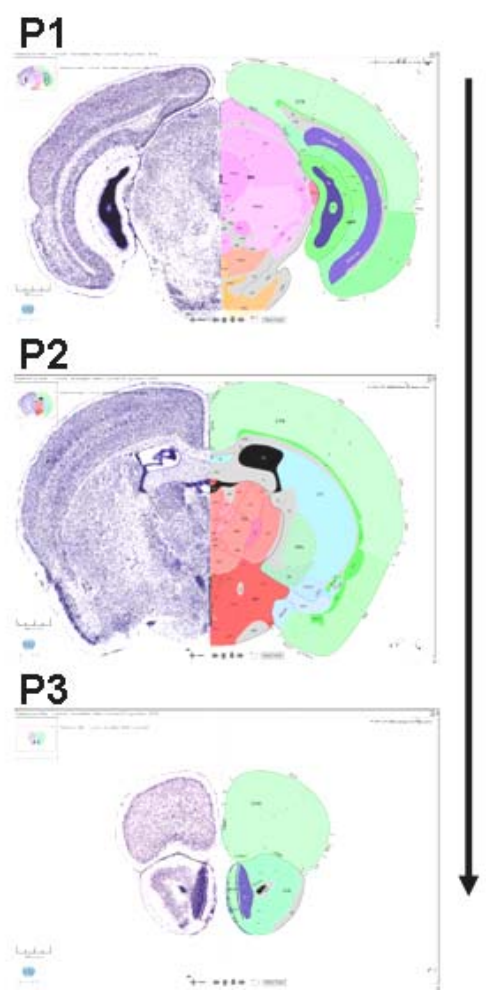

\subsubsection{Tissue preparation}

Animals were killed by cervical dislocation. Afterwards, the mouse was decapitated and the brain removed by cutting away the skull. The initial cut was made at a dorsal position which proceeded rostrally.

Once the brain was removed, it was placed dorsal side-up on a cutting block and cut using a $\mathrm{GEM}^{\circledR}$ stainless steel razor blade (PLANO GmbH, Wetzlar, Germany) at the boundary between the inferior and superior colliculi (P1). The cerebellum was extracted and placed into a $1.8 \mathrm{ml}$ cryotube (Nalgene ${ }^{\circledR}$ Cryoware $^{\mathrm{TM}}$ (Nunc GmbH \& Co. KG, Wiesbaden, Germany). 
Next, a razor blade was inserted into the middle part of a glass cutting block (Braintree Scientific (1 $1 \mathrm{~mm}$ cutting divisions). Afterwards, the brain was placed in close apposition to the razor blade in the block. Next 3 cutting parts (each part is $1 \mathrm{~mm}$ ) were counted, therefore $3 \mathrm{~mm}$ rostral at which point another blade was inserted through the brain, making P2. The final blade position (P3), was inserted $3 \mathrm{~mm}$ rostral to the second razor blade position (P2) (see Fig. 4.1). The tissue section between the first and second blade was the pre-determined midbrain region. Afterwards, the midbrain region was laid flat, where the cortex and insula were removed. The subcortical region, termed midbrain region throughout this chapter, comprised of the striatum, $\mathrm{SN}$, pons and hypothalamus and was placed into an appropriately labelled cryotube. Next, the tissue section between P2 and P3 was separated, where the cortex including corpus callosum was placed into a separately labelled cryotube. Similarly, the entire spinal cord was removed and placed into a separate cryotube. Once the tissue was extracted and placed into a cryotube, it was immediately snap-frozen in liquid nitrogen.

\subsubsection{Tissue homogenisation}

The following were performed at the laboratory of Professor Dr. Med. Markus Otto (Neurology Group, Centre for Clinical Research, Helmholzstraße 8/1, Ulm, Germany). All materials used unless stated otherwise were from GE Healthcare (Freiburg, Germany).

Firstly, lysis buffer was made using 30mM Tris-hydrochloric acid $(\mathrm{HCl}) \mathrm{pH}$ 7.4. + protease inhibitor (Complete Mini, protease inhibitor cocktail tablets, Roche, Mannheim, Germany). Afterwards, tissue samples (midbrain, spinal cord, cortex and cerebellum) from 6 controls (C1-C6) and 6 test mice (ID1-ID6) were homogenised with $0.75 \mathrm{ml}$ of lysis buffer by subjecting to ultrasound sonification, 3 times at 1 pulse every 30 seconds. Homogenate from the sonified tissue was spun down at $13 \times 1000 \mathrm{rpm}$ for 5 minutes at $4^{\circ} \mathrm{C}$. Supernatant was aspirated and stored on ice in an appropriately labelled eppendorf tube. Pellets from the centrifugation were stored at $-80^{\circ} \mathrm{C}$.

\subsubsection{Protein determination of tissue samples}

\subsubsection{Bicinchoninic acid protein colourimetric assay for 2-D Gel electrophoresis}

Quantification of protein levels from each sample (midbrain, spinal cord, cortex and cerebellum) were determined by firstly preparing samples for a (bicinchoninic acid) BCA protein colourimetric assay. This meant having a standard in the assay, which was used to determine the concentration of the protein samples dependent upon their level of absorbance at $\lambda=550 \mathrm{~nm}$. The BCA protein assays were made prior to 2-D DIGE experiments to determine the protein load required for 2-D gel electrophoresis. Previously prepared bovine serum 
albumin (BSA) stocks were dethawed from $-20^{\circ} \mathrm{C}$ to $4^{\circ} \mathrm{C}$ by placing the samples on ice. Next dilutions of these tissue homogenates were required. A 1:2 sample concentration was prepared, from which the following 1:200 and 1:400 dilutions were made in $\mathrm{ddH}_{2} 0$. After making these two concentrations for all 12 tissue samples ( $n=6$ per group), $100 \mu 1$ of each dilution was added to a separate well into a CELLSTAR ${ }^{\circledR} 96$ well assay plate (Greiner BioOne GmbH, Frickenhausen, Germany). In addition, 2 aliquots of $\mathrm{ddH}_{2} \mathrm{O}$ and the following stocks of 5xBSA, 10xBSA, 15xBSA, 25xBSA, 50xBSA, 75xBSA and 100xBSA were added to the assay plate to make an internal control standard. After this was prepared, $100 \mu 1$ of a 1:50 solution was made by adding $100 \mu \mathrm{l}\left(4 \% \mathrm{CuSO}_{4}\right)+4900 \mu \mathrm{l}(\mathrm{BCA})$ into a $15 \mathrm{ml}$ falcon tube, briefly mixing and then pipetting $100 \mu$ of the solution to each well containing a standard or diluted tissue homogenate. Afterwards, parafilm was placed over the plate keeping it dust-free and clean. The plate was then placed into an $80^{\circ} \mathrm{C}$ incubator for 30 minutes. After this period the plate was removed and loaded into a 96 well LP400 enzyme-linked immunosorbent assay (ELISA) microplate reader (Sanofi Diagnostics Pasteur GmbH, Freiburg, Germany). A measurement filter of $\lambda=550 \mathrm{~nm}$ was selected. At $\lambda=562 \mathrm{~nm}$ the copper $/ \mathrm{BCA}$ complex shows a linear absorbance with increasing protein concentration. Between $\lambda=550 \mathrm{~nm}$ and $\lambda=570 \mathrm{~nm}$ the purple colouration can be measured using a colourimetric reader.

The standard was visualised first, where after a scatter plot of $y$-axis concentration $(\mu \mathrm{g} / \mathrm{ml})$ and $\mathrm{x}$-axis absorption was made. A line of best-fit was inserted to produce a standard concentration line. Afterwards $\mathrm{x}$ was substituted for the absorbance value and multiplied by either 200 (for 1:200) or 400 (for 1:400) depending upon which absorbance value was being tested.

Prior to 2-D DIGE experiments, a concentration value was required, i.e. $70 \mu \mathrm{g}$ of tissue was needed for each CyDye: red, yellow and blue. Therefore together a total of $210 \mu \mathrm{g}$ of tissue was needed from each mouse. Firstly, averages of the 1:200 and 1:400 concentration values were made, i.e. (1:200 value $+1: 400$ value/2) then divide again by 1000 as the value was required in $\mu \mathrm{g} / \mu \mathrm{l}$ not $\mu \mathrm{g} / \mathrm{ml}$. The initial reading is in $\mu \mathrm{g} / \mathrm{ml}$ and was divided into the value representing the required protein concentration $(70 \mu \mathrm{g})$, giving the value as $\mathrm{ml}$ of tissue homogenate. However, a $100 \mu \mathrm{g}$ protein load was used for the second 2-D DIGE experiment as illustrated in the results (section 2.6.1.).

The calculated volume in $\mu 1$ for each animal sample was inserted into a new $0.5 \mathrm{ml}$ eppendorf. A volume of 4 times more acetone to the required homogenised tissue volume (usually require 3-10 times of acetone) was added to the probe, producing protein precipitation. All samples were briefly mixed and placed into eppendorfs at $-20^{\circ} \mathrm{C}$. Once the tissue and acetone solution 
mixture is frozen it can be left for long durations, however for these experiments they were left for 48 hours. All remaining protein supernatant was aliquoted into 3 x $0.5 \mathrm{ml}$ eppendorf tubes and placed into a labelled box which was stored at $-80^{\circ} \mathrm{C}$, allowing further analyses of these samples when required.

\subsubsection{2-D Quant protein assay for tank Western blotting}

Quantification of protein levels for each sample (midbrain, spinal cord, cortex and cerebellum) prior to Western blotting, was determined by use of a 2-D Quant Kit. These experiments including Tank Western blotting were performed at the laboratory of Dr Olaf Jahn from the Max Planck Institute for Experimental Medicine (Proteomics Group, Hermann-Rein-Straße 3, 37075 Göttingen, Germany).

A standard curve was prepared from a $2 \mathrm{mg} / \mathrm{ml}$ BSA solution, where the following volumes were added into 6 separate eppendorf tubes: $0 \mu 1,5 \mu 1,10 \mu 1,15 \mu 1,20 \mu 1$, and $25 \mu l$. This produced the following concentrations, $0 \mu \mathrm{g}, 10 \mu \mathrm{g}, 20 \mu \mathrm{g}, 30 \mu \mathrm{g}, 40 \mu \mathrm{g}$, and $50 \mu \mathrm{g}$, respectively. Afterwards, $2 \mu$ from each sample was pipetted into a separate tube. This was repeated twice allowing double determination of each sample. Next $500 \mu \mathrm{l}$ of precipitant from the kit was added to each tube including the standards. A brief vortex was made and then tubes were incubated for 2-3 minutes at room temperature. A further $500 \mu 1$ of co-precipitant was added and the tubes briefly vortexed. Afterwards, all standards and samples were centrifuged at 10 $000 \mathrm{x} \mathrm{g}$ for $5 \mathrm{~min}$. The supernatant was discarded and the tubes were further subjected to another centrifugation step to remove all solution from the pellets. Next, a $100 \mu$ volume of copper solution and $400 \mu \mathrm{l}$ of $\mathrm{ddH}_{2} \mathrm{O}$, were added to each tube and then briefly vortexed to dissolve the precipitant. A volume of $1 \mathrm{ml}$ working colour reagent was added to each tube, where immediately after pipetting this volume the tube was vortexed. All tubes (samples and standards) were allowed to incubate for 15-20 minutes at room temperature. Afterwards, a $200 \mu$ l volume was pipetted off each solution and added onto a CELLSTAR ${ }^{\circledR}$ (Greiner Bio-One GmbH, Frickenhausen, Germany) 96 well assay plate, where the assay was performed using a Bio-Rad Model 680 plate reader set at $\lambda=480 \mathrm{~nm}$. Within 40 minutes after adding the working colour reagent, the samples were assayed. The protein concentration was determined as $\mu \mathrm{g} / \mu \mathrm{l}$, from which the desired protein load could be calculated for Western blotting (see section 4.10). 


\subsection{1-D/2-D gel electrophoresis}

\subsubsection{1-D DIGE gel electrophoresis}

Difference in gel electrophoresis (DIGE) involves modern-day first dimension (1-D) and second dimension (2-D) electrophoresis and is a modified version of two-dimensional polyacrylamide gel electrophoresis. In relation to my project, the two protein samples were labelled separately with Сy3 and indocarbocyanine 5 (Су5), where indocarbocyanine 2 (Сy2) was used in a 50:50 mixture of both protein samples, acting as a standard for the other two dye labelled proteins. Afterwards, the different $\mathrm{Cy} 3$ and $\mathrm{Cy} 5$ images were overlaid with the $\mathrm{Cy} 2$ image, enabling an analysis of difference to be made. Every dye has equal charge therefore not affecting the migration of proteins in the gel, moreover every dye is photostable. In addition, this method allows both test and control samples to be combined on the same gel and minimises the protein load to as little as $125 \mathrm{pg}$.

A total of 6 Immobiline ${ }^{\mathrm{TM}}$ DryStrip pH 3-10 NL of 24cm length, were rehydrated using a rehydration buffer (Urea 7M + CHAPS 4\% w/v $+2 \mathrm{M}$ thioylurea). This was prepared by adding just over $1 \mathrm{ml}$ rehydration buffer to each strip lane in the holding box. Afterwards, the $24 \mathrm{~cm}$ pH 3-10 NL strips were placed into the holding box and left at room temperature. The samples containing tissue acetone solution were taken out of the $-20^{\circ} \mathrm{C}$ freezer and dethawed. Once dethawed, samples were centrifuged at $13 \times 1000 \mathrm{rpm}$ for 5 minutes at $20^{\circ} \mathrm{C}$. This supernatant was discarded and the pellet resuspended in rehydration buffer which contained 30mM Tris$\mathrm{HCl} \mathrm{pH}$ 8.1. Samples were placed into an ultrasound chamber for 1-2 minutes, followed by a short vortex. Afterwards, samples were left overnight on an eppendorf Thermomixer at $1400 \mathrm{rpm}$ at $20^{\circ} \mathrm{C}$, allowing solubilisation to occur. Next, samples were briefly centrifuged at $7 \mathrm{x} 1000 \mathrm{rpm}$ for 20 seconds at $20^{\circ} \mathrm{C}$, where afterwards samples were placed back on ice. A volume of $10 \mu \mathrm{l}$ was pipetted off from each of the 12 samples (6 ID and $6 \mathrm{C}$ ) and placed into one $1.5 \mathrm{ml}$ eppendorf tube that was labelled standard. Next, $3 \times 1.5 \mathrm{ml}$ eppendorf tubes were labelled Cy2, Cy3 and Cy5, where the CyDyes (Cy2 (Yellow), Cy3 (Red) and Cy5 (Blue)) were diluted accordingly with N,N-Dimethylformamide (DMF) from Fluka Biochemika (Sigma-Aldrich GmbH, Taufkirchen, Germany). A dilution of $0.3 \mathrm{nM}$ of dye/gel was required, however, for each run 6 gels were needed ( 1 control and 1 test sample/gel). A protein load of $70 \mu \mathrm{g}$ was used for the initial DIGE experiment 1, where for DIGE experiment 2 a $100 \mu \mathrm{g}$ protein was loaded, resulting in clearer spots on the gels. Before pipetting the CyDyes, the lights were switched off. The dilution was made by pipetting $1.8 \mu$ l of CyDye into appropriately labelled eppendorfs, e.g. a volume of $1.8 \mu \mathrm{l} \mathrm{Cy} 2$ was pipetted into an eppendorf labelled Cy2, where after $4.3 \mu \mathrm{l}$ of DMF was added. Once the 3 eppendorfs were prepared, 
each with a total of around $6 \mu 1$, they were rearranged creating a "Dye Switch." For example, the top row was Cy3 labelled: ID1(Cy3), C2(Cy3), ID3(Cy3), C4(Cy3), ID5(Cy3), C6(Cy3), where the bottom row was Cy5 labelled: C1(Cy5), ID2(Cy5), C3(Cy5), ID4(Cy5), C5(Cy5), ID6(Cy5). A volume of $6 \mu \mathrm{l} \mathrm{Cy} 2$ label was added into the tube containing the standard label, which contains a volume from each sample. When finished all samples containing CyDye were placed on ice for 30 minutes at room temperature and covered with foil to prevent photobleaching. Next, a $10 \mathrm{mM}$ lysine (made in $\mathrm{ddH}_{2} 0$ ) stock was dethawed. The function of lysine is to bind to any unbound label. From this lysine stock $1 \mu \mathrm{l}$ was added to each tube and then $6 \mu l$ lysine to the standard. Directly after pipetting lysine into the eppendorf, it was mixed gently and then left for 10 minutes on ice (covered). Lysis buffer without Tris- $\mathrm{HCl}$ was taken out from $-20^{\circ} \mathrm{C}$ and allowed to dethaw. Next $14 \mathrm{mg}$ DTT was added to $1 \mathrm{ml}$ lysis buffer (without Tris- $\mathrm{HCl}$ ) making a 4\% DTT solution. Afterwards, $40 \mu 1$ of IPG Buffer $\mathrm{pH} 3-10 \mathrm{NL}$ was added to the $4 \%$ DTT + lysis making a $4 \%$ IPG + DTT + lysis buffer solution. Next, the entire Cy5 volume containing the CyDye Switched samples was added to its corresponding Cy3 tube, where only 6 eppendorfs marked ID1(Cy3), C2(Cy3), ID3(Cy3), C4(Cy3), ID5(Cy3), C6(Cy3) remained. Subsequently, 22 $\mu$ l of standard with Cy2 label was added into each of the 6 tubes and mixed using the pipette tip. Afterwards, $22 \mu$ l of IPG/DTT/lysis (without Tris-HCl) buffer was pipetted into each of the 6 tubes and gently mixed. All samples remained on ice until ready for loading into cups for 1-D Gel electrophoresis.

PlusStrip Cover Oil (require 100ml) was poured on top of the 1-D strip holding area. The gel strips were removed from the rehydration buffer using tweezers, where the gel-strip barcode number was recorded. This ensured that the samples contained within each strip were known and under which CyDye Switch conditions. Barcode number 90146-90151 represented samples 1-6 (ID1(Cy3), C2(Cy3), ID3(Cy3), C4(Cy3), ID5(Cy3), C6(Cy3). Gently the gel strip was placed gel-side up with the barcode at the far-end (+ve) of the strip holding region of the 1-D gel apparatus, where the bottom part was at the near end (-ve) of the gel holder. Paper wicks were dampened with de-ionised $\mathrm{H}_{2} \mathrm{O}$ and placed at both ends so that half of the wick overlapped the ends of the strip. Next the electrodes were snapped into position, ensuring the contact lies upon the dampened paper wick. The sample loading cups were also snapped onto the + ve end of the strip. Next the samples were loaded into the cup so that it was below the oil and just above the gel strip. A cover was placed over the 1-D gel apparatus to prevent photobleaching of the CyDyes. The program on the IPGphor ${ }^{\mathrm{TM}}$ system required for isolectric focussing (IEF) of the sample, was set to the following voltage steps: step 1) $100 \mathrm{~V}$ for 30 minutes; step 2) 300Vfor 8 hours; step 3) 1000V for 6 hours; step 4) 8000V for 4 hours; and finally step 5) $8000 \mathrm{~V}$ for 3 hours. The total run-time was approximately 21.5 hours at $4900 \mathrm{~V}$ and $36 \mathrm{KVh}$. At step 1) a $50 \mu \mathrm{A}$ maximum limit was imposed preventing damage to the strips. 
In brief, each step represents the following technical processes; step 1) the samples are entering the gel. At both steps 1) and 2) rough isoelectric focussing occurs i.e. into acid and base. At the higher voltages steps 3), 4) and 5) a finer focussing takes place, whereby proteins are separated with similar $\mathrm{pH}$ values. Eventually every protein reaches a point where no net electric charge is present upon the protein; this is termed the pI value.

\subsubsection{2-D gel polymerisation}

The 2-D gel casting unit was prepared by cleaning the gel casters using $70 \%$ ethanol. The caster was set in an interchanging order of plastic spacer blade and glass gel caster units. The remaining space was filled with plastic sheets making sure that it was not too loose and not too tight, avoiding incorrect gel formation. The outside cover of the gel casting unit was tightened gently, to avoid gels suddenly expanding when removing the caster unit.

A 12.5\% gel contained 30\% Acrylamide/Bis Solution (208.5ml) (Bio-Rad Laboratories GmbH, München, Germany) Tris- $\mathrm{HCl}$ (125ml), ddH $\mathrm{d}_{2} \mathrm{O}(159 \mathrm{ml})$, sodium dodecyl sulfate (SDS) solution (11ml) (Sigma-Aldrich GmbH, Taufkirchen, Germany), 10\% ammonium persulfate (APS) (2.5ml), $N, N, N^{\prime}, N^{\prime}$ tetramethylethylenediamine (TEMED) $(165 \mu \mathrm{l})$ making a $500 \mathrm{ml}$ total. Pouring of the gel solution was performed in a fume cupboard. Next $165 \mu$ of TEMED was added to the gel solution and mixed on a magnetic stirrer. TEMED is used with APS, where APS catalyses the polymerisation process of acrylamide to polyacrylamide. Next the gel solution was poured slowly into the caster unit side-opening. The final gel thickness was $1 \mathrm{~mm}$.

A $3 \mathrm{ml}$ volume of 1-Butanol was pipetted slowly over the top of each gel contained within a glass gel-casting unit. This sealed the gel and prevented dehydration. Finally parafilm was placed over the top part of the gel casting unit which was left in the fume cupboard overnight. The polymerisation time of the gels was approximately 20-24 hours.

\subsubsection{2-D DIGE gel electrophoresis}

2-D electrophoresis of the previously IEF samples required SDS-Polyacrylamide gel electrophoresis (SDS-PAGE), using an Ettan DALTtwelve system separation unit. Firstly, the equilibrium buffer was made using 7M Urea, Tris- $\mathrm{HCl}$ and Glycerol. Afterwards, $36 \mathrm{~g}$ Urea + $3 \mathrm{~g} \mathrm{SDS}+30 \mathrm{~g}$ Glycerol $+3.3 \mathrm{ml}(50 \mathrm{mM})$ Tris- $\mathrm{HCl}$ was added together with $100 \mathrm{ml}$ of $\mathrm{ddH}_{2} 0$ and heated to increase the rate of dissolution. The running buffer Tris-Glycin was prepared. Firstly, 60.5g Tris $+287.7 \mathrm{~g}$ Glycine $+20 \mathrm{~g}$ SDS was made up to a 2 litre volume using $\mathrm{ddH}_{2} 0$. The SDS is $(-\mathrm{ve})$ charged therefore neutralises all charges of proteins. From the equilibration buffer two different solutions were made. Firstly, a reducing agent was made by adding $500 \mathrm{mg}$ DTT to $50 \mathrm{ml}$ of the $100 \mathrm{ml}$ equilibration buffer, producing a $1 \%$ DTT solution. 
Secondly, an alkylation solution was made by adding $2 \mathrm{~g}$ of Iodoacetamide (IAA) to the remaining $50 \mathrm{ml}$ equilibration buffer, producing a $4 \%$ IAA solution.

The Immobiline ${ }^{\mathrm{TM}}$ DryStrips were removed from the 1-D Gel apparatus and placed plastic side-down into a glass cylinder. The $1 \%$ DTT equilibration buffer was poured into the glass cylinder and over the strips and left on a shaking incubator for 20 minutes. This step linearises the protein by breaking disulphide bonds (reducing). After 20 minutes the solution was decanted away from the strips and then replaced by $4 \%$ IAA, where the cylinder was then returned onto the shaking incubator and left for a further 20 minutes. The IAA solution alkylates the proteins preventing S-S bond reformation and preventing protein folding, thus keeping all proteins linearised prior to 2-D Gel electrophoresis.

A $100 \mathrm{ml}$ volume of the 10-fold concentrate running buffer was added to $400 \mathrm{ml}$ of $\mathrm{ddH}_{2}$. This 1:5 solution was poured over the gels in the casting unit to remove excess butanol. Next $750 \mathrm{ml}$ of the 10 -fold concentrate running buffer was poured into the 2-D electrophoresis chamber where 6 litres of $\mathrm{ddH}_{2} \mathrm{O}$ was added.

The Gel caster unit was opened and the glass gel caster plates containing the $1 \mathrm{~mm}$ thick polymerised gels were removed. The outside and top part of the glass gel plates were briefly washed using de-ionised $\mathrm{H}_{2} \mathrm{O}$, removing all traces of butanol.

From the 10-fold concentrate running buffer, $500 \mathrm{ml}$ was diluted with 2 litres $\mathrm{ddH}_{2} \mathrm{O}$ making a 2-fold concentrate of 2.5 litre running buffer. Next the $4 \%$ IAA solution was decanted off the strips. Using a flat-tipped tweezer the strips were removed and then briefly washed in 2-fold running buffer. The strip was then gently pushed until in contact with the top edge of the gel. A $0.5 \%$ agarose +2 -fold running buffer + bromophenol blue solution was heated, where a $1 \mathrm{ml}$ volume of this agarose solution was pipetted above the strip. The solidified agarose secured the position of the strip against the gel, where the addition of Bromophenol Blue acted as a marker to see how fast the gels were running. Afterwards, the glass plates were inserted into the chamber.

The Ettan DALTtwelve 2-D Gel electrophoresis system was then programmed accordingly: step 1) 0.25 Watts/gel for 1 hour; step 2) 0.5 Watts/gel for 1 hour; and finally step 3) $3.5 \mathrm{Watts} /$ gel for 21 hours.

After completion of the 2-D Gel electrophoresis step, the plates were removed and dried. Next, an Ettan DIGE Imager Scanner for 2-D gels was used. The following settings were entered: DIGE-minimal labelling, pixel size $100 \mu \mathrm{m}$ and exposure times Cy2 (0.8 seconds), Cy3 (0.4 seconds) and Cy5 (0.4 seconds). The colours obtained from the scans correspond to the wavelength at which CyDyes are excited at. This was blue for $\mathrm{Cy} 2$, red for $\mathrm{Cy} 5$ and green for 
Cy3. The Ettan DIGE Imager software was used to scan the gels, where the images were edited using the ImageQuant TL program. Before the scanning of a gel took place, the outside of the glass case containing the gel was cleaned using $70 \%$ ethanol. This removed any smears which could obscure protein spots during the scanning process.

\subsubsection{2-D gel analysis and spot picking}

The Decyder ${ }^{\mathrm{TM}} 5.02$ program was used to analyse the scanned 2-D gel images. Filters within the Batch Processor program were pre-set, therefore any spot above the following values would be excluded: Slope: 1.20, Area: 300, Peak Height: 90 and Volume: 8000. Therefore, a minimum ID/C value was set at 1.20 and 0.80 ; meaning any test (ID) protein showing a dysregulation of $20 \%$ from controls was subsequently selected using the Decyder ${ }^{\mathrm{TM}} 5.02$ program. In the Batch Processor program each gel image was uploaded and similarly were the above filter settings and a maximum spot detection number setting of 10000 . The statistics were made using biological variation analysis (BVA). When BVA was used each gel was given a description, therefore which CyDye represented a control or test sample. An independent test with average ratio and paired t-test was run. Observing the different detectable spot differences between control and ID samples meant each spot was observed in turn and decided whether it would be picked depending upon its P-value. All spots that were picked were marked using the Decyder ${ }^{\mathrm{TM}} 5.02$ program, where a print-out of the gel image to the same dimensions of the gel was made. Next the glass plate containing the gel with the best spot resolutions was opened exposing the entire gel surface. The glass plate was then lifted with the gel on its surface and placed directly on-top of the print-out. Spots were picked with a special spot picking pipette with a tip diameter of $1-2 \mathrm{~mm}$. The tip was cleaned with $70 \%$ ethanol between each spot. Samples were excised from the gel without post-staining (silver or Coomassie brilliant blue). After picking the spot, it was transferred to a 96-well multi-titre plate and stored at $-80^{\circ} \mathrm{C}$, until shipment upon dry ice from Ulm to the Proteomics Group of the Max Planck Institute for Experimental Medicine (Hermann-Rein-Straße 3, Göttingen, Germany), for subsequent MALDI-TOF-MS analysis. In addition, a gelplug containing no protein was picked from the same gel. This acted as a negative control.

\subsection{MALDI-TOF-MS}

Samples were stored at $-80^{\circ} \mathrm{C}$ upon arrival from Ulm. Next, automated in-gel digest of the gelplugs by using trypsin was performed using a Genesis ProTeam 150 Advanced Digest (Tecan) robotic system. This was the preparation step of samples prior to MALDI-TOF-MS. Afterwards, $2 \mu 1$ sample extracts were spotted using the same robotic system as described 
above, onto a MALDI-Target which was inserted into a Ultraflex I MALDI-TOF/TOF mass spectrometer (Bruker Daltonics, Germany), where the mass spectra was subsequently analysed via MALDI-TOF-MS. Background signals were removed by subtracting peaks corresponding to peptides produced by trypsin autolysis and were therefore excluded from the database search. BioTools 3.0 software (Bruker Daltonics, Germany), recorded the fragment ion spectra from different precursor ions corresponding to the PMF.

The MS/MS and PMF datasets were processed using BioTools 3.0 software (Bruker Daltonics, Germany) and Mascot 2.0 software. The Swiss-Prot database was used for the main search engine, where the taxonomy was unlimited. The fragment mass tolerance was set at 0.7Da for the MS/MS searches, where for the monoisotopic mass tolerance a setting of $100 \mathrm{ppm}$ was used, where a missed cleavage of one was permitted. For in depth details, please refer to the following journal (Jahn et al., 2006).

All mass spectrometry was performed at the laboratory of Dr. Olaf Jahn at the Proteomics Group of the Max Planck Institute for Experimental Medicine (Hermann-Rein-Straße 3, Göttingen, Germany).

\subsection{Tank Western blotting}

\subsubsection{1-D gel electrophoresis}

Once the protein concentrations of the samples were determined (see section 4.7.4.2.), the volumes required for both $3 \mu \mathrm{g}$ or $50 \mu \mathrm{g}$ protein loads were calculated. Note the $3 \mu \mathrm{g}$ load was used for investigating SIRT2 and myelin markers, where the $50 \mu \mathrm{g}$ protein load was used for investigating $\mathrm{TH}, \mathrm{DAT}$ and ChAT. When loading $50 \mu \mathrm{g}$ protein onto a gel, no pre-dilution of sample was required, only the calculated tissue sample volume was diluted in combination with 1x, 2x and/or 3x 2-Mercaptoethanol (Sigma-Aldrich GmbH, Schnelldorf, Germany) solutions. The $3 \times$ NuPage $^{\circledR}$ LDS Sample buffer $+15 \%$ 2-Mercaptoethanol was used for preparing samples for $50 \mu \mathrm{g}$ protein load, preventing the total sample volume from exceeding the total gel well volume of $20 \mu 1$. If preparing samples for a $3 \mu \mathrm{g}$ protein loaded gel, this meant initially a 1:10 dilution was prepared by adding $2 \mu 1$ of sample and $18 \mu$ of tris-buffer saline (TBS) to a $0.5 \mathrm{ml}$ PCR tube. Afterwards, the calculated volume required for a $3 \mu \mathrm{g}$ or $50 \mu \mathrm{g}$ protein load was pipetted into a separate $0.5 \mathrm{ml}$ PCR tube. Next, an equivalent volume of $2 \mathrm{x}$ NuPage ${ }^{\circledR}$ LDS Sample buffer $+10 \%$ 2-Mercaptoethanol (Sigma-Aldrich GmbH, Schnelldorf, Germany) was prepared and added to the sample within separate eppendorf tubes. Afterwards, the volume was made up to $20 \mu \mathrm{l}$ using 1 x NuPage ${ }^{\circledR}$ LDS Sample buffer $+5 \% 2$ Mercaptoethanol (1:2 diluted $2 \times$ NuPage ${ }^{\circledR}$ LDS Sample buffer in $\mathrm{ddH}_{2} \mathrm{O}$ ). The eppendorfs containing the sample $+2 \mathrm{x} / 1 \mathrm{x}$ NuPage ${ }^{\circledR}$ LDS sample buffers were firstly centrifuged on a 
quick cycle and then afterwards placed onto a $40^{\circ} \mathrm{C}$ preheated eppendorf thermomixer and incubated for 20 minutes. Once the incubation was complete the samples were centrifuged at $15 \times 1000$ rpm for 3 minutes.

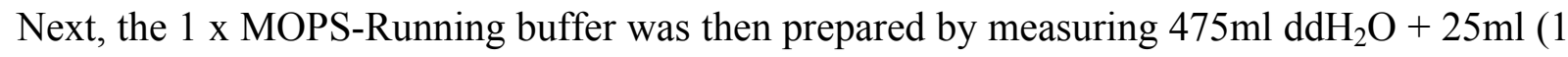
$x$ MOPS Buffer). The precast High-Performance Gels for SDS-PAGE-NuPAGE ${ }^{\circledR}$ Novex $12 \%$ Bis-Tris Gel $1.0 \mathrm{~mm} 12$ well (Invitrogen, Karlsruhe, Germany) were prepared and cleaned using $\mathrm{ddH}_{2} \mathrm{O}$. Once cleaned, the gel was inserted into the XCell SureLock chamber (Invitrogen, Karlsruhe, Germany) and the tank filled with 1 x MOPS-Running buffer. Using a needle and syringe the wells were cleaned using 1 x MOPS-Running buffer. The order of the sample load was pre-determined and a $10 \mu 1$ volume of the molecular marker, SeeBlue ${ }^{\circledR}$ Plus 2 Prestained Standard (1x) (Invitrogen, Karlsruhe, Germany), was used. Empty lanes were filled with $20 \mu \mathrm{l}$ of $1 \times$ NuPage ${ }^{\circledR}$ LDS Sample buffer. Between loading the $20 \mu l$ samples and $10 \mu \mathrm{l}$ molecular marker by using a $25 \mu$ l Hamilton syringe, the syringe was washed 4 times in $1 \mathrm{x}$ MOPS-Running buffer solution. Extra samples were loaded for the initial SIRT2 Western blots. These samples were kindly provided by the following collaborators, Drs Hauke Werner and Olaf Jahn from the Max Planck Institute for Experimental Medicine (Hermann-ReinStraße 3, Göttingen, Germany). The samples were WT brain lysate, protein from proteolipid null mice (PLP) ${ }^{-/}$, WT myelin and $\mathrm{PLP}^{-/-}$myelin. The reason for loading these samples was that they would act as a pre-determined internal sample control helping evaluate the exact bands corresponding to the molecular mass of the SIRT2 protein. Once the samples were loaded into the gels, a $200 \mathrm{~V}, 3 \mathrm{~A}$ and $300 \mathrm{~W}$ setting was used, where with these settings the gel was run for approximately 40-45 minutes. While the gels were running, the Blotting Pad for XCell II Blotting (Invitrogen, Karlsruhe, Germany) was pre-wet in 1 x NuPage $+10 \%$ methanol $(\mathrm{MeOH})$ solution, ensuring no air bubbles were present between each blotting pad. The $1 \mathrm{x} \mathrm{NuPage}+10 \% \mathrm{MeOH}$ is also known as $1 \mathrm{x}$ transfer buffer $+10 \% \mathrm{MeOH}$. The Blotting pads and $1 \times \mathrm{NuPage}+10 \% \mathrm{MeOH}$ solution were left at $4^{\circ} \mathrm{C}$ until the electrophoresis process of samples was complete. If blotting two membranes then $1 \times \mathrm{NuPage}+20 \% \mathrm{MeOH}$ was used instead of $1 \times$ NuPage $+10 \% \mathrm{MeOH}$. Next the Polyvinylidene Difluoride (PVDF) Immobilon-P Transfer Membrane (Millipore GmbH, Schwalbach, Germany) was cut into $8.5 \mathrm{~cm} \times 7.5 \mathrm{~cm}$ pieces, in relation to the $9 \mathrm{~cm} \times 9 \mathrm{~cm}$ gel size. Cutting a corner of the membrane helped identify a particular membrane. The membranes were then pre-wet by placing into $\mathrm{MeOH}$ (Mallinckrodt Baker $\mathrm{GmbH}$, Griesheim, Germany) for 30 seconds, and then rinsed in $\mathrm{ddH}_{2} \mathrm{O}$ prior to transfer into cold $1 \mathrm{x}$ NuPage $+20 \% \mathrm{MeOH}$ solution. Membranes and filter paper were pre-soaked before use in $1 \mathrm{x}$ NuPage $+10 \% \mathrm{MeOH}(1 \mathrm{x}$ transfer buffer $+10 \%$ 
$\mathrm{MeOH}$ ) when transferring one membrane, however when transferring from two membranes a $1 \times$ NuPage $+20 \% \mathrm{MeOH}(1 \times$ transfer buffer $+20 \% \mathrm{MeOH})$ was used.

\subsubsection{Preparation of gel, Western transfer and blotting}

Once the gels were finished, they were removed by separating the gel from the plastic guard. Afterwards, the wells and the bottom of the gel were cut away. One piece of pre-soaked filter paper was placed on top of the gel and trapped air bubbles removed. Thereafter, the presoaked transfer membrane was placed upon the other side of the gel, making sure no air bubbles were present. Another pre-soaked filter paper was placed on top. If running two gels, the two membranes were separated from each other by a monolayer of blotting pad. The top and the bottom of the blotting transfer stack consisted of 2 layers of blotting pad. The stack was then placed into an XCell II Blot Module, which was inserted back into the XCell SureLock chamber and the centre filled with 1 x NuPage $+20 \% \mathrm{MeOH}$. The outside of the chamber was filled with $4^{\circ} \mathrm{C} \mathrm{ddH}_{2} \mathrm{O}$. This setup was run at $30 \mathrm{~V}, 3 \mathrm{~A}$ and $300 \mathrm{~W}$ settings for 1 hour at room temperature. After the transfer was complete, the XCell II Blot Module was opened and the membranes removed with flat-tipped tweezers and placed into a prepared 5\% skimmed milk solution (Blocking solution) in Tris-buffer saline Tween (TBST) $(0.05 \%$ Tween), which was allowed to incubate for 1 hour on a Heidolph Duomax 1030 tilting incubator at $4^{\circ} \mathrm{C}$. Once complete, the box containing the membrane was brought to room temperature. Next, the primary polyclonal antibodies were prepared in $4 \mathrm{ml}$ of $5 \%$ skimmed milk solution. The primary antibodies used were, 1:2000 Rabbit anti-SIRT2 (H-95) (Santa Cruz Biotechnology, Inc. Heidelberg, Germany), 1:700 Goat Anti-ChAT, 1:700 Sheep AntiTH and 1:700 Rabbit Anti-DAT, the latter 3 antibodies were purchased from Millipore GmbH (Millipore GmbH, Schwalbach, Germany). The following custom made monoclonal antibodies were also used, Mouse Anti-MOG (8-18C5) (Chris Linnington Batch) and the Rabbit Anti-PLP/DM20 (A431) (Olga Shakhova Batch). These antibodies were both kindly provided by Dr Hauke Werner (Max Planck Institute for Experimental Medicine, Department of Neurogenetics, Göttingen) and were used at the following concentrations, 1:2500 and 1:5000, respectively. Determining the protein load for each lane, meant the use of the following house-keeping gene antibodies, 1:5000 Mouse Anti-Actin Monoclonal Antibody Clone AC-40 (Sigma-Aldrich Chemie GmbH, Steinheim, Germany) and 1:5000 Mouse AntiGAPDH (Stressgen Bioreagents, Biomol GmbH, Hamburg, Germany). After preparing the $4 \mathrm{ml}$ primary antibody solutions in $50 \mathrm{ml}$ falcon tubes the membranes were neatly folded so that the protein-side faced inwards. Afterwards, the tubes (including antibody and membranes) were then incubated at $4^{\circ} \mathrm{C}$ and left overnight on an Assistant 348 rolling incubator. 
Once the primary antibody incubation time was complete, the membranes were removed from the $50 \mathrm{ml}$ falcon tubes and placed into separate boxes filled with TBST and were left to incubate at room temperature for 15 minutes on a GFL 3015 shaking incubator, set to a low revolution speed. This wash step was repeated 3 times to reduce background staining. Each wash step involved removing the old TBST and replacing with fresh solution. The secondary antibody used for SIRT2, DAT or PLP, was a Goat Anti-Rabbit IgG, HRP conjugated (DIANOVA GmbH, Hamburg, Germany) antibody, which was diluted in $5 \mathrm{ml}$ of 5\% skimmed milk solution to $1: 10000$ for SIRT2 and 1:5000 for the other antibodies. MOG, Actin and GAPDH were incubated with Goat Anti-Mouse IgG horseradish peroxidase (HRP) conjugate antibody (DIANOVA GmbH, Hamburg, Germany), which was diluted to 1:5000 in $5 \mathrm{ml}$ of 5\% skimmed milk solution. Finally, ChAT and TH were incubated at a concentration of 1:5000 in $5 \mathrm{ml}$ of $5 \%$ skimmed milk solution, with Rabbit Anti-Goat IgG, HRP conjugate (SigmaAldrich GmbH, Schnelldorf, Germany) and Donkey Anti-Sheep IgG, HRP conjugate (DIANOVA GmbH, Hamburg, Germany) antibodies, respectively. The membranes were then placed into separate $50 \mathrm{ml}$ falcon tubes containing the diluted secondary antibody and incubated on an Assistant 348 rolling incubator for 1 hour at room temperature. Afterwards, the membranes were washed using a $3 \times 15$ minute wash procedure in TBST (see above). Between the second and third wash the bands in the marker lane were marked upon the protein side of the membrane by using a fine-tipped black permanent marker pen, where the orange bands $(19 \mathrm{kDa}$ and $97 \mathrm{kDa})$ of the SeeBlue ${ }^{\circledR}$ Plus 2 Prestained Standard $(1 \mathrm{x})$ were marked with a cross. After the TBST solution was drained from the membranes, they were placed between two clear plastic sheets, which were secured down in a film cassette. This helped keep the membranes dust free during transport prior to exposure.

\subsubsection{Developing procedure for membranes}

The membranes were transported in a film cassette to the developing room. The membranes were placed flat, i.e. protein side up upon a clean plastic sheet. Per membrane, a volume of $2 \mathrm{ml}$ Western Lighting ${ }^{\mathrm{TM}}$ Chemiluminescence Reagent Plus oxidizing reagent $+2 \mathrm{ml}$ Western Lighting $^{\mathrm{TM}}$ Chemiluminescence Reagent Plus Enhanced Luminol Reagent (PerkinElmer Optoelectronics $\mathrm{GmbH} \&$ Co. KG, Wiesbaden, Germany) was required. This solution was pipetted into a $50 \mathrm{ml}$ falcon tube and mixed, where afterwards it was pipetted over the surface of the membrane. The solution was left upon the membranes for 1 minute at room temperature. Afterwards, this solution was drained off and the membranes laid flat between plastic sheets in the film cassette. Next, the cassette was closed and the lights switched off. Amersham Hyperfilm ${ }^{\mathrm{TM}}$ ECL high performance chemiluminescence film $(18 \times 24 \mathrm{~cm})$ was earmarked for orientation at the top left corner and then placed above the plastic sheet holding the 
membrane. The timer was started and left for varying exposure times until band density was optimised, initially 1 minute time exposure was used. Once the incubation time was complete the film was removed from the cassette and placed into a Kodak X-OMAT 1000 Processor (Kodak GmbH, Stuttgart, Germany). Once the film was developed the lights were switched on and the developed film was laid ontop of the membrane in the cassette where the marker lane was labelled on to the film. Afterwards, the membranes were washed 2 x 5 minutes in 1 x TBS (without Tween 20) solution, and from this point could be maintained in $1 \mathrm{x}$ TBS for a maximum of 4 weeks at $4^{\circ} \mathrm{C}$. Storage of membranes for longer periods requires sealing the membrane in a clean plastic bag with $1 \mathrm{x}$ TBS solution and storing at $4^{\circ} \mathrm{C}$.

\subsubsection{Membrane stripping}

The membranes is removed from $1 \times$ TBS solution at $4^{\circ} \mathrm{C}$ and washed $2 \times 10$ minutes in plastic boxes containing TBST solution. The boxe containing the membrane was left on a shaking incubator at room temperature for the duration of the wash. Afterwards, the membrane was removed from the TBST solution and placed into flat glass beakers, where Strip-Buffer was added and allowed to incubate for $2 \times 15$ minutes at room temperature on a shaking incubator. Next the membrane was returned to a plastic box with fresh TBST solution and the $2 \times 10$ minute wash step was repeated at room temperature. The membrane was then blocked using 5\% skimmed milk solution for 30 minutes at room temperature, prior to repeated exposure to the corresponding secondary antibody for 30 minutes at room temperature. Subsequently, the membrane was washed in TBST for 3 x 5 minutes.

The membrane was developed and subsequently exposed to hyperfilm for 3 minutes to check for the presence of a high background (see above section 4.10.3.). Once no bands and no high background were observed, the membrane was re-used for blotting. The procedure was to return the membrane back to TBST solution and use a 3 x 5 minute wash step at room temperature followed by a 1 hour block at $4^{\circ} \mathrm{C}$ using $5 \%$ skimmed milk solution + TBST (Blocking solution), before the primary antibody was applied. The following blotting procedure of both primary and secondary antibodies is the same as previously described (section 4.10.2.).

\subsection{Quantifying from Western blots}

Use of quantitative Western blotting has aided in evaluating the differences in protein expression identified previously in the 2-D DIGE/MALDI-TOF-MS experiments.

Once the membranes were exposed and hyperfilm developed, the exposed films were scanned at 400dpi and stored onto a hard drive. The following protocol was used for every 
image. Images were opened in turn using ImageJ 1.32j (NIH software, Bethesda, MD, USA) and converted to an 8-bit format. Prior to analysis the background on every image was reduced by a pre-setting value of 50 . The parameters, area and mean grey value (density) were selected. Afterwards, the scale of pixels/cm was set. Images were inverted and the bands quantified using an enclosed box feature in the program. The displayed values from the Western blots are the mean grey value (density) of the band. The house keeping gene markers, Actin and GAPDH proved critical as an internal loading control, where the absolute values of protein band densities where represented in relation to either Actin of GAPDH. This combined value was subsequently used in the analysis.

\subsection{2-D IEF/SDS-PAGE}

This procedure was similar to the 2-D DIGE experiments as described previously (section 4.8.3.). Firstly, the 1-D electrophoresis (see section 4.8.1.) was performed only with one strip corresponding to one sample, therefore one animal. Furthermore, a short $7 \mathrm{~cm}$ strip was used to separate the samples according to a $\mathrm{pH}$ range from 4-7. In addition, as only one sample resides within each strip, meant there was no requirement for the use of CyDyes, instead a peroxidise method was employed. Secondly, the strip was cut according to size and layed on top of the $12.5 \%$ SDS gel prior to SDS-PAGE. Adjacent to the SDS-PAGE, a 1-D molecular marker was loaded. All 2-D IEF/SDS-PAGE blots were performed at the laboratory of our collaborator, Professor Dr. Med. Markus Otto (Neurology Group, Centre for Clinical Research, Helmholzstraße 8/1, Ulm, Germany).

\subsection{Statistics}

\subsubsection{General physiology, immunohistochemistry and pain test data}

Firstly, Bartlett tests were performed using MINITAB ${ }^{\circledR} 14$ (Minitab Inc, Friedrichsdorf, Germany) to determine whether the dataset was normally distributed, which was true for all datasets. A univariate ANOVA was used to test the effect of diet upon the forelimb grip strength. A two-factorial ANOVA was performed to test whether iron free diet and the duration of iron free diet had an effect upon one of the following: the plasma-iron concentration, haematocrit, haemoglobin, the hot-plate test reaction time, ChAT-IR terminal density, ChAT-IR motoneuron area. A three-factorial ANOVA was performed to test whether iron free diet, the duration of iron free diet and the dorsal horn side had an effect upon the number of ipsilateral/contralateral c-Fos-IR cells. A two-factorial ANOVA for repeated measures was used to investigate the effect iron free diet and duration of diet had upon the animal weight. In addition a three-factorial ANOVA was performed to test whether iron free 
diet, the duration of iron free diet and the time as the repeated measure representing the different time-points of the formalin-test, had an effect upon the response score. Post hoc tests (Student's t-test, level of significance $P<0.05$ ) were performed to compare the values of both diet groups at different time bins. The ANOVAs and Student's t-tests were performed using SPSS 17.0.0 (SPSS Software, München, Germany). The level of significance for rejecting the null hypothesis was set at $P<0.05$ for all statistical calculations. All data is presented as mean \pm SEM.

\subsubsection{Voluntary wheel-running data}

ANOVA tests for repeated measures were performed for 1 hour and when stated 2 hour voluntary wheel-running data to test whether iron free diet and the duration of iron free diet had an effect upon the Distance, $\mathrm{V}_{\max }, \mathrm{N}_{\text {run }}, \mathrm{T}_{\text {run }}$, Distance/ $\mathrm{N}_{\text {run }}$, Percentage of max $\mathrm{N}_{\text {run }}$ and the Percentage of max $T_{\text {run. }}$ The diet, strain and duration were set as between subject factors with time as the within subject variable. Furthermore, the aforementioned parameters were investigated during the dark phase only $(19: 00 \mathrm{hr}-07: 00 \mathrm{hr})$ for the interaction effects of diet, strain and duration with time in a four-factorial ANOVA. Post hoc tests (Student's t-test, level of significance $P<0.05$ ) were performed to compare the values of diet groups, in particular at defined time epochs $(03: 00 \mathrm{hr}-04: 00 \mathrm{hr}$ and 06:00hr-07:00hr). The ANOVAs and Student's ttests were performed using SPSS 17.0.0 (SPSS Software, München, Germany). All data is presented as mean \pm SEM. 


\section{References}

Accili, D., Fishburn, C.S., Drago, J., Steiner, H., Lachowicz, J.E., Park, B.H., Gauda, E.B., Lee, E.J., Cool, M.H., Sibley, D.R., Gerfen, C.R., Westphal, H., Fuchs, S., 1996. A targeted mutation of the $\mathrm{D} 3$ dopamine receptor gene is associated with hyperactivity in mice. Proc Natl Acad Sci U S A. 93, 1945-1949.

Akpinar, S., 1982. Treatment of restless legs syndrome with levodopa plus benserazide. Arch Neurol. 39, 739.

Allen, R.P., Earley, C.J., 1996. Augmentation of the restless legs syndrome with carbidopa/levodopa. Sleep. 19, 205-213.

Allen, R.P., Earley, C.J., 2000. Defining the phenotype of the restless legs syndrome (RLS) using age-of-symptom-onset. Sleep Med. 1, 11-19.

Allen, R.P., Earley, C.J., 2001. Restless legs syndrome: a review of clinical and pathophysiologic features. J Clin Neurophysiol. 18, 128-147.

Allen, R.P., Picchietti, D., Hening, W.A., Trenkwalder, C., Walters, A.S., Montplaisi, J., 2003. Restless legs syndrome: diagnostic criteria, special considerations, and epidemiology. A report from the restless legs syndrome diagnosis and epidemiology workshop at the National Institutes of Health. Sleep Med. 4, 101-119.

Allen, R.P., Walters, A.S., Montplaisir, J., Hening, W., Myers, A., Bell, T.J., Ferini-Strambi, L., 2005. Restless legs syndrome prevalence and impact: REST general population study. Arch Intern Med. 165, 1286-1292.

Ashkenazi, R., Ben-Shachar, D., Youdim, M.B., 1982. Nutritional iron and dopamine binding sites in the rat brain. Pharmacol Biochem Behav. 17 Suppl 1, 43-47.

Austin, N.E., Baldwin, S.J., Cutler, L., Deeks, N., Kelly, P.J., Nash, M., Shardlow, C.E., Stemp, G., Thewlis, K., Ayrton, A., Jeffrey, P., 2001. Pharmacokinetics of the novel, high-affinity and selective dopamine D3 receptor antagonist SB-277011 in rat, dog and monkey: in vitro/in vivo correlation and the role of aldehyde oxidase. Xenobiotica. 31, 677-686.

Baier, P.C., Ondo, W.G., Winkelmann, J., 2007. Animal studies in restless legs syndrome. Mov Disord. 22 Suppl 18, S459-465.

Baier, P.C., Winkelmann, J., Hohne, A., Lancel, M., Trenkwalder, C., 2002. Assessment of spontaneously occurring periodic limb movements in sleep in the rat. J Neurol Sci. 198, 71-77.

Baik, J.H., Picetti, R., Saiardi, A., Thiriet, G., Dierich, A., Depaulis, A., Le Meur, M., Borrelli, E., 1995. Parkinsonian-like locomotor impairment in mice lacking dopamine D2 receptors. Nature. 377, 424-428.

Barik, S., de Beaurepaire, R., 2005. Dopamine D3 modulation of locomotor activity and sleep in the nucleus accumbens and in lobules 9 and 10 of the cerebellum in the rat. Prog Neuropsychopharmacol Biol Psychiatry. 29, 718-726.

Barriere, G., Cazalets, J.R., Bioulac, B., Tison, F., Ghorayeb, I., 2005. The restless legs syndrome. Prog Neurobiol. 77, 139-165.

Barriere, G., Mellen, N., Cazalets, J.R., 2004. Neuromodulation of the locomotor network by dopamine in the isolated spinal cord of newborn rat. Eur J Neurosci. 19, 1325-1335.

Basbaum, A.I., Fields, H.L., 1984. Endogenous pain control systems: brainstem spinal pathways and endorphin circuitry. Annu Rev Neurosci. 7, 309-338.

Beard, J., Erikson, K.M., Jones, B.C., 2003a. Neonatal iron deficiency results in irreversible changes in dopamine function in rats. J Nutr. 133, 1174-1179.

Beard, J.L., Chen, Q., Connor, J., Jones, B.C., 1994. Altered monamine metabolism in caudate-putamen of iron-deficient rats. Pharmacol Biochem Behav. 48, 621-624.

Beard, J.L., Connor, J.R., 2003. Iron status and neural functioning. Annu Rev Nutr. 23, 41-58.

Beard, J.L., Connor, J.R., Jones, B.C., 1993. Iron in the brain. Nutr Rev. 51, 157-170.

Beard, J.L., Erikson, K.M., Jones, B.C., 2002. Neurobehavioral analysis of developmental iron deficiency in rats. Behav Brain Res. 134, 517-524. 
Beard, J.L., Unger, E.L., Bianco, L.E., Paul, T., Rundle, S.E., Jones, B.C., 2007. Early postnatal iron repletion overcomes lasting effects of gestational iron deficiency in rats. J Nutr. 137, 1176-1182.

Beard, J.L., Wiesinger, J.A., Connor, J.R., 2003b. Pre- and postweaning iron deficiency alters myelination in Sprague-Dawley rats. Dev Neurosci. 25, 308-315.

Beard, J.L., Wiesinger, J.A., Li, N., Connor, J.R., 2005. Brain iron uptake in hypotransferrinemic mice: influence of systemic iron status. J Neurosci Res. 79, 254261.

Beard, J.L., Zhan, C.S., Brigham, D.E., 1995. Growth in iron-deficient rats. Proc Soc Exp Biol Med. 209, 65-72.

Ben-Sreti, M.M., Gonzalez, J.P., Sewell, R.D., 1983. Differential effects of SKF 38393 and LY 141865 on nociception and morphine analgesia. Life Sci. 33 Suppl 1, 665-668.

Benarroch, E.E., 2008. Descending monoaminergic pain modulation: bidirectional control and clinical relevance. Neurology. 71, 217-221.

Berger, B., Verney, C., Gaspar, P., Febvret, A., 1985. Transient expression of tyrosine hydroxylase immunoreactivity in some neurons of the rat neocortex during postnatal development. Brain Res. 355, 141-144.

Berger, K., von Eckardstein, A., Trenkwalder, C., Rothdach, A., Junker, R., Weiland, S.K., 2002. Iron metabolism and the risk of restless legs syndrome in an elderly general population--the MEMO-Study. J Neurol. 249, 1195-1199.

Bifulco, M., Laezza, C., Stingo, S., Wolff, J., 2002. 2',3'-Cyclic nucleotide 3'phosphodiesterase: a membrane-bound, microtubule-associated protein and membrane anchor for tubulin. Proc Natl Acad Sci U S A. 99, 1807-1812.

Billat, V.L., Mouisel, E., Roblot, N., Melki, J., 2005. Inter- and intrastrain variation in mouse critical running speed. J Appl Physiol. 98, 1258-1263.

Bishop, G.M., Robinson, S.R., Liu, Q., Perry, G., Atwood, C.S., Smith, M.A., 2002. Iron: a pathological mediator of Alzheimer disease? Dev Neurosci. 24, 184-187.

Blusztajn, J.K., Wurtman, R.J., 1983. Choline and cholinergic neurons. Science. 221, 614-620.

Bonati, M.T., Ferini-Strambi, L., Aridon, P., Oldani, A., Zucconi, M., Casari, G., 2003. Autosomal dominant restless legs syndrome maps on chromosome 14q. Brain. 126, 1485-1492.

Bonnot, A., Whelan, P.J., Mentis, G.Z., O'Donovan, M.J., 2002. Locomotor-like activity generated by the neonatal mouse spinal cord. Brain Res Brain Res Rev. 40, 141-151.

Bourque, S.L., Iqbal, U., Reynolds, J.N., Adams, M.A., Nakatsu, K., 2008. Perinatal iron deficiency affects locomotor behavior and water maze performance in adult male and female rats. J Nutr. 138, 931-937.

Brandon, E.P., Lin, W., D'Amour, K.A., Pizzo, D.P., Dominguez, B., Sugiura, Y., Thode, S., Ko, C.P., Thal, L.J., Gage, F.H., Lee, K.F., 2003. Aberrant patterning of neuromuscular synapses in choline acetyltransferase-deficient mice. J Neurosci. 23, 539-549.

Bucher, S.F., Seelos, K.C., Oertel, W.H., Reiser, M., Trenkwalder, C., 1997. Cerebral generators involved in the pathogenesis of the restless legs syndrome. Ann Neurol. 41, 639-645.

Burhans, M.S., Dailey, C., Beard, Z., Wiesinger, J., Murray-Kolb, L., Jones, B.C., Beard, J.L., 2005. Iron deficiency: differential effects on monoamine transporters. Nutr Neurosci. $8,31-38$.

Burnes, L.A., Kolker, S.J., Danielson, J.F., Walder, R.Y., Sluka, K.A., 2008. Enhanced muscle fatigue occurs in male but not female ASIC3-/- mice. Am J Physiol Regul Integr Comp Physiol. 294, R1347-1355.

Bussel, B., Roby-Brami, A., Azouvi, P., Biraben, A., Yakovleff, A., Held, J.P., 1988. Myoclonus in a patient with spinal cord transection. Possible involvement of the spinal stepping generator. Brain. 111 ( Pt 5), 1235-1245. 
Butt, S.J., Lebret, J.M., Kiehn, O., 2002. Organization of left-right coordination in the mammalian locomotor network. Brain Res Brain Res Rev. 40, 107-117.

Calne, D.B., Plotkin, C., Williams, A.C., Nutt, J.G., Neophytides, A., Teychenne, P.F., 1978. Long-term treatment of parkinsonism with bromocriptine. Lancet. 1, 735-738.

Carr, K.D., Yamamoto, N., Omura, M., Cabeza de Vaca, S., Krahne, L., 2002. Effects of the $\mathrm{D}$ (3) dopamine receptor antagonist, U99194A, on brain stimulation and damphetamine reward, motor activity, and c-fos expression in ad libitum fed and foodrestricted rats. Psychopharmacology (Berl). 163, 76-84.

Cazalets, J.R., Bertrand, S., 2000. Coupling between lumbar and sacral motor networks in the neonatal rat spinal cord. Eur J Neurosci. 12, 2993-3002.

Cazalets, J.R., Borde, M., Clarac, F., 1995. Localization and organization of the central pattern generator for hindlimb locomotion in newborn rat. J Neurosci. 15, 4943-4951.

Cazalets, J.R., Sqalli-Houssaini, Y., Clarac, F., 1992. Activation of the central pattern generators for locomotion by serotonin and excitatory amino acids in neonatal rat. $\mathrm{J}$ Physiol. 455, 187-204.

Cho, S.S., Shin, D.H., Lee, K.H., Hwang, D.H., Chang, K.Y., 1998. Localization of transferrin binding protein in relation to iron, ferritin, and transferrin receptors in the chicken cerebellum. Brain Res. 794, 174-178.

Ciliax, B.J., Heilman, C., Demchyshyn, L.L., Pristupa, Z.B., Ince, E., Hersch, S.M., Niznik, H.B., Levey, A.I., 1995. The dopamine transporter: immunochemical characterization and localization in brain. J Neurosci. 15, 1714-1723.

Clemens, S., Hochman, S., 2004. Conversion of the modulatory actions of dopamine on spinal reflexes from depression to facilitation in D3 receptor knock-out mice. J Neurosci. 24, 11337-11345.

Clemens, S., Rye, D., Hochman, S., 2006. Restless legs syndrome: revisiting the dopamine hypothesis from the spinal cord perspective. Neurology. 67, 125-130.

Clemens, S., Sawchuk, M.A., Hochman, S., 2005. Reversal of the circadian expression of tyrosine-hydroxylase but not nitric oxide synthase levels in the spinal cord of dopamine D3 receptor knockout mice. Neuroscience. 133, 353-357.

Clifford, J.J., Waddington, J.L., 1998. Heterogeneity of behavioural profile between three new putative selective D3 dopamine receptor antagonists using an ethologically based approach. Psychopharmacology (Berl). 136, 284-290.

Collado-Seidel, V., Kazenwadel, J., Wetter, T.C., Kohnen, R., Winkelmann, J., Selzer, R., Oertel, W.H., Trenkwalder, C., 1999. A controlled study of additional sr-L-dopa in Ldopa-responsive restless legs syndrome with late-night symptoms. Neurology. 52, 285290.

Connor, J.R., 1994. Iron acquisition and expression of iron regulatory proteins in the developing brain: manipulation by ethanol exposure, iron deprivation and cellular dysfunction. Dev Neurosci. 16, 233-247.

Connor, J.R., Boyer, P.J., Menzies, S.L., Dellinger, B., Allen, R.P., Ondo, W.G., Earley, C.J., 2003. Neuropathological examination suggests impaired brain iron acquisition in restless legs syndrome. Neurology. 61, 304-309.

Connor, J.R., Menzies, S.L., 1990. Altered cellular distribution of iron in the central nervous system of myelin deficient rats. Neuroscience. 34, 265-271.

Connor, J.R., Menzies, S.L., 1995. Cellular management of iron in the brain. J Neurol Sci. 134 Suppl, 33-44.

Cook, J.D., Baynes, R.D., Skikne, B.S.,, 1992. Iron deficiency and the measurement of iron status. Nutrition Research Reviews. 5, 189-202.

Cook, J.D., Skikne, B.S., 1989. Iron deficiency: definition and diagnosis. J Intern Med. 226, 349-355.

Cornish, E.R., Mrosovsky, N., 1965. Activity during food deprivation and satiation of six species of rodent. Anim Behav. 13, 242-248. 
Cowley, K.C., Schmidt, B.J., 1994. A comparison of motor patterns induced by N-methyl-Daspartate, acetylcholine and serotonin in the in vitro neonatal rat spinal cord. Neurosci Lett. 171, 147-150.

Cowley, K.C., Schmidt, B.J., 1997. Regional distribution of the locomotor pattern-generating network in the neonatal rat spinal cord. J Neurophysiol. 77, 247-259.

Dallman, P.R., 1986. Biochemical basis for the manifestations of iron deficiency. Annu Rev Nutr. 6, 13-40.

Dallman, P.R., Siimes, M.A., Manies, E.C., 1975. Brain iron: persistent deficiency following short-term iron deprivation in the young rat. Br J Haematol. 31, 209-215.

Davies, K.J., Donovan, C.M., Refino, C.J., Brooks, G.A., Packer, L., Dallman, P.R., 1984. Distinguishing effects of anemia and muscle iron deficiency on exercise bioenergetics in the rat. Am J Physiol. 246, E535-543.

de Mello, M.T., Lauro, F.A., Silva, A.C., Tufik, S., 1996. Incidence of periodic leg movements and of the restless legs syndrome during sleep following acute physical activity in spinal cord injury subjects. Spinal Cord. 34, 294-296.

Dean, T., Jr., Allen, R.P., O'Donnell, C.P., Earley, C.J., 2006. The effects of dietary iron deprivation on murine circadian sleep architecture. Sleep Med. 7, 634-640.

Dickenson, A.H., Sullivan, A.F., 1987. Subcutaneous formalin-induced activity of dorsal horn neurones in the rat: differential response to an intrathecal opiate administered pre or post formalin. Pain. 30, 349-360.

Dickinson, S.D., Sabeti, J., Larson, G.A., Giardina, K., Rubinstein, M., Kelly, M.A., Grandy, D.K., Low, M.J., Gerhardt, G.A., Zahniser, N.R., 1999. Dopamine D2 receptordeficient mice exhibit decreased dopamine transporter function but no changes in dopamine release in dorsal striatum. J Neurochem. 72, 148-156.

Dobbing, J., 1990. Boyd Orr memorial lecture. Early nutrition and later achievement. Proc Nutr Soc. 49, 103-118.

Dorazil-Dudzik, M., Mika, J., Schafer, M.K., Li, Y., Obara, I., Wordliczek, J., Przewlocka, B., 2004. The effects of local pentoxifylline and propentofylline treatment on formalininduced pain and tumor necrosis factor-alpha messenger RNA levels in the inflamed tissue of the rat paw. Anesth Analg. 98, 1566-1573, table of contents.

Dubuisson, D., Dennis, S.G., 1977. The formalin test: a quantitative study of the analgesic effects of morphine, meperidine, and brain stem stimulation in rats and cats. Pain. 4, 161-174.

Earley, C.J., Allen, R.P., 1996. Pergolide and carbidopa/levodopa treatment of the restless legs syndrome and periodic leg movements in sleep in a consecutive series of patients. Sleep. 19, 801-810.

Earley, C.J., Connor, J.R., Beard, J.L., Malecki, E.A., Epstein, D.K., Allen, R.P., 2000. Abnormalities in CSF concentrations of ferritin and transferrin in restless legs syndrome. Neurology. 54, 1698-1700.

Earley, C.J., Heckler, D., Allen, R.P., 2004. The treatment of restless legs syndrome with intravenous iron dextran. Sleep Med. 5, 231-235.

Eddy, N.B., Leimbach, D., 1953. Synthetic analgesics. II. Dithienylbutenyl- and dithienylbutylamines. J Pharmacol Exp Ther. 107, 385-393.

Eiden, L.E., 1998. The cholinergic gene locus. J Neurochem. 70, 2227-2240.

Ekbom, K.A., 1944. Astenia crurum paraesthetica ('Irritable legs'). Acta Med Scand. 118, 197-209.

Ekbom, K.A., 1945. Restless legs syndrome: a clinical study. Acta Med Scand. Supplementum $158,4-122$.

Ekbom, K.A., 1960. Restless legs syndrome. Neurology. 10, 868-873.

Erikson, K.M., Jones, B.C., Beard, J.L., 2000. Iron deficiency alters dopamine transporter functioning in rat striatum. J Nutr. 130, 2831-2837.

Erikson, K.M., Jones, B.C., Hess, E.J., Zhang, Q., Beard, J.L., 2001. Iron deficiency decreases dopamine D1 and D2 receptors in rat brain. Pharmacol Biochem Behav. 69, 409-418. 
Erikson, K.M., Pinero, D.J., Connor, J.R., Beard, J.L., 1997. Regional brain iron, ferritin and transferrin concentrations during iron deficiency and iron repletion in developing rats. J Nutr. 127, 2030-2038.

Esteves, A.M., de Mello, M.T., Lancellotti, C.L., Natal, C.L., Tufik, S., 2004. Occurrence of limb movement during sleep in rats with spinal cord injury. Brain Res. 1017, 32-38.

Faucheux, B.A., Nillesse, N., Damier, P., Spik, G., Mouatt-Prigent, A., Pierce, A., Leveugle, B., Kubis, N., Hauw, J.J., Agid, Y., et al., 1995. Expression of lactoferrin receptors is increased in the mesencephalon of patients with Parkinson disease. Proc Natl Acad Sci U S A. 92, 9603-9607.

Ferguson, S.M., Savchenko, V., Apparsundaram, S., Zwick, M., Wright, J., Heilman, C.J., Yi, H., Levey, A.I., Blakely, R.D., 2003. Vesicular localization and activity-dependent trafficking of presynaptic choline transporters. J Neurosci. 23, 9697-9709.

Festing, M.F., Greenwood, R., 1976. Home-cage wheel activity recording in mice. Lab Anim. 10, 81-85.

Finger, F.W., 1969. Estrus and general activity in the rat. J Comp Physiol Psychol. 68, 461466.

Frolich, M.A., Price, D.D., Robinson, M.E., Shuster, J.J., Theriaque, D.W., Heft, M.W., 2005. The effect of propofol on thermal pain perception. Anesth Analg. 100, 481-486.

Frye, R.A., 1999. Characterization of five human cDNAs with homology to the yeast SIR2 gene: Sir2-like proteins (sirtuins) metabolize NAD and may have protein ADPribosyltransferase activity. Biochem Biophys Res Commun. 260, 273-279.

Frye, R.A., 2000. Phylogenetic classification of prokaryotic and eukaryotic Sir2-like proteins. Biochem Biophys Res Commun. 273, 793-798.

Fulco, M., Schiltz, R.L., Iezzi, S., King, M.T., Zhao, P., Kashiwaya, Y., Hoffman, E., Veech, R.L., Sartorelli, V., 2003. Sir2 regulates skeletal muscle differentiation as a potential sensor of the redox state. Mol Cell. 12, 51-62.

Gabra, B.H., Sirois, P., 2005. Hyperalgesia in non-obese diabetic (NOD) mice: a role for the inducible bradykinin B1 receptor. Eur J Pharmacol. 514, 61-67.

Gambling, L., Andersen, H.S., Czopek, A., Wojciak, R., Krejpcio, Z., McArdle, H.J., 2004. Effect of timing of iron supplementation on maternal and neonatal growth and iron status of iron-deficient pregnant rats. J Physiol. 561, 195-203.

Garcia-Borreguero, D., Allen, R.P., Kohnen, R., Hogl, B., Trenkwalder, C., Oertel, W., Hening, W.A., Paulus, W., Rye, D., Walters, A., Winkelmann, J., Earley, C.J., 2007. Diagnostic standards for dopaminergic augmentation of restless legs syndrome: report from a World Association of Sleep Medicine-International Restless Legs Syndrome Study Group consensus conference at the Max Planck Institute. Sleep Med. 8, 520-530.

Garcia, S.J., Gellein, K., Syversen, T., Aschner, M., 2007. Iron deficient and manganese supplemented diets alter metals and transporters in the developing rat brain. Toxicol Sci. 95, 205-214.

Gebhart, G.F., 2004. Descending modulation of pain. Neurosci Biobehav Rev. 27, 729-737.

Gerlach, M., Ben-Shachar, D., Riederer, P., Youdim, M.B., 1994. Altered brain metabolism of iron as a cause of neurodegenerative diseases? J Neurochem. 63, 793-807.

Godau, J., Schweitzer, K.J., Liepelt, I., Gerloff, C., Berg, D., 2007. Substantia nigra hypoechogenicity: definition and findings in restless legs syndrome. Mov Disord. 22, 187-192.

Gold, R., Lenox, R.H., 1995. Is there a rationale for iron supplementation in the treatment of akathisia? A review of the evidence. J Clin Psychiatry. 56, 476-483.

Green, K.N., Steffan, J.S., Martinez-Coria, H., Sun, X., Schreiber, S.S., Thompson, L.M., LaFerla, F.M., 2008. Nicotinamide restores cognition in Alzheimer's disease transgenic mice via a mechanism involving sirtuin inhibition and selective reduction of Thr231phosphotau. J Neurosci. 28, 11500-11510. 
Hanbury, R., Ling, Z.D., Wuu, J., Kordower, J.H., 2003. GFAP knockout mice have increased levels of GDNF that protect striatal neurons from metabolic and excitotoxic insults. $\mathrm{J}$ Comp Neurol. 461, 307-316.

Heapy, C.G., Jamieson, A., Russel, N.J.W., 1987. Afferent C-fiber and A-delta activity in models of inflammation. British Journal of Pharmacology. 90, 146p.

Hogl, B., Kiechl, S., Willeit, J., Saletu, M., Frauscher, B., Seppi, K., Muller, J., Rungger, G., Gasperi, A., Wenning, G., Poewe, W., 2005. Restless legs syndrome: a communitybased study of prevalence, severity, and risk factors. Neurology. 64, 1920-1924.

Honda, K., Harada, A., Takano, Y., Kamiya, H., 2000. Involvement of M3 muscarinic receptors of the spinal cord in formalin-induced nociception in mice. Brain Res. 859, 38-44.

Hong, Z., Wang, G., Gu, J., Pan, J., Bai, L., Zhang, S., Chen, S.D., 2007. Tripchlorolide protects against MPTP-induced neurotoxicity in C57BL/6 mice. Eur J Neurosci. 26, $1500-1508$.

Huie, J.M., Sharma, R.P., Coulombe, R.A., Jr., 1989. Diurnal alterations of catecholamines, indoleamines and their metabolites in specific brain regions of the mouse. Comp Biochem Physiol C. 94, 575-579.

Hunt, J.R., Zito, C.A., Erjavec, J., Johnson, L.K., 1994. Severe or marginal iron deficiency affects spontaneous physical activity in rats. Am J Clin Nutr. 59, 413-418.

Hunter, J.C., Woodburn, V.L., Durieux, C., Pettersson, E.K., Poat, J.A., Hughes, J., 1995. cfos antisense oligodeoxynucleotide increases formalin-induced nociception and regulates preprodynorphin expression. Neuroscience. 65, 485-492.

Iacovitti, L., Lee, J., Joh, T.H., Reis, D.J., 1987. Expression of tyrosine hydroxylase in neurons of cultured cerebral cortex: evidence for phenotypic plasticity in neurons of the CNS. J Neurosci. 7, 1264-1270.

Ikeda, H., Stark, J., Fischer, H., Wagner, M., Drdla, R., Jager, T., Sandkuhler, J., 2006. Synaptic amplifier of inflammatory pain in the spinal dorsal horn. Science. 312, 16591662.

Ishizaki, K., Sasaki, M., Karasawa, S., Obata, H., Nara, T., Goto, F., 1999. The effect of intrathecal magnesium sulphate on nociception in rat acute pain models. Anaesthesia. 54, 241-246.

Isobe, Y., Nishino, H., 2001. Circadian rhythm of drinking and running-wheel activity in rats with 6-hydroxydopamine lesions of the ventral tegmental area. Brain Res. 899, 187 192.

Jahn, O., Hesse, D., Reinelt, M., Kratzin, H.D., 2006. Technical innovations for the automated identification of gel-separated proteins by MALDI-TOF mass spectrometry. Anal Bioanal Chem. 386, 92-103.

Ji, R.R., Rupp, F., 1997. Phosphorylation of transcription factor CREB in rat spinal cord after formalin-induced hyperalgesia: relationship to c-fos induction. J Neurosci. 17, 17761785.

Juvin, L., Simmers, J., Morin, D., 2005. Propriospinal circuitry underlying interlimb coordination in mammalian quadrupedal locomotion. J Neurosci. 25, 6025-6035.

Kalen, P., Skagerberg, G., Lindvall, O., 1988. Projections from the ventral tegmental area and mesencephalic raphe to the dorsal raphe nucleus in the rat. Evidence for a minor dopaminergic component. Exp Brain Res. 73, 69-77.

Kandel, E.R., Schwartz, J.H., Jessell, T.M., 2000. Principles of neural science. McGraw-Hill, New York, NY, pp. (4th ed.).

Khaldy, H., Leon, J., Escames, G., Bikjdaouene, L., Garcia, J.J., Acuna-Castroviejo, D., 2002. Circadian rhythms of dopamine and dihydroxyphenyl acetic acid in the mouse striatum: effects of pinealectomy and of melatonin treatment. Neuroendocrinology. 75, 201-208.

Kiehn, O., 2006. Locomotor circuits in the mammalian spinal cord. Annu Rev Neurosci. 29, 279-306. 
Kiehn, O., Butt, S.J., 2003. Physiological, anatomical and genetic identification of CPG neurons in the developing mammalian spinal cord. Prog Neurobiol. 70, 347-361.

Kiehn, O., Kjaerulff, O., 1996. Spatiotemporal characteristics of 5-HT and dopamine-induced rhythmic hindlimb activity in the in vitro neonatal rat. J Neurophysiol. 75, 1472-1482.

Kiehn, O., Kjaerulff, O., 1998. Distribution of central pattern generators for rhythmic motor outputs in the spinal cord of limbed vertebrates. Ann N Y Acad Sci. 860, 110-129.

Kjaerulff, O., Kiehn, O., 1996. Distribution of networks generating and coordinating locomotor activity in the neonatal rat spinal cord in vitro: a lesion study. J Neurosci. $16,5777-5794$.

Kjaerulff, O., Kiehn, O., 1997. Crossed rhythmic synaptic input to motoneurons during selective activation of the contralateral spinal locomotor network. J Neurosci. 17, 9433-9447.

Koeppen, A.H., 1995. The history of iron in the brain. J Neurol Sci. 134 Suppl, 1-9.

Koziol, B.J., Ohira, Y., Edgerton, V.R., Simpson, D.R., 1982. Changes in work tolerance associated with metabolic and physiological adjustment to moderate and severe iron deficiency anemia. Am J Clin Nutr. 36, 830-839.

Kremer, E., Lev-Tov, A., 1997. Localization of the spinal network associated with generation of hindlimb locomotion in the neonatal rat and organization of its transverse coupling system. J Neurophysiol. 77, 1155-1170.

Krishnan, P.R., Bhatia, M., Behari, M., 2003. Restless legs syndrome in Parkinson's disease: a case-controlled study. Mov Disord. 18, 181-185.

Kwik-Uribe, C.L., Gietzen, D., German, J.B., Golub, M.S., Keen, C.L., 2000a. Chronic marginal iron intakes during early development in mice result in persistent changes in dopamine metabolism and myelin composition. J Nutr. 130, 2821-2830.

Kwik-Uribe, C.L., Golub, M.S., Keen, C.L., 2000b. Chronic marginal iron intakes during early development in mice alter brain iron concentrations and behavior despite postnatal iron supplementation. J Nutr. 130, 2040-2048.

Kwik-Uribe, C.L., Golubt, M.S., Keen, C.L., 1999. Behavioral consequences of marginal iron deficiency during development in a murine model. Neurotoxicol Teratol. 21, 661-672.

Lafreniere-Roula, M., McCrea, D.A., 2005. Deletions of rhythmic motoneuron activity during fictive locomotion and scratch provide clues to the organization of the mammalian central pattern generator. J Neurophysiol. 94, 1120-1132.

Lai, Y.Y., Hsieh, K.C., Nguyen, D., Peever, J., Siegel, J.M., 2008. Neurotoxic lesions at the ventral mesopontine junction change sleep time and muscle activity during sleep: an animal model of motor disorders in sleep. Neuroscience. 154, 431-443.

Lapchak, P.A., Miller, P.J., Jiao, S., 1997. Glial cell line-derived neurotrophic factor induces the dopaminergic and cholinergic phenotype and increases locomotor activity in aged Fischer 344 rats. Neuroscience. 77, 745-752.

Lappe-Siefke, C., Goebbels, S., Gravel, M., Nicksch, E., Lee, J., Braun, P.E., Griffiths, I.R., Nave, K.A., 2003. Disruption of Cnp1 uncouples oligodendroglial functions in axonal support and myelination. Nat Genet. 33, 366-374.

Le Foll, B., Diaz, J., Sokoloff, P., 2005. Neuroadaptations to hyperdopaminergia in dopamine D3 receptor-deficient mice. Life Sci. 76, 1281-1296.

Lee, M.S., Choi, Y.C., Lee, S.H., Lee, S.B., 1996. Sleep-related periodic leg movements associated with spinal cord lesions. Mov Disord. 11, 719-722.

Levant, B., 1997. The D3 dopamine receptor: neurobiology and potential clinical relevance. Pharmacol Rev. 49, 231-252.

Levant, B., McCarson, K.E., 2001. D(3) dopamine receptors in rat spinal cord: implications for sensory and motor function. Neurosci Lett. 303, 9-12.

Levenson, C.W., Cutler, R.G., Ladenheim, B., Cadet, J.L., Hare, J., Mattson, M.P., 2004. Role of dietary iron restriction in a mouse model of Parkinson's disease. Exp Neurol. 190, 506-514. 
Li, W., Zhang, B., Tang, J., Cao, Q., Wu, Y., Wu, C., Guo, J., Ling, E.A., Liang, F., 2007. Sirtuin 2, a mammalian homolog of yeast silent information regulator-2 longevity regulator, is an oligodendroglial protein that decelerates cell differentiation through deacetylating alpha-tubulin. J Neurosci. 27, 2606-2616.

Li, X., Clark, J.D., 2001. Heme oxygenase inhibitors reduce formalin-induced Fos expression in mouse spinal cord tissue. Neuroscience. 105, 949-956.

Liebetanz, D., Baier, P.C., Paulus, W., Meuer, K., Bahr, M., Weishaupt, J.H., 2007. A highly sensitive automated complex running wheel test to detect latent motor deficits in the mouse MPTP model of Parkinson's disease. Exp Neurol. 205, 207-213.

Liebetanz, D., Merkler, D., 2006. Effects of commissural de- and remyelination on motor skill behaviour in the cuprizone mouse model of multiple sclerosis. Exp Neurol. 202, 217 224.

Lindvall, O., Bjorklund, A., Skagerberg, G., 1983. Dopamine-containing neurons in the spinal cord: anatomy and some functional aspects. Ann Neurol. 14, 255-260.

Liu, Q.S., Qiao, J.T., Dafny, N., 1992. D2 dopamine receptor involvement in spinal dopamineproduced antinociception. Life Sci. 51, 1485-1492.

Liu, X.G., Sandkuhler, J., 1995. Long-term potentiation of C-fiber-evoked potentials in the rat spinal dorsal horn is prevented by spinal N-methyl-D-aspartic acid receptor blockage. Neurosci Lett. 191, 43-46.

Loeffler, D.A., Connor, J.R., Juneau, P.L., Snyder, B.S., Kanaley, L., DeMaggio, A.J., Nguyen, H., Brickman, C.M., LeWitt, P.A., 1995. Transferrin and iron in normal, Alzheimer's disease, and Parkinson's disease brain regions. J Neurochem. 65, 710-724.

Lozoff, B., Beard, J., Connor, J., Barbara, F., Georgieff, M., Schallert, T., 2006. Long-lasting neural and behavioral effects of iron deficiency in infancy. Nutr Rev. 64, S34-43; discussion S72-91.

Lugaresi, E., Tassinari, C.A., Coccagna, G., 1965. Particularités cliniques et polygraphiques du syndrome d'impatience des membres inferieurs. Rev Neurol (Paris). 113, 545-552.

Luvisetto, S., Marinelli, S., Panasiti, M.S., D'Amato, F.R., Fletcher, C.F., Pavone, F., Pietrobon, D., 2006. Pain sensitivity in mice lacking the $\mathrm{Ca}(\mathrm{v}) 2.1$ alphal subunit of P/Q-type Ca2+ channels. Neuroscience. 142, 823-832.

Malmberg, A.B., Yaksh, T.L., 1992. Antinociceptive actions of spinal nonsteroidal antiinflammatory agents on the formalin test in the rat. J Pharmacol Exp Ther. 263, 136146.

Mansikka, H., Erbs, E., Borrelli, E., Pertovaara, A., 2005. Influence of the dopamine D2 receptor knockout on pain-related behavior in the mouse. Brain Res. 1052, 82-87.

McCrea, D.A., Pratt, C.A., Jordan, L.M., 1980. Renshaw cell activity and recurrent effects on motoneurons during fictive locomotion. J Neurophysiol. 44, 475-488.

Meiergerd, S.M., Patterson, T.A., Schenk, J.O., 1993. D2 receptors may modulate the function of the striatal transporter for dopamine: kinetic evidence from studies in vitro and in vivo. J Neurochem. 61, 764-767.

Mendell, L.M., 1966. Physiological properties of unmyelinated fiber projection to the spinal cord. Exp Neurol. 16, 316-332.

Michishita, E., Park, J.Y., Burneskis, J.M., Barrett, J.C., Horikawa, I., 2005. Evolutionarily conserved and nonconserved cellular localizations and functions of human SIRT proteins. Mol Biol Cell. 16, 4623-4635.

Millan, M.J., 2002. Descending control of pain. Prog Neurobiol. 66, 355-474.

Misgeld, T., Burgess, R.W., Lewis, R.M., Cunningham, J.M., Lichtman, J.W., Sanes, J.R., 2002. Roles of neurotransmitter in synapse formation: development of neuromuscular junctions lacking choline acetyltransferase. Neuron. 36, 635-648.

Mizuno, S., Mihara, T., Miyaoka, T., Inagaki, T., Horiguchi, J., 2005a. CSF iron, ferritin and transferrin levels in restless legs syndrome. J Sleep Res. 14, 43-47. 
Mizuno, S., Miyaoka, T., Inagaki, T., Horiguchi, J., 2005b. Prevalence of restless legs syndrome in non-institutionalized Japanese elderly. Psychiatry Clin Neurosci. 59, 461465.

Mizuo, K., Narita, M., Miyatake, M., Suzuki, T., 2004. Enhancement of dopamine-induced signaling responses in the forebrain of mice lacking dopamine D3 receptor. Neurosci Lett. 358, 13-16.

Moore, R.Y., 1996. Entrainment pathways and the functional organization of the circadian system. Prog Brain Res. 111, 103-119.

Morse, A.C., Beard, J.L., Jones, B.C., 1999. A genetic developmental model of iron deficiency: biological aspects. Proc Soc Exp Biol Med. 220, 147-152.

Murray, C.W., Porreca, F., Cowan, A., 1988. Methodological refinements to the mouse paw formalin test. An animal model of tonic pain. J Pharmacol Methods. 20, 175-186.

Murrin, L.C., Zeng, W.Y., 1990. Ontogeny of dopamine D1 receptors in rat forebrain: a quantitative autoradiographic study. Brain Res Dev Brain Res. 57, 7-13.

Myers, C.P., Lewcock, J.W., Hanson, M.G., Gosgnach, S., Aimone, J.B., Gage, F.H., Lee, K.F., Landmesser, L.T., Pfaff, S.L., 2005. Cholinergic input is required during embryonic development to mediate proper assembly of spinal locomotor circuits. Neuron. 46, 37-49.

Nave, K.A., Lai, C., Bloom, F.E., Milner, R.J., 1987. Splice site selection in the proteolipid protein (PLP) gene transcript and primary structure of the DM-20 protein of central nervous system myelin. Proc Natl Acad Sci U S A. 84, 5665-5669.

Nelson, C., Erikson, K., Pinero, D.J., Beard, J.L., 1997. In vivo dopamine metabolism is altered in iron-deficient anemic rats. J Nutr. 127, 2282-2288.

Nestler, E.J., Hyman, S.E., Malenka, R.C.,, 2001. Molecular Neuropharmacology “A Foundation For Clinical Neuroscience." McGraw-Hill, pp. 166-189, 255-261, 433-452.

Nishimaru, H., Takizawa, H., Kudo, N., 2000. 5-Hydroxytryptamine-induced locomotor rhythm in the neonatal mouse spinal cord in vitro. Neurosci Lett. 280, 187-190.

O'Keeffe, S.T., Gavin, K., Lavan, J.N., 1994. Iron status and restless legs syndrome in the elderly. Age Ageing. 23, 200-203.

O'Keeffe, S.T., Noel, J., Lavan, J.N., 1993. Restless legs syndrome in the elderly. Postgrad Med J. 69, 701-703.

Ohira, Y., Koziol, B.J., Edgerton, V.R., Brooks, G.A., 1981. Oxygen consumption and work capacity in iron-deficient anemic rats. J Nutr. 111, 17-25.

Oloyede, O.B., Folayan, A.T., Odutuga, A.A., 1992. Effects of low-iron status and deficiency of essential fatty acids on some biochemical constituents of rat brain. Biochem Int. 27, 913-922.

Ondo, W., Jankovic, J., 1996. Restless legs syndrome: clinicoetiologic correlates. Neurology. 47, 1435-1441.

Ondo, W.G., He, Y., Rajasekaran, S., Le, W.D., 2000. Clinical correlates of 6hydroxydopamine injections into A11 dopaminergic neurons in rats: a possible model for restless legs syndrome. Mov Disord. 15, 154-158.

Ondo, W.G., Vuong, K.D., Jankovic, J., 2002. Exploring the relationship between Parkinson disease and restless legs syndrome. Arch Neurol. 59, 421-424.

Ondo, W.G., Zhao, H.R., Le, W.D., 2007. Animal models of restless legs syndrome. Sleep Med. 8, 344-348.

Ortiz, E., Pasquini, J.M., Thompson, K., Felt, B., Butkus, G., Beard, J., Connor, J.R., 2004. Effect of manipulation of iron storage, transport, or availability on myelin composition and brain iron content in three different animal models. J Neurosci Res. 77, 681-689.

Outeiro, T.F., Kontopoulos, E., Altmann, S.M., Kufareva, I., Strathearn, K.E., Amore, A.M., Volk, C.B., Maxwell, M.M., Rochet, J.C., McLean, P.J., Young, A.B., Abagyan, R., Feany, M.B., Hyman, B.T., Kazantsev, A.G., 2007. Sirtuin 2 inhibitors rescue alphasynuclein-mediated toxicity in models of Parkinson's disease. Science. 317, 516-519. 
Pallos, J., Bodai, L., Lukacsovich, T., Purcell, J.M., Steffan, J.S., Thompson, L.M., Marsh, J.L., 2008. Inhibition of specific HDACs and sirtuins suppresses pathogenesis in a Drosophila model of Huntington's disease. Hum Mol Genet. 17, 3767-3775.

Parkes, J.D., Debono, A.G., Marsden, C.D., 1976. Bromocriptine in Parkinsonism: long-term treatment, dose response, and comparison with levodopa. J Neurol Neurosurg Psychiatry. 39, 1101-1108.

Paulus, W., Dowling, P., Rijsman, R., Stiasny-Kolster, K., Trenkwalder, C., 2007a. Update of the pathophysiology of the restless-legs-syndrome. Mov Disord. 22 Suppl 18, S431439.

Paulus, W., Dowling, P., Rijsman, R., Stiasny-Kolster, K., Trenkwalder, C., de Weerd, A., 2007b. Pathophysiological concepts of restless legs syndrome. Mov Disord. 22, 14511456.

Paulus, W., Trenkwalder, C., 2006. Less is more: pathophysiology of dopaminergic-therapyrelated augmentation in restless legs syndrome. Lancet Neurol. 5, 878-886.

Paxinos, G., Franklin, K.B.J., 2003. The Mouse Brain in Stereotaxic Coordinates. Academic Press, pp. 47.

Peterfreund, R.A., Kosofsky, B.E., Fink, J.S., 1995. Cellular localization of dopamine D2 receptor messenger RNA in the rat trigeminal ganglion. Anesth Analg. 81, 1181-1185.

Pichler, I., Hicks, A.A., Pramstaller, P.P., 2008. Restless legs syndrome: an update on genetics and future perspectives. Clin Genet. 73, 297-305.

Pinero, D.J., Li, N.Q., Connor, J.R., Beard, J.L., 2000. Variations in dietary iron alter brain iron metabolism in developing rats. J Nutr. 130, 254-263.

Ponting, C.P., 2001. Domain homologues of dopamine beta-hydroxylase and ferric reductase: roles for iron metabolism in neurodegenerative disorders? Hum Mol Genet. 10, 18531858.

Pratt, C.A., Jordan, L.M., 1987. Ia inhibitory interneurons and Renshaw cells as contributors to the spinal mechanisms of fictive locomotion. J Neurophysiol. 57, 56-71.

Presley, R.W., Menetrey, D., Levine, J.D., Basbaum, A.I., 1990. Systemic morphine suppresses noxious stimulus-evoked Fos protein-like immunoreactivity in the rat spinal cord. J Neurosci. 10, 323-335.

Qu, S., Le, W., Zhang, X., Xie, W., Zhang, A., Ondo, W.G., 2007. Locomotion is increased in a11-lesioned mice with iron deprivation: a possible animal model for restless legs syndrome. J Neuropathol Exp Neurol. 66, 383-388.

Qu, W.M., Huang, Z.L., Xu, X.H., Matsumoto, N., Urade, Y., 2008. Dopaminergic D1 and D2 receptors are essential for the arousal effect of modafinil. J Neurosci. 28, 8462-8469.

Rang, H.P., Dale, M.M., Ritter, J.M., , 1999. Pharmacology. Churchill Livingstone, pp. 94137, 579-595.

Reuss, S., 1996. Components and connections of the circadian timing system in mammals. Cell Tissue Res. 285, 353-378.

Rye, D.B., 2004. Parkinson's disease and RLS: the dopaminergic bridge. Sleep Med. 5, 317328.

Sachdev, P., 1995. The epidemiology of drug-induced akathisia: Part II. Chronic, tardive, and withdrawal akathisias. Schizophr Bull. 21, 451-461.

Salomon, J.A., Murray, C.J.L., 2000. The epidemiological transition revisited: new compositional models for mortality by age, sex and cause. Geneva, World Health Organization (GPE Discussion Paper No. 11, revised edition).

Salvi, F., Montagna, P., Plasmati, R., Rubboli, G., Cirignotta, F., Veilleux, M., Lugaresi, E., Tassinari, C.A., 1990. Restless legs syndrome and nocturnal myoclonus: initial clinical manifestation of familial amyloid polyneuropathy. J Neurol Neurosurg Psychiatry. 53, 522-525.

Sandkuhler, J., Liu, X., 1998. Induction of long-term potentiation at spinal synapses by noxious stimulation or nerve injury. Eur J Neurosci. 10, 2476-2480. 
Sanoja, R., Cervero, F., 2008. Estrogen modulation of ovariectomy-induced hyperalgesia in adult mice. Eur J Pain. 12, 573-581.

Sautel, F., Griffon, N., Sokoloff, P., Schwartz, J.C., Launay, C., Simon, P., Costentin, J., Schoenfelder, A., Garrido, F., Mann, A., et al., 1995. Nafadotride, a potent preferential dopamine D3 receptor antagonist, activates locomotion in rodents. J Pharmacol Exp Ther. 275, 1239-1246.

Schattschneider, J., Bode, A., Wasner, G., Binder, A., Deuschl, G., Baron, R., 2004. Idiopathic restless legs syndrome: abnormalities in central somatosensory processing. J Neurol. 251, 977-982.

Scheller, D., Ullmer, C., Berkels, R., Gwarek, M., Lubbert, H., 2009. The in vitro receptor profile of rotigotine: a new agent for the treatment of Parkinson's disease. Naunyn Schmiedebergs Arch Pharmacol. 379, 73-86.

Schmidt, B.J., Jordan, L.M., 2000. The role of serotonin in reflex modulation and locomotor rhythm production in the mammalian spinal cord. Brain Res Bull. 53, 689-710.

Schormair, B., Kemlink, D., Roeske, D., Eckstein, G., Xiong, L., Lichtner, P., Ripke, S., Trenkwalder, C., Zimprich, A., Stiasny-Kolster, K., Oertel, W., Bachmann, C.G., Paulus, W., Hogl, B., Frauscher, B., Gschliesser, V., Poewe, W., Peglau, I., Vodicka, P., Vavrova, J., Sonka, K., Nevsimalova, S., Montplaisir, J., Turecki, G., Rouleau, G., Gieger, C., Illig, T., Wichmann, H.E., Holsboer, F., Muller-Myhsok, B., Meitinger, T., Winkelmann, J., 2008. PTPRD (protein tyrosine phosphatase receptor type delta) is associated with restless legs syndrome. Nat Genet. 40, 946-948.

Shepherd, G.M., 1994. Neurobiology. Oxford University Press, pp. 267-284, 435-451.

Sherwin, C.M., 1998. Voluntary wheel running: a review and novel interpretation. Anim Behav. 56, 11-27.

Shiga, K., Hara, H., Okano, G., Aoyama, Y., 2003. Ingestion of water-soluble soybean fiber prevents gastrectomy-induced iron malabsorption, anemia and impairment of voluntary running exercise performance in rats. J Nutr. 133, 1120-1126.

Siegel, G.J., Agranoff, B.W., Albers, R.W., Fisher, S.K., Uhler, M.D., 1999. Basic Neurochemistry. Molecular, Cellular and Medical Aspects Sixth ed. Lippincott-Raven, Philadelphia,, pp. 637-689.

Skagerberg, G., Lindvall, O., 1985. Organization of diencephalic dopamine neurones projecting to the spinal cord in the rat. Brain Res. 342, 340-351.

Souayah, N., Sharovetskaya, A., Kurnellas, M.P., Myerson, M., Deitch, J.S., Elkabes, S., 2008. Reductions in motor unit number estimates (MUNE) precede motor neuron loss in the plasma membrane calcium ATPase 2 (PMCA2)-heterozygous mice. Exp Neurol. 214, 341-346.

Sowers, J.R., Vlachakis, N., 1984. Circadian variation in plasma dopamine levels in man. J Endocrinol Invest. 7, 341-345.

Stefansson, H., Rye, D.B., Hicks, A., Petursson, H., Ingason, A., Thorgeirsson, T.E., Palsson, S., Sigmundsson, T., Sigurdsson, A.P., Eiriksdottir, I., Soebech, E., Bliwise, D., Beck, J.M., Rosen, A., Waddy, S., Trotti, L.M., Iranzo, A., Thambisetty, M., Hardarson, G.A., Kristjansson, K., Gudmundsson, L.J., Thorsteinsdottir, U., Kong, A., Gulcher, J.R., Gudbjartsson, D., Stefansson, K., 2007. A genetic risk factor for periodic limb movements in sleep. N Engl J Med. 357, 639-647.

Stewart, C.C., 1898. Variations in daily activity produced by alcohol and by changes in barometric pressure and diet with a description of recording methods. American Journal of Physiology. 1, 40-56.

Stiasny-Kolster, K., Kohnen, R., Schollmayer, E., Moller, J.C., Oertel, W.H., 2004a. Patch application of the dopamine agonist rotigotine to patients with moderate to advanced stages of restless legs syndrome: a double-blind, placebo-controlled pilot study. Mov Disord. 19, 1432-1438. 
Stiasny-Kolster, K., Magerl, W., Oertel, W.H., Moller, J.C., Treede, R.D., 2004b. Static mechanical hyperalgesia without dynamic tactile allodynia in patients with restless legs syndrome. Brain. 127, 773-782.

Strong, P.N., Jr., 1957. Activity in the white rat as a function of apparatus and hunger. J Comp Physiol Psychol. 50, 596-600.

Sun, E.R., Chen, C.A., Ho, G., Earley, C.J., Allen, R.P., 1998. Iron and the restless legs syndrome. Sleep. 21, 371-377.

Suzuki, M., Hurd, Y.L., Sokoloff, P., Schwartz, J.C., Sedvall, G., 1998. D3 dopamine receptor mRNA is widely expressed in the human brain. Brain Res. 779, 58-74.

Svensson, K., Carlsson, A., Huff, R.M., Kling-Petersen, T., Waters, N., 1994. Behavioral and neurochemical data suggest functional differences between dopamine D2 and D3 receptors. Eur J Pharmacol. 263, 235-243.

Symonds, C.P., 1953. Nocturnal myoclonus. J Neurol Neurosurg Psychiatry. 16, 166-171.

Tan, E.K., Lum, S.Y., Wong, M.C., 2002. Restless legs syndrome in Parkinson's disease. J Neurol Sci. 196, 33-36.

Tan, E.K., Seah, A., See, S.J., Lim, E., Wong, M.C., Koh, K.K., 2001. Restless legs syndrome in an Asian population: A study in Singapore. Mov Disord. 16, 577-579.

Tang, L., Todd, R.D., O'Malley, K.L., 1994. Dopamine D2 and D3 receptors inhibit dopamine release. J Pharmacol Exp Ther. 270, 475-479.

Tao, Y.X., Johns, R.A., 2002. Activation and up-regulation of spinal cord nitric oxide receptor, soluble guanylate cyclase, after formalin injection into the rat hind paw. Neuroscience. 112, 439-446.

Tarazi, F.I., Baldessarini, R.J., 2000. Comparative postnatal development of dopamine D(1), $\mathrm{D}(2)$ and $\mathrm{D}(4)$ receptors in rat forebrain. Int J Dev Neurosci. 18, 29-37.

Tjolsen, A., Berge, O.G., Hunskaar, S., Rosland, J.H., Hole, K., 1992. The formalin test: an evaluation of the method. Pain. 51, 5-17.

Tomac, A., Lindqvist, E., Lin, L.F., Ogren, S.O., Young, D., Hoffer, B.J., Olson, L., 1995. Protection and repair of the nigrostriatal dopaminergic system by GDNF in vivo. Nature. 373, 335-339.

Treichler, F.R., Hall, J.F., 1962. The relationship between deprivation weight loss and several measures of activity. J Comp Physiol Psychol. 55, 346-349.

Trenkwalder, C., Paulus, W., Walters, A.S., 2005. The restless legs syndrome. Lancet Neurol. 4, 465-475.

Unger, E.L., Earley, C.J., Beard, J.L., 2008. Diurnal cycle influences peripheral and brain iron levels in mice. J Appl Physiol.

Walters, A.S., 1995. Toward a better definition of the restless legs syndrome. The International Restless Legs Syndrome Study Group. Mov Disord. 10, 634-642.

Walters, A.S., Hening, W., Rubinstein, M., Chokroverty, S., 1991. A clinical and polysomnographic comparison of neuroleptic-induced akathisia and the idiopathic restless legs syndrome. Sleep. 14, 339-345.

Walters, A.S., Ondo, W.G., Zhu, W., Le, W., 2009. Does the endogenous opiate system play a role in the Restless Legs Syndrome?: A pilot post-mortem study. J Neurol Sci.

Wang, J., O'Reilly, B., Venkataraman, R., Mysliwiec, V., Mysliwiec, A., 2009. Efficacy of oral iron in patients with restless legs syndrome and a low-normal ferritin: A randomized, double-blind, placebo-controlled study. Sleep Med.

Ward, K.L., Tkac, I., Jing, Y., Felt, B., Beard, J., Connor, J., Schallert, T., Georgieff, M.K., Rao, R., 2007. Gestational and lactational iron deficiency alters the developing striatal metabolome and associated behaviors in young rats. J Nutr. 137, 1043-1049.

Weber, M., Lauterburg, T., Tobler, I., Burgunder, J.M., 2004. Circadian patterns of neurotransmitter related gene expression in motor regions of the rat brain. Neurosci Lett. 358, 17-20.

Werner, H.B., Kuhlmann, K., Shen, S., Uecker, M., Schardt, A., Dimova, K., Orfaniotou, F., Dhaunchak, A., Brinkmann, B.G., Mobius, W., Guarente, L., Casaccia-Bonnefil, P., 
Jahn, O., Nave, K.A., 2007. Proteolipid protein is required for transport of sirtuin 2 into CNS myelin. J Neurosci. 27, 7717-7730.

Willis, T., 1685. The London practice of physick. London: Bassett and Crooke, pp.

Wilson, D.M., 1961. The central nervous control of flight in a locust. J. Exp. Biol. 38, 471490.

Winkelman, J.W., 1999. The evoked heart rate response to periodic leg movements of sleep. Sleep. 22, 575-580.

Winkelmann, J., Schormair, B., Lichtner, P., Ripke, S., Xiong, L., Jalilzadeh, S., Fulda, S., Putz, B., Eckstein, G., Hauk, S., Trenkwalder, C., Zimprich, A., Stiasny-Kolster, K., Oertel, W., Bachmann, C.G., Paulus, W., Peglau, I., Eisensehr, I., Montplaisir, J., Turecki, G., Rouleau, G., Gieger, C., Illig, T., Wichmann, H.E., Holsboer, F., MullerMyhsok, B., Meitinger, T., 2007. Genome-wide association study of restless legs syndrome identifies common variants in three genomic regions. Nat Genet. 39, 10001006.

Wong, J.Y., Clifford, J.J., Massalas, J.S., Finkelstein, D.I., Horne, M.K., Waddington, J.L., Drago, J., 2003. Neurochemical changes in dopamine D1, D3 and D1/D3 receptor knockout mice. Eur J Pharmacol. 472, 39-47.

Wu, D., Hersh, L.B., 1994. Choline acetyltransferase: celebrating its fiftieth year. J Neurochem. 62, 1653-1663.

Xie, G.X., Jones, K., Peroutka, S.J., Palmer, P.P., 1998. Detection of mRNAs and alternatively spliced transcripts of dopamine receptors in rat peripheral sensory and sympathetic ganglia. Brain Res. 785, 129-135.

Xu, M., Koeltzow, T.E., Santiago, G.T., Moratalla, R., Cooper, D.C., Hu, X.T., White, N.M., Graybiel, A.M., White, F.J., Tonegawa, S., 1997. Dopamine D3 receptor mutant mice exhibit increased behavioral sensitivity to concurrent stimulation of D1 and D2 receptors. Neuron. 19, 837-848.

Yaksh, T.L., Hua, X.Y., Kalcheva, I., Nozaki-Taguchi, N., Marsala, M., 1999. The spinal biology in humans and animals of pain states generated by persistent small afferent input. Proc Natl Acad Sci U S A. 96, 7680-7686.

Yamamoto, H., Schoonjans, K., Auwerx, J., 2007. Sirtuin functions in health and disease. Mol Endocrinol. 21, 1745-1755.

Yamamoto, T., Yaksh, T.L., 1992. Comparison of the antinociceptive effects of pre- and posttreatment with intrathecal morphine and MK801, an NMDA antagonist, on the formalin test in the rat. Anesthesiology. 77, 757-763.

Yang, H.W., Zhou, L.J., Hu, N.W., Xin, W.J., Liu, X.G., 2005. Activation of spinal d1/d5 receptors induces late-phase LTP of C-fiber-evoked field potentials in rat spinal dorsal horn. J Neurophysiol. 94, 961-967.

Yehuda, S., Youdim, M.B., 1984. The increased opiate action of beta-endorphin in irondeficient rats: the possible involvement of dopamine. Eur J Pharmacol. 104, 245-251.

Yehuda, S., Youdim, M.B., 1989. Brain iron: a lesson from animal models. Am J Clin Nutr. 50, 618-625; discussion 625-619.

Yehuda, S., Youdim, M.E., Mostofsky, D.I., 1986. Brain iron-deficiency causes reduced learning capacity in rats. Pharmacol Biochem Behav. 25, 141-144.

Yehuda, S., Youdim, M.B.H. , 1988. Brain iron deficiency: biochemistry and behaviour, in: M.B.H. Youdim Ed. , Brain Iron: Neurochemical and Behavioural Aspects,. Taylor and Francis, London, pp. 89-114.

Youdim, M.B., Ashkenazi, R., Ben-Shachar, D., Yehuda, S., 1984. Modulation of dopamine receptor in the striatum by iron: behavioral and biochemical correlates. Adv Neurol. 40, 159-170.

Youdim, M.B., Ben-Shachar, D., Ashkenazi, R., Yehuda, S., 1983. Brain iron and dopamine receptor function. Adv Biochem Psychopharmacol. 37, 309-321.

Youdim, M.B., Ben-Shachar, D., Riederer, P., 1993. The possible role of iron in the etiopathology of Parkinson's disease. Mov Disord. 8, 1-12. 
Youdim, M.B., Green, A.R., 1976. Biogenic monoamine metabolism and functional activity in iron-deficient rats: behavioural correlates. Ciba Found Symp. 201-225.

Youdim, M.B., Green, A.R., 1978. Iron deficiency and neurotransmitter synthesis and function. Proc Nutr Soc. 37, 173-179.

Youdim, M.B., Sills, M.A., Heydorn, W.E., Creed, G.J., Jacobowitz, D.M., 1986. Iron deficiency alters discrete proteins in rat caudate nucleus and nucleus accumbens. $\mathrm{J}$ Neurochem. 47, 794-799.

Youdim, M.B., Yehuda, S., Ben-Uriah, Y., 1981. Iron deficiency-induced circadian rhythm reversal of dopaminergic-mediated behaviours and thermoregulation in rats. Eur $\mathbf{J}$ Pharmacol. 74, 295-301.

Yu, G.S., Steinkirchner, T.M., Rao, G.A., Larkin, E.C., 1986. Effect of prenatal iron deficiency on myelination in rat pups. Am J Pathol. 125, 620-624.

Zarrindast, M.R., Moghaddampour, E., 1989. Opposing influences of D-1 and D-2 dopamine receptors activation on morphine-induced antinociception. Arch Int Pharmacodyn Ther. 300, 37-50.

Zarrindast, M.R., Nassiri-Rad, S., Pazouki, M., 1999. Effects of dopaminergic agents on antinociception in formalin test. Gen Pharmacol. 32, 517-522.

Zehr, E.P., Carroll, T.J., Chua, R., Collins, D.F., Frigon, A., Haridas, C., Hundza, S.R., Thompson, A.K., 2004. Possible contributions of CPG activity to the control of rhythmic human arm movement. Can J Physiol Pharmacol. 82, 556-568.

Zhang, Y., Narayan, S., Geiman, E., Lanuza, G.M., Velasquez, T., Shanks, B., Akay, T., Dyck, J., Pearson, K., Gosgnach, S., Fan, C.M., Goulding, M., 2008. V3 spinal neurons establish a robust and balanced locomotor rhythm during walking. Neuron. 60, 84-96.

Zhao, H., Zhu, W., Pan, T., Xie, W., Zhang, A., Ondo, W.G., Le, W., 2007. Spinal cord dopamine receptor expression and function in mice with 6-OHDA lesion of the A11 nucleus and dietary iron deprivation. J Neurosci Res. 85, 1065-1076.

Zhu, H., Clemens, S., Sawchuk, M., Hochman, S., 2007. Expression and distribution of all dopamine receptor subtypes $(\mathrm{D}(1)-\mathrm{D}(5))$ in the mouse lumbar spinal cord: a real-time polymerase chain reaction and non-autoradiographic in situ hybridization study. Neuroscience. 149, 885-897. 


\title{
Curriculum vitae
}

\author{
Dr. Pascal Dowling, BScPharm (Hons), MPhil, PhD \\ Pharmacologist
}

Email: pdowling@med.uni-goettingen.de,phd.pharma@web.de

Profile

Qualities

Education
A determined and resourceful postgraduate with collaborative and communicative skills. I describe myself as being very capable and a fast learner. Born on April $10^{\text {th }} 1983$, a British national.

Excellent organisational skills and a strong approach to problem solving. Working to deadlines, performing well under pressure and working efficiently in a team environment.

All early education was completed in England. 1999-2001. Truro College, Cornwall, UK.

Obtained A-Levels in Chemistry, Mathematics and Physics.

2001-2004. University of Manchester, Dept. of Biological Sciences, Manchester, UK.

Obtained my first degree B.Sc(Hons) in Pharmacology. This three year course involved studying many topics. Example of topics covered; Drug Discovery, Drug Disposition, Neuropharmacology, Advanced Neuropharmacology, Bioinformatics, Endocrinology Biostatistics, Brain Structure and function, Drug Design, Ion Channels and Enzymes as Drug Targets, Pharmaceutical Chemistry, and Molecular Toxicology. Acquired a strong experience with a variety of techniques ranging from ELISA to gene cloning and rodent animal behavioural studies. Received insight into the science of high throughput screening and development of novel compounds from GSK and AstraZeneca led discussions.

The final year project of my degree was titled "Interaction of epibatidine with the resting state of the foetal muscle nicotinic acetylcholine receptor." Employed radioligand binding assays and electrophysiological (patch clamp) studies to show the importance of epibatidine selectivity upon certain residues on $y$ and $\delta$ subunits of the muscle nAChRs when in the resting conformation state. Other techniques were used including sitedirected mutagenesis, restriction digest of DNA, transformation of DH5a bacteria, transfection of mutant DNA for patch clamp and binding assays, culturing of bacteria and 293 HEK cells.

2004-2005. Cardiff University, Dept. of Biological Sciences, Cardiff, UK. Received a Master in Philosophy (M.Phil) within the field of Neuroscience, supervised by Dr. Robert Santer. This course was a purely research based Masters, where a 150 page classical thesis was produced and examined along with other relevant material (publications) by a 3-4 hour viva. The title of my Masters thesis was "Age Related Changes in Preganglionic and Postganglionic Neurons in the Major Pelvic Ganglion of the Male Rat." Techniques covered were transmission electron microscopy (TEM), general histochemistry procedures and fluorescence microscopy. 
2006. Max Planck Institute for Biophysical Chemistry (Karl Friedrich Bonhoeffer Institute), Dept. of Molecular Cell Biology, Tower 5, Am Faßberg 11, 37077 Göttingen, Germany.

Completed 5 months of research (March 2006-August 2006) working under the supervision of Professor. Dr. Ahmed Mansouri. My topic was to investigate a gene which is an important dopamine neuron determinant. Precultured embryonic stem cells were selected by FACS according to a transcription factor of this gene. The result would be a purified stem cell population prior to implanting into the depleted ventral midbrain region of a rat model of Parkinson's disease.

Learned many skills including culturing, harvesting and electroporation of mouse and spermatogenic human embryonic stem cells. In addition, other relevant cloning skills were learnt, these included checking homogeneity with other related gene members, designing primers and ligation into pGEM TEasy vector, verified by gel electrophoresis and sequencing. Moreover, the vector was cultured in E.coli LB and plated followed by purification of DNA. Moreover, PCR was also performed. Finally labelling of the probe and determination of the DNA fragment, the latter was performed by Southern blot analysis.

2006-2009. Georg-August University School of Science (GAUSS) PhD program, Elite University of Göttingen, Germany.

University Medical Centre Göttingen (UMG), Georg-August University, Dept. of Clinical Neurophysiology, Robert-Koch-Straße, 37075 Göttingen, Germany.

Completed a Doctorate in Philosophy (Ph.D.) in the field of Neuroscience. Received a stipend from both the Deutsche Forschungsgemeinschaft (DFG) funded European Graduate College (GK 632) and the Department of Clinical Neurophysiology. Officially my PhD started in August 2006 and finished in March 2009. My supervisors were Professor. Dr. med. Walter Paulus and Dr. David Liebetanz. The title of my thesis was: "Impact of iron-deficiency upon behaviour and protein expression in the male mouse with implications for Restless Legs Syndrome". Animal behavioural experiments were implemented and an animal model was established. Surgical techniques were used, including blood/tissue extraction, preparation and analysis. Immunohistochemistry, both peroxidase and fluorescence techniques. Molecular techniques were performed; these were 1-D/2-D DIGE electrophoresis, MALDI-TOF-MS and Western Blotting both TANK and Semi-Dry. A classical thesis was written and examined by a presentation in addition to an intellectual Rigour (Rigorosum) on the topics, intracellular signaling mechanisms and central nervous regulation of sleep and pain. In addition, teaching, conference and presentation credits were completed.

PhD

Collaborators
2007-2009

Max Planck Institute for Experimental Medicine, Hermann-Rein-Straße 3, 37075 Göttingen, Germany.

Dr. Hauke Werner, Department of Neurogenetics

Dr. Olaf Jahn, Proteomics Group

Centre for Clinical Research, 89081 UIm, Germany. Professor Dr. Markus Otto, Neurology Group.

University Medical Centre, Göttingen, Germany.

Professor Dr. med. Christine Stadelmann-Nessler, Neuropathology.

UCB Pharma S.A., Belgium, Brussels.

Acquired Rotigotine from UCB Pharma. Professor. Dr. Dieter Scheller, Director of Pharmacology in Parkinson's Disease and Movement Disorders. 
Work

Experience

Training

Courses

2001-2009

2002-2004

ICON Development Solutions, Clinical Research Laboratory, Skelton

House, Manchester Science Park, Manchester, UK.

Formerly known as Medeval, a phase 1 contract research organisation (CRO). During my pharmacology degree I worked part-time as a Locum lab and clinical analyst. Obtained a solid foundation of team work in a professional environment concerned directly with clinical trials of potential novel market drugs.

\section{3-2004}

University of Manchester.

Completed an in-vivo pharmacology course supported by the British Pharmacological Society and organised by the British Home Office. This course was examined by the University of Manchester and titled "In Vivo Pharmacology Practical Unit BS308." The course involved learning surgical procedures, anaesthetics, animal handling/behavioural experiments (rats and mice only), bioethics and legal knowledge.

\section{5}

Cardiff University, Biomedical Sciences Building.

Performed a 3 month course at the Biosciences Electron Microscopy Unit, supervised by Dr. Anthony Hann.

\section{6}

Georg-August University, 37075 Göttingen, Germany.

Participated in a "Rhetoric and Presentation Course" organised by the European DFG Graduiertenkolleg GK 632.

2006

Max Planck Institute for Biophysical Chemistry (Karl Friedrich Bonhoeffer Institute), Am Faßberg 11, 37077 Göttingen, Germany.

Attended a two-day advanced methods course, whereby my topic of choice was electrophysiology and patch clamp recordings at the laboratory of Professor Erwin Neher.

\section{7/2008}

Centre for Clinical Research, 89081 UIm, Germany.

Attended a 10 day proteomics course, where 2-D DIGE was learnt and 2-D IEF/SDS-PAGE. Protein levels prior to gel-run were pre-determined by BCA assay.

\section{8}

Max Planck Institute for Experimental Medicine, Proteomics Group, Hermann-Rein-Straße 3, 37075 Göttingen, Germany.

Attended a 7 day proteomics course. Observed the techniques involved for spotting onto the MALDI target in addition to MALDI-TOF-MS. I also learnt and performed TANK Western blotting and related procedures.

\section{1-2009}

I have had numerous computer training courses over my years of higher education which has made me feel confident with using computers to a good level, including all Microsoft Office tools for Windows and Mac. Furthermore, I am proficient in software concerning data entry, this includes Minitab 14, Prism and SPSS. Currently learning MATLAB. 
Dowling, P., Ranson, R.N., Santer, R.M., 2006. Age-associated changes in distribution of the $\mathrm{P} 2 \mathrm{X}_{2}$ receptor in the major pelvic ganglion of the male rat. Neuroscience Letters. 404(3), 320-323.

Ranson, R.N., Dowling, P., Santer, R.M., Watson, A.H., 2007. The effects of ageing on the distribution of vesicular acetylcholine transporter immunoreactive inputs to pelvic motoneurons of male Wistar rats. Neuroscience. 144(2), 636-644.

Paulus, W., Dowling, P., Rijsman, R., Stiasny-Kolster, K., Trenkwalder, C., de Weerd, A., 2007. Pathophysiological concepts of RLS. Movement Disorders. 22(10), 1451-1456.

Paulus, W., Dowling, P., Rijsman, R., Stiasny-Kolster, K., Trenkwalder, C., 2007. Update of the pathophysiology of the Restless-Legs-Syndrome. Movement Disorders. 22(S18), S431-S439.

Dowling, P., Klinker, F., Amaya, F., Paulus, W., Liebetanz, D., (2009). Sensitisation of iron-deficient mice to acute pain stimuli and formalin-induced nociception. Journal of Nutrition. Accepted.

Dowling, P., Klinker, F., Stadelmann, C., Hasan, K., Paulus, W., Liebetanz, D., (2009). D3 receptors maintain altered sensorimotor behaviour induced by iron-deficiency. In Preparation

Dowling, P., Lehnert, S., Jahn, O., Otto, M., Paulus, W., Liebetanz, D., (2009). 2-D Gel-electrophoresis and MALDI-TOF identify novel proteins dysregulated in the midbrain of iron-deficient mice. In Preparation.

Dowling, P., Stadelmann, C., Paulus, W., Liebetanz, D., (2009). Investigating the diencephalic A11 region in iron-deficient mice. In Preparation

Klinker, F., Hasan, K., Dowling, P., Paulus, W., Liebetanz, D., (2009). Increased impact of iron-deficiency on motor learning in D3R-KO mice. In Preparation.

Dowling, P., Ranson, R.N., Santer, R.M., Watson, A.H., (2009). Ageassociated changes in distribution of methionine ${ }^{5}$-enkephalin and vesicular acetylcholine transporter in the major pelvic ganglion of the male rat. In Preparation.

\section{Scientific Abstracts}

Dowling, P., Ranson, R.N., Santer, R.M. Vesicular acetylcholine transporter- and met-enkephalin-containing terminals on neurones of the major pelvic ganglion in young and aged rats. Journal of Anatomy. 208, 395413, 2006.

Dowling, P., Paulus, W., Liebetanz, D., 2008. Elevated acute and chronic pain responses in iron-deficient mice. FENS Abstr. vol 4, 189.8, 2008 
Spoken

Presentation

Skills

Prizes

Poster

Presentation

Skills

Congresses

Teaching

Languages

Extra-

Curricular

Activities
2000 (Title: "Venlafaxine and its use as an atypical antidepressant").

2002 (Title: "Pharmacokinetics of the nicotine patch").

2003 (Title: "Phosphodiesterase inhibitor sildenafil").

2006 (Title: "D2 receptor regulates the development of dopaminergic neurons via extracellular signal-regulated kinase and Nurr1 activation").

2007 (Title: "Animal behavioural alterations during a state of induced iron-deficiency").

2008 (Title: "Protein expression differences during iron-deficiency").

2009 (Title: "Impact of iron-deficiency upon behaviour and protein expression in the male mouse with implications for Restless Legs Syndrome").

2009 (Title: "Central nervous regulation of the motor system").

2009. Best Project Prize. Bayer Vital sponsored department retreat.

Anatomical Society of Great Britain \& Ireland Summer Meeting 2005.

Cardiff University. Cardiff, UK.

German Neuroscience Society (GNS) Summer Meeting 2007. Georg-August University. Göttingen, Germany.

Federation of European Neuroscience Societies (FENS) Summer Meeting 2008. Geneva, Switzerland.

Anatomical Society of Great Britain \& Ireland Summer Meeting 2005.

Cardiff University. Cardiff, UK.

Federation of European Neuroscience Societies (FENS) Summer Meeting 2006. Vienna, Austria.

Federation of European Neuroscience Societies (FENS) Summer Meeting 2008. Geneva, Switzerland.

European Restless Legs Syndrome Study Group (EURLSSG) Winter Meeting. Munich, Germany.

Headed a tutorial on Central Motor Systems for students in the MSc/PhD Neuroscience Program (Georg-August University Göttingen / International Max Planck Research School).

Taught many students animal behavioural methods and basic laboratory techniques during my $\mathrm{PhD}$.

English: Mother's tongue

German: Fair reading, writing and conversational skills

French: Basic level

Indoors: Swimming, squash, Taekwon-Do.

Outdoors: Tennis, skiing. Earlier played many sports, including cricket and rugby for both county and West of England. 